\title{
INVESTIGATION OF HYBRID SAGD USING CARBON DIOXIDE, PROPANE, NITROGEN AND METHANE
}

\author{
by
}

\section{Muhammad Imran}

B.Sc. in Chemical Engineering, University of Engineering and Technology, Pakistan, 1996 M.A.Sc. in Chemical Engineering, Ryerson University, Toronto, 2008

A dissertation presented to Ryerson University

\author{
in partial fulfillment of the \\ requirements for the degree of \\ Doctor of Philosophy \\ in the program of

\section{Chemical Engineering}

Toronto, Ontario, Canada, 2018

CMuhammad Imran, 2018 


\section{Author's Declaration}

I hereby declare that I am the sole author of this dissertation. This is a true copy of the dissertation, including any required final revisions, as accepted by my examiners.

I authorize Ryerson University to lend this dissertation to other institutions or individuals for the purpose of scholarly research.

I further authorize Ryerson University to reproduce this dissertation by photocopying or by other means, in total or in part, at the request of other institutions or individuals for the purpose of scholarly research.

I understand that my dissertation may be made electronically available to the public. 


\title{
INVESTIGATION OF HYBRID SAGD USING CARBON DIOXIDE, PROPANE, NITROGEN AND METHANE
}

\author{
Doctor of Philosophy, 2018 \\ Muhammad Imran \\ Chemical Engineering \\ Ryerson University
}

\begin{abstract}
This study investigates a hybrid SAGD (steam-assisted gravity drainage) process utilizing four gaseous solvents, namely, carbon dioxide, propane, nitrogen and methane that are co-injected with steam at different concentrations of 5.0, 7.5 and $10.0 \mathrm{wt} \%$. The objectives are to evaluate and compare the effectiveness of non-condensable gases like methane, nitrogen and carbon dioxide with those of condensable hydrocarbons like propane; to evaluate the performance of hybrid SAGD applied to depleted, low-pressure oil reservoirs; and to numerically simulate the experimental results and obtain tuned relative permeability curves. For this purpose, rigorous experimentation is done using a laboratory-scale, cylindrical replica (i.e., physical model) of an oil reservoir with a set of parallel horizontal injection and production wells. A numerical process model is developed, simulated, and calibrated with the help of experimental data.
\end{abstract}

The experimental setup incorporates i) an injection system designed to co-inject solvent and steam at the required injection temperature of $195^{\circ} \mathrm{C}$ and pressure of $1.45 \mathrm{MPa}$, gauge; ii) a production system designed to collect the produced fluids and measure the fractional flow of each phase while ensuring smooth operation with minimal variations in production pressure; and iii) control systems 
designed to precisely control the heaters temperatures. The experiments are performed at isothermal conditions with model permeability and porosity, respectively, 10.7 Darcy and 32\%.

It is observed that for low pressure reservoirs, oil recoveries with co-injected solvents are at least $18 \%$ more than that from steam alone. On an equal-weight-percentage basis, methane is found to be the best solvent, and results in the highest oil recovery of $50.7 \%$ of the original oil in place. Compared to non-condensable gases, propane has the highest solvent retention of up to $15 \%$. The gases with higher solubility in heavy oil, like carbon dioxide and propane, show a reduction in oil recovery with an increase in feed solvent concentration.

A numerical model of the process is developed and simulated using Computer Modelling Group's (CMG) WinProp and STARS simulators. For the solvents that are found to be promising, the simulated oil, water and gas recoveries are history-matched with their experimental counterparts by adjusting the relative permeability curves. The resulting, calibrated model is able to predict oil, water and gas recovery in the hybrid SAGD process with less than 5\% relative error. 


\section{Acknowledgments}

I highly appreciate the help and support from my supervisors, Professors Simant R. Upreti, Ali Lohi, and Farhad Ein-Mozaffari.

My special thanks go to my supervisor at Saskatchewan Research Council, Kelvin D. Knorr, who has always supported me in completing my PhD study. His mentorship and guidance immensely contributed to the fulfilment of this dissertation.

I would also like to acknowledge the Saskatchewan Research Council for its financial support for building of the experimental setup, and for permission to carry out the experiments.

Finally, this thesis is dedicated to my parents, wife and children, whose continuous backing and encouragement made this possible. 


\section{Table of Contents}

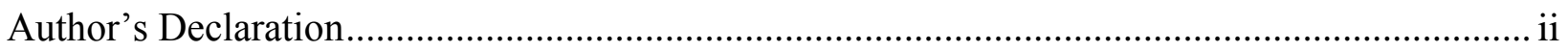

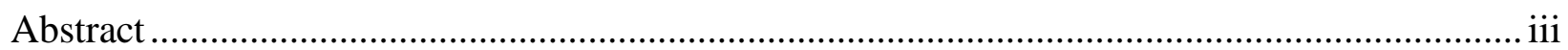

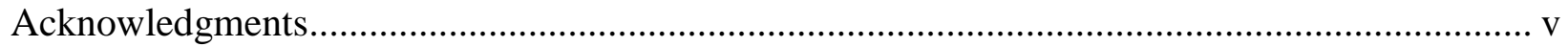

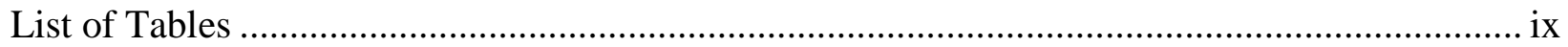

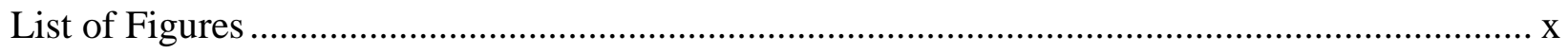

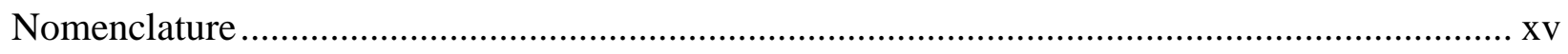

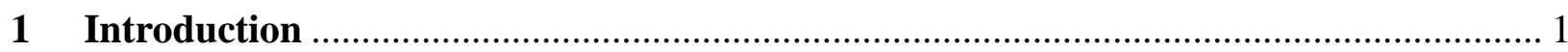

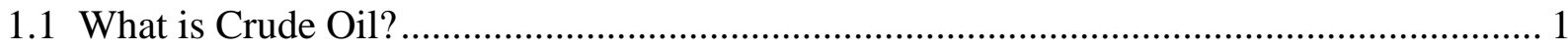

1.2 Natural Bitumen and Extra Heavy Oil ...................................................................... 2

1.3 Cold Heavy Oil and Bitumen Recovery Technologies ......................................................... 5

1.3.1 Cold Heavy Oil Production with Sands (CHOPS) …………........................................ 5

1.3.2 Heavy Oil Waterflooding.............................................................................. 7

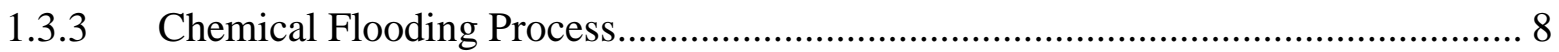

1.3.4 Vapour Extraction of Heavy Oil (Vapex) .......................................................... 10

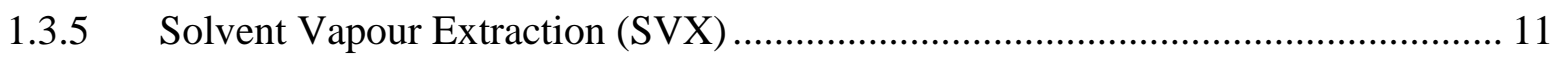

1.3.6 DME Injection Process ................................................................................... 12

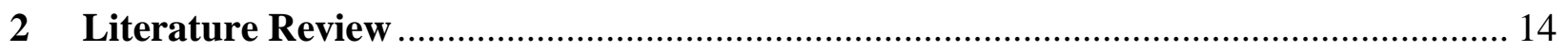

2.1 Thermal Heavy Oil and Bitumen Recovery Technologies .................................................. 14

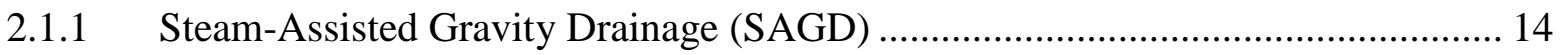

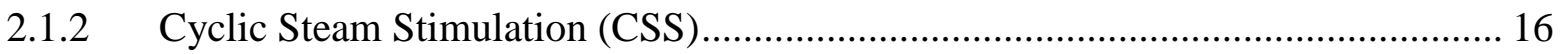

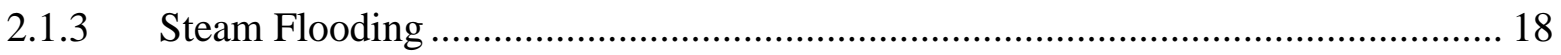

2.1.4 Electrical Heating Processes ………………........................................................ 19

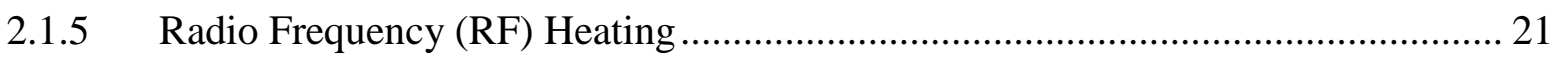




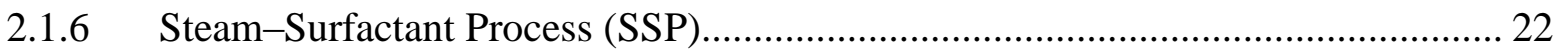

2.2 Physical and Numerical Modelling of Hybrid SAGD Processes .................................... 24

2.2.1 Solvent-Aided Process (SAP) ............................................................................... 25

2.2.2 Expanding Solvent SAGD (ES-SAGD) …………............................................... 29

2.2.3 SAGD with Non-Condensable Gases................................................................... 37

2.2.4 Hybrid SAGD - Some Field Examples ......................................................................... 39

2.3 Scope for Further Development of Hybrid SAGD......................................................... 40

2.4 Objectives of Current Work ……………………………............................................... 42

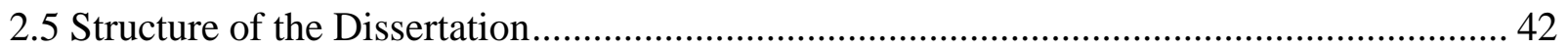

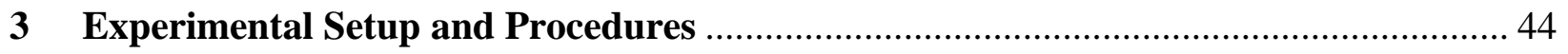

3.1 Physical Model Experimental Apparatus ........................................................................... 44

3.2 Synthetic Matrix Preparation and Model Packing .............................................................. 48

3.3 Synthetic Porous Matrix and Fluid Properties .................................................................... 49

3.4 Experimental Procedure ………………………………............................................. 55

3.5 Data Accuracy and Quality Check …………………………........................................ 59

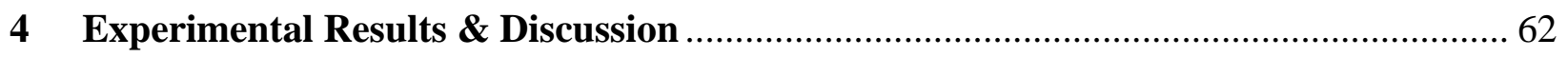

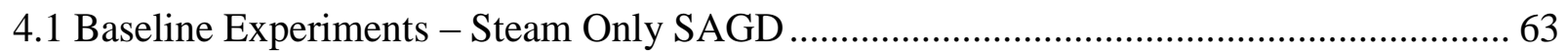

4.2 Steam Plus Carbon Dioxide Injection Experiments ............................................................. 66

4.3 Steam Plus Propane Injection Experiments ..................................................................... 74

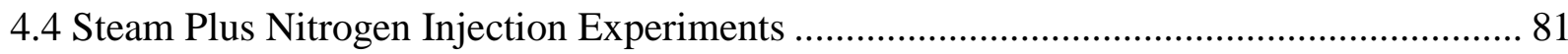

4.5 Steam Plus Methane Injection Experiments................................................................... 88

4.6 Effect of Solvent Type on Hybrid SAGD Performance .......................................................... 95

4.7 Important Observations and Findings from Experimental Study.................................... 102

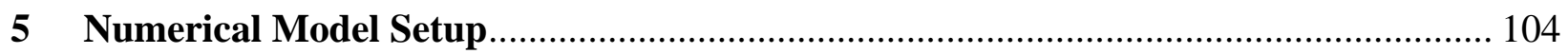


5.1 Equation of State (EOS) Fluid Model ................................................................. 104

5.2 Reservoir Model ........................................................................................... 112

6 Simulation Results and Discussion................................................................... 122

6.1 History-Match Results for Steam plus Carbon Dioxide Experiments .......................... 123

6.2 History-Match Results for Steam plus Nitrogen Experiments.................................. 132

6.3 Hisory-Match Results for Steam plus Methane Experiments .................................... 140

6.4 Important Observations and Findings from Simulation Study .................................. 147

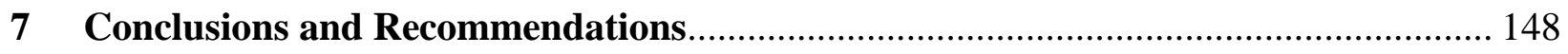

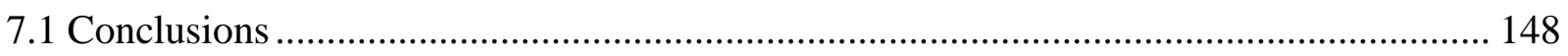

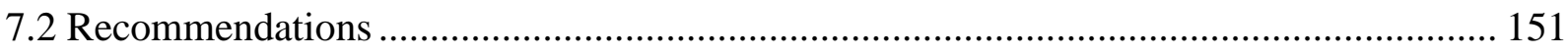

Appendix A: Model Temperature Plots ..................................................................... 152

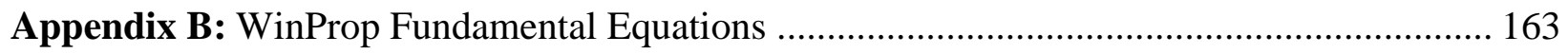

Appendix C: K-Value, Density and Viscosity Data for Fluid Model Tuning ......................... 165

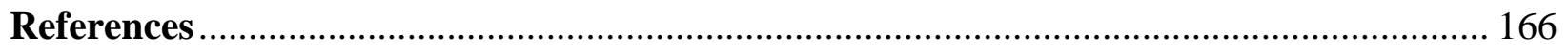




\section{List of Tables}

Table 2.1 Simulation model reservoir properties (Gupta et al., 2010) ..................................... 27

Table 2.2 Butane solvent injection strategies (Gupta et al., 2010) ......................................... 27

Table 2.3 Geological model properties (Akinboyewa et al., 2010) ........................................ 28

Table 2.4 Model dimensions and properties (Khaledi et al., 2012) ........................................ 30

Table 2.5 Model dimensions and properties (Jiang et al., 2012) ............................................. 32

Table 2.6 Numerical simulator input parameters (Gates and Chakrabarty, 2008) .................... 34

Table 2.7 3D physical model dimensions (Ivory et al., 2007) .............................................. 36

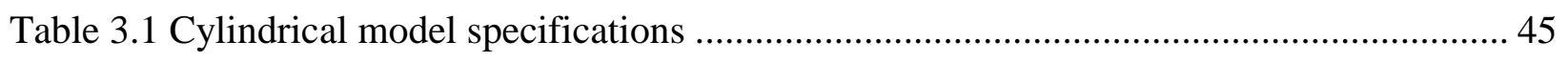

Table 3.2 Model packing saturations and volumes.......................................................... 49

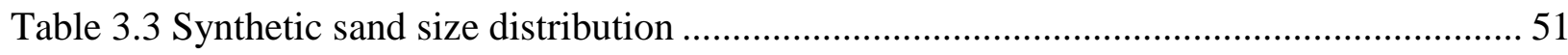

Table 3.4 Synthetic sand permeability at different flow rates .............................................. 52

Table 3.5 Oil density at different temperatures and atmospheric pressure ............................... 54

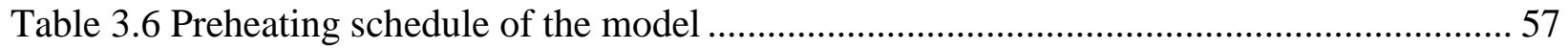

Table 3.7 Gaseous solvent injection rates with CWE steam rate of $115 \mathrm{~mL} / \mathrm{min}$................... 58

Table 3.8 Pressure transducer and differential pressure accuracy ........................................ 60

Table $5.1 \mathrm{~K}$-values for carbon dioxide-oil ................................................................. 111

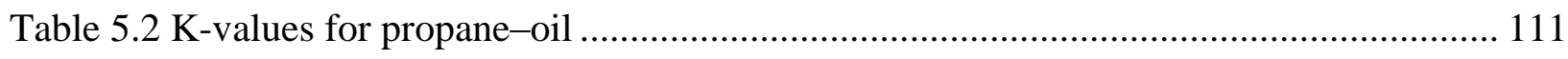

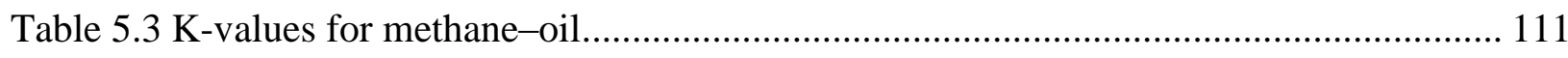

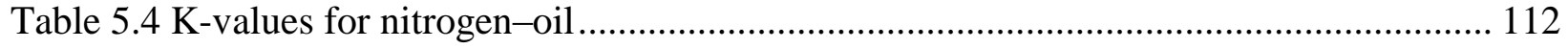

Table 6.1 History matching parameters …................................................................ 124 


\section{List of Figures}

Figure 1.1 World oil reserves by region (from US Energy Information Administration, 2007).... 2

Figure 1.2 Oil sands deposits in Canada (Energy Resources Conservation Board, 2009) ............ 3

Figure 1.3 Alberta oil sands locations (http://www.energy.alberta.ca/OilSands/791.asp.) ........... 4

Figure 1.4 Canada CHOPS locations and typical production history (CHOPS heavy oil 101) ..... 5

Figure 1.5 CHOPS process - wormhole placement and direction (Kelvin, 2014) ..................... 6

Figure 1.6 Schematic of typical heavy oil waterflood (NETL, 2010) ..................................... 7

Figure 1.7 Schematic of typical chemical flood (http://www.chemicalflooding.com)................ 9

Figure 1.8 Vapex process schematic (Dembicki, 2007) .......................................................... 10

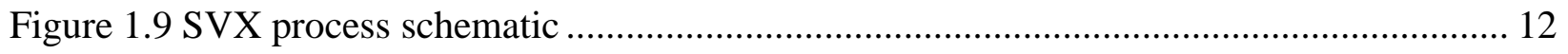

Figure 2.1 SAGD process schematic (http://jeromedowney.ca/blog/understanding-sagd) .......... 15

Figure 2.2 CSS process schematic (http://www.imperialoil.ca/en-ca/company/operations/oil-

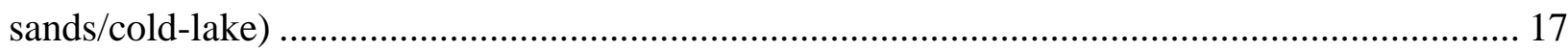

Figure 2.3 Steam flooding process schematic (http://www.steamflooding.com) ..................... 18

Figure 2.4 ISR process schematic (Harding et al., 2016) .................................................... 20

Figure 2.5 ESEIEH process schematic (Energy Solutions Overview) .................................... 22

Figure 2.6 Hydrophilic-lipophilic balance of surfactants (Image (C) MKD)............................. 23

Figure 2.7 P-x diagram comparing ideal and actual partial pressure for $\mathrm{nC}_{6}$ (Khaledi et al., 2015)

Figure 2.8 Solvent addition enhancement (Khaledi et al., 2012)......................................... 31

Figure 2.9 Physical model thermocouple grid (Jiang et al., 2012)......................................... 33

Figure 3.1 Schematic of cylindrical physical model............................................................ 45

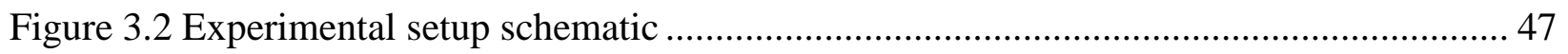

Figure 3.3 Mixing apparatus to prepare sand matrix ....................................................... 48

Figure 3.4 Schematic of experimental apparatus to measure permeability ................................ 50

Figure 3.5 Oil viscosity versus temperature ................................................................ 53

Figure 3.6 Oil density at elevated pressures and temperatures ........................................... 54

Figure 3.7 Production pressure standard deviation chart ................................................... 56

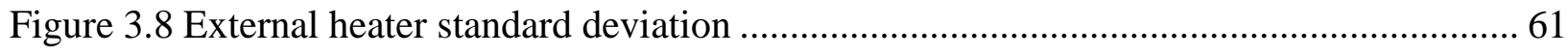

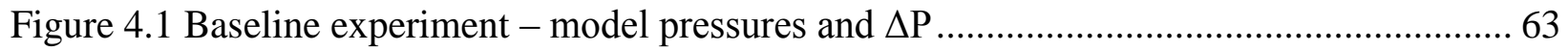


Figure 4.2 Baseline experiment cumulative and \%OOIP recovery ....................................... 64

Figure 4.3 Cumulative injected and produced water - Baseline experiment ........................... 65

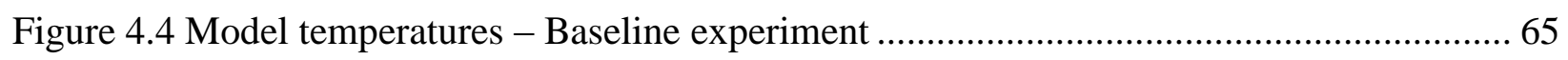

Figure 4.5 Oil recovery comparison of steam only with steam plus $\mathrm{CO}_{2}$ experiments .............. 66

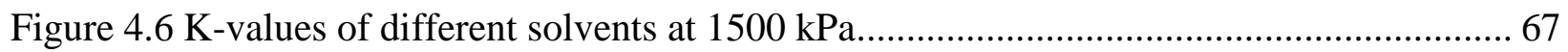

Figure 4.7 Produced oil comparison of steam only with steam plus $\mathrm{CO}_{2}$ experiments .............. 69

Figure $4.8 \%$ OOIP recovery versus $\mathrm{CO}_{2}$ concentration ................................................... 70

Figure 4.9 Differential pressure comparison of steam only with steam plus $\mathrm{CO}_{2}$ experiments ... 70

Figure 4.10 Steam plus $5.0 \mathrm{wt} \% \mathrm{CO}_{2}$ - injected and produced water ................................... 71

Figure 4.11 Steam plus $7.5 \mathrm{wt} \% \mathrm{CO}_{2}$ - injected and produced water ................................... 71

Figure 4.12 Steam plus $10.0 \mathrm{wt} \% \mathrm{CO}_{2}$ - injected and produced water .................................... 72

Figure 4.13 Steam plus $5.0 \mathrm{wt} \% \mathrm{CO}_{2}$ - injected and produced gas ...................................... 73

Figure 4.14 Steam plus $7.5 \mathrm{wt} \% \mathrm{CO}_{2}$ - injected and produced gas ....................................... 73

Figure 4.15 Steam plus $10.0 \mathrm{wt} \% \mathrm{CO}_{2}$ - injected and produced gas ...................................... 74

Figure 4.16 Oil recovery comparison of steam only with steam plus $\mathrm{C}_{3} \mathrm{H}_{8}$ experiments........... 75

Figure 4.17 Produced oil comparison of steam only with steam plus $\mathrm{C}_{3} \mathrm{H}_{8}$ experiments .......... 76

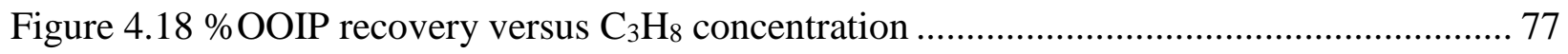

Figure $4.19 \Delta \mathrm{P}$ comparison of steam only with steam plus $\mathrm{C}_{3} \mathrm{H}_{8}$ experiments ...................... 78

Figure 4.20 Steam plus $5.0 \mathrm{wt} \% \mathrm{C}_{3} \mathrm{H}_{8}$ - injected and produced water................................. 78

Figure 4.21 Steam plus $7.5 \mathrm{wt} \% \mathrm{C}_{3} \mathrm{H}_{8}$ - injected and produced water................................... 79

Figure 4.22 Steam plus $10.0 \mathrm{wt} \% \mathrm{C}_{3} \mathrm{H}_{8}$ - injected and produced water................................. 79

Figure 4.23 Steam plus $5.0 \mathrm{wt} \% \mathrm{C}_{3} \mathrm{H}_{8}$ - injected and produced gas ..................................... 80

Figure 4.24 Steam plus $7.5 \mathrm{wt} \% \mathrm{C}_{3} \mathrm{H}_{8}$ - injected and produced gas ..................................... 81

Figure 4.25 Steam plus $10.0 \mathrm{wt} \% \mathrm{C}_{3} \mathrm{H}_{8}$ - injected and produced gas ................................... 81

Figure 4.26 Oil recovery comparison of steam only with steam plus $\mathrm{N}_{2}$ experiments............... 82

Figure 4.27 Produced oil comparison of steam only with steam plus $\mathrm{N}_{2}$ experiments .............. 83

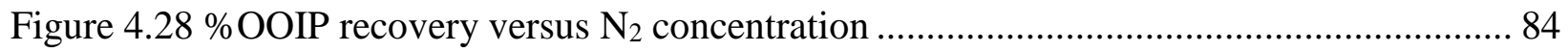

Figure $4.29 \Delta \mathrm{P}$ comparison of steam only with steam plus $\mathrm{N}_{2}$ experiments ........................... 84

Figure 4.30 Steam plus $5.0 \mathrm{wt} \% \mathrm{~N}_{2}$ - injected and produced water...................................... 85

Figure 4.31 Steam plus $7.5 \mathrm{wt} \% \mathrm{~N}_{2}$ - injected and produced water...................................... 86

Figure 4.32 Steam plus $10.0 \mathrm{wt} \% \mathrm{~N}_{2}$ - injected and produced water................................... 86 
Figure 4.33 Steam plus 5.0 wt $\% \mathrm{~N}_{2}$ - injected and produced gas ....................................... 87

Figure 4.34 Steam plus 7.5 wt $\% \mathrm{~N}_{2}$ - injected and produced gas ......................................... 87

Figure 4.35 Steam plus $10.0 \mathrm{wt} \% \mathrm{~N}_{2}$ - injected and produced gas ....................................... 88

Figure 4.36 Oil recovery comparison of steam only with steam plus $\mathrm{CH}_{4}$ experiments ............ 89

Figure 4.37 Produced oil comparison of steam only with steam plus $\mathrm{CH}_{4}$ experiments ............. 90

Figure $4.38 \%$ OOIP recovery versus $\mathrm{CH}_{4}$ concentration................................................... 90

Figure $4.39 \Delta \mathrm{P}$ comparison of steam only with steam plus $\mathrm{CH}_{4}$ experiments ......................... 91

Figure 4.40 Steam plus $5.0 \mathrm{wt} \% \mathrm{CH}_{4}$ - injected and produced water .................................. 91

Figure 4.41 Steam plus $7.5 \mathrm{wt} \% \mathrm{CH}_{4}$ - injected and produced water ................................... 92

Figure 4.42 Steam plus $10.0 \mathrm{wt} \% \mathrm{CH}_{4}$ - injected and produced water .................................. 92

Figure 4.43 Steam plus $5.0 \mathrm{wt} \% \mathrm{CH}_{4}$ - injected and produced gas....................................... 93

Figure 4.44 Steam plus 7.5 wt $\% \mathrm{CH}_{4}$ - injected and produced gas....................................... 94

Figure 4.45 Steam plus $10.0 \mathrm{wt} \% \mathrm{CH}_{4}$ - injected and produced gas...................................... 94

Figure $4.465 .0 \mathrm{wt} \%$ solvent injection - Solvent effect on \%OOIP recovery ........................... 95

Figure $4.477 .5 \mathrm{wt} \%$ solvent injection - solvent effect on \%OOIP recovery ........................... 96

Figure $4.4810 .0 \mathrm{wt} \%$ solvent injection - solvent effect on \%OOIP recovery ......................... 97

Figure 4.49 Solvent saturation properties (http://webbook.nist.gov/chemistry/fluid/) .............. 98

Figure 4.50 Solvent mol\% in injected stream versus oil recovery \%OOIP............................ 99

Figure $4.515 .0 \mathrm{wt} \%$ solvent injection - solvent effect on \%retention .................................. 100

Figure $4.527 .5 \mathrm{wt} \%$ solvent injection - solvent effect on \%retention ................................. 101

Figure $4.5310 .0 \mathrm{wt} \%$ solvent injection - solvent effect on \%retention recovery .................... 101

Figure 5.1 History-matched dead oil density comparison at atmospheric pressure and different

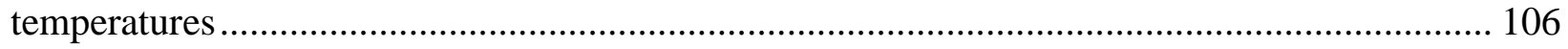

Figure 5.2 History-matched dead oil density comparison at $21^{\circ} \mathrm{C}$ and different pressures....... 107

Figure 5.3 History-matched dead oil density comparison at $35^{\circ} \mathrm{C}$ and different pressures....... 107

Figure 5.4 History-matched dead oil viscosity comparison at atmospheric pressure and different

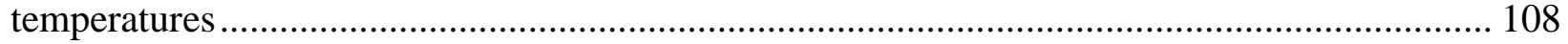

Figure 5.5 History-matched dead oil viscosity comparison at $21^{\circ} \mathrm{C}$ and different pressures ..... 109

Figure 5.6 History-matched dead oil viscosity comparison at $35^{\circ} \mathrm{C}$ and different pressures ..... 109

Figure 5.7 Some data points for $\mathrm{CO}_{2}$ and $\mathrm{N}_{2}$ solubility in bitumen, Mehrotra and Svrcek (1982) 
Figure 5.8 Gridblock distribution and porosity - Model side view 119

Figure 5.9 Gridblock distribution - Model 3D view ....................................................... 120

Figure 6.1 History-matching example ….................................................................. 125

Figure 6.2 Steam plus $5.0 \mathrm{wt} \% \mathrm{CO}_{2}$ - Oil production history-match................................... 125

Figure 6.3 Steam plus $7.5 \mathrm{wt} \% \mathrm{CO}_{2}$ - Oil production history-match.................................... 126

Figure 6.4 Steam plus $10.0 \mathrm{wt} \% \mathrm{CO}_{2}$ - Oil production history-match.................................. 127

Figure 6.5 Steam plus $5.0 \mathrm{wt} \% \mathrm{CO}_{2}$ - Gas production history-match ................................... 127

Figure 6.6 Steam plus $7.5 \mathrm{wt} \% \mathrm{CO}_{2}-$ Gas production history-match.................................... 128

Figure 6.7 Steam plus $10.0 \mathrm{wt} \% \mathrm{CO}_{2}-$ Gas production history-match................................. 129

Figure 6.8 Steam plus $5.0 \mathrm{wt} \% \mathrm{CO}_{2}-$ Water production history-match ................................ 129

Figure 6.9 Steam plus $7.5 \mathrm{wt} \% \mathrm{CO}_{2}-$ Water production history-match ............................... 130

Figure 6.10 Steam plus $10.0 \mathrm{wt} \% \mathrm{CO}_{2}-$ Water production history-match ............................ 130

Figure 6.11 Steam plus $\mathrm{CO}_{2}$ - Oil-water relative permeability curves ................................ 131

Figure 6.12 Steam plus $\mathrm{CO}_{2}$ - Oil-gas relative permeability curves .................................... 132

Figure 6.13 Steam plus 5.0 wt $\% \mathrm{~N}_{2}$ - Oil production history-match ................................... 133

Figure 6.14 Steam plus 7.5 wt $\% \mathrm{~N}_{2}$ - Oil production history-match ................................... 134

Figure 6.15 Steam plus $10.0 \mathrm{wt} \% \mathrm{~N}_{2}$ - Oil production history-match ................................. 134

Figure 6.16 Steam plus $5.0 \mathrm{wt} \% \mathrm{~N}_{2}$ - Gas production history-match .................................. 135

Figure 6.17 Steam plus $7.5 \mathrm{wt} \% \mathrm{~N}_{2}$ - Gas production history-match .................................. 136

Figure 6.18 Steam plus $10.0 \mathrm{wt} \% \mathrm{~N}_{2}$ - Gas production history-match ................................ 136

Figure 6.19 Steam plus $5.0 \mathrm{wt} \% \mathrm{~N}_{2}$ - Water production history-match................................ 137

Figure 6.20 Steam plus $7.5 \mathrm{wt} \% \mathrm{~N}_{2}$ - Water production history-match................................ 138

Figure 6.21 Steam plus $10.0 \mathrm{wt} \% \mathrm{~N}_{2}$ - Water production history-match.............................. 138

Figure 6.22 Steam plus $\mathrm{N}_{2}$ - Oil-water relative permeability curves.................................... 139

Figure 6.23 Steam plus $\mathrm{N}_{2}$ - Oil-gas relative permeability curves ...................................... 139

Figure 6.24 Steam plus $5.0 \mathrm{wt} \% \mathrm{CH}_{4}$ - Oil production history-match................................... 140

Figure 6.25 Steam plus $7.5 \mathrm{wt} \% \mathrm{CH}_{4}$ - Oil production history-match.................................. 141

Figure 6.26 Steam plus $10.0 \mathrm{wt} \% \mathrm{CH}_{4}$ - Oil production history-match................................ 142

Figure 6.27 Steam plus $5.0 \mathrm{wt} \% \mathrm{CH}_{4}-$ Gas production history-match ................................ 142

Figure 6.28 Steam plus $7.5 \mathrm{wt} \% \mathrm{CH}_{4}-$ Gas production history-match ................................ 143

Figure 6.29 Steam plus $10.0 \mathrm{wt} \% \mathrm{CH}_{4}$ - Gas production history-match .............................. 143 
Figure 6.30 Steam plus $5.0 \mathrm{wt} \% \mathrm{CH}_{4}-$ Water production history-match .............................. 144

Figure 6.31 Steam plus $7.5 \mathrm{wt} \% \mathrm{CH}_{4}-$ Water production history-match ............................. 145

Figure 6.32 Steam plus $10.0 \mathrm{wt} \% \mathrm{CH}_{4}-$ Water production history-match ............................ 145

Figure 6.33 Steam plus $\mathrm{CH}_{4}$ - Oil-water relative permeability curves ................................ 146

Figure 6.34 Steam plus $\mathrm{CH}_{4}$ - Oil-gas relative permeability curves ................................... 146 


\section{NOMENCLATURE}

A Cross sectional area, $\mathrm{m}^{2}$

$A d_{i} \quad$ Adsorbed concentration, $\mathrm{kg} / \mathrm{m}^{3}$

$D_{i j} \quad$ Molecular diffusion, $\mathrm{m}^{2} / \mathrm{s}$

$D_{i j}^{*} \quad$ Molecular diffusion of component $i$ in phase $j, \mathrm{~m}^{2} / \mathrm{s}$

$D_{j i} \quad$ Component dispersibilities, $\mathrm{m}^{2} / \mathrm{s}, j=\mathrm{o}, \mathrm{w}, \mathrm{g}, \mathrm{o}=\mathrm{oil}$, w=water, $\mathrm{g}=\mathrm{gas}$

$F_{j k} \quad$ Tortuosity experienced by phase $j$ in $k$ direction

$H A_{C D}$ Rate of heat transferred by conduction to/from adjacent aquifer

$H A_{C V}$ Rate of heat transferred by convection to/from adjacent aquifer

$H_{j} \quad$ Enthalpy, joules/kg, $j=\mathrm{o}, \mathrm{w}, \mathrm{g}, \mathrm{o}=\mathrm{oil}, \mathrm{w}=$ water, $\mathrm{g}=$ gas

$H_{r k} \quad$ Enthalpy of reaction $k$

$J_{i j k} \quad$ Flux of component $i$ in phase $j$ in $k$ direction, moles $/ \mathrm{m}^{2} / \mathrm{sec}$

k Absolute permeability, Darcy

$k_{\mathrm{rj}} \quad$ Relative permeability of fluid $j$

$K_{\mathrm{T}} \quad$ Thermal transmissibility at the interface between two regions

$L \quad$ Length, $\mathrm{m}$

$n_{f} \quad$ Number of neighbouring regions or gridblock faces

$P \quad$ Pressure, $\mathrm{kPa}$

$\mathrm{P}_{\mathrm{c}} \quad$ Critical pressure, $\mathrm{kPa}$

$q \quad$ Volumetric flow rate, $\mathrm{m}^{3} / \mathrm{s}$

$q a q_{\mathrm{w} k}$ Volumetric water flow rate through a block face $k$ to/from the adjacent aquifer

R Gas constant, $\mathrm{j} /(\mathrm{mol}$ Kelvin $)$

$r_{k} \quad$ Volumetric rate of reaction $k$

$S_{\mathrm{g}} \quad$ Gas saturation

$S_{\mathrm{o}} \quad$ Oil saturation

$S_{\mathrm{w}} \quad$ Water saturation

$S_{j} \quad$ Saturation of phase $j$

$S_{k i} \quad$ Reactant stoichiometry coefficient of component $I$ in reaction $k$

$S_{k i}^{\prime} \quad$ Product stoichiometry coefficient of component $I$ in reaction $k$ 


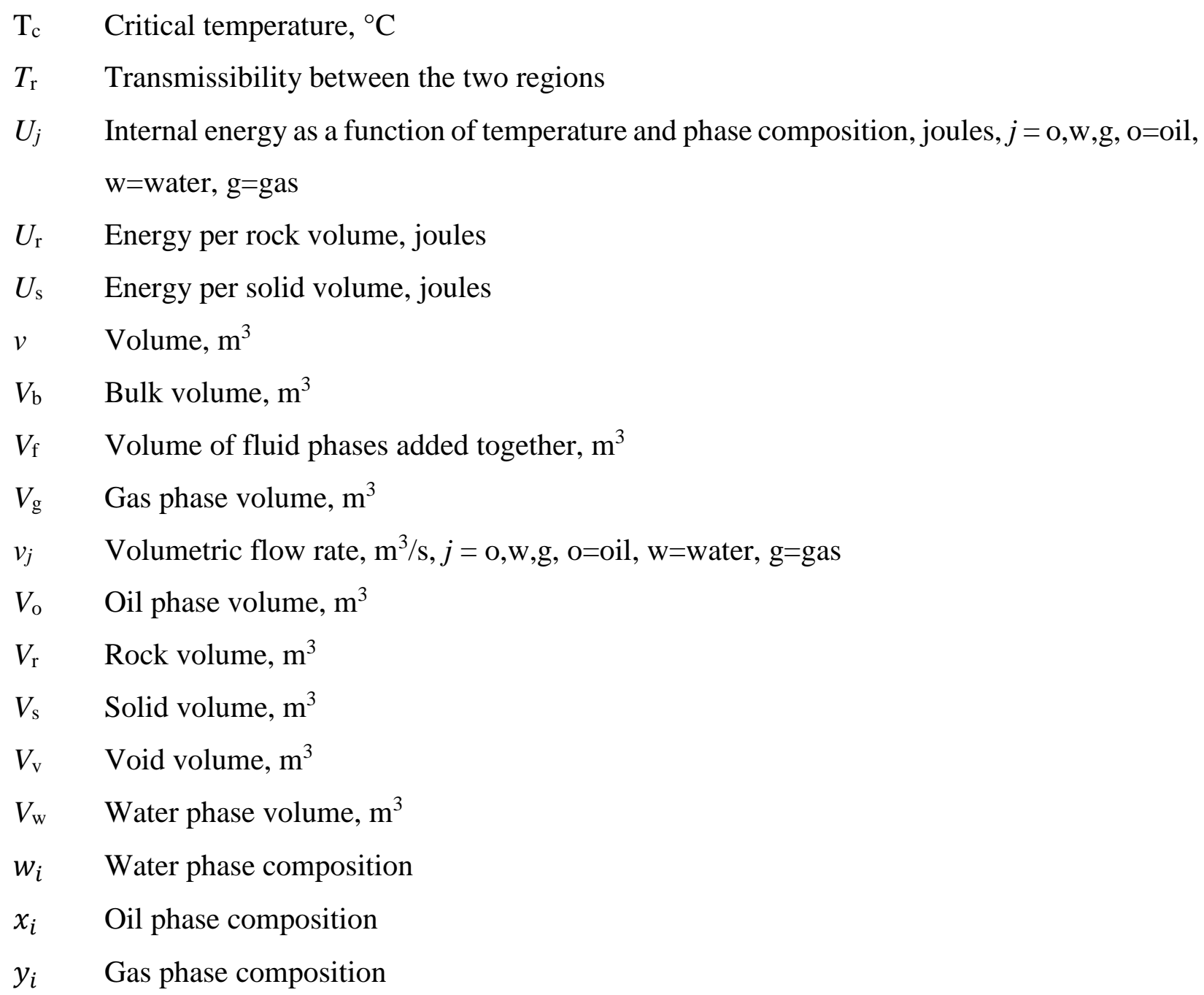

\section{Greek Symbols}

$\Omega_{a} \quad$ Interaction coefficient

$\Omega_{b} \quad$ Interaction coefficient

$v_{j k} \quad$ Absolute value of interstitial velocity of phase $j, \mathrm{~m} / \mathrm{s}$

$\alpha_{j k} \quad$ Mechanical dispersivity for phase $j$ in direction $k, \mathrm{~m}^{2} / \mathrm{s}$

$\mu_{j} \quad$ Viscosity of fluid $j, \mathrm{cP}$

$\Delta \Phi_{j} \quad$ The value at the node of the adjacent region minus the value at the node of the current region of interest. A positive value for $\Delta \Phi_{j}$ represents inflow; a negative value gives outflow.

$\mu \quad$ Dynamic viscosity, $\mathrm{cP}$ 
$\phi \quad$ Porosity

$\omega \quad$ Acentric factor

$\rho_{j} \quad$ Fluid density, $\mathrm{kg} / \mathrm{m}^{3}, j=\mathrm{o}, \mathrm{w}, \mathrm{g}, \mathrm{o}=\mathrm{oil}, \mathrm{w}=$ water, $\mathrm{g}=\mathrm{gas}$

\section{Acronyms}

API - American petroleum institute

ASP - Alkali surfactant polymer

CHOPS - Cold heavy oil production with sand

CSS - Cyclic steam stimulation

DME - Dimethyl ether

ES-SAGD - Expanded solvent steam assisted gravity drainage

HLB - Hydrophilic-lipophilic balance

ISR - In-situ reflux

MBE - Matrix bypass event

PV - Pore volume

$\mathrm{RF}$ - Radio frequency

SAGD - Steam assisted gravity drainage

SAP - Solvent assisted process

SA-SAGD - Solvent assisted steam assisted gravity drainage

SVX - Solvent vapour extraction

Vapex - Vapour extraction of heavy oil 


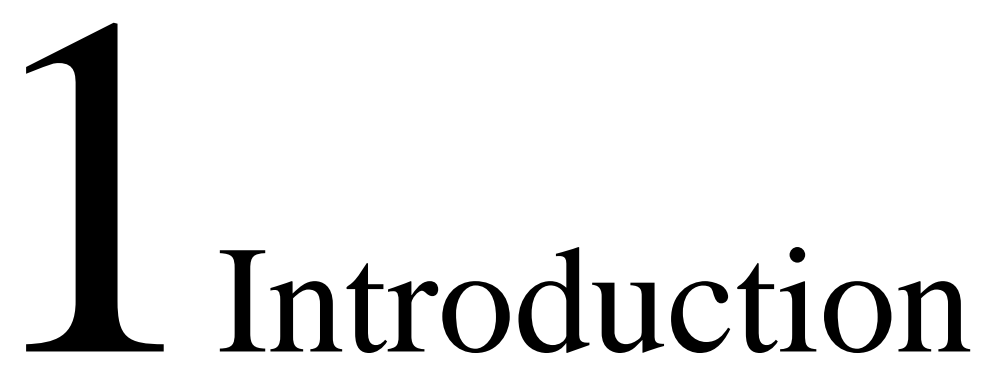

This chapter provides a basic definition of crude oil, and a brief discussion about heavy oil and bitumen resources in Canada and around the world. Also included is a brief introduction to state of the art cold heavy oil recovery technologies.

\subsection{What is Crude Oil?}

Crude oil is a naturally occurring fluid, composed of hydrocarbon deposits and other organic materials. Crude oil can be refined to produce usable products such as gasoline, diesel and various forms of petrochemicals. Heavy oil is a non-renewable energy resource and is usually found alongside other resources, such as natural gas and saline water. The viscosity and relative weight of crude oil varies and define its classification. The properties of the crude oil vary in terms of proportion of its hydrocarbon elements - saturate, aromatic, resin, and asphaltene (SARA) fractions, acid number and sulfur contents, etc.

Generally, crude oil is classified into light, medium, heavy and extra heavy or bitumen. A light crude oil is defined as having an API (American Petroleum Institute) gravity value higher than $31.1^{\circ} \mathrm{API}$. Medium oil is defined as having API gravity between $22.3^{\circ} \mathrm{API}$ and $31.1^{\circ} \mathrm{API}$. Heavy oil is defined as having API gravity below $22.3^{\circ} \mathrm{API}$. Extra heavy oil or bitumen is defined having API gravity below $10^{\circ} \mathrm{API}$.

Light, sweet crude is more expensive than heavier oil, because it requires less processing and creates a slate of goods with a greater percentage of value-added products, such as gasoline, diesel, 
and aviation fuel. Heavier crude typically sells at a discount to lighter, sweeter grades because it yields a greater percentage of lower-value-added products with simple distillation and requires additional processing to produce lighter products.

Typically, light and sweet crude oils are classified as conventional oil reserves, and heavy oil and bitumen are classified as unconventional oil reserves. The world's unconventional oil reserves exceed their conventional counterparts, and are much more difficult and expensive to process. Figure 1.1 shows a chart with percentage of world oil reserves by region (US Energy Information Administration, 2007).

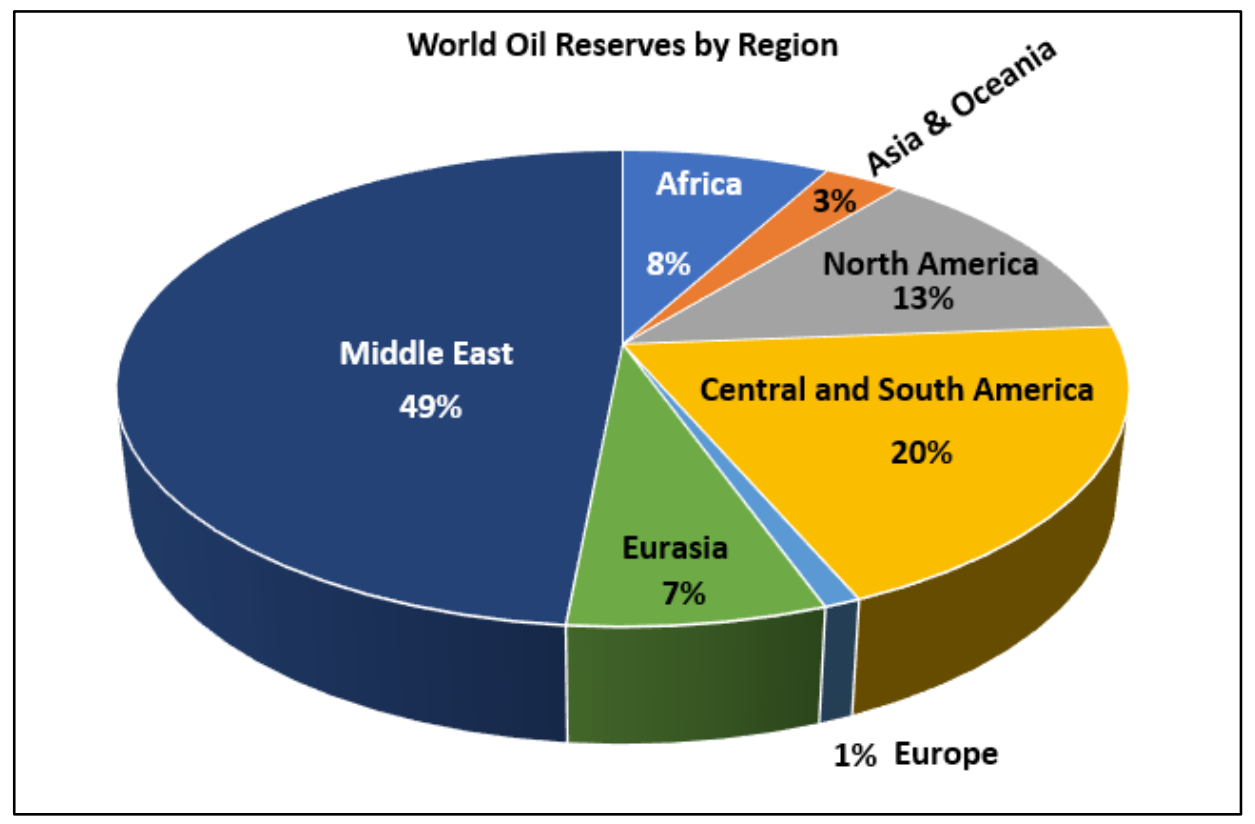

Figure 1.1 World oil reserves by region (from US Energy Information Administration, 2007)

\subsection{Natural Bitumen and Extra Heavy Oil}

Natural bitumen and extra heavy oil are characterized by high viscosity, high density (low API gravity), and high concentrations of nitrogen, oxygen, sulphur, and heavy metals. These characteristics result in higher costs for extraction, transportation, and refining than are incurred with conventional oil. Despite these costs and technical challenges, major international oil 
companies have found it desirable to acquire, develop, and produce these resources in increasing volumes. Large in-place resource volumes provide a reliable, long-term flow of liquid hydrocarbons and provide substantial payoff for any incremental improvements in recovery.

Offshore natural bitumen deposits have not been reported in the literature. Bitumen is present both in clastic and carbonate reservoir rocks and commonly in small deposits at, or near, the earth's surface. Natural bitumen deposits have been mined since antiquity for use as sealants and paving materials. In a few places, such deposits are extremely large, both in areal extent and in the resources they contain, most notably those in the northern Alberta portion of the Western Canadian Sedimentary Basin. The three Alberta oil sand areas (Figure 1.2)—Athabasca, Peace River, and Cold Lake-together contain 1.73 trillion barrels of discovered bitumen in place (Energy Resources Conservation Board, 2009), representing two-thirds of the world's total. More than $40 \%$ of the crude oil and bitumen produced in Canada in 2008 came from the Alberta natural bitumen deposits.

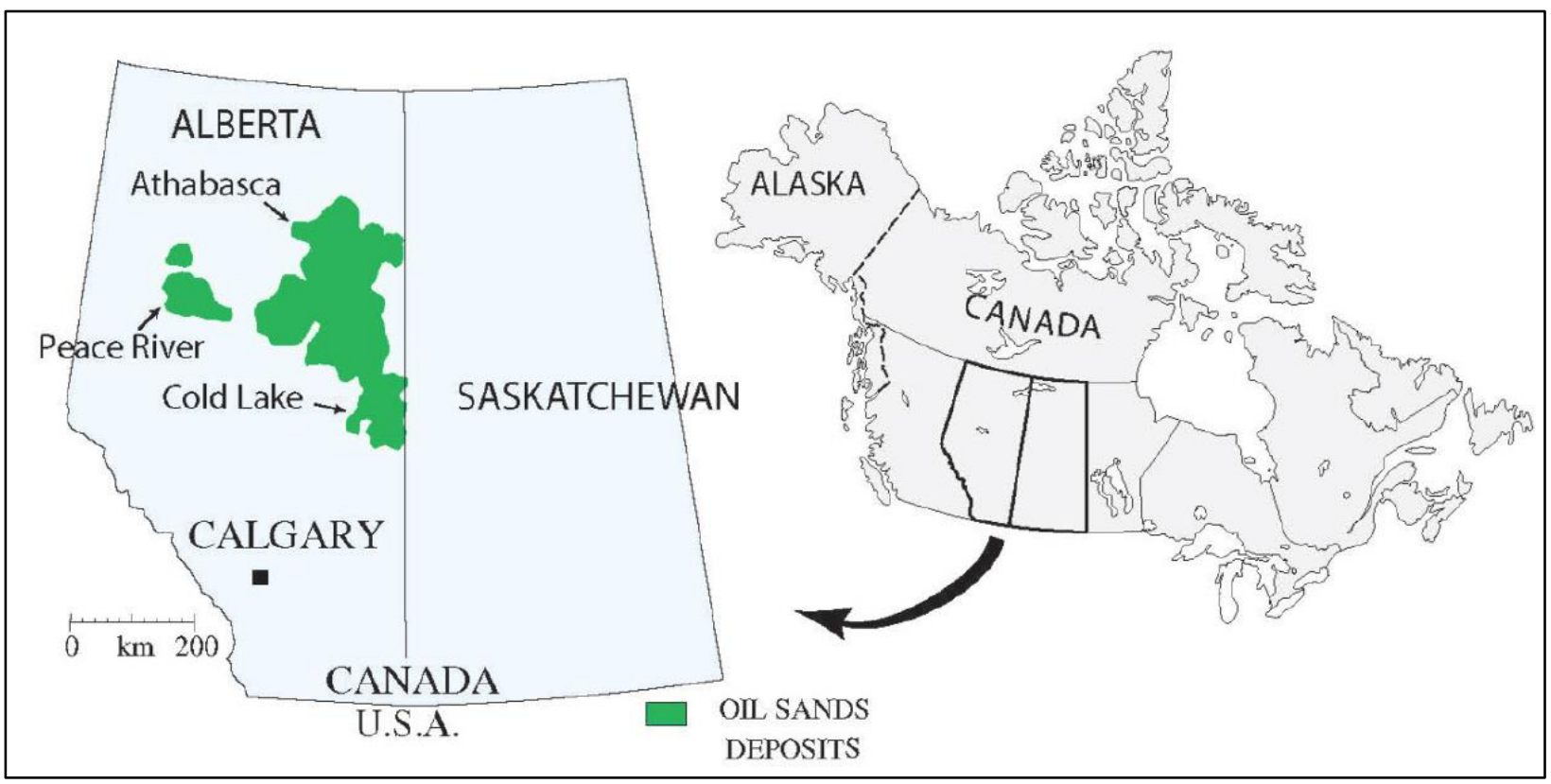

Figure 1.2 Oil sands deposits in Canada (Energy Resources Conservation Board, 2009)

Canadian energy production has almost doubled since 1980. Out of the total reserves, about 165 billion barrels can be recovered from the oil sands using today's technology. As of December 2016, 
Canada is third after Venezuela and Saudi Arabia in terms of global oil reserves. Over the last 35 years, Canadian crude oil production has increased by more than 2.4 million barrels/day due to the growth in supply from the oil sands (https://www.capp.ca/canadian-oil-and-natural-gas/oil-sands).

Alberta's oil sands underlie 142,200 square kilometres $\left(\mathrm{km}^{2}\right)$ of land in the Athabasca, Cold Lake and Peace River areas of northern Alberta (Figure 1.3). Reserves shallow enough to mine (up to 75 meters) are found only within the Athabasca oil sands area. Surface mineable area (SMA) equals about $4,800 \mathrm{~km}^{2}$ and accounts for about 3.4 per cent of total oil sands area (http://www.energy.alberta.ca/OilSands/791.asp.).

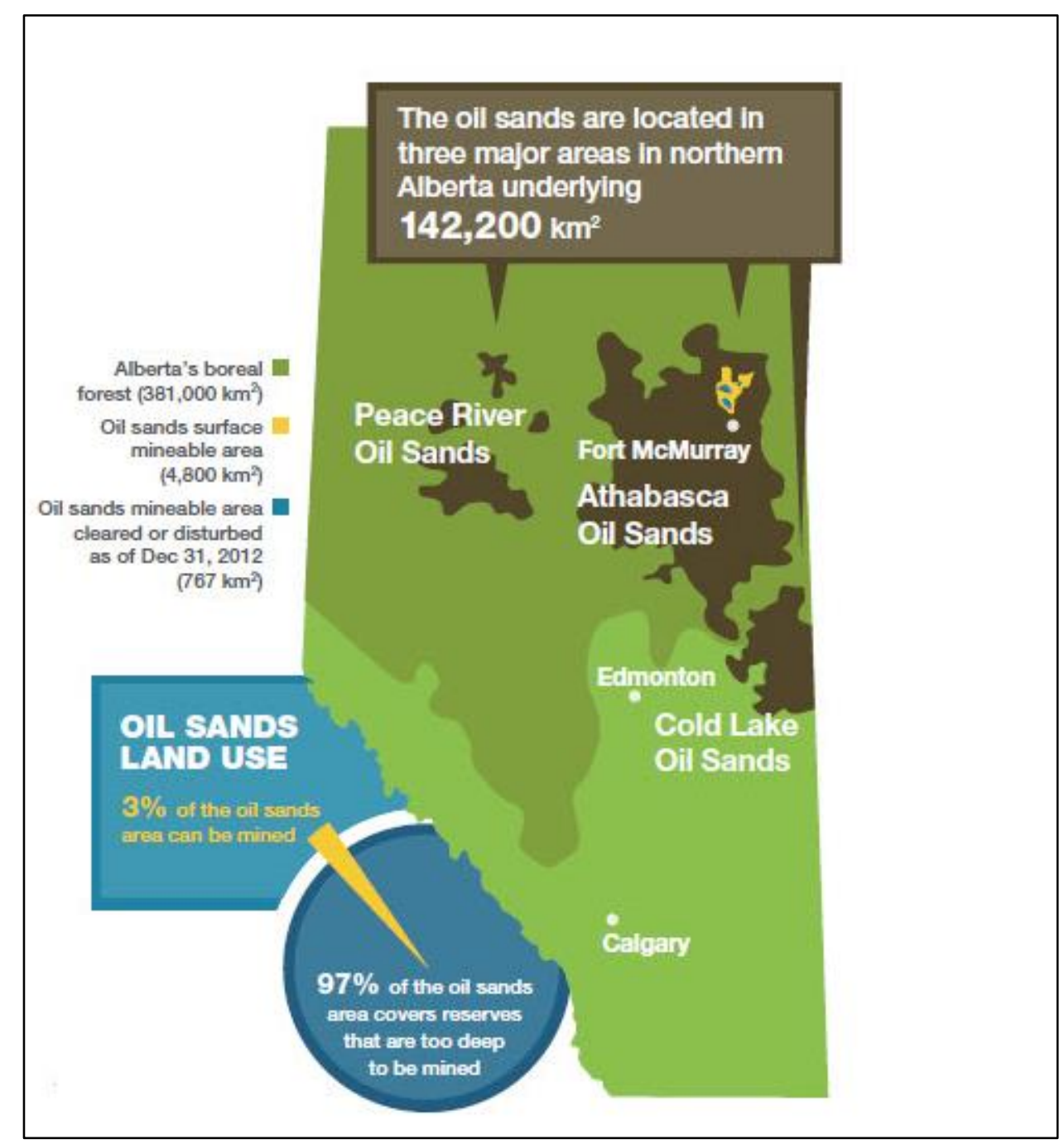

Figure 1.3 Alberta oil sands locations (http://www.energy.alberta.ca/OilSands/791.asp.) 


\subsection{Cold Heavy Oil and Bitumen Recovery Technologies}

There is a long history of recovery technologies developed and implemented to recover the heavy oil and bitumen present in Canadian reservoirs. These technologies can be divided into two main categories of (a) thermal and (b) non-thermal or cold. The non-thermal or cold technologies refer to those that do not use heat to produce oil. The most promising non-thermal enhanced oil recovery processes are briefly described in the subsections below.

\subsubsection{Cold Heavy Oil Production with Sands (CHOPS)}

The Lloydminster area straddling the Alberta-Saskatchewan border contains vast amounts of heavy oil deposits in thin unconsolidated formations (Gokhan et al., 2013). Cold heavy oil production with sand (CHOPS) is a popular and commonly used process in Canadian heavy oil reservoirs. The ultimate recovery from CHOPS ranges from 5 to 15 per cent of the original oil in place (\% OOIP). Figure 1.4 shows the map of the area where CHOPS is being implemented in Canada, along with a typical CHOPS production history (CHOPS heavy oil 101).

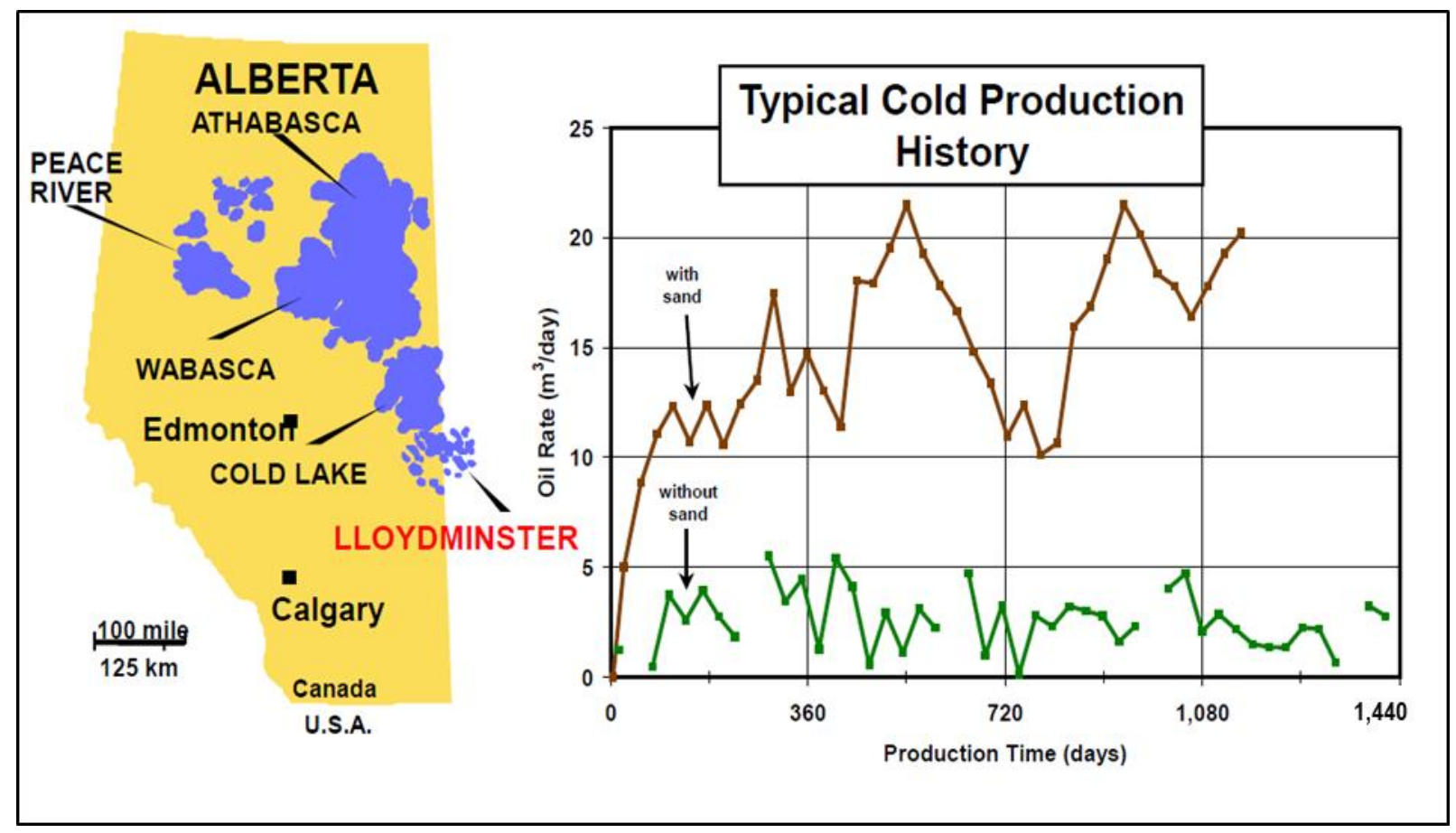

Figure 1.4 Canada CHOPS locations and typical production history (CHOPS heavy oil 101) 
CHOPS is defined as primary heavy oil production that involves the deliberate initiation of sand influx into a perforated oil well, and the continued production of substantial quantities of sand along with the oil. CHOPS requires management of large quantities of sand in all phases of production. During CHOPS, different physical processes occur in the reservoir, for example, foamy oil behaviour, massive stress distribution, liquefaction of sand, flow of four-phase slurry and, most importantly, generation and growth of wormholes. When the wormholes first propagate into the formation, they are filled with sand. Eventually, as the wormhole get longer, more oil is drained into the wormholes and an open channel develops (Tremblay, 2005). This open channel is surrounded by a dilated zone that has permeability and porosity that are 5 to 10 times higher than the reservoir permeability and porosity. The open channel diameter can range from .05 to 0.1 meters and the dilated zone diameter can range from 0.5 to 1.0 meters (Tremblay, 2005). Figure 1.5 shows a schematic of a vertical well with wormholes propagated in the horizontal direction (Kelvin, 2014).

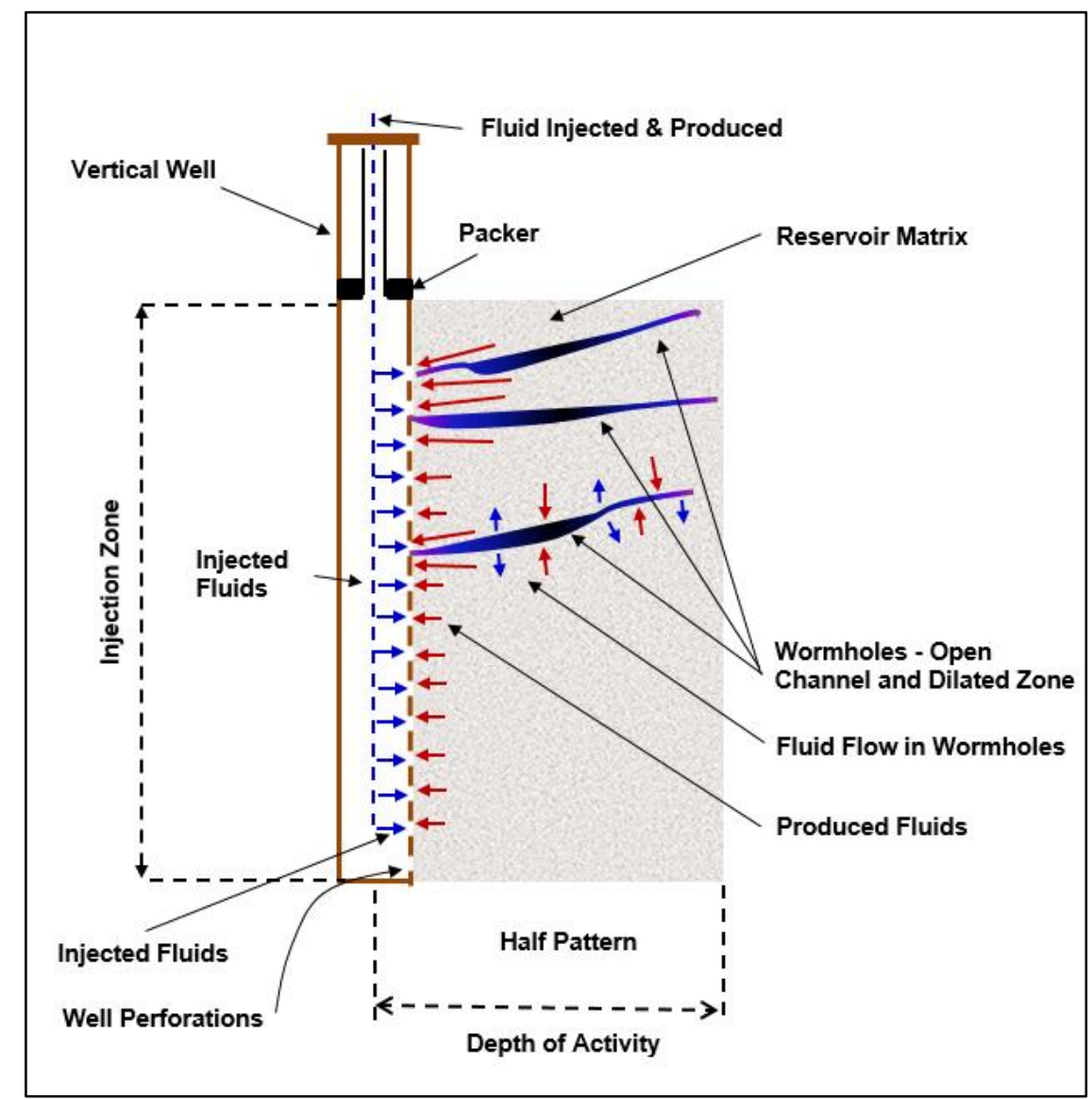

Figure 1.5 CHOPS process - wormhole placement and direction (Kelvin, 2014) 
The presence of wormholes is one of the reasons that the recovery from this process hardly exceeds $10 \%$ OOIP. These wormholes can connect to water channels and, once that happens, the oil cut (fraction of oil in the produced fluid) drops significantly and the high water cut rises steeply. On the other hand, the presence of these wormholes provides access to the reservoir, which provides an opportunity for application of post-CHOPS processes to recover the remaining still- high oil volumes (about 90\% OOIP) from CHOPS reservoirs.

\subsubsection{Heavy Oil Waterflooding}

Waterflooding is another commonly used heavy oil recovery process implemented in Canadian reservoirs. Heavy oil waterfloods have been operating in the petroleum industry for more than 50 years. In Western Canada, of the 5201 million $\mathrm{m}^{3}$ of heavy oil in place in Alberta and Saskatchewan, over $24 \%$ is recovered by more than 200 waterflood operations (Brice and Renouf, 2008). This is primarily a displacement process in which water is injected from one vertical well, displaces the oil in the reservoir, and sweeps it towards the production vertical well (Figure 1.6).

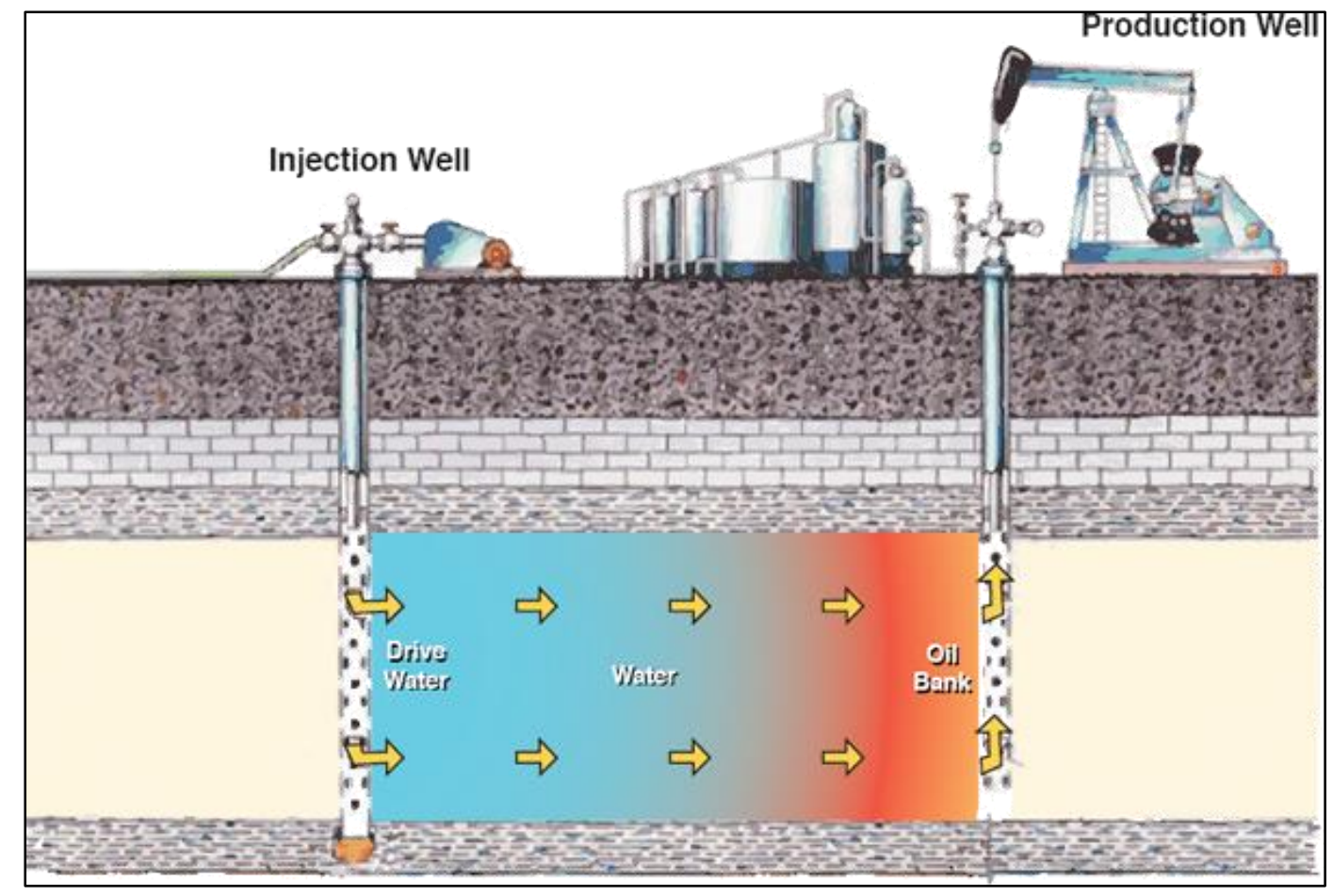

Figure 1.6 Schematic of typical heavy oil waterflood (NETL, 2010) 
Practices for waterflooding of conventional light oils were initially researched in 1940s by Buckley and Leverett (1942). However, heavy oil waterfloods perform differently from conventional waterfloods; specifically, this process in heavy oil is more challenging because of the predominance of water channeling. The high viscosity of the oil, the low unconsolidated strength of the rock, and high permeability streaks within the reservoir all contribute to the development of matrix bypass events (MBE). Once MBEs occur, the water cut tends to rise high, with an accompanying decrease in oil cut (Brice et al., 2014).

\subsubsection{Chemical Flooding Process}

A significant amount of oil remains in the reservoir at the end of the waterflooding process - often more than $50 \%$, or even more for medium and heavy oil fields. Chemical enhanced oil recovery (EOR) is one of the most widely adopted EOR processes and has been in use since the 1970s. Chemical EOR requires a minimal additional infrastructure compared to most other EOR processes (low capital cost). In chemical EOR, one or several chemicals are added to the injected fluid (water) to improve the viscosity ratio of the displacing and displaced fluids (and hence improve sweep efficiency), and reduce interfacial tension (and reduce capillary entrapment). In addition, some chemical processes also target changing the wettability of the reservoir (typically to make it more water wet) and partially solubilize the oil to facilitate its transport. Figure 1.7 (http://www.chemicalflooding.com) shows a simple schematic of the chemical ASP (alkalinesurfactant-polymer) flooding process. As shown in the figure, from two vertical wells, after a preflush to condition the reservoir, a micellar fluid is injected that releases the oil. This is followed by sequential injection of i) polymer solution for mobility control, ii) fresh water buffer to protect the injected polymer, and iii) driving fluid (water).

The three most commonly used classes of chemicals in a chemical EOR process are polymers, surfactants and alkali. Various combinations of these three types of chemicals can be used, depending on the application.

Chemical flooding can enhance the displacement efficiency of waterflooding that is usually divided into two categories: microscopic (pore level) sweep efficiency and macroscopic (areal, 
vertical + horizontal) sweep efficiency. Pore level displacement efficiency is limited by capillary forces and can be improved using surface active agents (such as surfactants). Macroscopic (areal) sweep efficiency is limited by viscous fingering and early breakthrough of the injected fluid, due to the difference in viscosity between oil and water as well as due to reservoir heterogeneity, and can be improved using polymers.

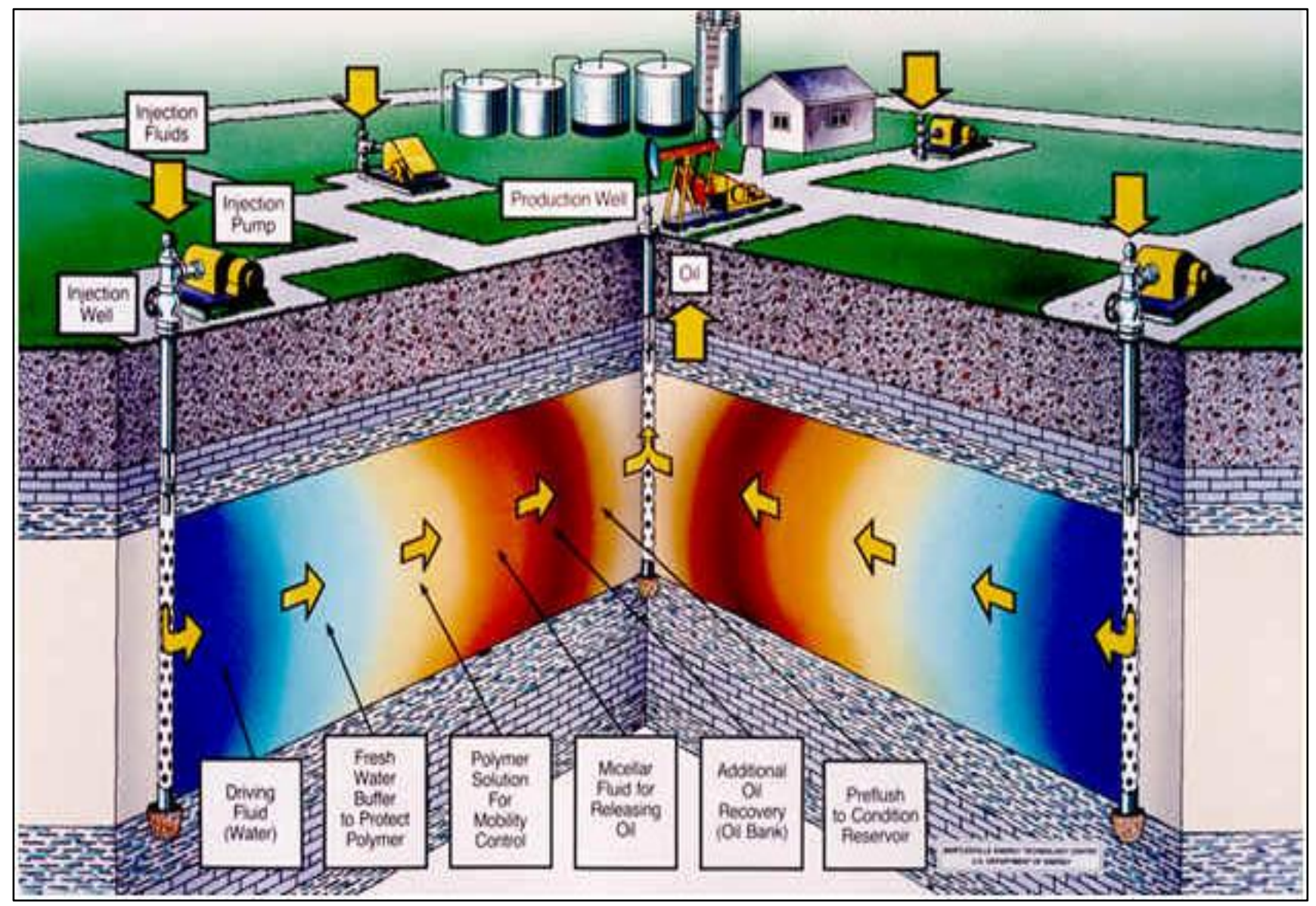

Figure 1.7 Schematic of typical chemical flood (http://www.chemicalflooding.com)

Polymers used in EOR are very large, water-soluble molecules, typically consisting of different monomers. Because of their size, polymers can significantly increase the viscosity of the water at very low concentrations (commonly used concentrations are in the range of 1,000-3,000 ppm). In addition, polymers tend to moderately reduce permeability of the formation to water, while having less effect on oil permeability. All this helps to reduce viscous fingering and improve sweep (macroscopic) displacement efficiency. 
The most commonly used polymers are based on the acrylamide monomers (polyacrylamides). Another class of polymers that are sometimes used are biopolymers, such as xanthan or guar gum. Polyacrylamides are usually cheaper to produce and are more consistent in quality, and have better resistance to bacterial degradation. Polyacrylamides are used in more than $80 \%$ of EOR applications worldwide.

\subsubsection{Vapour Extraction of Heavy Oil (Vapex)}

The first, but still raw, idea for the Vapex process is attributed to Allen (1974); it varied the cyclic steam stimulation (CSS) process by alternating steam and solvents (butane and propane). The refined Vapex concept is the injection of a gaseous blend containing two components, one as carrier gas and the other as solvent.

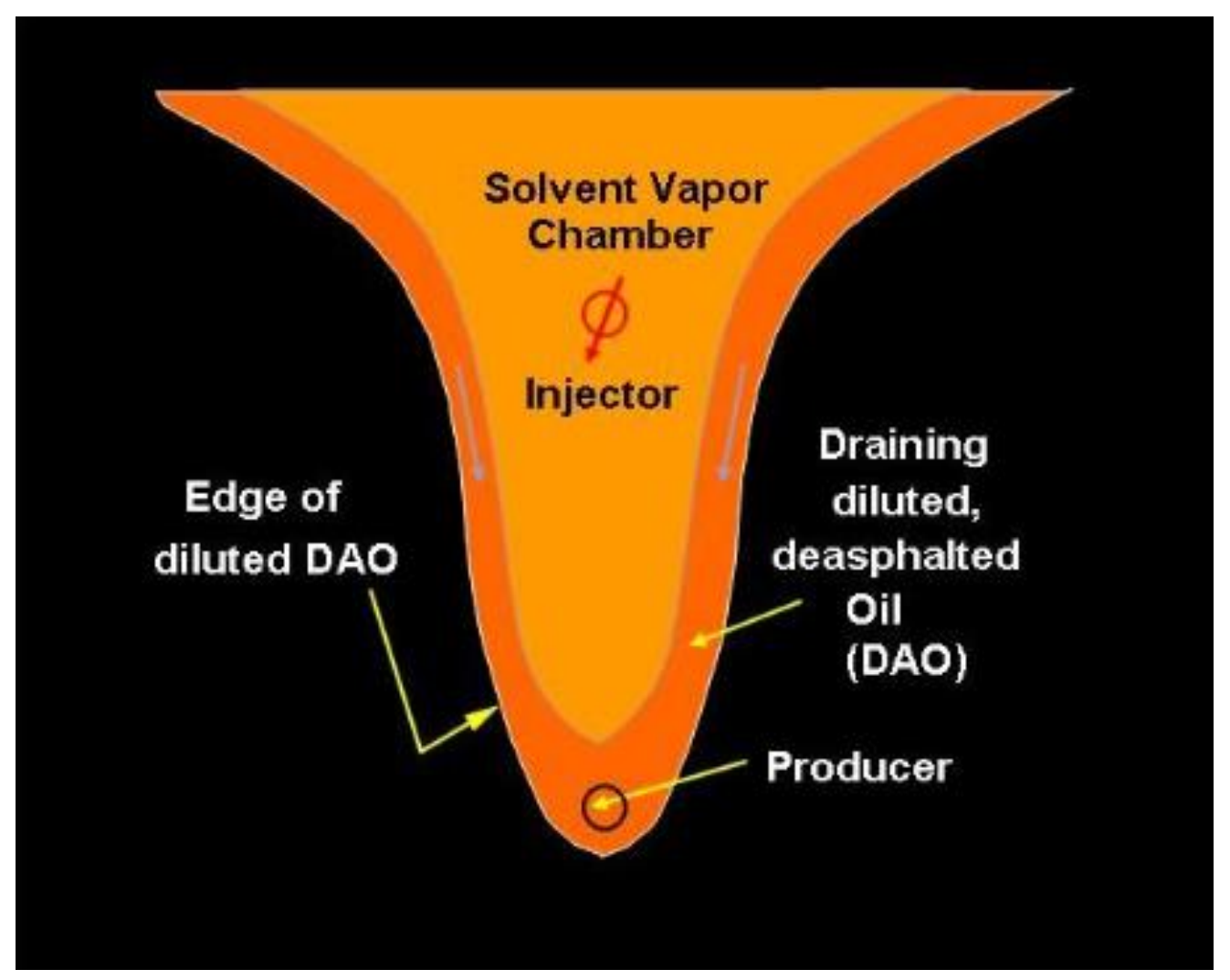

Figure 1.8 Vapex process schematic (Dembicki, 2007) 
A simple schematic of this process is shown in Figure 1.8 (Dembicki, 2007). In Vapex, a pure hydrocarbon solvent or its mixture with other solvents or non-condensable gases is injected into a horizontally placed injection well. The injection conditions are designed such that the solvent is very close to its dewpoint in the vapour state. After injection, a solvent chamber is formed above the injection well which rises and expands with time. At the edge of the chamber, solvent diffuses into the oil and reduces its viscosity, and the reduced-viscosity oil drains towards the production well that is placed parallel to the injection well. The distance between the injection and production wells needs to be about 5 meters and so Vapex is applied to thicker reservoirs with pay zone thickness of 15-plus meters.

In Vapex, three distinct stages are involved in the molecular diffusion phenomenon of an injected gas into heavy oil. First, the gas moves towards the oil-gas interface, then the gas penetrates the interface, and finally the penetrated gas diffuses in the oil body. Accurate diffusion data for solvent-heavy oil systems are necessary to determine the amount of gas required, the required time and the rate of oil production (Butler and Mokrys, 1990). The first field pilot for Vapex was initiated by Nexen Inc. in 2004 in the Winter area of Saskatchewan (Winter Pilot Expansion SPRI Final Report).

\subsubsection{Solvent Vapour Extraction (SVX)}

The idea of solvent vapour extraction was first introduced by Knorr and Imran (2011). The operational concept of SVX is similar to that of Vapex, with the major difference being the placement of horizontal injection/production wells that makes SVX suitable for even thin reservoirs featuring less than 8 meters of reservoir thickness. A typical lateral SVX process includes laterally and vertically separated horizontal injection and production wells (Figure 1.9).

Similar to Vapex, depending on reservoir petro-physical properties, a pure hydrocarbon solvent or its mixture with a non-condensable gas is injected into the reservoir. Upon solvent injection, solvent conduits between the injection and production wells start to form and, with the passage of time, these solvents conduits convert into solvent chambers (Knorr and Imran, 2012). On the edge of a solvent chamber, the solvent dissolves into the heavy oil and reduces its viscosity and, under 
the combined effects of gravity and differential pressure between the injection and production wells (shown with red and blue solid circles in Figure 1.9), this reduced-viscosity oil flows towards the production well through oil drainage conduits. During solvent injection process the solvent mass transfer into the heavy oil is controlled by a concentration-dependent solvent dispersion process (Abukhalifeh et al., 2009).

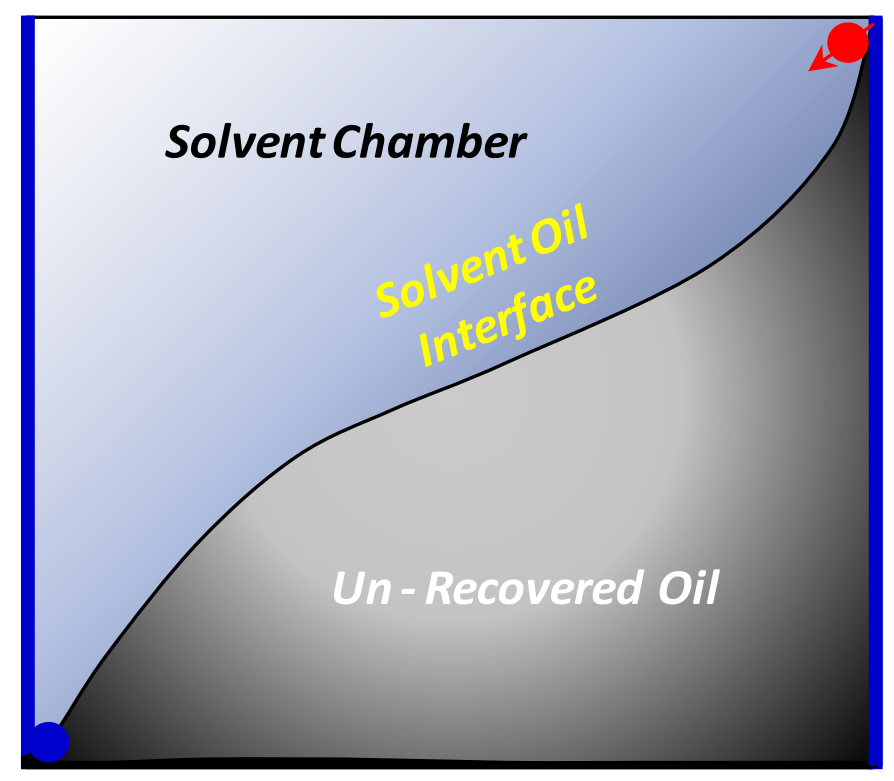

Injector

- Producer

Figure 1.9 SVX process schematic

\subsubsection{DME Injection Process}

Hydrocarbon solvents are generally used for mass transfer into heavy oil and bitumen. Liquid solvents often include paraffins such as pentane or heptane, or aromatic solvents such as toluene to avoid precipitation of asphaltenes. Liquid propane may also be used in colder reservoirs where it liquefies at low pressures. The major impediment to using hydrocarbon solvents is their cost; with higher oil prices, the cost increases accordingly for liquefied petroleum gas (LPG) products. Alternative solvents, which have similar mass transfer rates and solubility in these viscous oil systems, are of considerable benefit to these processes.

One such option is di-methyl ether (DME); this chemical is often described as a "synthetic LPG." DME can be manufactured from fossil fuels or from renewable sources such as waste, pulp/paper 
mill residues and agricultural products (Taupy, 2007). DME is an isomer of ethanol and, like ethanol, it is soluble in water. The fact that it is soluble in both water and oil makes it a unique agent. Both molecular mass and boiling point of DME are, respectively, in between those of propane and butane. According to experimental work at the University of Calgary, DME showed high mass transfer rate into Peace River and Grosmont bitumens and higher swelling of the oil phase compared to propane (Diedro et al., 2015). DME has similar vapour pressure and density to LPG products such as propane, so it can be transported and handled similarly to hydrocarbon solvents.

The above-cited enhanced oil recovery technologies are applicable to heavy oil reservoirs. For bitumen reservoirs with viscosities in the millions of centipoise and with API gravity below $10^{\circ} \mathrm{API}$, thermal processes are more suitable. The next chapter presents a brief introduction to state of the art thermal technologies, along with a comprehensive literature survey that focuses on physical and numerical modelling and field experience related to hybrid SAGD, which is the focus of the present work. 


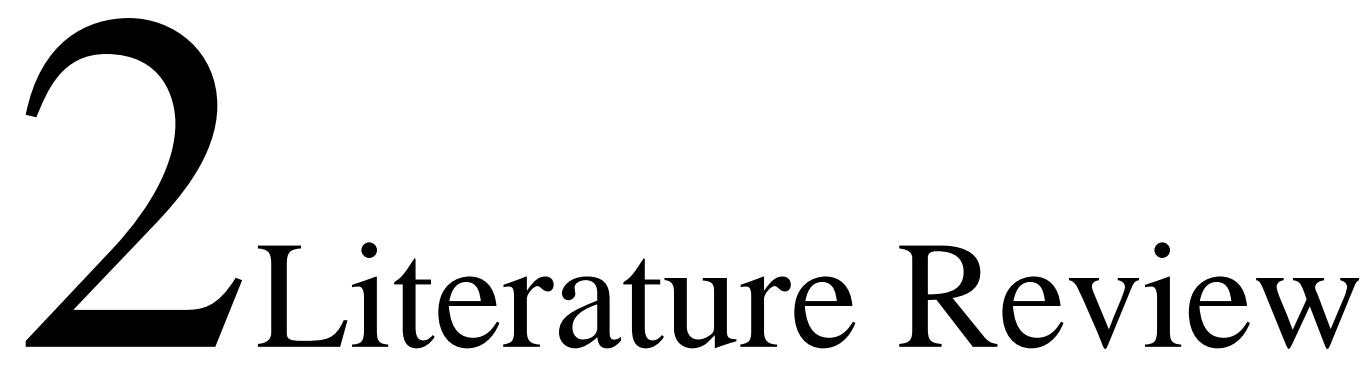

Hybrid steam-assisted gravity drainage (SAGD) is a thermal enhanced oil recovery process using a SAGD well configuration (i.e. a pair of horizontal injection and production well) in which gaseous or liquid solvents are co-injected along the steam. Depending on the type of solvent being injected and its timing of injection, researchers have proposed different names for hybrid SAGD, for example, solvent-aided process (SAP), expanded solvent ES-SAGD, and SAGD with noncondensable gases.

This chapter provides a brief description of the most promising thermal enhanced oil recovery processes. A comprehensive literature survey is also presented, which focuses on physical and numerical modelling and field experience related to hybrid SAGD — the main focus of the present work.

\subsection{Thermal Heavy Oil and Bitumen Recovery Technologies}

Thermal technologies refer to those that produce oil with the use of heat. In thermal processes, oil viscosity reduction is one of the primary recovery mechanisms. The most promising thermal enhanced oil recovery processes are briefly described below.

\subsubsection{Steam-Assisted Gravity Drainage (SAGD)}

Steam-assisted gravity drainage SAGD as shown in Figure 2.1 was developed by Butler (1985) to take advantage of gravity drainage with the recent innovation of horizontal wells. SAGD involves 
two vertically separated horizontal wells, with the injector drilled directly above the producer. Typical distance between the wells is $3-5$ metres. In this process, steam is continuously injected into the formation, where it rises, forming a steam chamber, while heated oil and steam condensate drain down by gravity along the walls of the steam chamber and into the production well. The initial start-up requires pre-heating in both wells, which is done by circulating steam down tubing and condensate up the annulus. The pre-heating is required to mobilize the oil between injection and production wells. This oil mobilization, or, in other words communication between the two wells, is essential for starting the process. After a sufficient period of injection, a subcooled region containing liquid is maintained above the producing well to prevent steam from short-circuiting between the injector and producer.

\section{SAGD TECHNOLOGY}

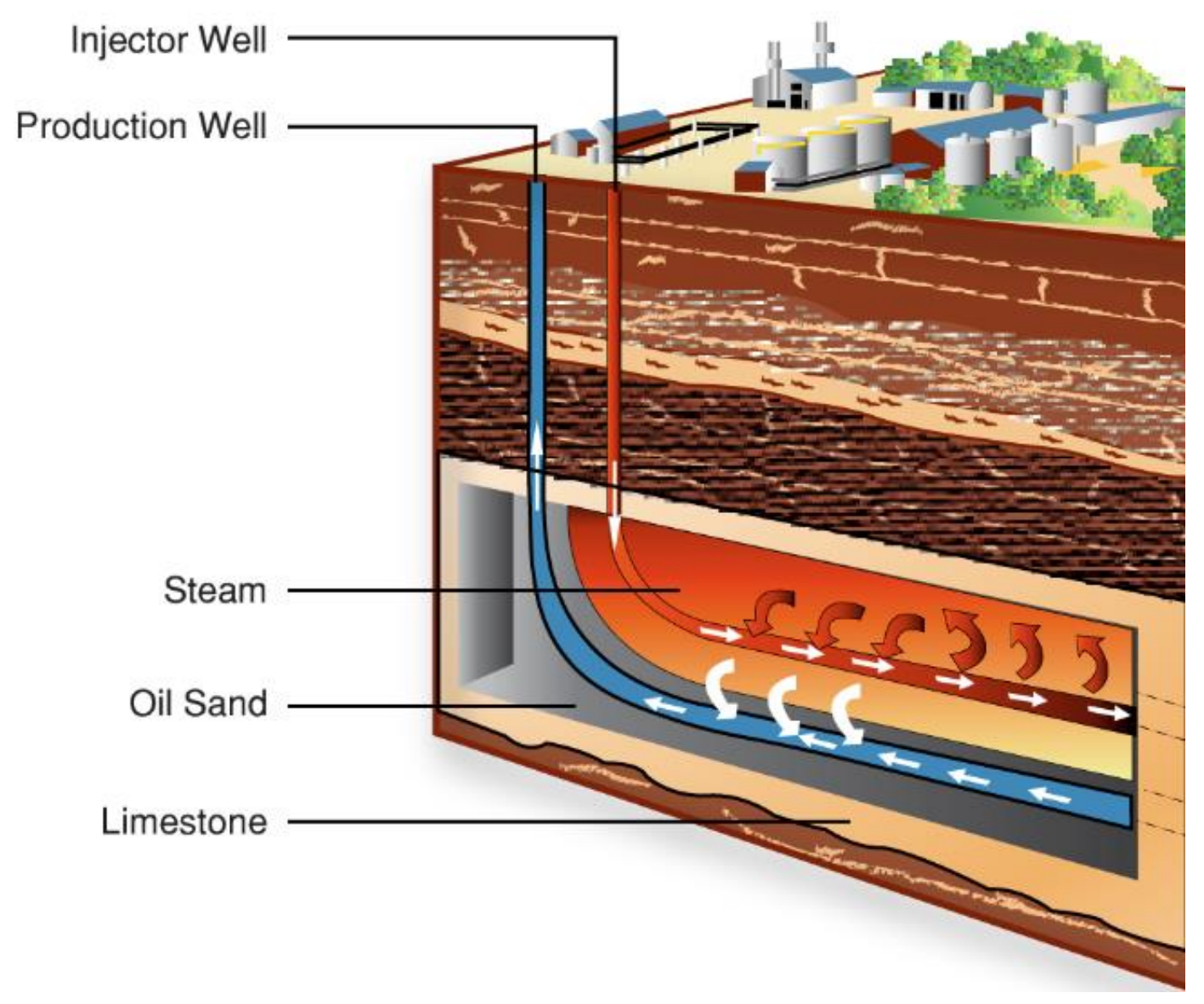

Figure 2.1 SAGD process schematic (http://jeromedowney.ca/blog/understanding-sagd) 
Two simple recovery mechanisms are involved in SAGD: viscosity reduction and gravity drainage. SAGD works best in high-quality reservoirs, more specifically reservoirs with continuous and thick net pay (part of the reservoir with oil) to accommodate two horizontal wells and with economic quantities of oil above them. A net pay of less than 15 meters is considered not economically feasible for SAGD. Another key requirement for SAGD is high vertical permeability, since gravity drainage is the driving force moving the oil and condensate to the producer. SAGD can be quite sensitive to geological heterogeneities, like inclined heterolithic strata (IHS) barriers, thief zones, etc.

\subsubsection{Cyclic Steam Stimulation (CSS)}

Besides SAGD, cyclic steam stimulation (CSS) is the most widely used thermal method of heavy oil recovery. It was first "discovered" accidentally by Shell Oil in Venezuela in 1957. A steam injector had to be shut in due to mechanical problems. After some time, the well was backflowed and produced large amounts of oil. Afterwards, cyclic steam stimulation was piloted and applied in California, in Canada, in the Middle East and around the world.

CSS consists of three main process stages (Junaid and Paul, 2007), Figure 2.2: steam injection, soaking, and production. The soaking stage is necessary to switch the well from injection to production and also to promote some condensation of steam and the heating of a larger portion of the reservoir.

During CSS, the duration of each injection and production period lengthens with time, as larger areas get drained with every cycle. In these later cycles, the peak oil rate decreases due to lower pressures and larger distances that the oil has to travel to the well. The cycle durations, rates and pressures will depend on the geology of the reservoir, oil viscosity, steam availability, pressure management strategy, lifting requirements and other factors.

The primary recovery mechanisms involved during CSS are as follows: 
- high injection pressure, which pushes sand grains apart and causes dilation in unconsolidated sand and fracturing in consolidated reservoirs;

- thermal expansion of both rock and fluids;

- viscosity reduction with heat;

- re-compaction of the rock; and

- viscous forces preventing significant hot steam vapour from breaking through the oil to the overburden.

Some of the key considerations for applicability of CSS are formation thickness, good permeability, initial oil mobility, and whether the matrix can be fractured initially.

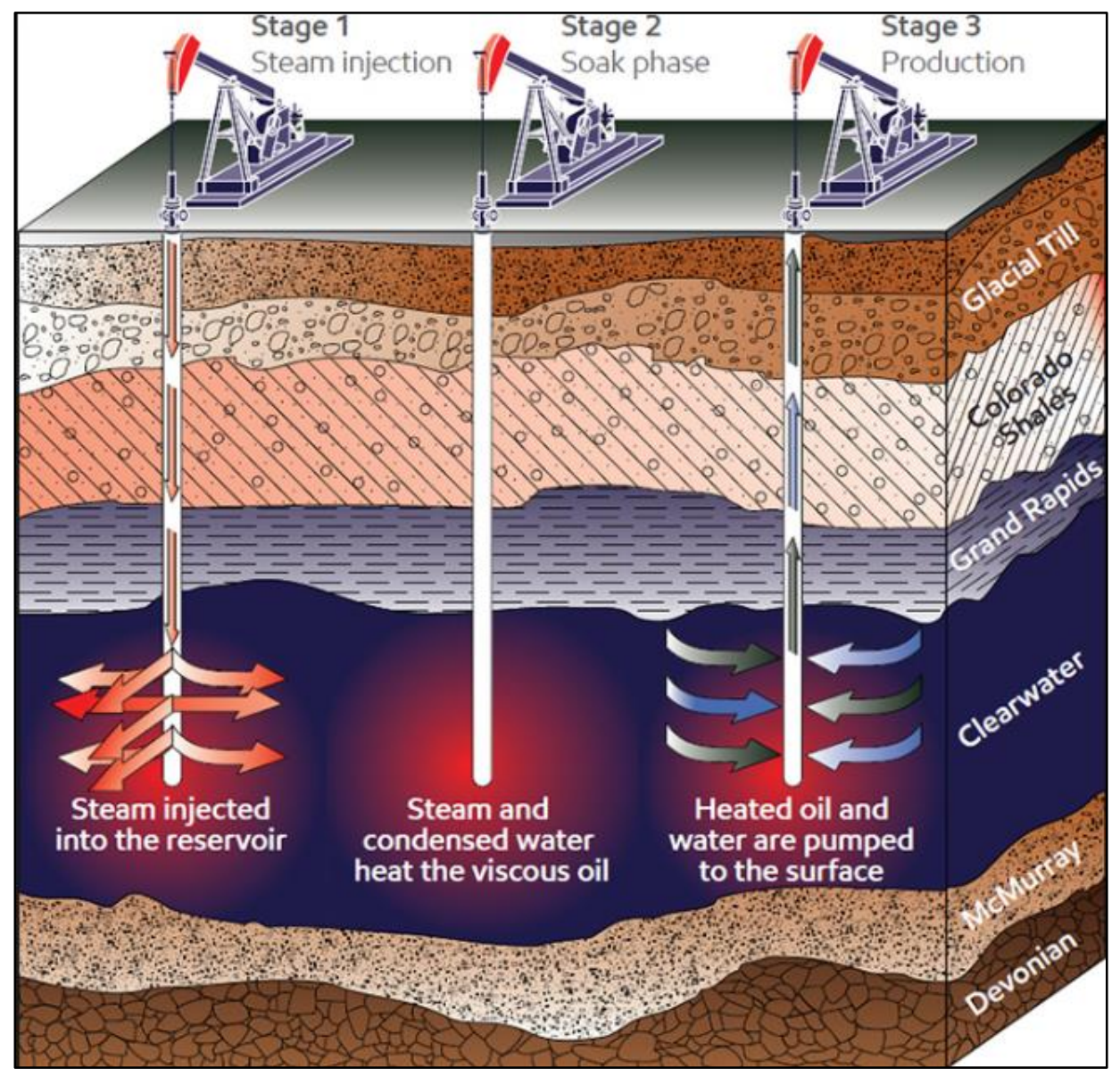

Figure 2.2 CSS process schematic (http://www.imperialoil.ca/en-ca/company/operations/oilsands/cold-lake) 


\subsubsection{Steam Flooding}

Steam flooding was first tested, even before CSS, in the 1950s. The first commercial steam floods were initiated to produce heavy oil in California. Numerous steam flood projects are operated in Indonesia, Canada, Brazil, and Venezuela. There are three distinct zones in a steam flood: steam zone, hot waterflood zone and cold waterflood zone (Chu, 1985), Figure 2.3. Different recovery mechanisms operate in each of these zones. The most important zone is the steam zone. The temperature is relatively constant in this zone once it achieves saturated steam conditions, and it contains the most mobile hot oil, which get displaced by gas drive.

Other contributing mechanisms are thermal expansion of oil (and reservoir rock) and steam distillation of the lighter components in the oil. Heating also affects the relative permeability to oil.

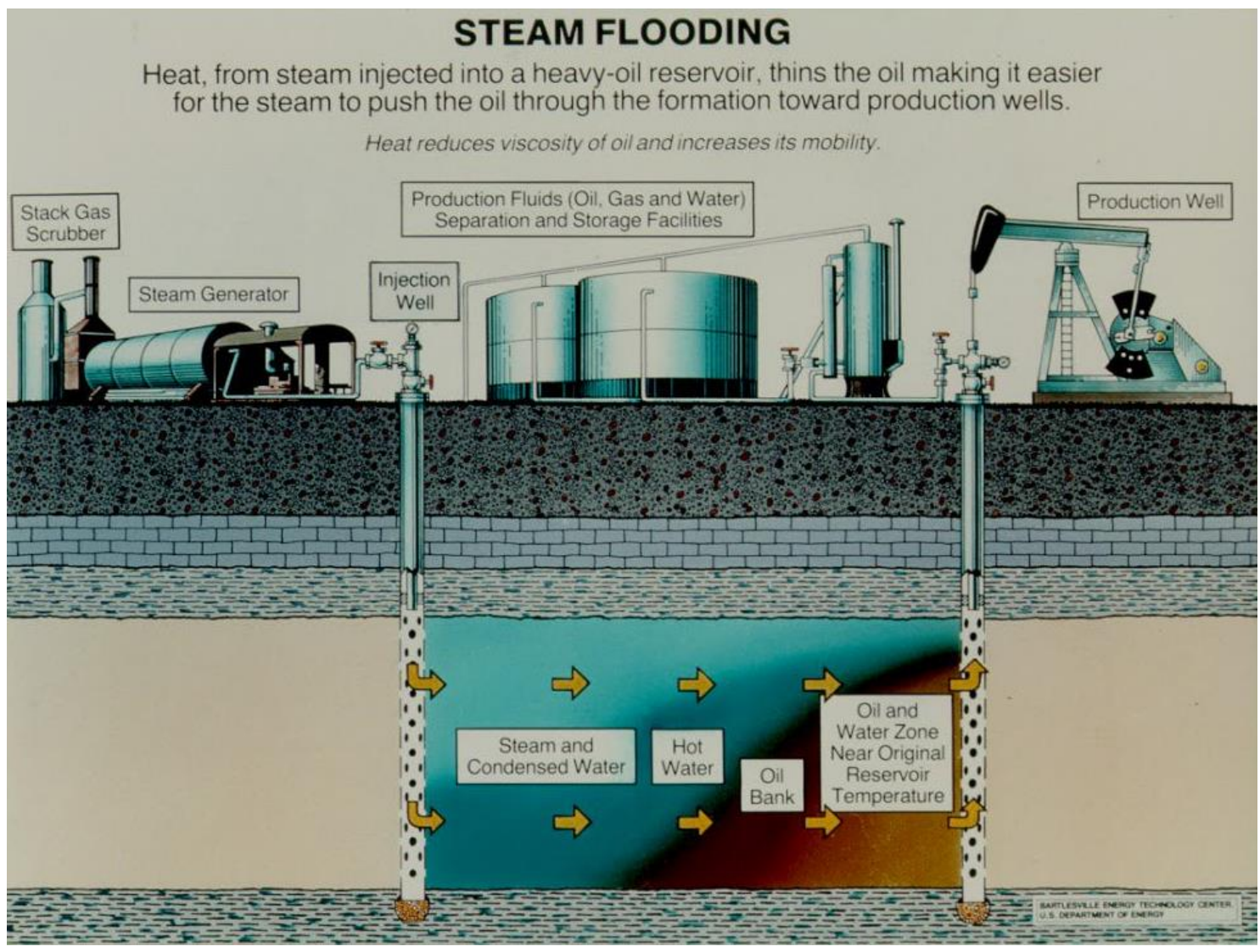

Figure 2.3 Steam flooding process schematic (http://www.steamflooding.com) 
Gravity segregation, or gravity override effect, is an important phenomenon in steam flooding. The gravity segregation of steam greatly increases sweep efficiency, but it may not be significant in reservoirs with very heavy oil or bitumen. Gravity allows steam to flow to the top of the pay zone, while hot water separates and flows beneath the steam. Gravity helps steam to spread and achieve good areal coverage of the reservoir. With time and more steam injection, the steam zone can grow downward and continue stripping hot oil from the interface. This hot oil and condensed water flow towards producing wells. The gravity segregation effect is more pronounced in reservoirs with good mobility, that is, high porosity and lower oil viscosity.

Steam flooding is somewhat more sensitive to reservoir quality. For a good steam flooding operation, initial mobility is established in the reservoir, and there is a pressure communication between injectors and producers. Good lateral permeability helps push the oil towards the producers, but fractures can channel the steam away and turn the process into a very slow imbibition-dominated process.

The best target for steam flooding is medium heavy oil - heavy enough to take advantage of high temperature and not too viscous to be immobile at reservoir conditions. As with CSS, thief zones are undesirable, although may be less detrimental to steam flooding than to CSS. Multi-layered reservoirs can reduce heat losses compared to a single thin reservoir.

\subsubsection{Electrical Heating Processes}

A more recent thermal EOR process is electricity-assisted thermal recovery. A few variations have been considered - the most straightforward method is conductive electrical heating. Resistive electrical heaters placed in wells deliver direct heat to the reservoir fluids contacting the hot heater surface. In this case, the efficiency of the process depends on effective thermal conductivity of the system, which in turn depends on the rock type (carbonate or clastic), porosity, lithology (minerals) and water content. Some of the main advantages of electrical heating are described below:

It is less sensitive to reservoir heterogeneities than steam-based processes. Heat will flow even when fluids cannot. With enough heat and expansion of immobile fluids, shales can be cracked to 
create fluid flow paths. Although electricity can be expensive, steam generation and water treatment facilities that make up a large fraction of steam project capital expenses are not required. Heater and electricity costs are operational costs that can be spread out in time, so a large amount of capital will not need to be spent on facilities before any oil is produced. Chemical reactions in rock and oil can provide some drive energy by producing gases (possibly carbon dioxide), but how much will depend on the rock, fluid and temperature.

A few processes have been developed by the industry; one such example is In Situ Reflux (Harding et al., 2016), Figure 2.4.

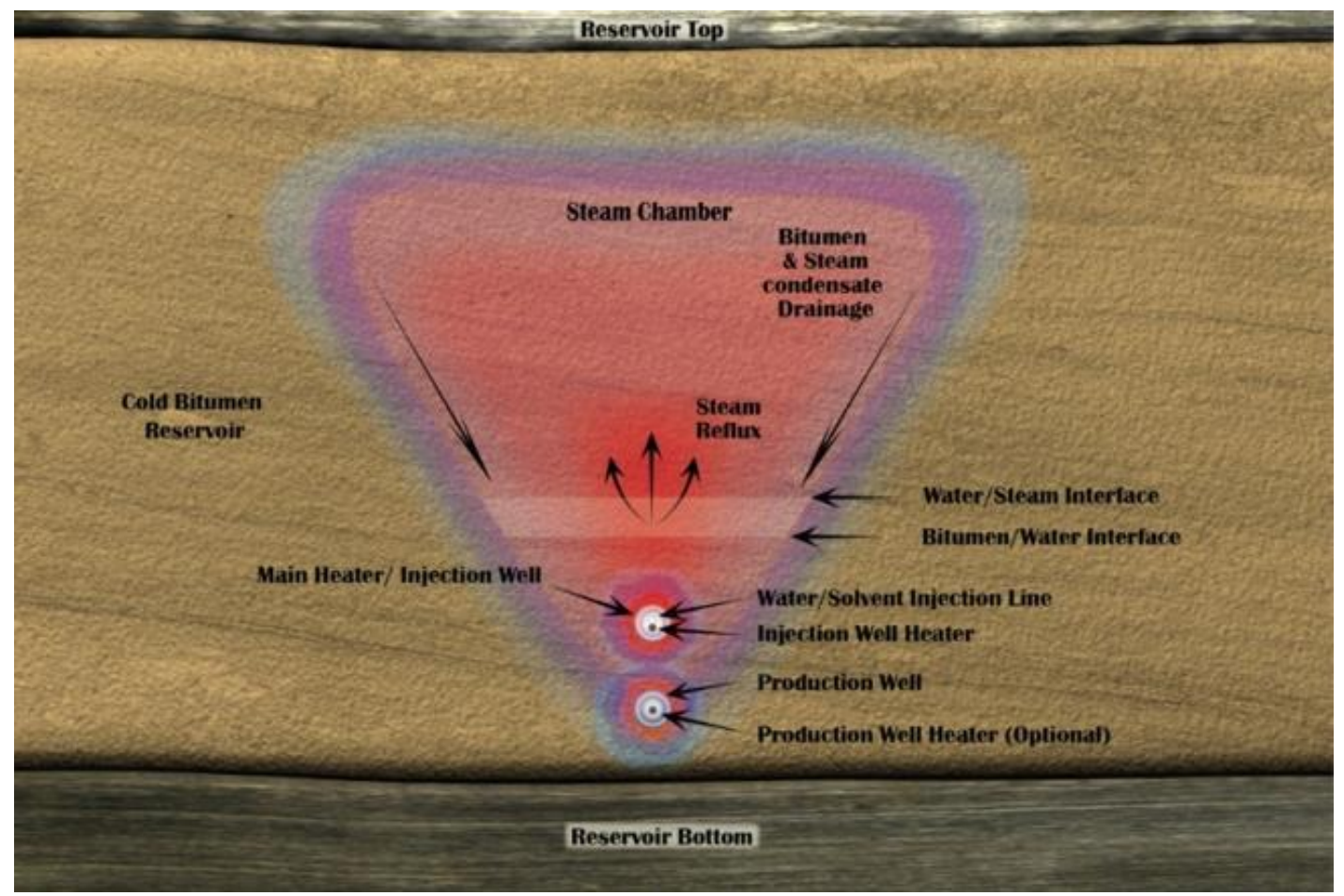

Figure 2.4 ISR process schematic (Harding et al., 2016)

The In-situ (steam) Reflux (ISR) process is an electrical EOR process with a novel, efficient and effective way to deliver energy, produced by electrical heaters, to a bitumen or heavy oil reservoir. The ISR concept involves vaporization of formation connate water and injected fluids that form a rising vapour chamber above the heater well similar to the steam chamber formed in SAGD. As the vapour rises, it loses heat to the surrounding formation matrix, connate water and bitumen, and as it cools, it condenses into liquid. This condensate and heated bitumen travel downward under 
the influence of gravity in the same manner as in SAGD. But in ISR, as these fluids approach the ISR heater well, they encounter formation temperatures above the saturated steam temperature and the water is revaporized and "refluxed" back into the steam chamber. Heated bitumen continues its downward path towards the production well, and the bitumen flow in the porous medium is uninhibited by the presence of flowing steam condensate.

The ISR process employs immersion electric resistance heaters that are placed in horizontal wells completed near the bottom of a bitumen-saturated reservoir. Both well-pair and single-well arrangements are possible. In the well-pair case, the lower well is used for production and the upper well for heating and fluid injection. Resistive electric heaters may be employed in both the upper and lower wells, and the heaters may have different power ratings and may be operated at different temperatures.

\subsubsection{Radio Frequency (RF) Heating}

Another variation of electrical heating is electromagnetic heating at radio frequencies. Heat is delivered with electromagnetic waves generated by antennas placed in the wells. The efficiency of this process depends on the impedance of the heated medium, which is directly related to the amount of in-situ water. The frequency can be altered to balance the depth of penetration vs. power or amount of heat. As with conductive electrical heating, well configurations depend on the type of reservoir being developed. Additional drive energy may also be required to produce the heated oil. Compared to ohmic resistive heating, electromagnetic heating is somewhat less efficient, because electricity has to be converted to electromagnetic waves. However, a more even and deeper reservoir heating can be achieved around the wells in less time.

One example of RF heating is ESEIEH (Effective Solvent Extraction Incorporating Electromagnetic Heating). The fundamental technology for ESEIEH (pronounced "easy") originates with Harris Corporation's method for electromagnetic heating. ESEIEH is a new in-situ oil sands recovery process that does not use steam to heat the reservoir. In ESEIEH, bitumen is concurrently heated with electrical energy and further diluted with the injection of a solvent in a gravity drainage recovery process. ESEIEH provides advantages of lower overall energy 
requirements and possible mitigation of the need for field site burning of natural gas or fossil fuels to produce steam, thereby lowering emissions (Energy Solutions Overview), Figure 2.5.

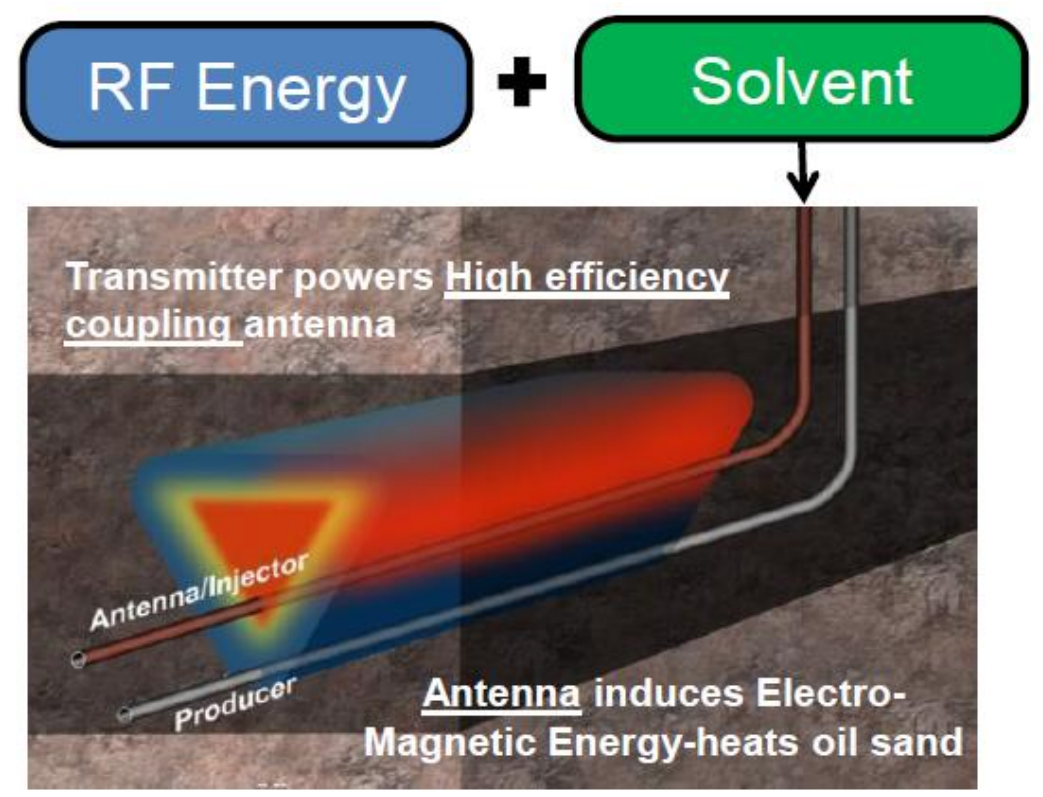

Figure 2.5 ESEIEH process schematic (Energy Solutions Overview)

\subsubsection{Steam-Surfactant Process (SSP)}

The steam-surfactant process is one in which chemicals like surfactants are added to steam. The main function of surfactants is to lower the surface tension at the interfaces between liquids or liquids and solids (Zeidani and Gupta, 2013). They may act as detergents, wetting agents, emulsifying and foaming agents or dispersants. Most surfactants are organic compounds that contain both hydrophobic and hydrophilic groups (Figure 2.6), that is, groups that, respectively, repel water and attract water. The ability of a surfactant to repel or attract water is shown with hydrophilic-lipophilic balance (HLB) in Figure 2.6. A higher value of HLB means the surfactant is more hydrophilic and a lower value of HLB means the surfactant is more hydrophobic.

To be suitable as an additive to a thermal process, the surfactants must be volatile, but stable at high temperatures. They must produce oil-in-water emulsions rather than water-in-oil emulsions, because the latter are very undesirable due to their high viscosity; they can be more viscous than the pure oil phase at the same conditions. They must be compatible with the formation, so they do 
not cause unwanted chemical reactions in-situ. They must be safe for surface facilities. Finally, the best of these surfactants should be recyclable.

The recovery mechanisms of steam-surfactant processes are not well known. Interfacial tension (IFT) reduction, wettability alteration and emulsification are all contributing factors to enhanced oil recovery_but a precise understanding is still not accomplished.

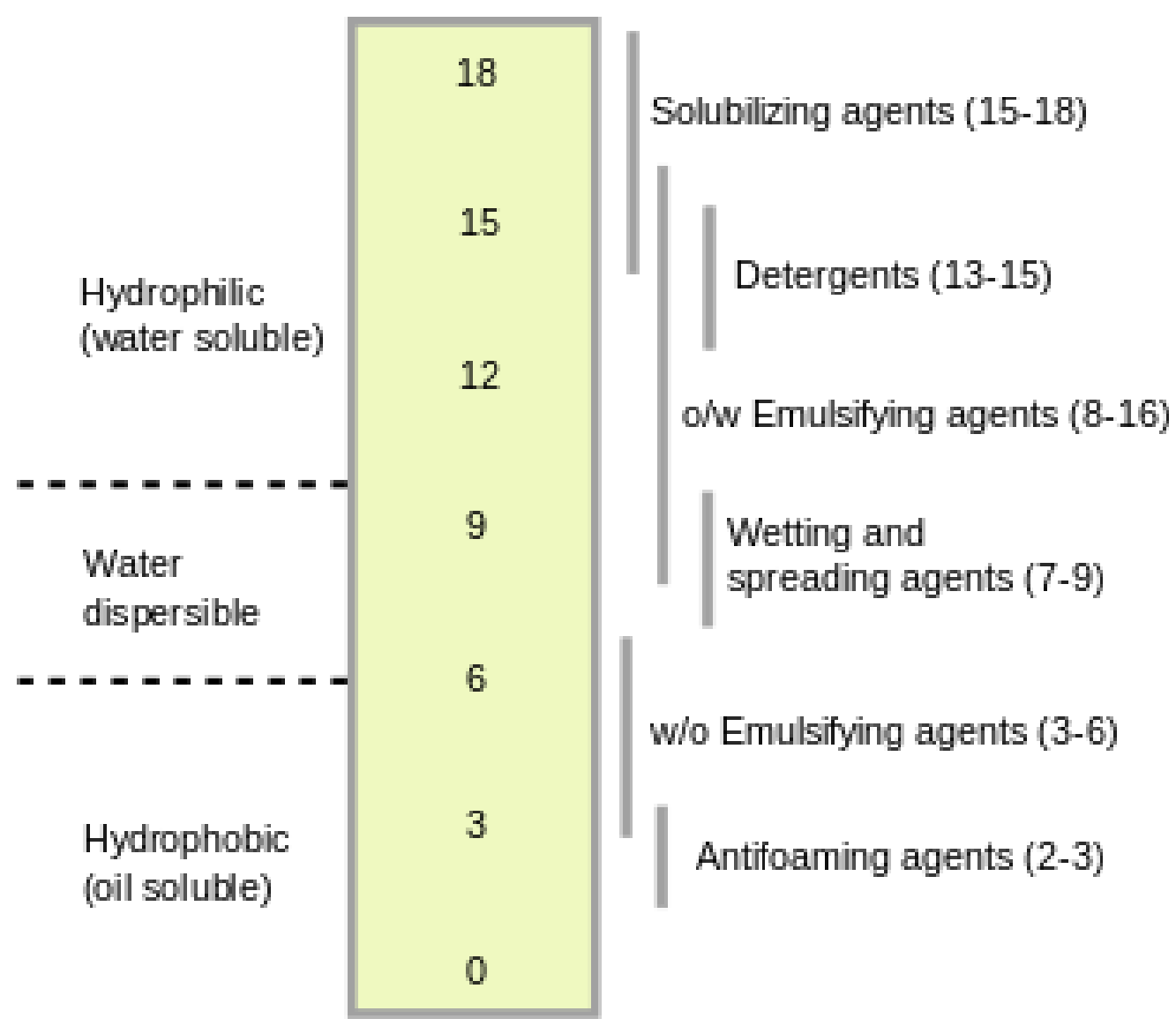

Figure 2.6 Hydrophilic-lipophilic balance of surfactants (Image @ MKD)

Selection of surfactants for thermal processes is complex because of the nature of the processes and the physio-chemical aspects of the reservoir rocks. The main complexity arises from the insitu SAGD operations that involve steam injection at elevated temperatures up to $320^{\circ} \mathrm{C}$. The biggest challenge in thermal application is the extended exposure of the surfactant to severe temperatures for longer durations that can thermally destabilize the surfactant. 
Zeidani, K. and Gupta (2013) proposed that a surfactant can be suitable for thermal application if it: can be volatile at application conditions, meaning it can vaporize at downhole SAGD operating conditions; can reduce the IFT at those conditions; is thermally stable at high temperatures; retains its properties and remains effective at high temperatures; preferentially results in oil-in-water emulsions rather than water-in-oil emulsions; enhances the reservoir wettability to water; is compatible with formation water; is readily available and at a price not so high as to make its use uneconomical; and, at downhole reservoir conditions, results in formation of stable emulsions that are easy to resolve on surface.

\subsection{Physical and Numerical Modelling of Hybrid SAGD Processes}

Hybrid SAGD is a process in which solvents are added with steam and injected into reservoirs suitable for SAGD process application. Researchers have proposed different names for hybrid SAGD depending on the type of solvent being injected and its timing of injection. Solvent-aided process (SAP), expanded-solvent ES-SAGD, and SAGD with non-condensable gases are examples of hybrid SAGD.

The solvents in combination with steam can be used in gravity-dominated, cyclic and flood-type processes. The main recovery mechanism associated with solvents is probably enhanced viscosity reduction. The physics of steam-solvent mixtures is complex. Mixtures of most hydrocarbon solvents with steam exhibit deviations from Raoult's Law for ideal mixtures (Khaledi et al., 2015), (Figure 2.7). Solvents tend to depress steam saturation temperatures and make the steam-solvent chamber cooler. Lower temperatures improve thermal efficiency, yet still achieve the same or even better viscosity reduction due to the combined effects of heat and solvent dilution.

Some additional recovery mechanisms contribute to lower residual oil saturation in the steam chamber, enhanced oil entrainment in the condensate phase due to emulsification (moderate surfactant effects at high temperatures), and in-situ deasphalting. 


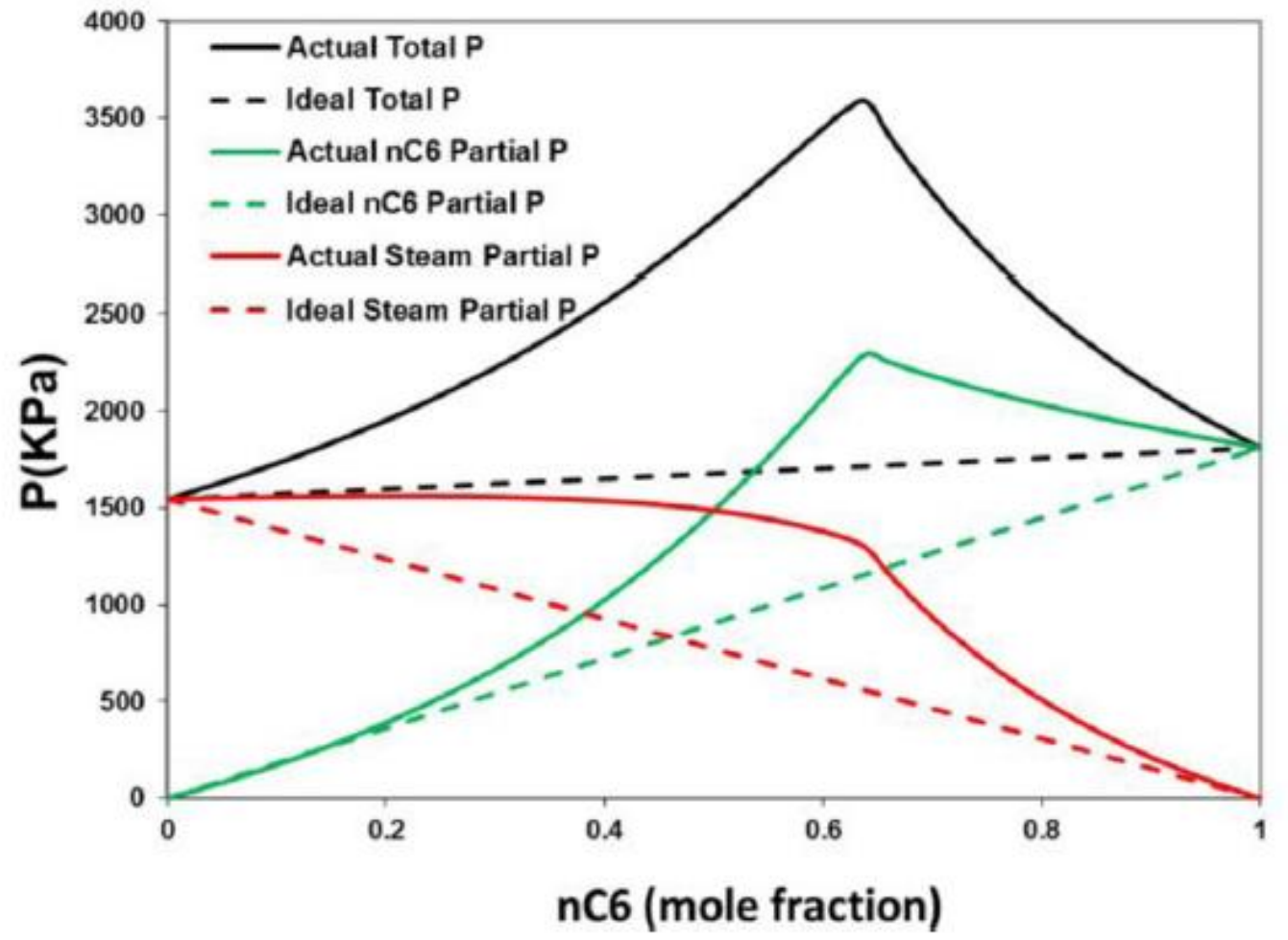

Figure 2.7 P-x diagram comparing ideal and actual partial pressure for $\mathrm{nC}_{6}$ (Khaledi et al., 2015)

Depending upon the volumes of solvent addition, the capital costs can drop along with a reduction in environmental footprint. With the use of solvents, the steam and ultimately water consumption could be less than for steam-alone processes. Furthermore, in Canada, provincial and federal governments are effectively mandating lower emissions by imposing carbon taxes and caps. With these regulations, it will be necessary to modify the thermal processes (like SAGD) to result in reduced steam consumption and a low carbon footprint. Solvent addition can be viable way to do that.

The following sections present a comprehensive survey of the literature associated with hybrid SAGD processes.

\subsubsection{Solvent-Aided Process (SAP)}

Solvent-Aided Process (SAP) is an example of a hybrid SAGD process. The fundamental concepts with respect to well configuration and recovery mechanism are the same as those of SAGD, i.e., a 
horizontal injection and production well, and viscosity reduction and gravity drainage as the primary recovery mechanisms. The concept of SAP was first introduced by Gupta et al (2003). In an SAP process, addition of a light hydrocarbon (butane) with steam can further lower the viscosity of the oil by solvent dilution (Gupta et al., 2005). Another benefit of the SAP process is that a greater well spacing can be achieved than with steam alone (Gupta and Gitiins, 2006); this affects the overall cumulative oil production because the larger spacing provides the additional oil recovery. In this process, solvent addition to the reservoir is generally begun after the steam chamber has been established. Solvent vapours travel through the steam chamber with the steam and accumulate ahead of the steam front, where they dissolve in the heated oil phase, further lowering its viscosity and thus making it more mobile. Solvent dissolution in the oil phase is beneficial; however, the excessive presence of solvent can slow down the propagation of the steam front.

The economics of the SAP process depend on the rate of oil production and the amount of solvent that is retained in the reservoir. Modelling of the SAP process has shown that the higher the amount of solvent used in SAP, the better the process performance. However, the economics of the SAP process also depend on the availability and cost of the solvent.

Gupta et al (2010) discussed using an optimal amount of solvent with steam in an SAP process. Their discussion is based on modelling that was performed to compare performance of the scheme under various solvent injection strategies. The authors used a 2D model for simulation studies with various injection strategies. The reservoir conditions and properties and injection strategies are given in Tables 2.1 and 2.2.

Their simulation results revealed that the pulsed injection of solvent in conjunction with steam leads to better performance in the SAP process, especially in terms of solvent retention in the reservoir calculated as:

Total Solvent Injected-Total Solvent Recovered

Total Oil Produced 
Table 2.1 Simulation model reservoir properties (Gupta et al., 2010)

Reservoir Dimensions $100 \mathrm{~m}$ wide x $24 \mathrm{~m}$ thick x $1 \mathrm{~m}$ long

\begin{aligned} \hline Model Grid Block Dimension & $1 \mathrm{~m}$ wide x $0.8 \mathrm{~m}$ thick x $1 \mathrm{~m}$ long \\ \cline { 2 - 2 } OOIP & $80 \%$ by volume \\ \hline Horizontal Permeability & 5.0 Darcy \\ \cline { 2 - 2 } Vertical Permeability & 4.0 Darcy \\ Oil Gravity & $8.5^{\circ} \mathrm{API} \\$\cline { 2 - 2 } Initial Reservoir Pressure & $2600 \mathrm{kPa} \\$\hline Steam Injection Rate & $900 \mathrm{~m}^{3} / \mathrm{day}$ for 1000 -meter-long injection well \\ \cline { 2 - 2 } Start of Solvent Injection & At 240 days \\ \hline Continuous Solvent Injection Rate & 0.07 tonnes/day/meter \\ \cline { 2 - 2 } Vertical Well Spacing & $4 \mathrm{~m}\end{aligned}$

Table 2.2 Butane solvent injection strategies (Gupta et al., 2010)

Constant solvent injection rate

Continuous ramp up by 1.2 times from start to end - no pulsing

Continuous ramp up by 1.4 times from start to end - no pulsing

Continuous ramp up by 2.4 times from start to end - no pulsing, 5 Darcy

30 days square pulse

30 days pulse with 2.4 times ramp up

30 days pulse with 2.4 times ramp down

Continuous ramp down by 1.4 times from start to end - no pulsing

Continuous ramp down by 2.4 times from start to end - no pulsing

The optimal solvent concentration was found to be about $10 \mathrm{wt} \%$ of the steam injection rate for both the pulsed and constant-rate solvent injection cases.

Akinboyewa et al (2010) used a compositional thermal simulator (ECLIPSE) to quantify the benefits of solvent addition to SAGD processes. The authors performed the simulations by creating a geological model from a bitumen asset in Canada. The model properties are given in Table 2.3. 
The generic schedule for solvent injection cases involved a heating period for about 100 days, a pure steam injection period for about 150 days, and commencement of a steam-solvent coinjection period.

Table 2.3 Geological model properties (Akinboyewa et al., 2010)

\begin{aligned} Model Dimensions & $150 \mathrm{~m} \times 1000 \mathrm{~m} \times 51 \mathrm{~m}(\mathrm{x}-\mathrm{y}-\mathrm{z}) \\$\hline Reservoir Depth & $323 \mathrm{~m} \\$\hline Average Thickness & $95 \mathrm{~m} \\$\hline Oil Saturation & $>75 \% \\$\hline Thickness of High Oil Saturation & $51 \mathrm{~m} \\$\hline Gridblock Division in y-Direction (Well & $49.9 \mathrm{~m} \\$ Direction) & \\ \hline Gridblock Division in x-Direction & $0.99 \mathrm{~m} \\$\hline Gridblock Division in z-Direction & $0.99 \mathrm{~m} \\$\hline Components of the Fluid Model & Bitumen, methane, butane, water \\ \hline Injected Solvent Composition & $98 \%$ butane and 2\% methane \\ \hline Reservoir Pressure & $800 \mathrm{kPa} \\$\hline Reservoir Temperature & $8.00{ }^{\circ} \mathrm{C} \\$\hline Porosity & $27.9 \% \\$\hline Horizontal Permeability & 5.70 Darcy \\ \hline Vertical Permeability & $5.30 \mathrm{Darcy} \\$\hline Initial Oil Saturation & $81.6 \%\end{aligned}$

From the field case study, the authors concluded that the higher concentration of solvent in steam requires steam volumes to be reduced. The gravity drainage resulting from steam injection becomes more efficient and produces more oil in the presence of solvent. The steam to bitumen ratio is a critical metric to evaluate the performance and efficacy of the solvent based SAGD process, in addition to the conventional steam/oil ratio (SOR). A solvent volume of 5 to 10 percent of steam's cold-water equivalent (CWE) is sufficient to produce more oil and to reduce operational costs. 
Some researchers have studied the use of propane as a light hydrocarbon additive to the steam phase (Ferguson et al., 2001). According to these studies, a preliminary start-up stage was conducted in which superheated-steam-only injection resulted in creation of a limited hot spot, especially in the proximity of the injection well. This preliminary injection procedure reduced the start-up duration of the process. Steam and propane were then co-injected, which resulted in enhanced production performance of the overall solvent-aided process as well as reduced energy requirements.

To improve and validate the reservoir simulation model and to better understanding of SAP, Sam et al (2018) carried out a history-match on an SAP pilot at Christina Lake with butane injection. The history-match was done in two steps: in the first step, the reservoir simulation model was validated by history-matching the SAGD period. Then in the second step, only the parameters and operational controls that have an impact on SAP performance were used as control variables during the SAP history-matching. The injection bottomhole pressure (BHP) was controlled in the simulation to be equal to the values observed in the field. The produced oil rates and injected steam rates were the history-matching parameters.

Among the variations in the relative permeability curves, assigning the shale barrier vertical permeability of 10 millidarcies and the rest of the reservoir with 3 darcies provided the historymatch of the SAGD production phase. The produced oil rate match was better than the injected steam rate match; however, the authors did not provide root-mean-square (RMS) between the simulated and actual data. The solvent solubility in the oil phase was modelled using the equilibrium k-values generated from the laboratory studies. The injection pressure was set as a constraint. After getting a tuned model, they ran multiple sensitivity studies and found that the produced oil rate is most impacted by injected solvent viscosity and solubility.

\subsubsection{Expanding Solvent SAGD (ES-SAGD)}

ES-SAGD, patented by Nasr (2003), Nasr and Ayodele (2005, 2006), is another example of hybrid SAGD; it co-injects a hydrocarbon additive with steam in a gravity-dominated process similar to SAGD. In this process, the solvent is injected with steam in the vapour phase. The injected solvent 
not only dilutes the heavy oil or bitumen and in conjunction with heat reduces its viscosity, but it also acts as an insulation barrier between the steam chamber and overburden, thus reducing the heat losses. More than $90 \%$ of the injected solvent is recovered with the produced fluids and about $75 \%$ of the retained solvent may be collected during the blowdown phase (ConocoPhillips, 2009).

Another name for ES-SAGD is SA-SAGD (solvent-assisted SAGD), which is an enhanced variation of SAGD process in which a small amount of hydrocarbon solvent is added to the injected steam to improve the process performance. The key idea of injecting hydrocarbon solvent with steam is that the solvent condenses along with the steam on the bitumen-vapour interface and mixes with bitumen to further reduce its viscosity and enhance the drainage rate.

ExxonMobil investigated the benefit of SA-SAGD when applied to typical Athabasca oil sands reservoir conditions (Khaledi et al., 2012). The study focused on optimization of SA-SAGD performance at relatively low pressures. The experimental reservoir model dimensions and properties were designed and scaled to represent a typical Athabasca oil sand reservoir, Table 2.4. The authors did not disclose the composition of the solvent but reported a substantial increase in the oil recovery with the addition of solvent compared to SAGD alone, Figure 2.8.

Table 2.4 Model dimensions and properties (Khaledi et al., 2012)

\begin{aligned} Dimensions & $\mathrm{L} \times \mathrm{W} \times \mathrm{H}=100 \times 60 \times 20 \mathrm{~cm} \\$\hline Porous Medium & Silica BT3 glass beads \\ \hline Porosity & 0.36 \\ \hline Permeability & $300 \mu \mathrm{m}^{2} \\$\hline Initial Oil Saturation & $88 \% \\$\hline Bitumen Viscosity & $3 \times 10^{6} \mathrm{cP}$ at $8^{\circ} \mathrm{C}\end{aligned}$




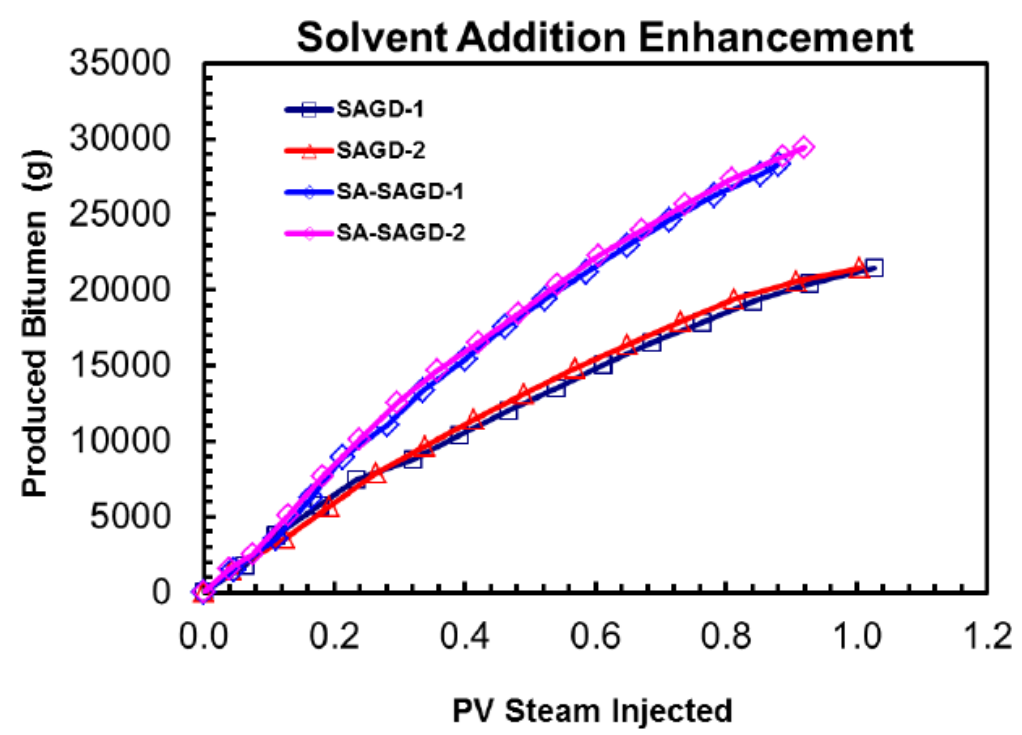

Figure 2.8 Solvent addition enhancement (Khaledi et al., 2012)

Typically, an ES-SAGD process involves co-injection of solvent and steam. ES-SAGD process improvement as compared to SAGD is mostly associated with the oil phase dilution which in turn leads to oil phase viscosity reduction. This key mechanism of enhanced oil recovery does not seem to be the most prominent one in ES-SAGD, since the solvent amounts in ES-SAGD are small, from 5-6\% by volume, which cannot reduce the oil viscosity to the extent where enhanced oil recovery from 25 to $100 \%$ can be achieved. Ezeuko et al (2012) directed the attention to another important aspect of ES-SAGD, i.e., emulsification at the steam chamber edge. The authors presented a numerical approach that allows the incorporation of emulsion modelling into SAGD and ES-SAGD.

Emulsion is a colloidal system of immiscible fluids, with one fluid as the dispersed phase while the other is the continuous phase. These emulsions are stabilized by surface active agents that absorb at the oil/water interface. Natural emulsifiers such as asphaltenes are common in heavy oils and bitumen (Zhao et al., 2009). According to Bancroft's (1913) rule, water in oil (WO) emulsion is most likely to form when oil and water mix in the presence of asphaltenes. Experimental studies showed that emulsion produced during SAGD is predominantly a water in oil type emulsion (Noik et al., 2005). 
Jiang et al (2012) studied the solvent injection strategy in the ES-SAGD process by conducting a detailed numerical modelling study. The authors incorporated the measured data on key physical properties into the numerical model and validated it by history-matching the laboratory experimental data with $\mathrm{n}$-hexane as a solvent. Then they used the numerical model to evaluate the ES-SAGD performance with different operating strategies. The experiments were performed using a 2D high pressure/high temperature cell at Alberta Innovates Technology Futures (AITF). The initial model conditions are listed in Table 2.5.

Table 2.5 Model dimensions and properties (Jiang et al., 2012)

\begin{aligned} Dimensions & $\mathrm{L} \times \mathrm{W} \times \mathrm{H}=10 \times 80 \times 24 \mathrm{~cm} \\$\hline Porous Medium & Sand \\ \hline Porosity & $31.7 \% \\$\hline Permeability & 120 Darcy \\ \hline Initial Oil Saturation & $88.0 \% \\$\hline Bitumen & Athabasca \\ \hline Steam Injection Rate, gm/min & 32.6 \\ \hline Solvent Injection Rate, gm/min & 3.30 \\ \hline Solvent wt\% & 9.50\end{aligned}

The model designed by Jiang et al (2012) was equipped with a grid of thermocouples with injection and production wells placed in the centre middle along the width, Figure 2.9. After the model was pre-heated, both the solvent and steam were co-injected through the injection well at a temperature of $220^{\circ} \mathrm{C}$ and pressure of $2100 \mathrm{kPa}$, gauge.

For the ES-SAGD process, Jiang et al (2012) incorporated properly measured k-values for the solvent into the numerical model; otherwise, it is not an easy task to numerically history-match the solvent production behaviour. In the modelling study, the authors suggested to start the coinjection of steam-hexane earlier to be beneficial to the oil rate increase for the ES-SAGD process. This oil rate lifting becomes less effective later in ES-SAGD. Instead of continuous steam-solvent injection, stopping the steam-solvent injection at certain points appear to be favourable to reducing solvent usage and enhancing solvent recovery while maintaining good oil production. The ES- 
SAGD process performance can be improved by adjusting solvent concentration in the steamsolvent injectant and the duration of steam-solvent co-injection.

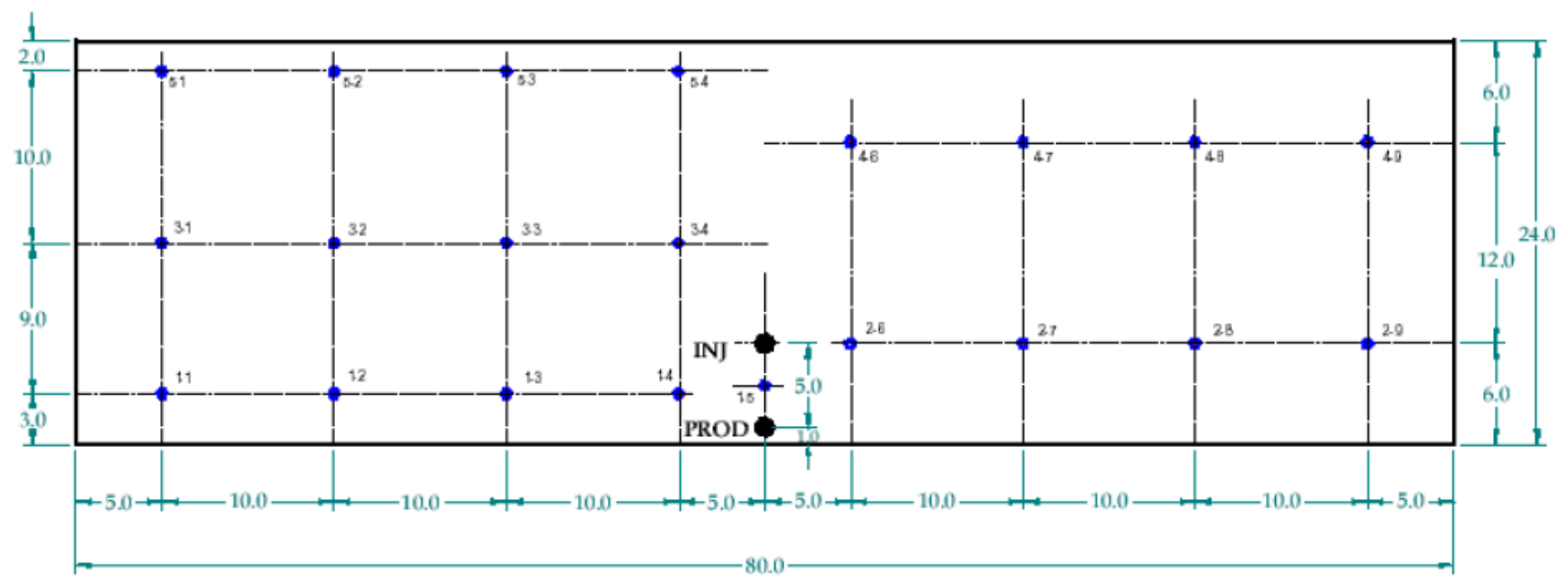

Figure 2.9 Physical model thermocouple grid (Jiang et al., 2012)

In order to select the solvent for a typical ES-SAGD process, Nasr et al (2003) proposed that the vaporization temperature of the solvent should match that of the steam phase at operating pressure and that the hydrocarbon steam additive should remain in the gaseous state when migrating from the injection spot until it reaches the bitumen surface. Nasr et al (2003) showed that as the condensable hydrocarbon solvent achieves heavier molecular weight, the resulting recovery performance would be better; however, there is a local maximum in the production performance curve of the ES-SAGD process.

Gates and Chakrabarty (2008) designed a solvent injection strategy for a single-well-pair ESSAGD process by optimizing the ratio of net energy injected to oil in a detailed and realistic 3D model of a heavy oil reservoir. The process parameters included the injection pressure and relative amounts of steam and solvent in the injected stream. The reservoir model was heterogeneous with respect to porosity. The reservoir simulator input parameters are shown in Table 2.6.

The production well was located 3 meters above the bottom of the reservoir pay. The injection well was located 5 meters above the production well. The length of the wells was 750 meters. At 
the injection well, the steam injection pressure was constrained to a maximum bottomhole pressure which was set by the optimization algorithm. The steam quality at the sand face was 0.8 . The reservoir simulations were performed by initially putting the injection and production wells on steam circulations modelled as line heaters for three months. Hexane was used as a surrogate solvent for diluent. Gates and Chakrabarty (2008) found that the solvent addition to SAGD can yield lower cumulative energy to oil ratios than SAGD and therefore can be energetically more efficient than SAGD.

Table 2.6 Numerical simulator input parameters (Gates and Chakrabarty, 2008)

\begin{tabular}{|c|c|}
\hline Initial Reservoir Temperature $\left({ }^{\circ} \mathrm{C}\right)$ & 10.00 \\
\hline Initial Reservoir Pressure at Reservoir Depth, $\mathrm{kPa}$ & 1,210 \\
\hline$K_{v} / k_{h}$ & 0.200 \\
\hline$S_{\text {orw }}$ & 0.200 \\
\hline$S_{w c}$ & 0.150 \\
\hline$S_{\text {org }}$ & 0.005 \\
\hline$S_{g c}$ & 0.050 \\
\hline$K_{\text {rwro }}$ & 0.100 \\
\hline$k_{\text {rocw }}$ & 0.992 \\
\hline$k_{\text {rogc }}$ & 0.834 \\
\hline$K_{\text {rg(sorg) }}$ & 1.000 \\
\hline Rock and Overburden Heat Capacity, $\mathrm{KJ} / \mathrm{m}^{3}{ }^{\circ} \mathrm{C}$ & 2,600 \\
\hline Rock and Overburden Thermal Conductivity, $K J / m$ day ${ }^{\circ} \mathrm{C}$ & 660 \\
\hline Bitumen Thermal Conductivity, $\mathrm{KJ} / \mathrm{m}$ day ${ }^{\circ} \mathrm{C}$ & 11.50 \\
\hline \multirow[t]{3}{*}{ Bitumen Viscosity Correlation } & $\ln \ln \mu(\mathrm{cp})=\mathrm{A}+\mathrm{B} \ln T(\mathrm{k})$ \\
\hline & $A=22.85$ \\
\hline & $\mathrm{B}=-3.578$ \\
\hline Solution GOR, $\mathrm{m}^{3} / \mathrm{m}^{3}$ & 3 \\
\hline
\end{tabular}

Ivory et al (2007) performed experiments and numerical simulations to determine the effectiveness of the thermal solvent reflux and thermal solvent hybrid processes. The technical objective of the thermal solvent reflux experiments was to develop a hybrid SAGD process for recovery of heavy oil from thick, cold reservoirs such as Cold Lake and Athabasca. The authors conceptualize the 
thermal solvent process as follows: inject solvent and produce oil through horizontal wells, heat the injection and production wells to re-boil the solvent in-situ, and in-situ recycle the solvent. The concept for thermal solvent hybrid process was to deliver heat through co-injected steam.

Ivory et al (2007) adopted the scaling approach developed by Pujol and Boberg (1972) to design their experiments. A scaling factor of 100 was chosen, which resulted in following scaling relationships:

Length $_{\text {Field }}=100 \times$ Length $_{\text {Lab }} ; \quad$ Volume $_{\text {Field }}=100^{3} \times$ Volume $_{\text {Lab }} ; \quad$ Time $_{\text {Field }}=100^{2} \times$ Time $_{\text {Lab }}$; Rate $_{\text {Field }}=100 \times$ Rate $_{\text {Lab }}$; and PermeabilityField $=100 \times$ PermeabilityLab

The scaling method developed by Pujol and Boberg (1972) has the following requirements:

$\checkmark$ The field and model must be geometrically similar, i.e., width to length ratio and height to length ratio must be the same.

$\checkmark$ The physical and thermal properties of fluid and rock (some of them such as: fluid density and viscosity) must be the same.

$\checkmark$ The field and the lab must have the same initial temperature and pressure conditions.

The aforementioned scaling approach captures the gravity forces, viscous forces and diffusion but does not capture the viscous fingering, capillary forces and dispersion.

A total of four experiments were performed with the different model dimensions shown in Table 2.7 .

The model was packed with Ottawa sand and was insulated for heat loss and confined by nitrogen overburden. Oil from Hillmond field (operated by Husky Energy) with viscosity of 27,240 cP at $20^{\circ} \mathrm{C}$ and Athabasca UTF bitumen with viscosity of $47,000 \mathrm{cP}$ at $30^{\circ} \mathrm{C}$ was used in these experiments. During all these experiments, 99\% pure propane was used as solvent additive. 
Table 2.7 3D physical model dimensions (Ivory et al., 2007)

\section{Experiment 1 (Model Symmetry) - Hillmond Oil - $\mathrm{L} \times \mathrm{W} \times \mathrm{H}=45 \times 12.5 \times 20 \mathrm{~cm}^{3}$ \\ Solvent Mixed with Steam \\ Experiment 2, 3 and $4 \mathrm{~L} \times \mathrm{W} \times \mathrm{H}=90 \times 10 \times 30 \mathrm{~cm}^{3}$}

\section{In addition for Experiment 4: Wells Heated by}

Injected Steam

The experimental results from four experiments were history-matched using CMG's STARS simulator. The total dispersion of the solvent was used to simulate both diffusion and dispersion, represented by the following equation:

$$
\begin{aligned}
& J_{i j k}=\frac{-\left(\varphi \mathrm{S}_{j} D_{i j}\right)}{F_{j k}} \frac{\partial c_{i j}}{\partial k} \\
& D_{i j}=\frac{D_{i j}^{*}}{F_{j k}}+\alpha_{j k} \mathrm{u}_{j k}
\end{aligned}
$$

where, $J_{i j}$ is flux of component $i$ in phase $j$ in $k$ direction, moles $/ \mathrm{m}^{2} / \mathrm{sec}, \phi$ is porosity, $S_{j}$ is saturation of phase $j, D_{i j}$ is molecular diffusion, $\mathrm{m}^{2} / \mathrm{s}, c_{i j}$ is solvent concentration of component $i$ in phase $j, F_{j k}$ is tortuosity experienced by phase $j$ in $k$ direction, $D_{i j}^{*}$ is molecular diffusion of component $i$ in phase $j, \mathrm{~m}^{2} / \mathrm{s}, \alpha_{j k}$ is mechanical dispersivity for phase $j$ in direction $k, \mathrm{~m}^{2} / \mathrm{s}$ and $\mathrm{u}_{j k}$ is interstitial pore velocity of phase $j$ in direction $k$.

Ivory et al (2007) found that with the non-thermal Vapex process, the value of the dispersion/diffusion coefficients has a profound effect on predicted oil and gas production in the thermal solvent processes. The decision for what values to use for thermal processes is more difficult as they are highly dependent on temperature as well as on concentration. Additionally, both the steam and solvent play a major role in improving the oil production from thermal solvent processes.

Simangunsong (2006) conducted experimental, analytical and simulation studies to investigate the use of propane and petroleum distillate as steam additives to enhance production of a $12^{\circ} \mathrm{API}$ and 
$2800 \mathrm{mPa} \cdot \mathrm{s}$ heavy oil. The experiments consisted of injecting pure steam, steam-propane, and steam-petroleum distillate into a vertical cell $(65.85 \mathrm{~cm}$ long and $7.376 \mathrm{~cm}$ diameter) containing a mixture of sand, water and oil at the reservoir temperature of $53^{\circ} \mathrm{C}$. Superheated steam at $230^{\circ} \mathrm{C}$ was injected at $5.5 \mathrm{~mL} / \mathrm{min} \mathrm{CWE}$ simultaneously with propane or petroleum distillate while the outlet pressure was maintained at 260 psi gauge. The experimental results indicated that, compared to pure steam injection, oil production was accelerated by 30\% for 5:100 propane:steam injection and 38\% for 5:100 petroleum distillate:steam (both weight ratios) injection, respectively. The petroleum distillate and propane as additives improved the injectivity of the fluids by reducing the pressure differential to $150 \%$ and $240 \%$. The steam front was faster in the steam-propane runs, which resulted in an earlier start of oil production during solvent-added runs.

King et al (2005) showed that the effect of the solvent dilution on oil viscosity is less prominent as the temperature increases. The bitumen sample used for the study was sales oil from PetroCanada's Mackay River SAGD operation, having a density of $1006 \mathrm{~kg} / \mathrm{m}^{3}$ at $15.6^{\circ} \mathrm{C}$ and viscosity of $356,800 \mathrm{mPa} \cdot \mathrm{s}$ at $20^{\circ} \mathrm{C}$. Three different solvents of different carbon number distribution were used. The aliphatic volume per cent of $\mathrm{C}_{1}-\mathrm{C}_{4}$ and $\mathrm{C}_{5}-\mathrm{C}_{30+}$ in these three solvents was: 0 and $78.1 \%$, 12.3 and $66.8 \%$, and 8.4 and $63.6 \%$, respectively. King et al (2005) used a capillary viscometer to measure the viscosity of the diluted oil at different temperatures. The viscosity was calculated from the measured pressured drop across the capillary tube using the Hagen-Poiseuille relationship.

\subsubsection{SAGD with Non-Condensable Gases}

SAGD and its variants have become the dominant technology to recover heavy oil and bitumen in Canada. SAGD can become more complicated in the presence of gases like methane, nitrogen, air etc., which can significantly affect the displacement of oil. Non-condensable gases (NCG) are gases which can be present in the SAGD steam chamber but do not dissolve into the liquid phase to any large extent at steam temperatures (Aherne and Birrell, 2002).

Butler (1997) and Butler and Jiang (2000) developed a process called SAGP (Steam and Gas Push) by employing methane to improve the efficiency of the SAGD process. SAGP is operated at lower 
temperatures than SAGD; thus, lower amounts of heat are lost to the overburden and to the reservoir rock.

Ito et al (2001) performed a simulation study on the effect of gas injection on the SAGD process. The study revealed that co-injection of non-condensable gas negatively impacts the growth of the steam chamber due to accumulation of this gas on the leading edge of the steam chamber. The authors demonstrated that the addition of non-condensable gas can cause a decrease in ultimate oil recovery at an early production stage with higher steam to oil ratios. However, the numerical study was not validated with actual data.

Al Murayri et al (2011) developed a simulation model and showed that non-condensable gas addition reduces the total amount of injected steam compared to SAGD and results in lower cumulative steam to oil ratios.

Ardali et al (2012) performed an extensive simulation study to understand the mechanism of noncondensable solvent addition to the SAGD process when initial solution gas is present. The results show a negative effect on the oil recovery in the presence of initial solution gas in the Athabasca reservoir.

Gases such as carbon dioxide, nitrogen, hydrogen sulfide and methane can be present in the steam chamber during SAGD but do not condense into a liquid phase to any large degree. The literature suggests that, theoretically, these gases can enhance the thermal efficiency of SAGD without a significant reduction in thermal conductivity. Li et al (2012) did an extensive numerical model study to analyze the impact of non-condensable gases in SAGD. The simulation suggested that, during a SAGD operation in the presence of NCG, these gases tend to rise to the top of the reservoir and provide a thermal and pressure insulation effect that limits the rate of front spreading at the corners of the chamber.

Hossein et al (2015) conducted a full field-scale simulation and attempted to provide insight into the effect of injecting non-condensable gas in the hybrid SAGD process. They considered important mechanisms such as diffusion and dispersion of gases and solubility of these gases in 
the liquid phase. The authors developed a two-dimensional heterogenous model in CMG's STARS simulator, measuring $100 \mathrm{~m} \times 25 \mathrm{~m} \times 30 \mathrm{~m}$. The gridblock size was $1 \mathrm{~m}$ in the I (width) and $\mathrm{K}$ (height) directions and $25 \mathrm{~m}$ in the $\mathrm{J}$ (length) direction. A pressure/volume/temperature (PVT) model was built based on the available literature data for the density and viscosity of the raw bitumen. The two wells - injection and production - were defined 5 meters apart in the centre of the model and were operated at a constant pressure constraint. The injection pressure was fixed at $2200 \mathrm{kPa}$ and steam was injected at $215^{\circ} \mathrm{C}$ at $80 \%$ steam quality. The authors considered ratedependent dissolution and ex-solution of gases in the liquid phase. The results indicated an improvement in the oil recovery with reduction in the SOR when methane was co-injected with steam with a mole percentage of less than $3.0 \%$. The simulation study was not validated against physical data.

\subsubsection{Hybrid SAGD - Some Field Examples}

There are a few projects in Canada in which solvents were used to enhance thermal recovery. Encana Corporation has been piloting its solvent-aided process at the Senlac, Saskatchewan, SAGD (Gupta et al., 2005) and Christina Lake, Alberta, SAGD (Gupta and Gittins, 2006) projects. The operators used butane with steam at 17 mass $\%$ and reported good results from a technical point of view. The bitumen production (or recovery) rates were reported to be up from 50 to $150 \%$. The steam to oil ratios (SOR) were lowered from 5 to $1.6 \mathrm{sm}^{3} / \mathrm{sm}^{3}$ at the Christina Lake project. The partial upgrading of in-situ oil by de-asphalting was reported to be improved by $1^{\circ} \mathrm{API}$. Solvent recovery of about $70 \%$ was also reported.

Connacher Oil and Gas has been using some light hydrocarbon solvent (which the company has not disclosed) at its SAGD project in Algar, Alberta (AER Presentation). A liquid-vapour equilibrium (LVE) of 10 to $15 \%$ was employed with steam. It was reported that the bitumen recoveries were up by $28 \%$, SOR was reduced by $16 \%$ and solvent recovery of up to $89 \%$ was achieved.

Imperial Oil has been developing its LASER (liquid addition of solvent to enhance recovery) process for many years now. The first pilot was done in Cold Lake, Alberta, in 2002 (Leaute, 
2002). A commercial project with 240 wells was started in Cold Lake in 2007 (Stark, 2013). The LASER process involves adding commercially available diluent (mostly heptane) to the later cycles in cyclic steam stimulation at Cold Lake. The oil to steam ratios were reported to be up by an average of $50 \%$, and 50 to $70 \%$ of the diluent was recovered.

Shell Canada used gas condensate solvent to enhance a steam flood that followed CSS recovery in Peace River, Alberta (Castellanos et al., 2016). A gas condensate slug of $15 \mathrm{wt} \%$ was used in a steam flood in a post-CSS reservoir. Enhanced production of bitumen of $0.49 \mathrm{sm}^{3} / \mathrm{sm}^{3}$ of solvent, and solvent recovery of about $60 \%$ were reported.

Imperial Oil piloted the addition of gas condensate to its SAGD operations in Cold Lake (Zeidani and Gupta, 2013). The operators reported some very encouraging improvements in bitumen production rates and SORs. The bitumen rate was doubled and SOR was down by $35 \%$. However, solvent recovery in the field was unclear.

ConocoPhillips has been running a solvent pilot in two well pairs in its Surmont SAGD project in Alberta as a joint venture with Total (AER presentation, 2014). Bitumen production rates were up by 26 to $36 \%$, SOR was down by 14 to $27 \%$, water cut was down by 5 to $6 \%$ and solvent recovery ranged from 33 to $39 \%$.

\subsection{Scope for Further Development of Hybrid SAGD}

As reported in the literature cited above, hybrid thermal processes have the technical potential to improve thermal EOR process efficiency. Most of the findings associated with hybrid SAGD performance are based on numerical simulation studies, most of which lack validation of the numerical models against laboratory or field data and probably incorrectly predict the solvent behaviour in the steam chamber and its impact on hybrid SAGD performance. Lack of experimental data is another issue related to simulation of this behaviour. A direct comparison of using condensable and more soluble gases like propane as opposed to non-condensable and less soluble gases like methane or nitrogen also seem to be missing from the literature. Moreover, the effect of gaseous solvent concentration in the injected stream has not been investigated much. 
The current hybrid application seems to be more focused on reservoirs suitable for SAGD, i.e., those with thick pays and those where the overburden is thick enough to allow high-temperature steam injection at $250^{\circ} \mathrm{C}$ or above. Not much work is reported for application of hybrid thermal processes to the massive heavy oil resources - approximately over 600 billion barrels - that are too shallow to mine and too deep to apply conventional SAGD.

For a full-scale commercialization of thermal hybrid EOR processes, on technical grounds alone, economic feasibility is the challenging question. In an economic evaluation, there are many variables and uncertainties to consider. Some of the most important ones are: cost of solvent, its recyclability and retention in the reservoir, and environmental issues. As indicated throughout the literature survey, the hydrocarbon solvents are good candidates as an additive to thermal EOR, especially for SAGD; however; the price tag for some solvents is even higher than the price tag for the oil that is being recovered. In this case, the option of co-injecting non-condensable gases with steam seems not illogical for the techno-economical improvement of the SAGD process.

The opportunities for hybrid SAGD processes need further exploration in terms of their application in low-pressure reservoirs. Nexen Inc.'s Long Lake bitumen field in Alberta is such an example where the reservoir pressure is 1.3 to $1.5 \mathrm{MPa}$.

The performance of hybrid SAGD using solvents that are readily available, for example noncondensable solvents like methane, carbon dioxide and nitrogen, needs to be compared to that using condensable hydrocarbon solvents that are not readily available but are more soluble in oil, for example propane. Proper solvent concentration is also an important factor that needs to be investigated for best hybrid SAGD process performances. Furthermore, the effect of solvent addition, especially of non-condensable gases, in simulations of SAGD needs more effort to accurately model this behaviour and validate it against the actual data. 


\subsection{Objectives of Current Work}

The objectives of this study are as follows:

1. Develop a laboratory-scale experimental setup to conduct hybrid SAGD experiments with the placement of horizontal injection and production wells.

2. Inject gaseous solvents - nitrogen, carbon dioxide, methane and propane (with different concentrations of 5.0, 7.5 and $10.0 \mathrm{wt} \%$ ) —with steam, and compare the performance of the resultant hybrid SAGD process with that of a steam-alone process. Note that as indicated in the literature, solvent concentration for hybrid SAGD process can vary from 5 to $10 \mathrm{wt} \%$. Therefore, to cover the whole range, three concentrations of 5.0, 7.5 and 10.0 wt $\%$ were selected.

3. Identify and evaluate the best solvent additive for a hybrid SAGD process when applied in low-pressure reservoirs.

4. Investigate the effect of gas concentration on the hybrid SAGD performance.

5. Conduct numerical modelling of the hybrid SAGD process, and validate it experimentally.

\subsection{Structure of the Dissertation}

This dissertation is organized into seven chapters.

Chapter 1 introduces crude oil and its different resources around the world. State-of-the-art oil recovery technologies are also presented. 
Chapter 2 provides a literature review on evaluation of hybrid SAGD processes, including laboratory-scale studies, numerical simulations and field examples. The scope for further development, and related objectives of the current study, are also outlined.

Chapter 3 provides the details of the experimental study that is the focus of this dissertation, including experimental methods, materials used, and error analysis of the experimental data. Several experiments were performed in which the type of the solvent and its concentration in a hybrid SAGD process were varied. Data were collected for dead oil density and viscosity, for porous matrix permeability and porosity, and for oil recovery.

Chapter 4 provides the experimental results and discussions in terms of evaluating the effect of gaseous solvent concentration, as well as the effect of different gases on hybrid SAGD process performance.

Chapter 5 provides the methodology to develop the numerical model used to history-match the experimental data. The fundamental equations, along with important assumptions and initial and boundary conditions, are presented in this chapter.

Chapter 6 provides the history-matched results of solvents that were found to be promising. The tuned relative permeability curves for each concentration of carbon dioxide, nitrogen and methane are also presented in this chapter.

Chapter 7 summarizes the contributions of this research. Suggestions for future work and recommendations are presented in this final chapter. 


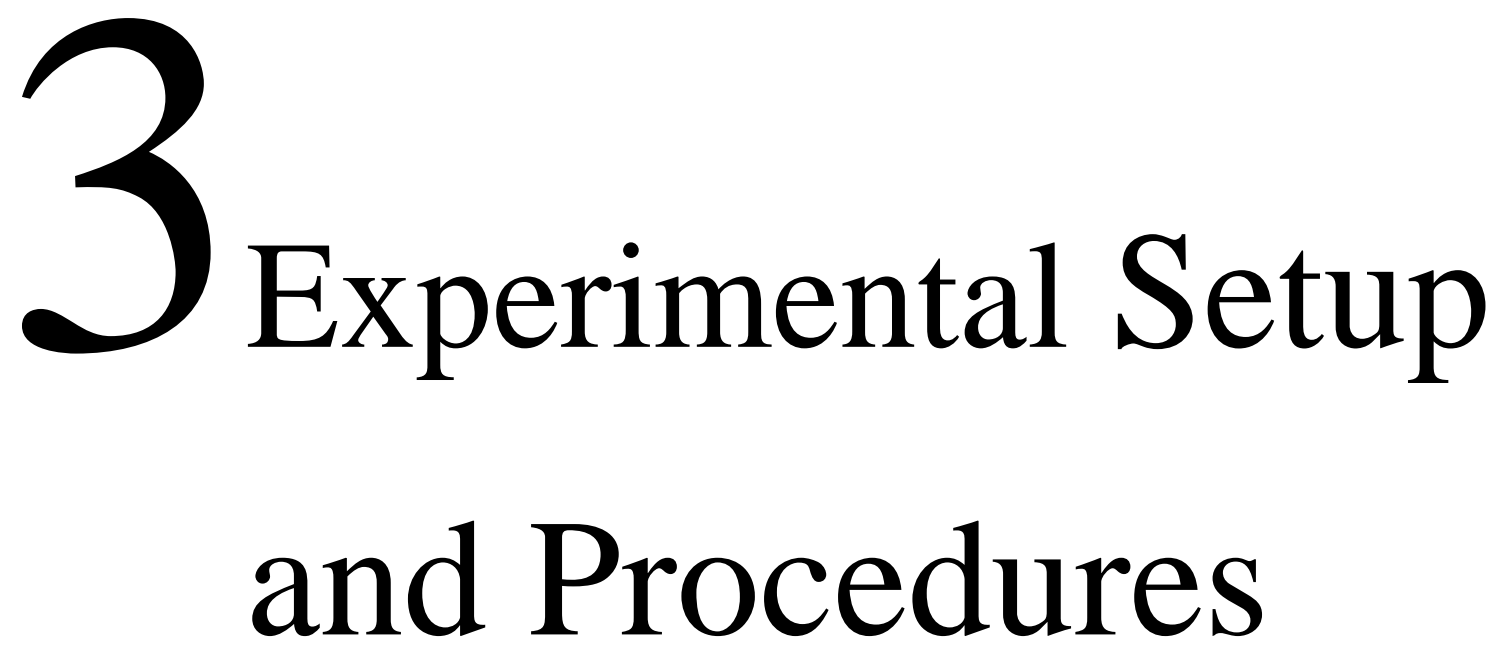

This chapter describes the experimental setup and procedures used to perform different experiments in this study. One unique aspect of the experimental design was that it captured the key requirements of a SAGD process by involving two horizontal injection and production wells and gravity dominance. Many different types of experiments were performed to collect the data for dead oil density and viscosity, porous matrix permeability and porosity, and oil recovery by varying the type of the solvent and its concentration in a hybrid SAGD process. The details of a novel production system designed to precisely control the pressure of the system carrying steam, gas, oil and water, are included in this chapter. The details for checking the accuracy of the instruments are also part of this chapter.

\subsection{Experimental Setup}

An experimental setup was designed and constructed to perform this study. The key component was a laboratory-scale, cylindrical replica (i.e., physical model) of an oil reservoir with a set of parallel horizontal injection and production wells. The physical model was holding the oil-glass beads mixture and was equipped with two end caps with multiple insertion points for the placement of wells and thermocouples. To simulate a SAGD process involving two horizontal injection and 
production wells, the lower section of the end caps featured two $1.27 \mathrm{~cm}$ holes placed $3.18 \mathrm{~cm}$ apart. The horizontal wells of required length were inserted into the vessel through these holes.

To monitor and record the temperature inside the vessel during the experiments, there were two more ports in the end caps to allow multipoint thermocouples to be inserted inside the model. These two ports were about $3.0 \mathrm{~cm}$ apart, with the bottom port being $2.5 \mathrm{~cm}$ above the injection well port. The five-point thermocouples were inserted from both of these ports.

Figure 3.1 shows the schematic of the physical model. Table 3.1 presents information related to model dimensions (corresponding to the letters in the schematic), distance between the wells and thermocouples, and length of the wells and thermocouples.

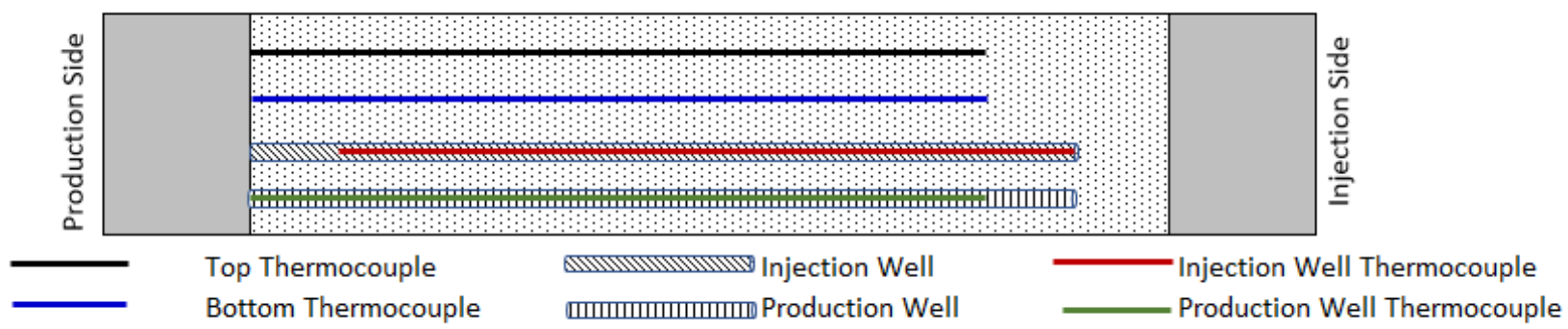

Figure 3.1 Schematic of cylindrical physical model

Table 3.1 Cylindrical model specifications

Model Material Stainless Steel

Model Length $51.00 \mathrm{~cm}$

Model Diameter $11.11 \mathrm{~cm}$

Model Volume $4927 \mathrm{~cm}^{3}$

Top Thermocouple Length $43.81 \mathrm{~cm}$

Bottom Thermocouple Length $43.81 \mathrm{~cm}$

Injection Well Length $50.80 \mathrm{~cm}$

Injection Well Heater Length $45.09 \mathrm{~cm}$

Production Well Length $52.71 \mathrm{~cm}$

Production Well Thermocouple Length $38.74 \mathrm{~cm}$ 
The diameter of the model was selected such that it can accommodate two horizontal wells with enough inter-well distance to minimize early steam breakthrough, while maintaining the same ratio of total reservoir thickness to inter-well distance as for a 15-meter-thick reservoir.

The other important parts of the experimental design and their specifications are listed below:

$\checkmark$ Stainless steel physical model rated for $250^{\circ} \mathrm{C}$ and $7.0 \mathrm{MPa}$.

$\checkmark$ Stainless steel end caps designed to allow insertion of two horizontal injection and production wells and a grid of thermocouples.

$\checkmark$ Perforated $1.27 \mathrm{~cm}$ diameter injection and production wells.

$\checkmark$ A 300-watt heater with a single-point internal thermocouple in the injection well.

$\checkmark$ Two five-point thermocouples in the model.

$\checkmark$ One five-point thermocouple inside the production well.

$\checkmark$ Band heaters around the cylindrical vessel to control heat losses. Four circular heaters were placed on the outer shell of the model, with thermocouples placed between the heater and model shell.

$\checkmark$ Cable heaters surrounding the injection lines to heat the injected fluid to required temperatures.

$\checkmark$ High pressure (34 MPa) injection pump (Isco) to inject cold water equivalent steam.

$\checkmark$ Gas supply tank placed on a scale.

$\checkmark$ Mass flow meter to control the injection rate of the injected gas.

$\checkmark$ High temperature fibre-wool insulation and Kevlar coverings for cylindrical vessel.

$\checkmark$ A production system composed of high pressure Isco pump.

$\checkmark$ Wet experiment meter to measure and record the produced gas.

$\checkmark$ Cable heaters to heat the production lines.

$\checkmark$ Power control cabinets.

$\checkmark$ A data acquisition system to monitor and record the important process parameters.

An electrical heater was inserted into the injection well. This was necessary to ensure that $100 \%$ quality steam was being injected into the model during the experiments. Figure 3.2 shows the overall experimental setup schematic. 


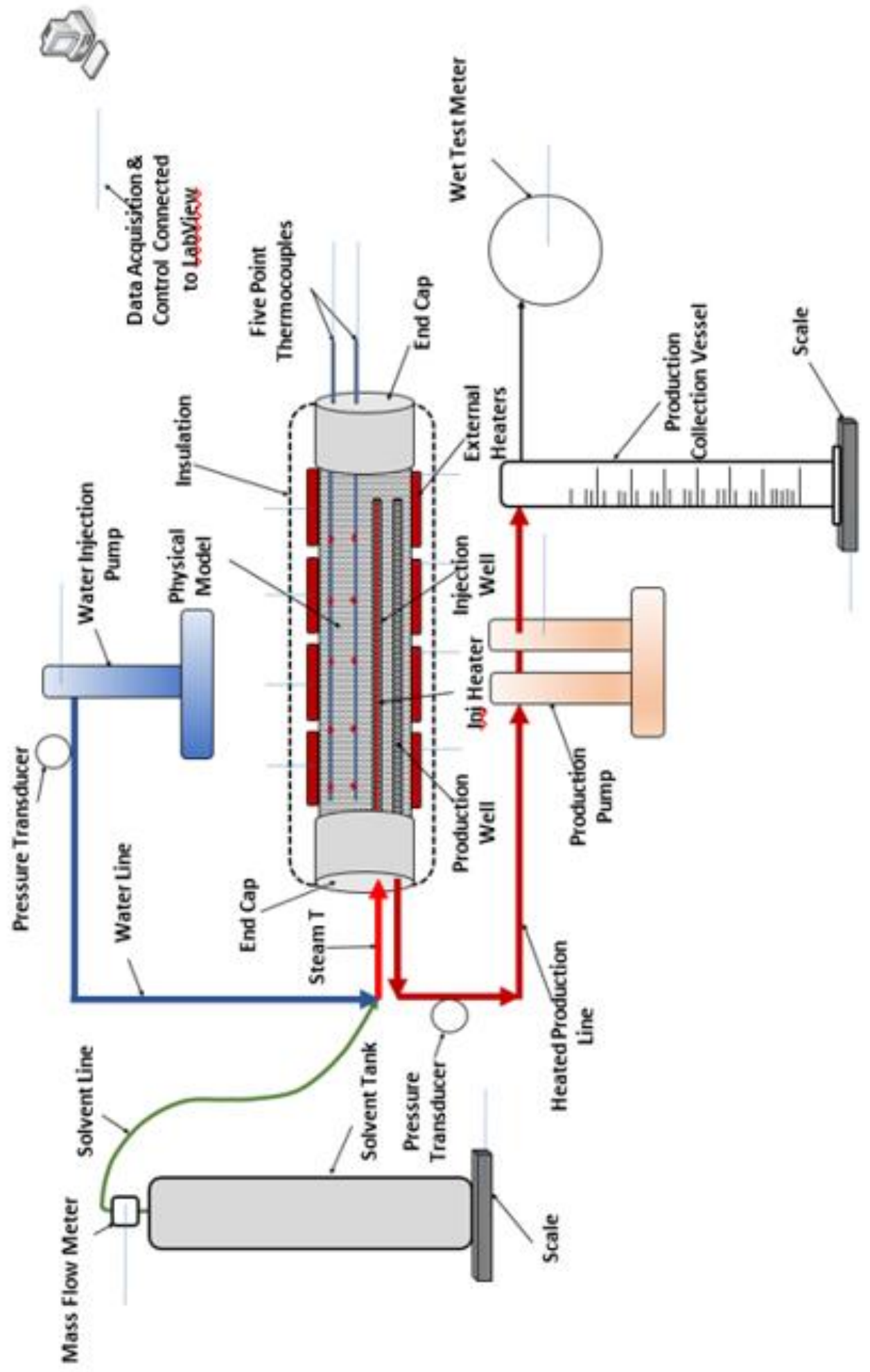

Figure 3.2 Experimental setup schematic 


\subsection{Preparation of Physical Model}

All the experiments in this study were performed by packing the model with an unconsolidated matrix saturated with oil and water. Generally, researchers tend to pack a model with dry unconsolidated sand, inject water to measure the matrix permeability and porosity, and then displace this fluid with oil, and thus by this displacement process obtain the initial model oil and water saturations. For this study, an inventive approach of packing the model was adopted. A sand with known weight was pre-mixed with known amounts of oil and water that would provide initial saturations of about 90 and $10 \mathrm{wt} \%$, respectively. A heavy-duty mixer, Figure 3.3, was used to mix the oil, water and sand. Since the oil used for this study was of high viscosity, the sand and oil were heated to $60^{\circ} \mathrm{C}$ prior to being placed in the mixer bowl. The pre-mixed sand was tightly packed in the model.

The mixing procedure to saturate the sand with oil and water was selected because the model did not have a confining vessel. Thus, with the displacement style oil re-saturation procedure there was a risk that required oil saturation (of about 90\%) wouldn't be achieved.

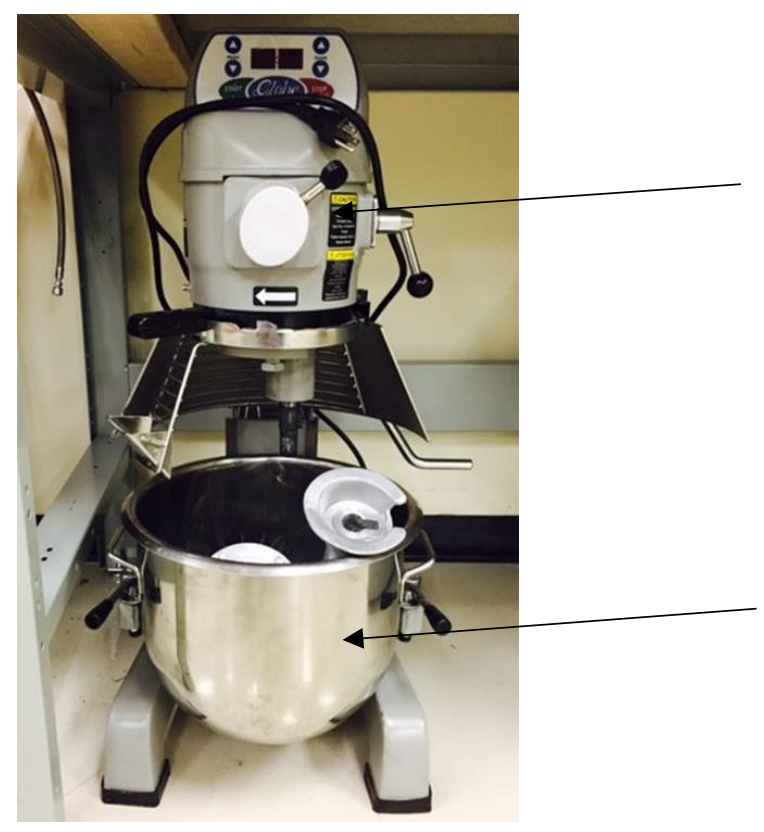

Motor with variable speeds

Mixing bowl

Figure 3.3 Mixing apparatus to prepare sand matrix 
Many experiments were performed during this study, including successful, unsuccessful and experiments repeated to validate the experimental results' reproducibility. A total of 13 experiments with better repeatability were selected to perform the final analysis. The model matrix preparation and model packing were kept consistent among all the experiments. Table 3.2 shows the range of model packing saturations and volumes/weights for all the experiments.

Table 3.2 Model packing saturations and volumes Matrix Weight Including Sand, Oil and Water 9.929 to $10.185 \mathrm{~kg}$

Oil in Model 1.512 to $1.551 \mathrm{~kg}$

Water in Model $\quad 0.166$ to $0.170 \mathrm{~kg}$

Sand in Model 8.250 to $8.462 \mathrm{~kg}$

Model Porosity 31.04 to $31.84 \%$

Oil and Water Saturation $\sim 90.1 \% \mathrm{PV}$, and $\sim 9.91 \% \mathrm{PV}$

\subsection{Synthetic Porous Matrix and Fluid Properties}

Permeability of an oil formation is a measure of the formation's ability to conduct fluids (oil, water and gas) through its pores. Porosity is a characteristic of an oil formation measuring the amount of open pore space that is available to hold oil and water. The porosity is defined as the ratio of void space to bulk space. Note: Synthetic sand manufactured by Opta Minerals Inc., Ontario, with an average absolute permeability of about 10.7 Darcy and a porosity of about $32 \%$ was used for the experiments reported in this work.

A simple 1D core displacement experiment was performed to measure the absolute permeability and porosity of the sand matrix. Major system components included the overburden vessel, Isco pumps for injecting the fluids, a backpressure regulator, collection tubes, a differential pressure transducer across the core, and multiple pressure transducers and thermocouples. The experimental schematic is presented in Figure 3.4.

The synthetic sand was sieved to obtain the size distribution (Table 3.3). A lead sleeve with a diameter of 1 inch and length of 12 inches was prepared by attaching a mandrel on one end. This 
mandrel had a $1 / 4$ inch hole in the middle for injection/production. The sleeve was placed in a vibrator and then sand was added slowly with continuous vibration to fill a quarter of the sleeve's length. The vibration was continued for 30 minutes and then another batch of sand was added to fill half of the sleeve. These steps were repeated until the sleeve was full. A second mandrel was attached; it also had a $1 / 4$ inch hole in the middle for injection/production. To avoid the sand flowing through the mandrel holes, they were covered with a 200-mesh-size screen. The effective length of the sleeve was measured by subtracting the total length of the sleeve plus mandrels and subtracting the length of the mandrels.

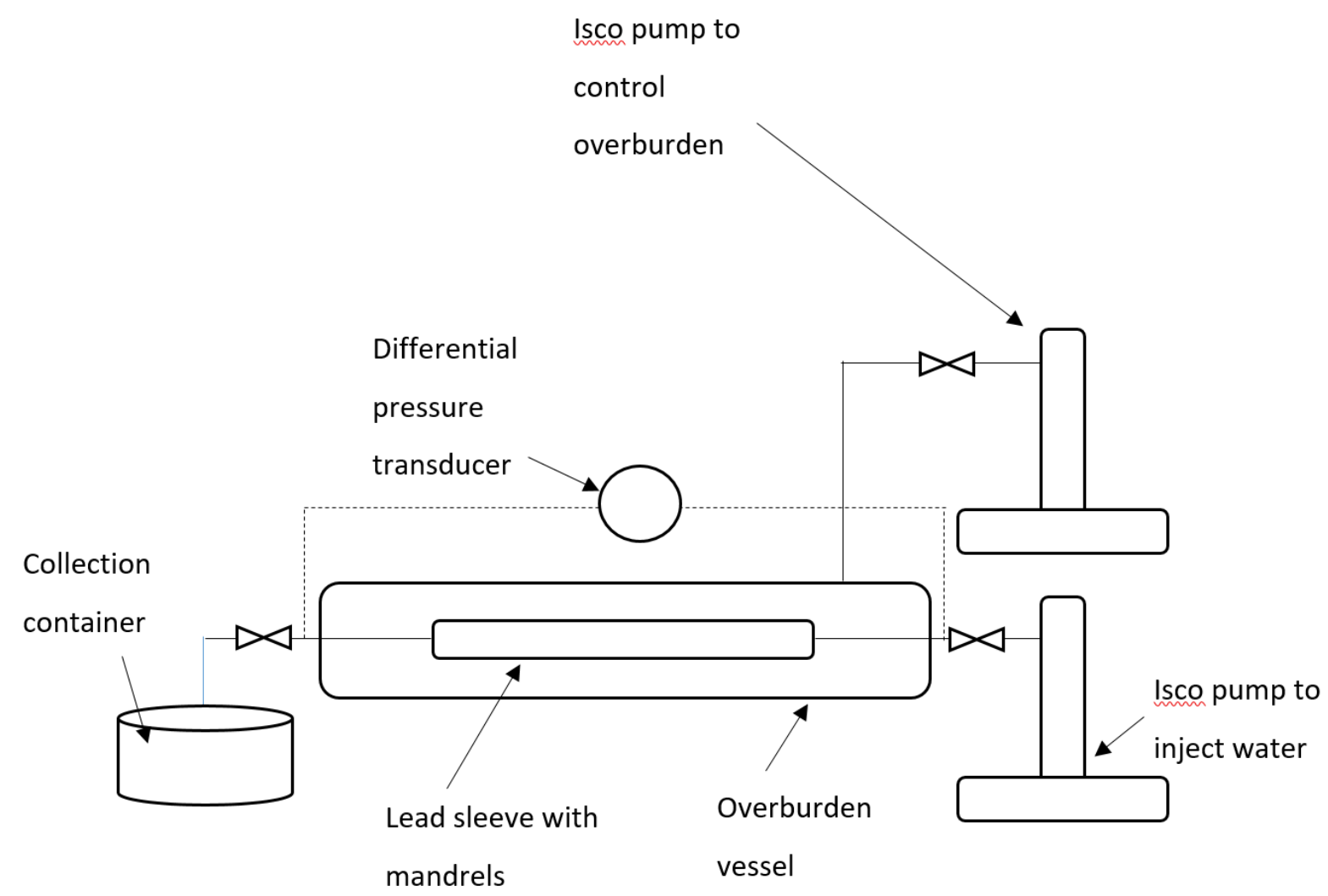

Figure 3.4 Schematic of experimental apparatus to measure permeability 
Table 3.3 Synthetic sand size distribution

\begin{tabular}{c|c|c} 
Microns & Mesh & Weight \% \\
\hline 350 & 45 & 3.01 \\
\hline 250 & 60 & 31.8 \\
\hline 177 & 80 & 37.7 \\
\hline 149 & 100 & 13.7 \\
\hline 125 & 120 & 7.32 \\
\hline 105 & 140 & 3.09 \\
\hline 90 & 170 & 2.13 \\
\hline 74 & 200 & 0.27 \\
\hline
\end{tabular}

The net weight of the sand used to pack the sleeve was recorded. The sleeve was then placed inside an overburden vessel, and all the injection and production lines with associated valves were plumbed and the pressure transducers and thermocouples were installed. The production valve was closed and a hard vacuum ( $-90 \mathrm{kPa}$ ) was pulled from the sleeve through a vacuum pump connected on the injection side. After pulling vacuum for one hour, the vacuum pump was stopped, the injection valve was closed, and the sleeve was left overnight to record any signs of leakage. After it was ensured there were no leaks, and with the sleeve still under vacuum, the injection side was connected to a container of deionized water (DI) placed on a load cell. The injection valve was opened and DI water was allowed to flow inside the sleeve. After the pressure inside the sleeve equilibrated with the atmospheric pressure, the weight of the water container was recorded and the volume inside the sleeve was estimated. This was the pore volume of the sleeve, and with this information, the porosity of the sleeve was calculated.

After the porosity was measured, the overburden was filled with water and was pressurized to 3.0 MPa using an Isco pump. The backpressure regulator was set at $500 \mathrm{kPa}$ and the injection side of the sleeve was connected to an Isco pump to inject water. After the production valve was opened, water was injected at a given flow rate. The injection was continued until a stable differential pressure reading across the sleeve was obtained. Using Darcy's equation below, the permeability of the sand matrix was calculated. 
$k=\frac{q \mu \mathrm{L}}{A \mathrm{~d} P}$

Where $k$ is absolute permeability (Darcy); $q$ is volumetric flow rate $\left(\mathrm{m}^{3} / \mathrm{s}\right) ; A$ is cross sectional area $\left(\mathrm{m}^{2}\right)$; L is length $(\mathrm{m}) ; P$ is pressure $(\mathrm{kPa})$ and $\mu$ is dynamic viscosity $(\mathrm{cP})$.

As a quality control, the permeabilities were obtained against different flow rates, as shown in Table 3.4.

Table 3.4 Synthetic sand permeability at different flow rates

\begin{tabular}{c|c|c|c}
$\boldsymbol{Q}\left(\mathbf{c m}^{\mathbf{3}} / \mathbf{m i n}\right)$ & $\boldsymbol{\Delta P}, \mathbf{k P a}$ & $\mathbf{K}, \mathbf{D a r c y}$ & Std. dev. \\
\hline 3.33 & 0.84 & 10.1 & 0.41 \\
\hline 5.00 & 1.16 & 11.0 & 0.23 \\
\hline 1.67 & 0.41 & 10.3 & 0.27 \\
\hline 6.67 & 1.50 & 11.3 & 0.44 \\
\hline \multicolumn{2}{r|}{ Average } & 10.7 &
\end{tabular}

The oil used for this study was from Waseca formation in the Lloydminster Saskatchewan area. The oil viscosity was measured at atmospheric pressure and $15-40^{\circ} \mathrm{C}$ and at $500 \mathrm{kPa}$ and $40-$ $200^{\circ} \mathrm{C}$. A Brookfield viscometer, manufactured by Brookfield Ametek, USA, was used for these measurements. The bath was set at the required temperature. A $0.5 \mathrm{~mL}$ sample was placed in the middle of the sampling cup. The viscometer rotor was set at the required speed. For a given sample, the display value of viscosity, revolutions per minute (rpm) and torque\% were recorded. Once an experiment was complete, the cup was cleaned with toluene, which was then rinsed with acetone to ensure no toluene was left on the cup, since it can contaminate the next sample. After the cup was cleaned, a sample with known viscosity was tested by setting up the viscometer at the same $\mathrm{rpm}$, torque and temperature as of the previously tested sample. A correction factor was calculated as:

$\frac{\text { Known standard viscosity }}{\text { Measured standard viscosity }}$ 
This factor was applied to the previously measured sample reading.

The viscosity data as a function of temperature is shown in Figure 3.5. To estimate the oil viscosity at elevated temperatures where it could not be measured, a power law correlation was established by curve fitting the experimental data. The correlation is presented below:

$\mu=2.8 \times 10^{11} \times T^{-4.686}, \mathrm{R}^{2}=99 \%$

Note that in the correlation, $\mu$ stands for viscosity $(\mathrm{mPa} \cdot \mathrm{s})$, and $\mathrm{T}$ for temperature $\left({ }^{\circ} \mathrm{C}\right)$.

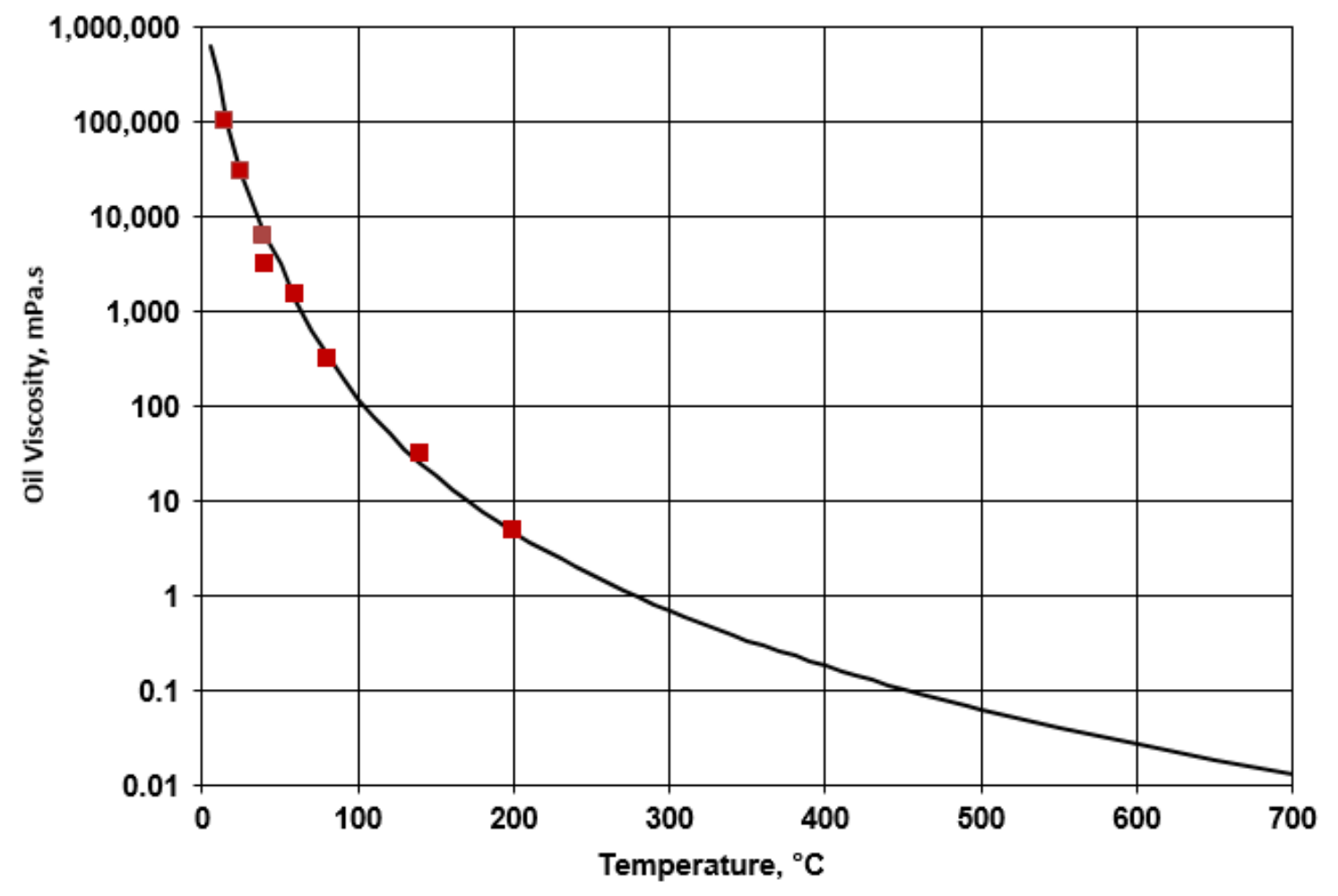

Figure 3.5 Oil viscosity versus temperature

The oil density was measured using an Anton Parr digital density analyzer (Anton Paar Canada Inc, Quebec), with accuracy to five decimal places. The ASTM D5002 experimental method was implemented. 
Before the experiment, the instrument was checked for calibration. The experiment tube was examined for any condensation. A sample of distilled water (1 to $2 \mathrm{~mL}$ ) was introduced to the sample tube. After it was ensured there were no air bubbles, the instrument was run to measure and validate the water density. Once the calibration check was complete, the required amount of oil sample was poured into the sample tube. After the instrument was set at the required temperature, it was run to measure the sample density.

Density of the Waseca oil was measured at atmospheric pressure and $15-40^{\circ} \mathrm{C}$ (Table 3.5 ) and at elevated pressures and temperatures: up to $6 \mathrm{MPa}$ and $200^{\circ} \mathrm{C}$ (Figure 3.6). The functional relationship between the density and temperature is shown below. Note that in the correlation below, the units of oil density and temperature are $\mathrm{kg} / \mathrm{m}^{3}$ and ${ }^{\circ} \mathrm{C}$, respectively.

Oil density $=-0.6 T+1007$

Table 3.5 Oil density at different temperatures and atmospheric pressure

\begin{tabular}{rl}
$\boldsymbol{T}\left({ }^{\circ} \boldsymbol{C}\right)$ & Density $\left(\mathbf{k g} / \mathbf{m}^{\mathbf{3}}\right)$ \\
\hline $\mathbf{1 5}$ & 998 \\
\hline $\mathbf{2 5}$ & 992 \\
\hline $\mathbf{4 0}$ & 983
\end{tabular}

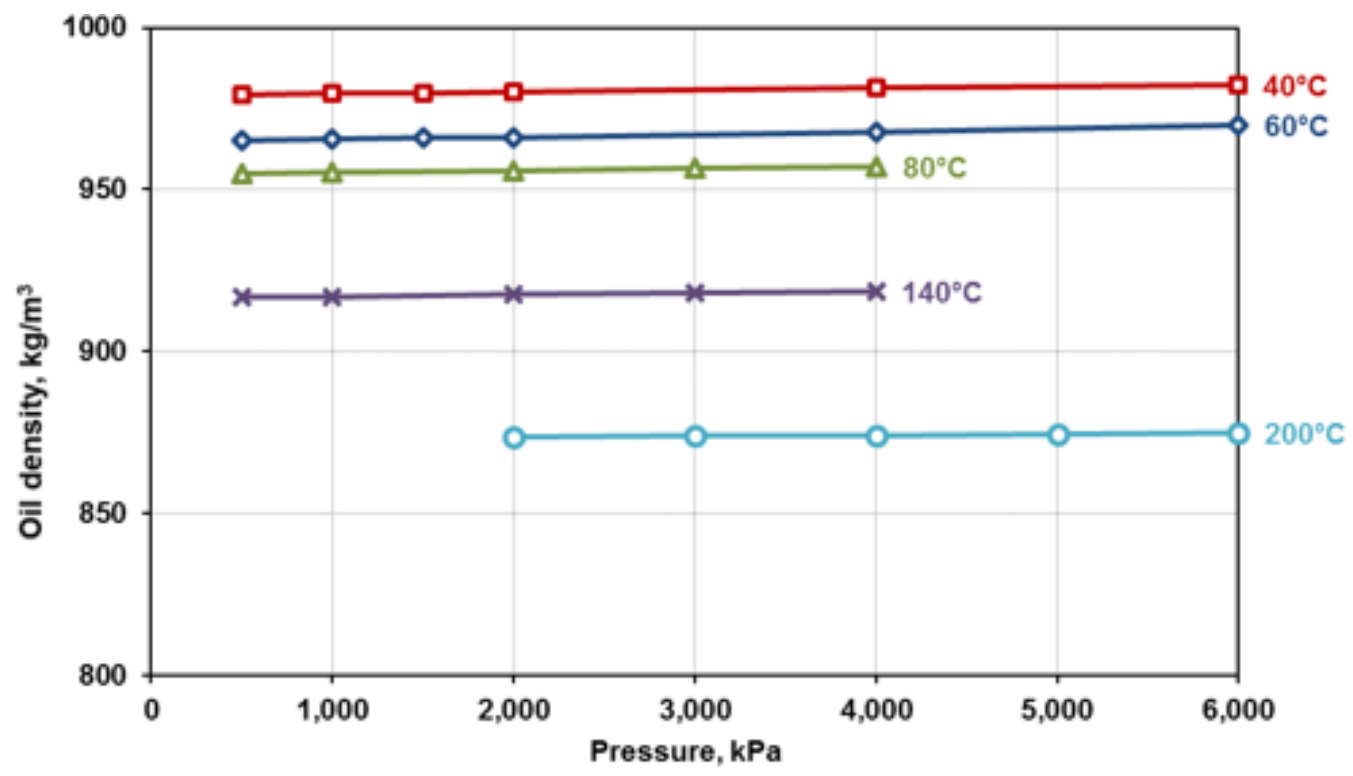

Figure 3.6 Oil density at elevated pressures and temperatures 


\subsection{Experimental Procedure}

The experimental procedure for all of the experiments was the same except for the change in the injection rate and solvent. The steps of the procedure are as follows:

\section{Step 1}

At first the matrix was prepared by mixing known amounts of synthetic sand, oil and water, as described in the previous section. With the cylindrical model installed on the stand, one of the end caps was connected, and the thermocouples and injection and production wells were installed (as shown in the schematic, Figure 3.2). With the model in the vertical position, it was packed with the pre-mixed unconsolidated sand. A small quantity, 200 to $300 \mathrm{~g}$, was poured inside the model and pressed with a flat spatula to ensure that the trapped air within the pores was evacuated.

\section{Step 2}

Once the model was completely packed, the other end cap was installed with the connection of injection and production lines. The side heaters that covered the model outer body were also installed with a thermocouple placed between the heater and model shell. This thermocouple was used as a control point to regulate the model surface temperature for conducting isothermal experiments.

\section{Step 3}

An inline heater was installed inside the injection well. This heater's temperature was controlled by a built-in thermocouple. The purpose of this heater was to ensure that the steam injected into the model was always 10 to $20^{\circ} \mathrm{C}$ above the saturated steam temperature at the injection conditions. The injection and production lines were covered with cable line heaters with thermocouples installed between the heaters and tubes for controlling the heater temperatures. The injection line was connected to an Isco pump that was used to inject cold water equivalent to steam. Through a cross, the same line was connected to a gas supply tank that was placed on a balance to record the weight of the gas being injected as a function of time. The pressure in the gas tank was controlled by a regulator. The flow of the gas injected was controlled by a gas flow meter. 
The production line was connected to an Isco pump as well. This double cylinder Isco pump, manufactured by Telydyne Isco, USA, was used to collect the produced fluids and dump them into a separator.

One of the challenges in performing the thermal experiments is the control of the production pressure. If the pressure is not precisely controlled, this can cause intensive fluctuations (up to +/$200 \mathrm{kPa}$ ) and result in an unstable steam chamber. Generally, the system pressure is controlled either using a flow control valve or a backpressure regulator. The problem with these two devices is that they cannot handle multi-phase flow systems very well and this causes major pressure instability. To overcome this problem, the system pressure on the production side of the model was controlled using a double cylinder Isco pump. The controller of the pump was programmed to collect the fluids from the system at a defined pressure and with a maximum flow rate. This program allowed the valves to be switched between the pump barrels once the barrel assigned for fluid collection is full. It then discharged the fluids to a collection vessel while the other barrel starts to collect the fluid. This production system controlled the system pressure precisely. An example of the standard deviation of the production pressure from the setpoint is presented in Figure 3.7 below.

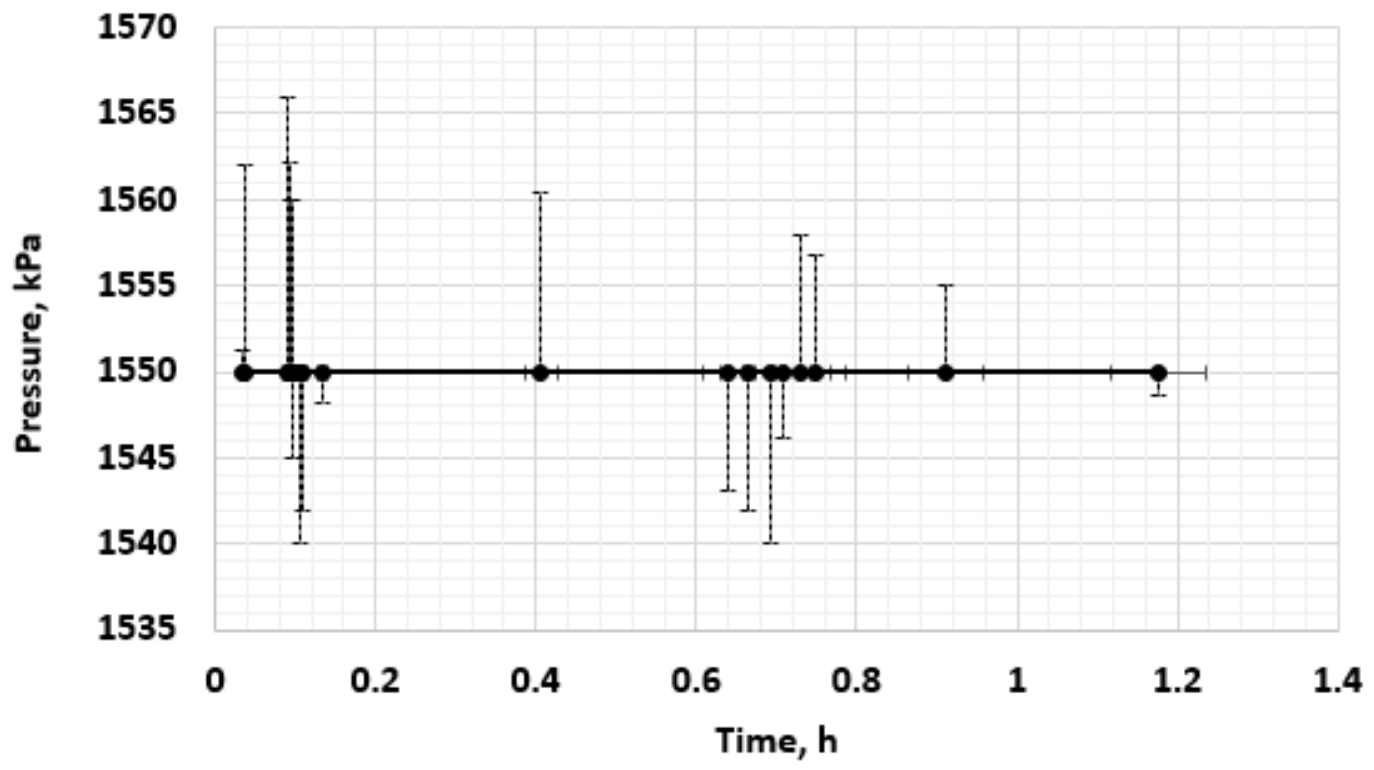

Figure 3.7 Production pressure standard deviation chart 


\section{Step 4}

For each experiment, first a preheating stage was performed. The temperature inside the injection well was increased from room temperature to $95^{\circ} \mathrm{C}$, in $25^{\circ} \mathrm{C}$ increments every five minutes. Then the model was left at this temperature setting for 24 hours. The model surface heaters were not turned on during the pre-heating stage. The heaters on the production line and the production pump were turned on and set at $80^{\circ} \mathrm{C}$. The pressure on the production pump was set at about $1.45 \mathrm{MPa}$, gauge, which is the saturated steam pressure at about $195^{\circ} \mathrm{C}$.

Once the model was preheated and it was ensured that the temperature between the wells was close to or above the mobilization temperature of oil, about $80^{\circ} \mathrm{C}$, it was prepared for steam and or gas injection. About one hour before the injection valve was opened, the temperature of the heater inside the well and the temperature around the model shell were increased. The temperature ramp up for all the experiments was kept consistent. Table 3.6 shows how these temperatures were increased. The purpose of this temperature increase was to set isothermal conditions for steam only or hybrid SAGD experiments.

Table 3.6 Preheating schedule of the model

Time Inj Heater $\quad$ Shell Heater 1 Shell Heater 2 Shell Heater 3 Shell Heater 4

\begin{tabular}{cccccc}
$\min$ & ${ }^{\circ} \mathrm{C}$ & ${ }^{\circ} \mathrm{C}$ & ${ }^{\circ} \mathrm{C}$ & ${ }^{\circ} \mathrm{C}$ & ${ }^{\circ} \mathrm{C}$ \\
\hline 0 & 95 & 95 & 95 & 95 & 95 \\
\hline 5 & 100 & 100 & 100 & 100 & 100 \\
\hline 10 & 105 & 105 & 105 & 105 & 105 \\
\hline 15 & 115 & 115 & 115 & 115 & 115 \\
\hline 20 & 125 & 125 & 125 & 125 & 125 \\
\hline 25 & 135 & 135 & 135 & 135 & 135 \\
\hline 30 & 145 & 145 & 145 & 145 & 145 \\
\hline 35 & 155 & 155 & 155 & 155 & 155 \\
\hline 40 & 165 & 165 & 165 & 165 & 165 \\
\hline 45 & 175 & 175 & 175 & 175 & 175 \\
\hline 50 & 185 & 185 & 185 & 185 & 185 \\
\hline 55 & 195 & 195 & 195 & 195 & 195
\end{tabular}




\section{Experimental Conditions}

All the experiments were performed in an isothermal environment. The band heaters on the external surface of the cylindrical model were set such that the temperature inside the model did not exceed $195^{\circ} \mathrm{C}$. The primary reason to set this temperature limit was to address low-pressure reservoirs where the overburden is not thick enough to support saturated steam pressures above $200^{\circ} \mathrm{C}$. Currently there are huge heavy oil resources, over approximately 600 billion barrels, that are too shallow to mine and too deep to apply conventional SAGD. The secondary reason to select a temperature below $200^{\circ} \mathrm{C}$ for the experiments was to avoid aqua-thermolytic reactions and eliminate the chances of hydrogen sulfide production. In a conventional SAGD process that is applied at temperatures of $240^{\circ} \mathrm{C}$ and above, the generation of hydrogen sulfide adds another level of complexity, safety issues and additional costs. Eliminating or reducing the chances of hydrogen sulfide generation would have a positive bearing on this EOR process. Table 3.7 shows the injection rates for all of the experiments.

Table 3.7 Gaseous solvent injection rates with CWE steam rate of $115 \mathrm{~mL} / \mathrm{min}$

\begin{aligned} Experiments & Solvent Rate (std L/h) \\ \hline Baseline (Steam Only) & 0.00 \\ \hline Steam plus 5.0 wt\% carbon dioxide & 3.12 \\ \hline Steam plus 7.5 wt\% carbon dioxide & 4.68 \\ \hline Steam plus 10.0 wt\% carbon dioxide & 6.24 \\ \hline Steam plus 5.0 wt\% nitrogen & 4.92 \\ \hline Steam plus 7.5 wt\% nitrogen & 7.32 \\ \hline Steam plus 10.0 wt\% nitrogen & 9.78 \\ \hline Steam plus 5.0 wt\% methane & 8.52 \\ \hline Steam plus 7.5 wt\% methane & 12.8 \\ \hline Steam plus 10.0 wt\% methane & 17.1 \\ \hline Steam plus 5.0 wt\% propane & 11.2 \\ \hline Steam plus 7.5 wt\% propane & 16.7 \\ \hline Steam plus 10.0 wt\% propane & 22.4\end{aligned}


For all experiments, the injection was continued for about 7.0 to 8.0 hours. Upon injection, the model pressure started to increase and once it reached the required setpoint of $1.45 \mathrm{MPa}$ gauge, the pump started to collect the produced fluids. The fluids from the production pump passed through a separator that was connected to a wet test meter.

\section{Baseline Experiment}

First a baseline experiment with steam only was performed. The injection rate of steam was set assuming a steam to oil ratio (SOR) of about $3.0 \mathrm{sm}^{3} / \mathrm{sm}^{3}$ and recovery of about $60 \%$ of in-place oil in about 24 hours. The injection rate of steam was calculated to be $115 \mathrm{~mL} / \mathrm{min}$. For the steam plus solvent injection experiments, the water rate was set equal to that in the baseline experiment and the solvent injection rate was calculated corresponding to the weight percentage of the solvent.

\section{Sample Collection and Analysis}

The produced samples containing a mixture of oil and water were processed in a high-speed centrifuge, by L-K Industries, Texas, USA, to measure the fractional volumes of oil and water. A separate 100-mL centrifuge tube was used for each sample. Oil and water from the collection vessel were transferred to the $50 \mathrm{~mL}$ mark of the tube. Four centrifuge tubes were filled in this fashion and placed inside the high-speed centrifuge. These samples were heated to $50^{\circ} \mathrm{C}$ for 15 to 20 minutes by turning on the centrifuge heaters. Afterwards, $50 \mathrm{~mL}$ of varsol was added to each tube. Thus, a total of $100 \mathrm{~mL}$ of fluid was in each tube. These tubes were weighed and it was ensured that all weights were within 0.5 grams of each other. These tubes were then spun at 1500 rpm for 30 minutes. Then, the water level for each tube was recorded and corrected for $50 \mathrm{~mL}$ of total oil being used. By knowing the amount of the water and the weight of the original sample, the amount of the oil was back-calculated.

\subsection{Data Accuracy and Quality Check}

The accuracy and quality of the data generated were ensured by checking the accuracy of the important instruments, for example, the load cells, thermocouples and the pressure transducers, a 
couple of times during the experiment. The accuracy of the load cell to three decimal places was verified using a set of working weights, which were ASTM Class 1 weights.

The accuracy of selected thermocouples was verified against a certified thermometer calibrated by a recognized standard agency such as NIST. The certified thermometer and that to be tested were placed in a water container. After the temperature was equilibrated, the reading of each thermometer was recorded. Readings were obtained for at least two points and were found to be within $1^{\circ} \mathrm{C}$ of the certified thermometer.

The pressure transducers or differential pressure cells were verified against a calibrated reference gauge. The process transducers readings were found to be $0.1 \%$ of the reference gauge and of each other. Table 3.8 presents the results for the pressure transducer and differential pressure accuracy check.

One of the important aspects of this study was the design of the control systems to regulate the model and the well temperatures. The temperature of the model, injection well and production lines was controlled by heaters. The heaters were controlled using PID (proportional-integralderivative) controllers. To precisely control the heater temperatures, the PIDs were tuned by adjusting the gain and integral time. Figure 3.8 shows the standard deviation of the process value from the setpoint for the model's external heaters.

Table 3.8 Pressure transducer and differential pressure accuracy

\begin{tabular}{cccc}
$\begin{array}{c}\text { Transducer } \\
\text { Type }\end{array}$ & $\begin{array}{c}\text { Transducer Reading } \\
\text { MPa }\end{array}$ & Reference Gauge MPa & Deviation \\
\hline$P T 1$ & 5.017 & 5.015 & $0.03 \%$ \\
\hline$P T 2$ & 5.019 & 5.015 & $0.08 \%$ \\
\hline$\Delta P$ & 1.523 & 1.522 & $0.07 \%$
\end{tabular}




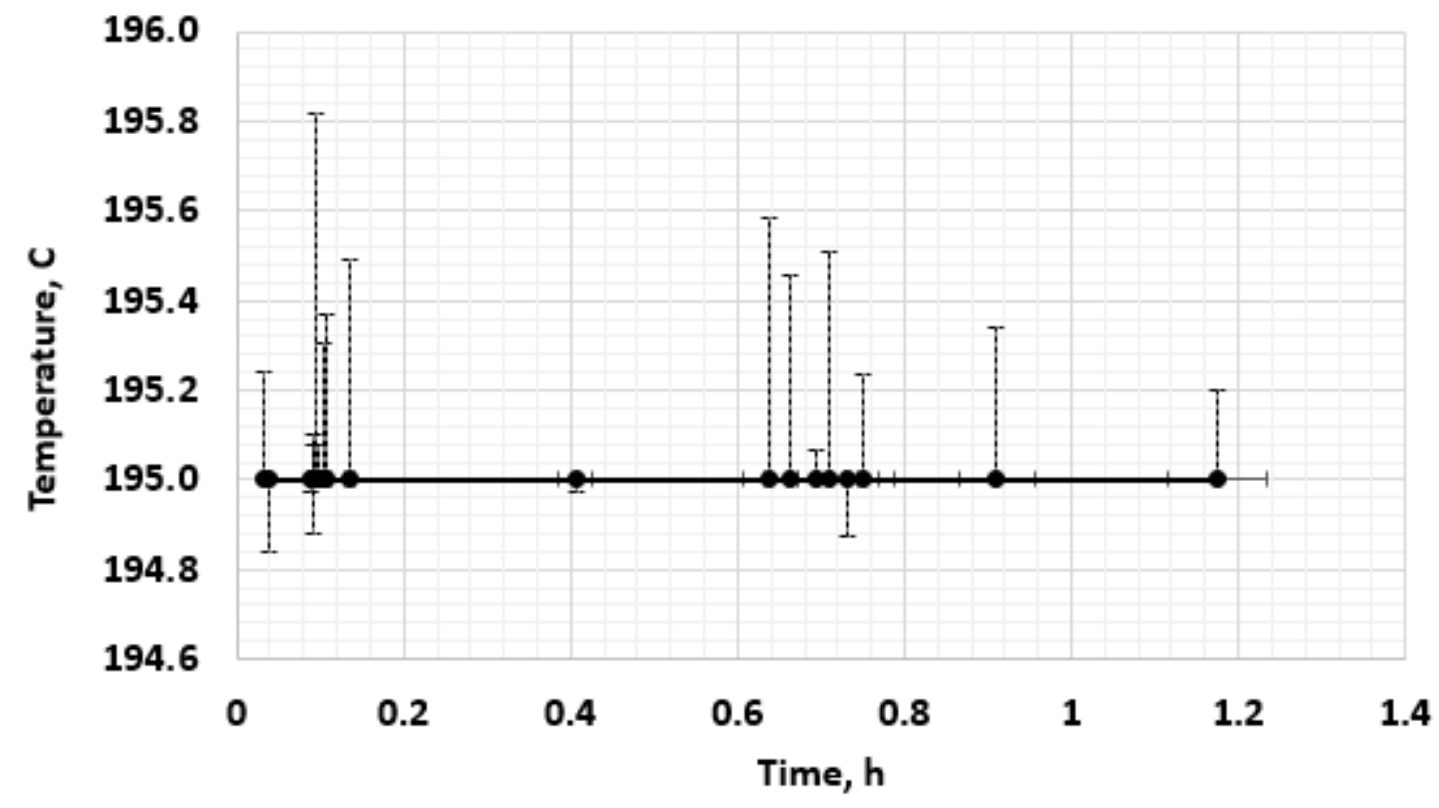

Figure 3.8 External heater standard deviation 


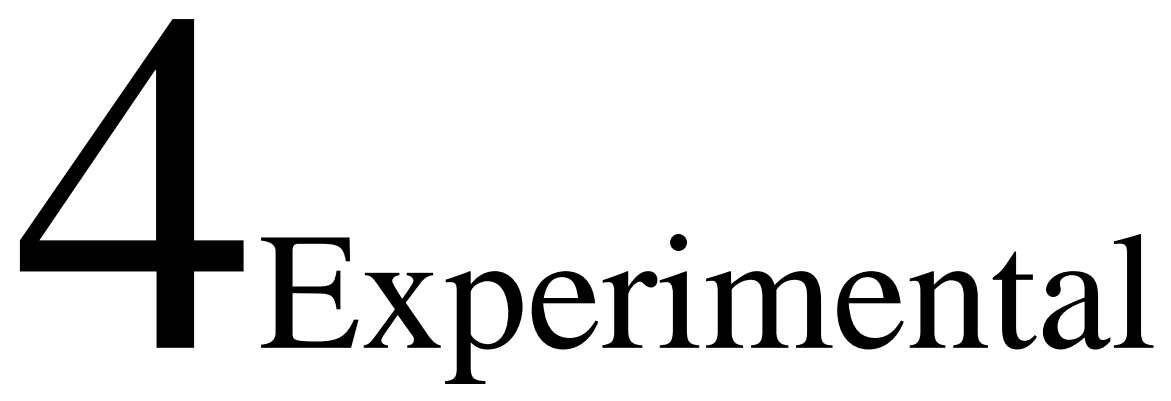

\section{Results \&}

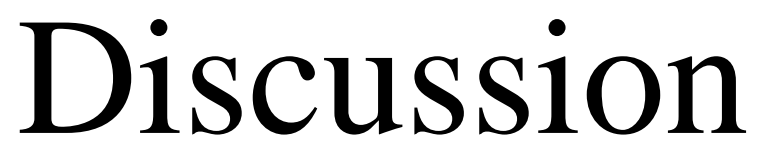

This chapter presents the results and analysis of the experiments performed in this work. The petrophysical properties and operational parameters were kept consistent among all the experiments, other than the intentional variations in the solvent type and their concentrations.

Sections 4.1 to 4.5 present the important results for the addition of different solvents with steam at varying concentrations and compare their performance with the baseline experiment with steam only. The objective of this comparison was to evaluate the effect of change in concentration on hybrid SAGD performance for a given solvent. Section 4.6 compares the results for all the solvents at a given concentration. The objective of this comparison was to evaluate the effect of solvent type on hybrid SAGD performance. 


\subsection{Baseline Experiment - Steam Only SAGD}

This experiment, in which only steam was injected into the model, was set as a baseline to assess hybrid SAGD experiments using solvents co-injected with steam. After the model was preheated for about 24 hours at close to $95^{\circ} \mathrm{C}$, steam at a cold-water-equivalent (CWE) injection rate of 115 $\mathrm{mL} /$ hour was injected. The initial pressure inside the model prior to pre-heating was atmospheric pressure. As the model got heated, the pressure started to increase. As mentioned earlier, the injection rate was calculated by assuming a produced steam to oil ratio of $3.0 \mathrm{sm}^{3} / \mathrm{sm}^{3}$ and producing about $60 \%$ of the OOIP in approximately 24 hours. Since the scale of the model was small, there was a chance that, at this rate, the steam could quickly break through from the production well, bypassing the matrix above the injection well. Therefore, a trial experiment was performed to ensure that at $115 \mathrm{~mL} / \mathrm{min}$, steam does not break through; it was found satisfactory.

With steam only injection, it took around 4.0 hours before the model could achieve the set pressure of $1.45 \mathrm{MPa}$, gauge, and started to produce. Figure 4.1 presents the injection and production pressures and the differential pressure (or $\Delta \mathrm{P}$ ) across the wells during the injection stage of the baseline experiment. The $\Delta \mathrm{P}$ was about $18 \mathrm{kPa}$ when the oil first started to produce and then it remained below $2 \mathrm{kPa}$ for the rest of the time, indicating that there was no steam breaking through from the injection to the production well.

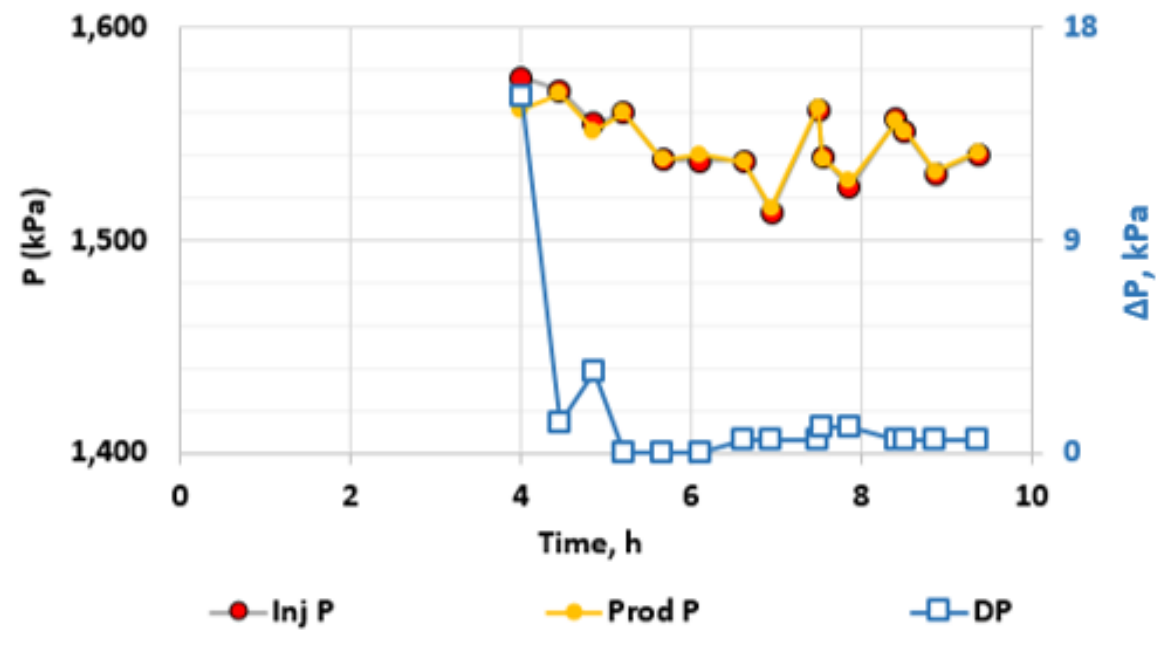

Figure 4.1 Baseline experiment - model pressures and $\Delta \mathrm{P}$ 
Figure 4.2 shows the cumulative produced oil and percent original oil in-place (OOIP) recovery as a function of time for this baseline experiment. The oil recovery started to pick up once the model pressure reached the target value and pushed the fluids out to the production well. Within 9.5 hours of injection, only $10.6 \%$ OOIP was recovered; this was equivalent to $147 \mathrm{~mL}$ of oil. This recovery is much lower than for conventional SAGD type of process, where up to $60 \%$ of recovery can be achieved during the rising and spreading phase of the process. The reason for such a low recovery from the laboratory scale model could be primarily the low temperature and pressure conditions of the experiments.
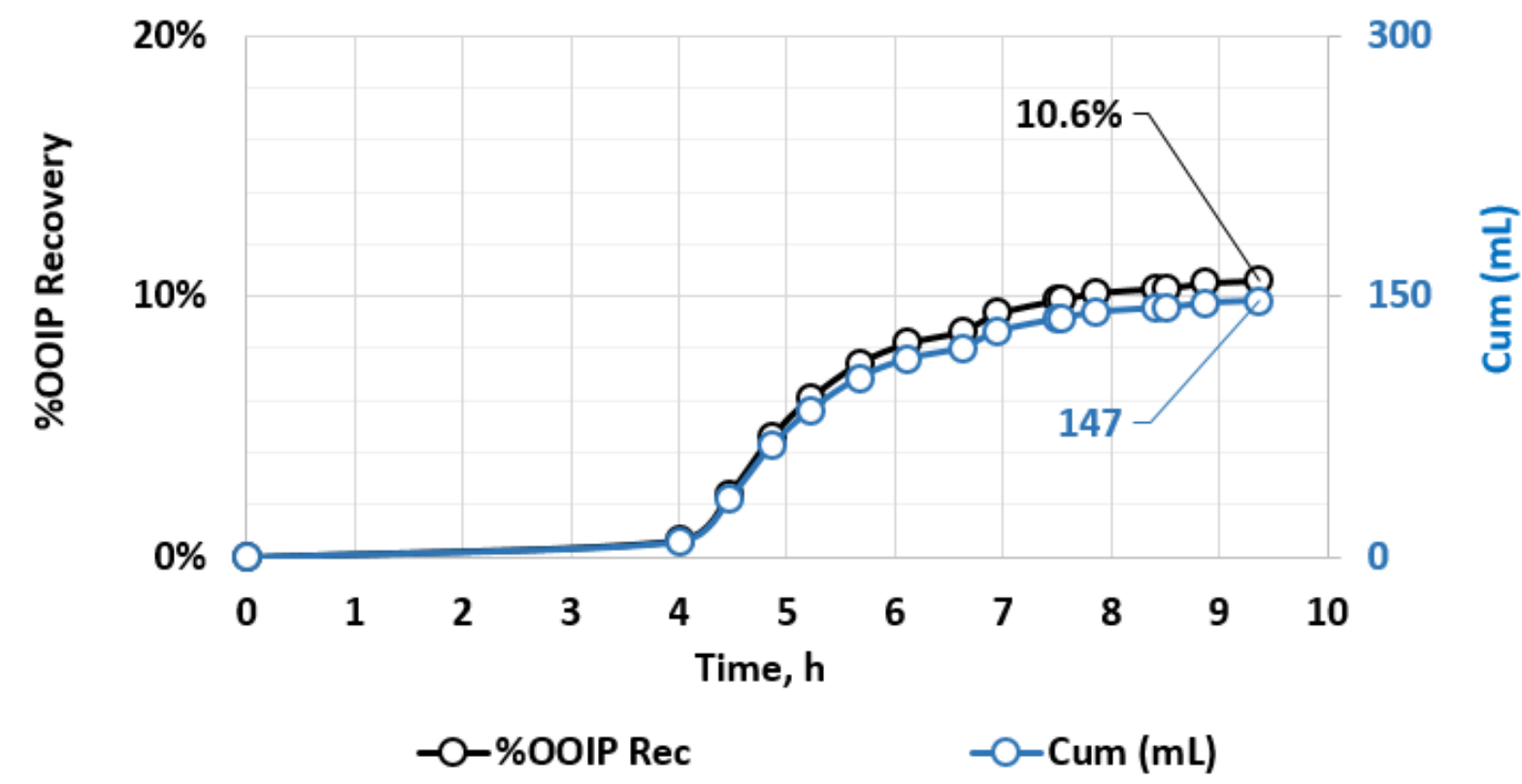

Figure 4.2 Baseline experiment cumulative and \%OOIP recovery

Figure 4.3 shows the cumulative volumes of injected and produced water during this experiment. In total, $1078 \mathrm{~mL}$ of water was injected and only about $50 \%$ of this was produced. The slopes of the injection and production curves were similar once the model reached the target pressure and production started. The reason for such a large difference between injection and production was that around half of the injected water contributed to pressurizing the model to the target pressure of 1.45 MPa, gauge. This indicates that for low-pressure SAGD application, especially where the reservoirs are depleted to very low pressures, a lot of injected energy and fluid will be required to pressurize the reservoir. The energy associated with the injection will probably be lost for a while, especially when the reservoir is being pressurized. 


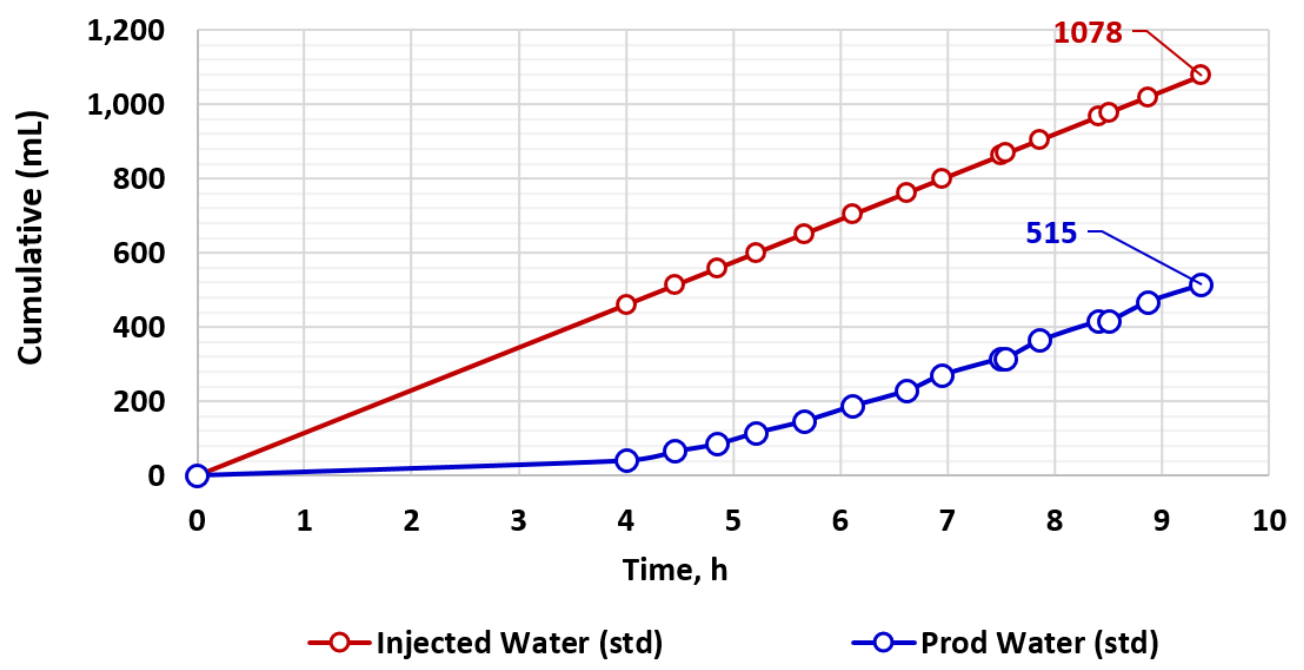

Figure 4.3 Cumulative injected and produced water - Baseline experiment

Figure 4.4 shows the average temperature from the top and bottom row of thermocouples, injection well temperature, and average production well temperature. Refer to Figure 3.1 for the locations of these thermocouples. The injection well always stayed above $215^{\circ} \mathrm{C}$ and the production well was always about $30^{\circ} \mathrm{C}$ less than the injection well temperature. This indicates that there was a stable liquid level maintained between the wells and that live steam never broke through between the injection and production wells. Note that the model temperatures during the preheating and injection stages for all of the experiments are presented in Appendix A of this dissertation.

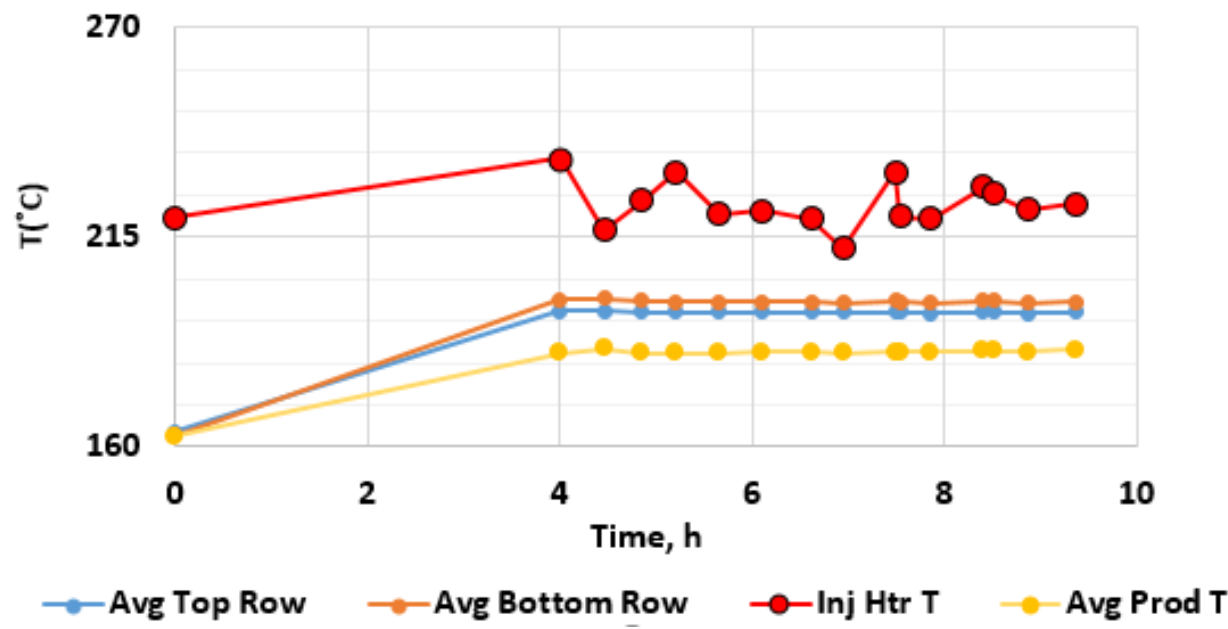

Figure 4.4 Model temperatures - Baseline experiment 


\subsection{Steam Plus Carbon Dioxide Injection Experiments}

This section presents the comparison of produced fluids, i.e. produced oil, water and gas; and pressures during the experiments in which carbon dioxide was injected with steam. The objective here is to investigate the performance of hybrid SAGD by varying the carbon dioxide concentration in the injected stream and also to compare the results with the baseline experiment in which only steam is injected.

Figure 4.5 compares the \%OOIP recovery from the experiments with carbon dioxide addition with the steam only experiment. Note that the model packing, preheating and injection schedule was fairly consistent among all these experiments.

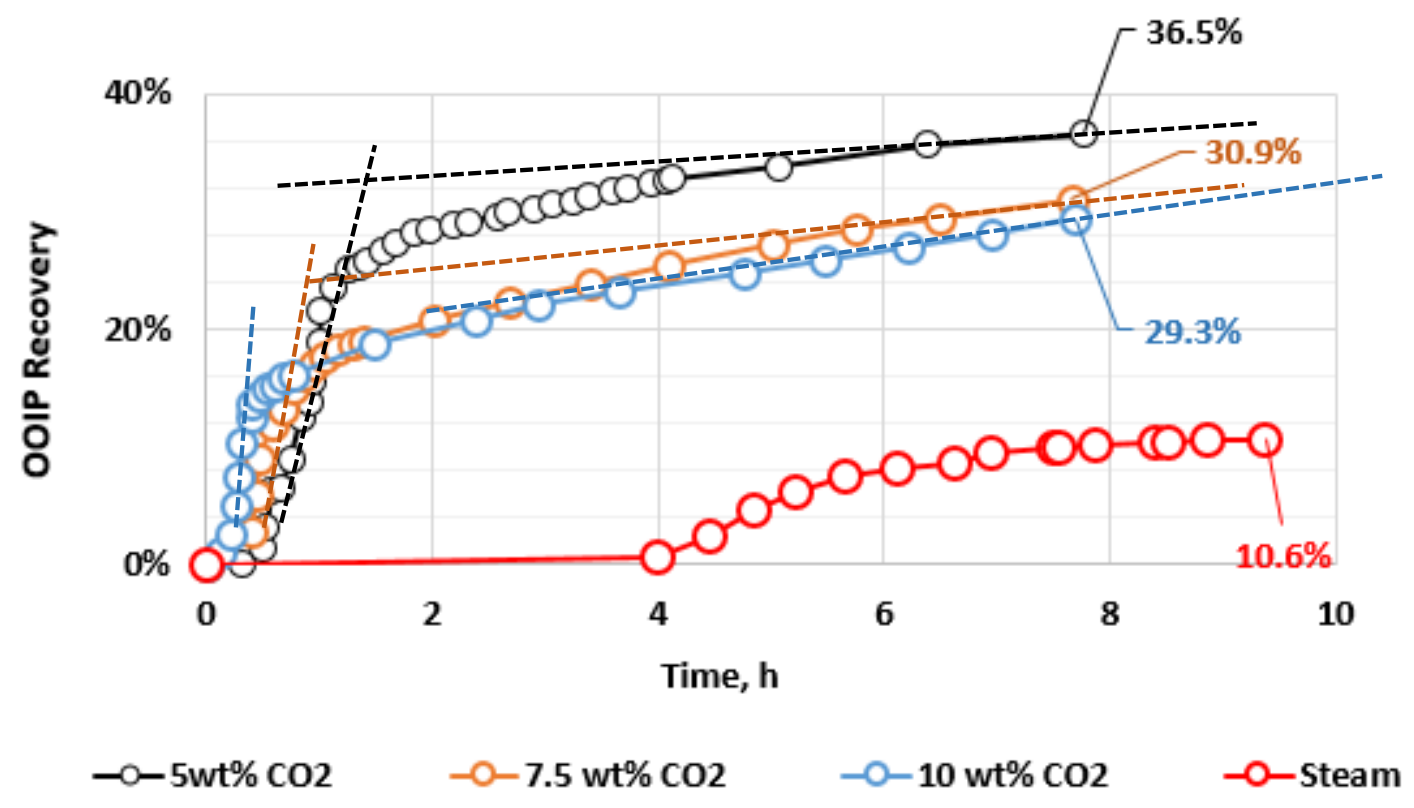

Figure 4.5 Oil recovery comparison of steam only with steam plus $\mathrm{CO}_{2}$ experiments

Among the three concentrations of $\mathrm{CO}_{2}$ used, the lowest of $5.0 \mathrm{wt} \%$ showed the highest oil recovery of $36.5 \%$ OOIP, whereas the highest concentration of $10.0 \mathrm{wt} \%$ showed the lowest recovery of $29.3 \%$ OOIP. The recovery from the intermediate concentration of $7.5 \mathrm{wt} \%$ was $30.9 \%$ OOIP, slightly higher than for the $10.0 \mathrm{wt} \%$ experiment. 
Generally, an increased solvent concentration in the injected stream would have a positive effect on oil recovery. With this point of view, the results from carbon dioxide experiments are seemingly contrary. Carbon dioxide is soluble in oil, although at the experimental conditions its solubility is approximately three times lower than at a lower temperature (Figure 4.6). Nevertheless, even then there will be some additional reduction in oil viscosity, which would be thought to contribute to producing more oil with higher loading of carbon dioxide. Note that the k-value table presented in Figure 4.6 was generated for the fluid model that was used to conduct the numerical simulations and history-match the experimental data. The k-values are extrapolated from experimental data obtained in a study done by Freitag (2008). The k-value is a measure of solubility. By definition, $\mathrm{k}$-value of a solvent is the ratio of solvent mole fraction in the gas phase to solvent mole fraction in the oil phase. From this ratio the solubility of the solvent in the oil phase is calculated. The solubility of the solvent can be expressed as grams of solvent/grams of oil, $\mathrm{mL}$ of solvent/mL of oil or moles of solvent/moles of oil. Knowing the solubility of a given solvent the viscosity of the solvent oil mixture can be calculated.

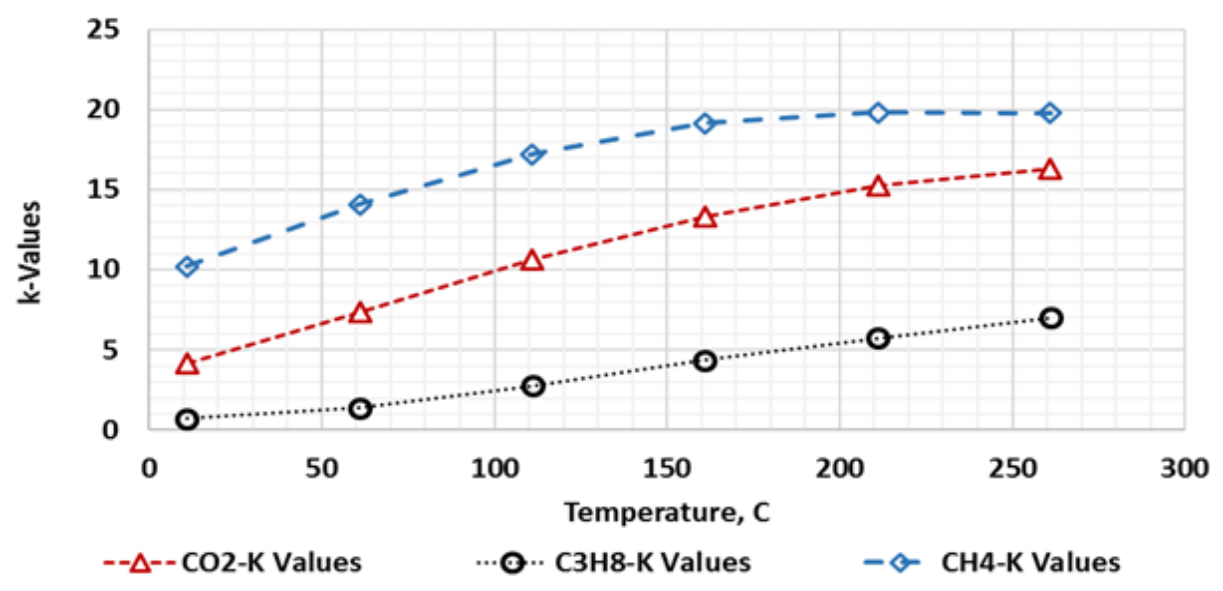

Figure 4.6 K-values of different solvents at $1500 \mathrm{kPa}$

Employing the viscosity correlation proposed by Freitag (2008), the viscosity of dead oil at run conditions is theoretically approximately $10 \mathrm{cP}$, and with the dissolved solvent at run conditions, this viscosity can drop to about $6 \mathrm{cP}$, that is, around a $40 \%$ reduction. However; the experimental results showed an inverse effect of carbon dioxide loading. A plausible reason for this anomaly is the increased relative permeability of gas with higher carbon dioxide loading. The results indicate 
that, above $5.0 \mathrm{wt} \%$ addition of this specific solvent, the relative permeability of gas suppresses the relative permeability of oil and does not allow all the mobile oil to be produced, especially at earlier times of the process. Above $7.5 \mathrm{wt} \%$ addition of carbon dioxide, the oil recovery still decreases slightly. The results of this study suggest that there is a threshold for carbon dioxide addition to steam, after which the oil recovery could be negatively affected.

As shown in Figure 4.5, the initialization of oil production for the 5.0, 7.5 and $10.0 \mathrm{wt} \%$ carbon dioxide experiments occurred at $0.53,0.41$ and 0.12 hours, respectively. However, both the 7.5 and $10.0 \mathrm{wt} \%$ runs reached a plateau earlier than the $5.0 \mathrm{wt} \%$ experiment. This could be attributed to the higher carbon dioxide injection rate. Another observation from the same plot is that the slopes of the curves (indicated by the dotted lines) at later times of the process are also slightly steeper for the higher concentration experiments.

Compared to the baseline experiment, all the experiments with carbon dioxide addition produced substantially large volumes of oil. The recovery from $5.0 \mathrm{wt} \%$ carbon dioxide addition experiment was approximately 26 percentage points more than from the baseline experiment. Similarly, from the $7.5 \mathrm{wt} \%$ and $10.0 \mathrm{wt} \%$ experiments, the recovery was approximately 20 percentage points higher than from the baseline experiment. Note that these recoveries are compared for experiments performed over the same length of time. The major reason for such a large increase in production from solvent addition is the pressure support. The injected solvent quickly pressurized the model to the target pressure and started to produce the oil, whereas in the steam only case, it took much longer before the target production pressure was achieved.

Figure 4.7 compares the cumulative produced oil from the experiments with carbon dioxide addition with that from the steam only experiment. Quantitatively, the $5.0 \mathrm{wt} \%$ addition experiment produced $508 \mathrm{~mL}$ of oil. This was 2.45 times more oil production than in the baseline experiment. On the other hand, the $7.5 \mathrm{wt} \%$ and $10.0 \mathrm{wt} \%$ experiments produced 429 and $407 \mathrm{~mL}$ of oil. Compared to the $5.0 \mathrm{wt} \%$ experiment, this oil production was lower by $15.6 \%$ for the 7.5 $\mathrm{wt} \%$ experiment and by $19.9 \%$ for the $10.0 \mathrm{wt} \%$ experiment. 
To ensure that the aforementioned results were valid, all three experiments were repeated and the reproducibility of the experiments was found to be within an error of less than $0.5 \%$. The standard deviation in the produced oil volume for $5.0 \mathrm{wt} \%$ carbon dioxide test is shown in Figure 4.7

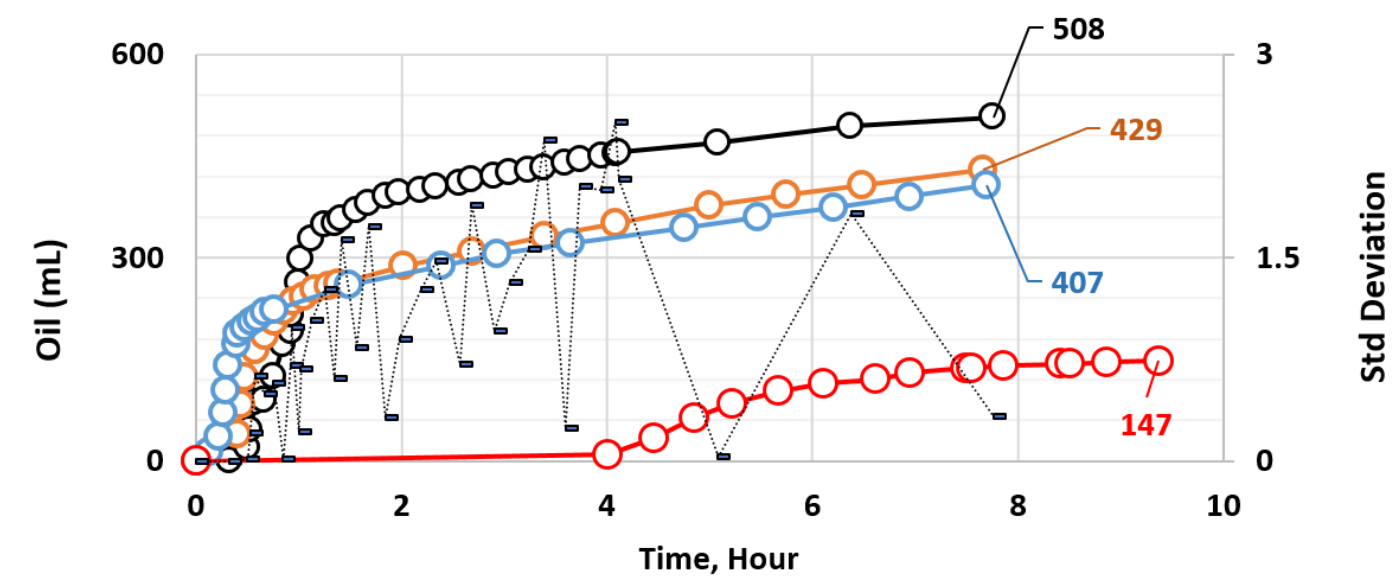

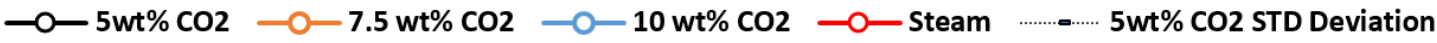

Figure 4.7 Produced oil comparison of steam only with steam plus $\mathrm{CO}_{2}$ experiments

In order to seek a functional relationship between the injected carbon dioxide concentration and cumulative ultimate oil recovery, these two parameters were plotted with cumulative oil recovery on the y-axis, and were curve fitted with a second-order polynomial. As shown in Figure 4.8, a curve was obtained with a clear decreasing trend in the oil production with increased concentration of the carbon dioxide in the injected stream.

The equation below presents the relationship between the carbon dioxide concentration (in wt $\%$ ) with oil recovery from a hybrid SAGD process:

\%OOIP Recovery $=0.0031$ Conc $_{\mathrm{CO}_{2}}^{2}-0.0614$ Conc $_{\mathrm{CO}_{2}}+0.5938$ 


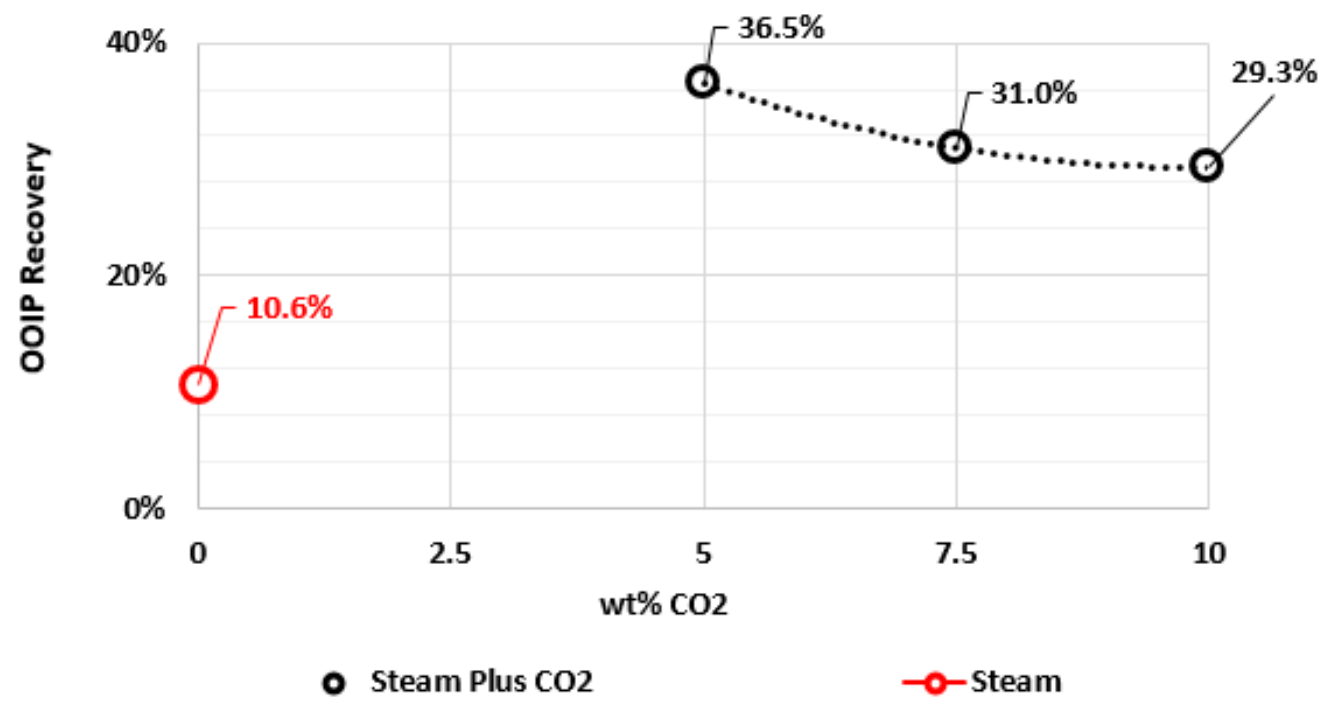

Figure $4.8 \%$ OOIP recovery versus $\mathrm{CO}_{2}$ concentration

Figure 4.9 presents the comparison of differential pressure $(\Delta \mathrm{P})$ among these experiments. The $5.0 \mathrm{wt} \%$ experiment showed the highest $\Delta \mathrm{P}$, especially during the earlier time of the run. On the other hand, the $10.0 \mathrm{wt} \%$ experiment showed the highest $\Delta \mathrm{P}$ at the later time of the run. The baseline experiment with steam showed the lowest $\Delta \mathrm{P}$ among all these experiments. An increased $\Delta \mathrm{P}$ is the sign of flow of viscous fluid between the wells.

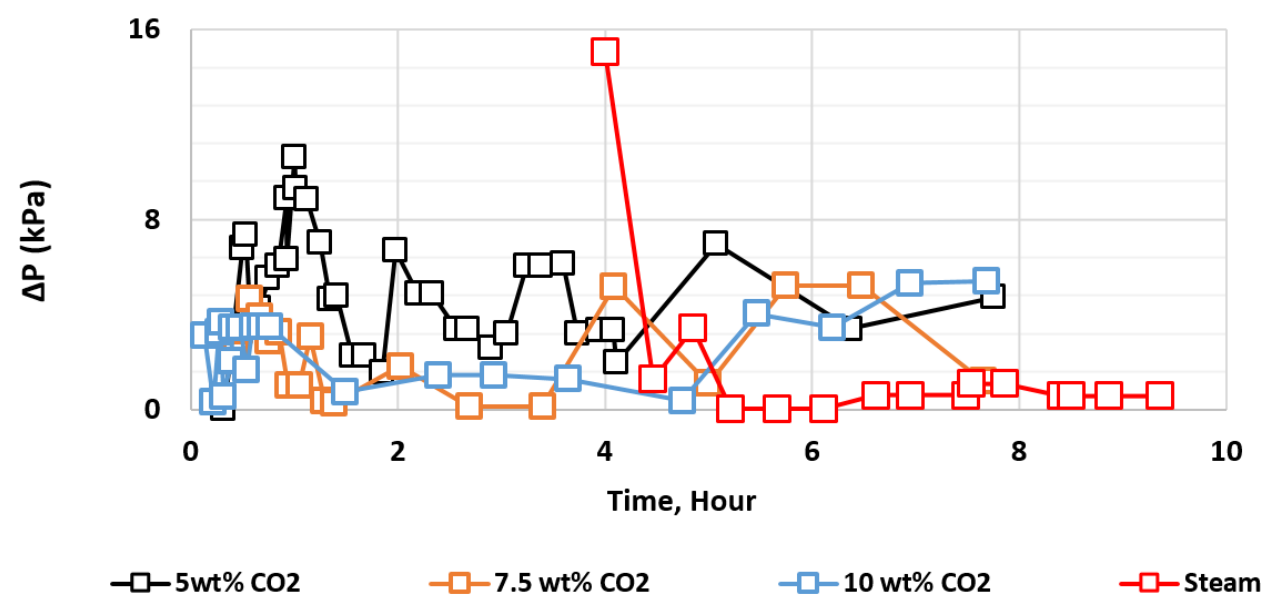

Figure 4.9 Differential pressure comparison of steam only with steam plus $\mathrm{CO}_{2}$ experiments

Figure 4.10 shows the injected and produced water during the experiment with steam plus $5.0 \mathrm{wt} \%$ carbon dioxide. Steam with a cold-water equivalent (CWE) of $892 \mathrm{~mL}$ was injected during this 
experiment. Approximately $18 \%$ of the injected water remained in the model, with $733 \mathrm{~mL}$ being produced. As can be observed from the figure, during the earlier time, the water production curve is not smooth, unlike that obtained after about 1.75 hours into the process. This is almost the same time when there was a significant drop in the slope of the produced oil curve.

Figure 4.11 shows the injected and produced water during the experiment with steam plus $7.5 \mathrm{wt} \%$ carbon dioxide. Steam with a cold-water equivalent (CWE) of $880 \mathrm{~mL}$ was injected during this experiment. Approximately $12 \%$ of the injected water remained in the model, with $776 \mathrm{~mL}$ being produced.

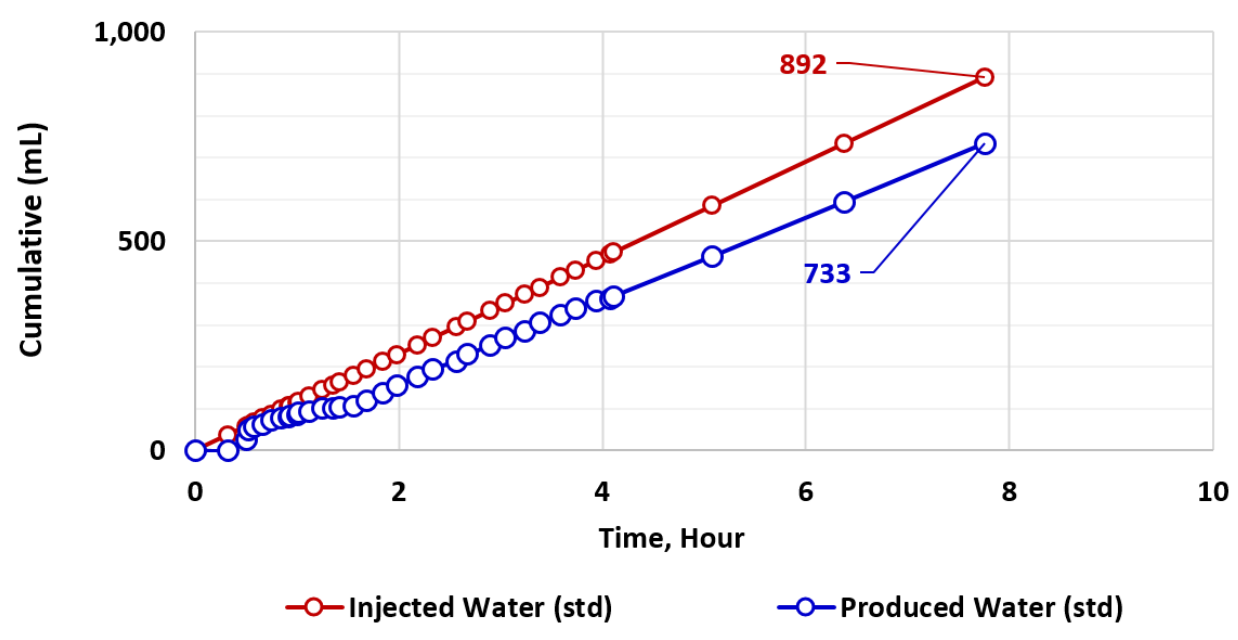

Figure 4.10 Steam plus $5.0 \mathrm{wt} \% \mathrm{CO}_{2}$ - injected and produced water

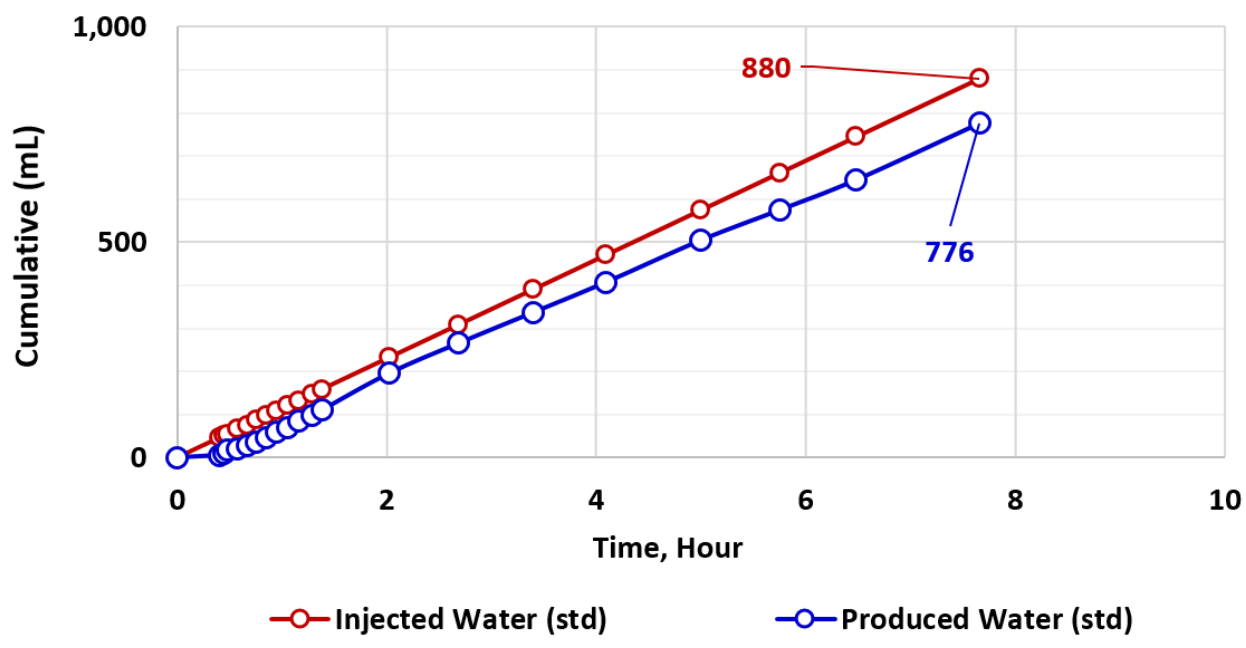

Figure 4.11 Steam plus 7.5 wt $\% \mathrm{CO}_{2}$ - injected and produced water 
Figure 4.12 shows the injected and produced water during the experiment with steam plus 10.0 wt\% carbon dioxide. Steam with a cold-water equivalent (CWE) of $884 \mathrm{~mL}$ was injected during this experiment. Approximately $10 \%$ of the injected water remained in the model, with $798 \mathrm{~mL}$ being produced. A clear trend was observed in reduction in the water retention with an increased concentration of carbon dioxide in the injected stream.

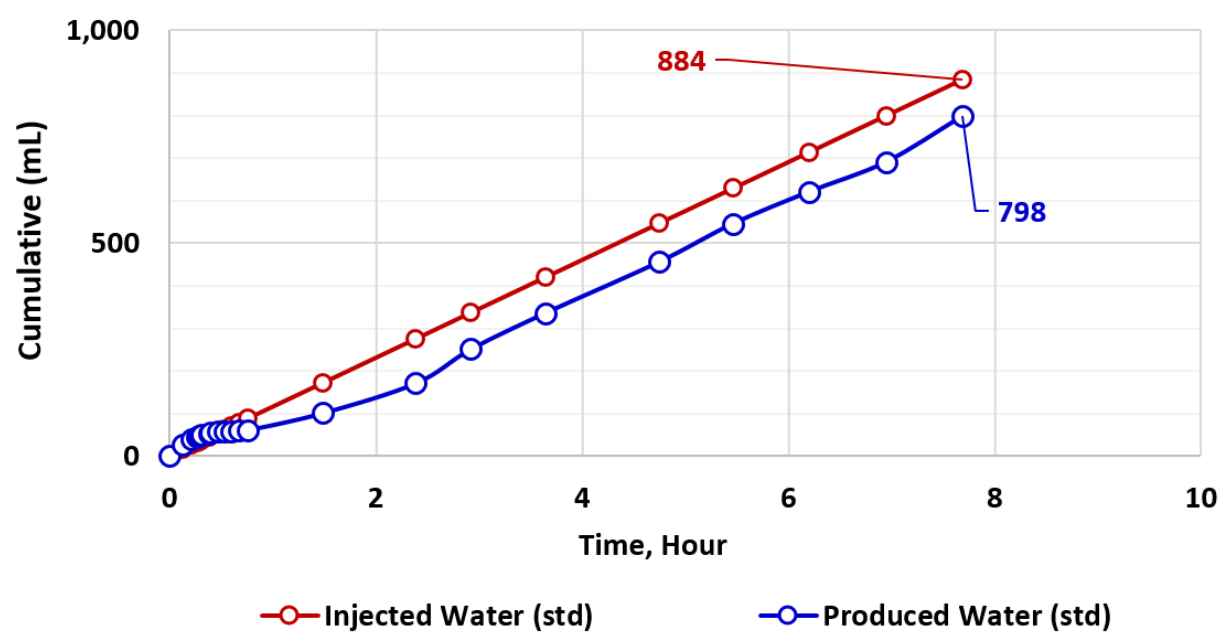

Figure 4.12 Steam plus $10.0 \mathrm{wt} \% \mathrm{CO}_{2}$ - injected and produced water

Figure 4.13 shows the injected and produced gas during the experiment with steam plus $5.0 \mathrm{wt} \%$ carbon dioxide. A total of 24 standard (std) litres of gas was injected. Approximately $8 \%$ of the injected gas remained in the model, with 22 std litres being produced. There was a delay in oil production, primarily due to pressurizing of the model to the set production pressure of $1.45 \mathrm{MPa}$, gauge. Furthermore, compared to the time when oil started to produce-within the first 30 minutes of injection-it took longer for the gas to start producing, after one hour into injection. This indicates that, at first, the mobile oil between the wells was being pushed out and then gas channeled through between the injection and production wells.

Figure 4.14 shows the injected and produced gas during the experiment with steam plus $7.5 \mathrm{wt} \%$ carbon dioxide. A total of $36 \mathrm{std}$ litres of gas was injected. Approximately the same amount of gas was produced, with hardly any gas retention within the model. Similar to the $5.0 \mathrm{wt} \%$ experiment, it took longer for the gas to be produced than for the oil. However, the gap between oil and gas production was smaller than in the $5.0 \mathrm{wt} \%$ carbon dioxide experiment. In the $7.5 \mathrm{wt} \%$ experiment, 
oil started to produce within the first 25 minutes, whereas the gas started to produce after 34 minutes into injection. This indicates that gas broke through earlier between injection and production wells than in the $5.0 \mathrm{wt} \%$ experiment

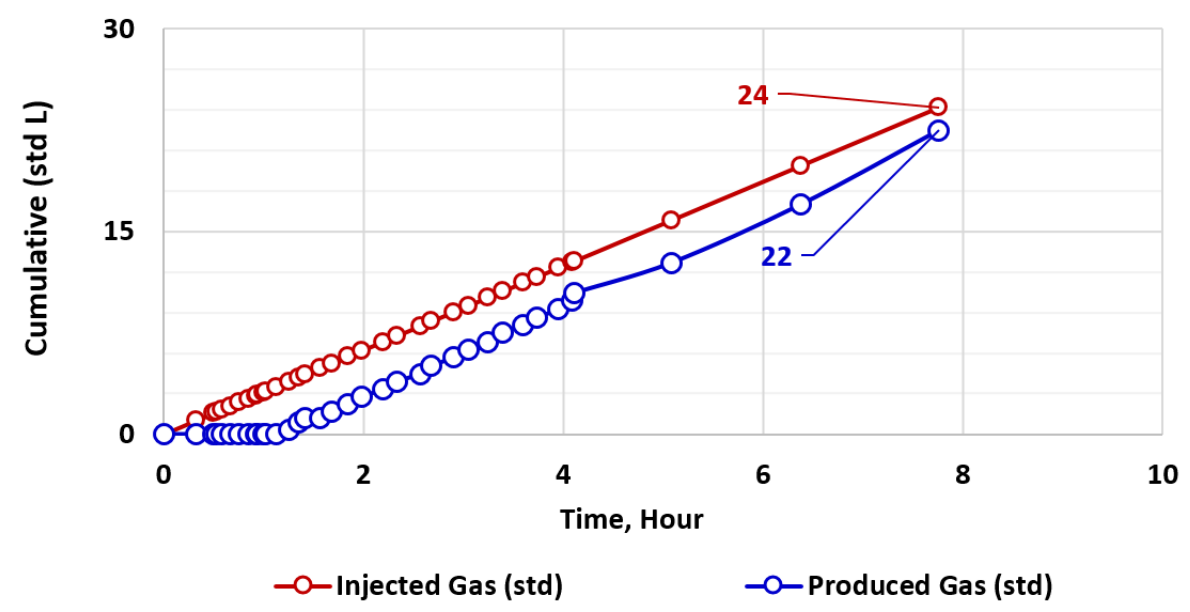

Figure 4.13 Steam plus $5.0 \mathrm{wt} \% \mathrm{CO}_{2}-$ injected and produced gas

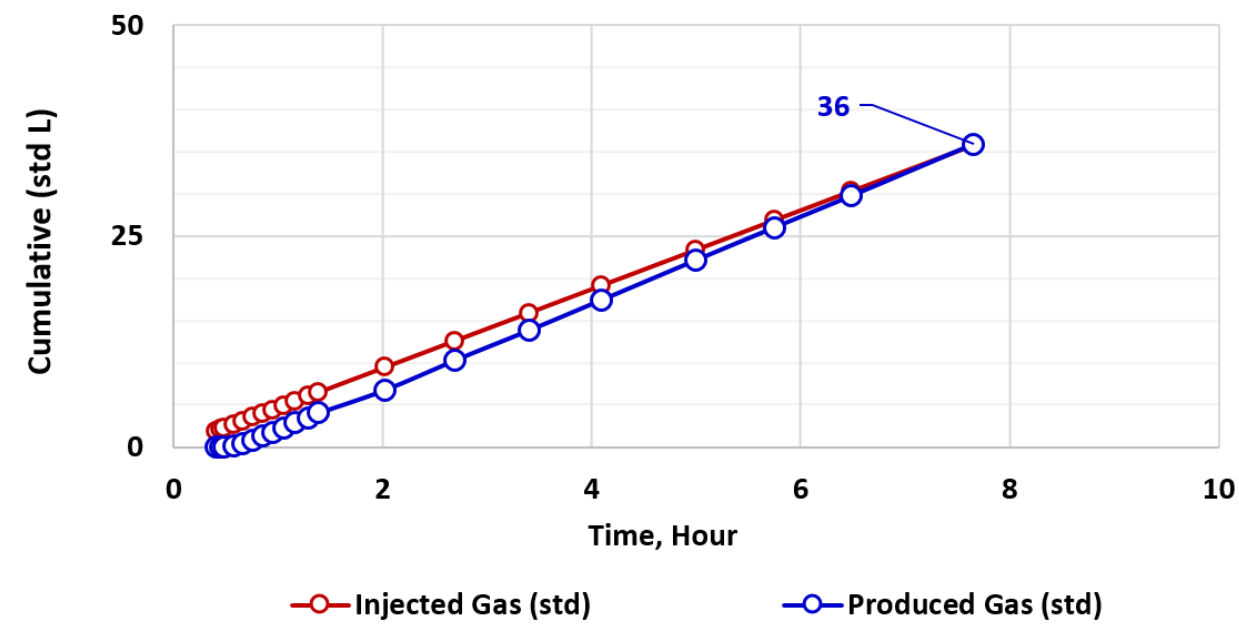

Figure 4.14 Steam plus $7.5 \mathrm{wt} \% \mathrm{CO}_{2}-$ injected and produced gas

Figure 4.15 shows the injected and produced gas during the experiment with steam plus $10.0 \mathrm{wt} \%$ carbon dioxide. A total of $47.5 \mathrm{std}$ litres of gas was injected. Similar to the $7.5 \mathrm{wt} \%$ experiment, the volume of produced gas was very close to the injected gas volume, suggesting no solvent retention in the model, despite the increased concentration of carbon dioxide in the injected stream. Similar to the other two experiments, the time at which the gas started to produce was a bit longer 
compared to the time when oil started to produce. During this experiment, it took approximately 7 minutes for the oil to start producing, whereas it took approximately 23 minutes for the gas to start producing. The highest concentration of carbon dioxide in the injected stream resulted in the quickest breakthrough of the gas between the injection and production wells. This could contribute to lowering of the cumulative oil production that occurred with increased gas concentration in the injected stream.

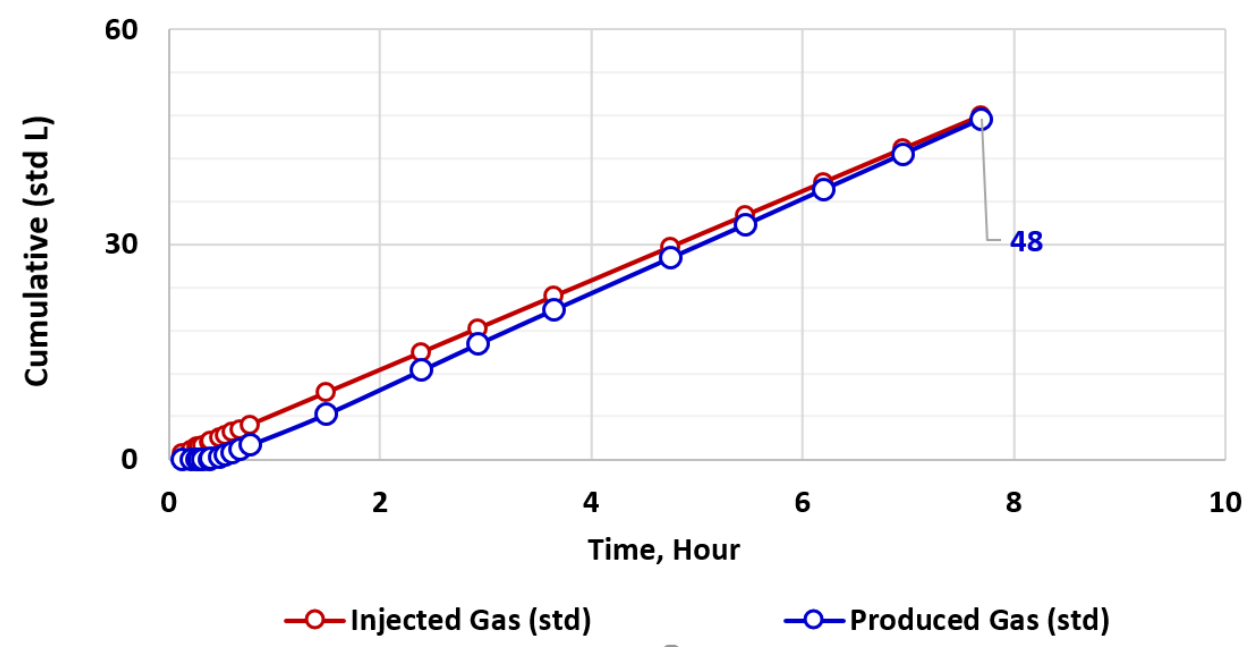

Figure 4.15 Steam plus $10.0 \mathrm{wt} \% \mathrm{CO}_{2}$ - injected and produced gas

\subsection{Steam Plus Propane Injection Experiments}

This section presents the comparison of produced fluids, i.e. produced oil, water and gas; and pressures during the experiments in which propane was injected with steam. The objective here is to investigate the performance of hybrid SAGD by varying the propane concentration in the injected stream and compare the results with the baseline experiment in which only steam was injected.

Figure 4.16 compares the \%OOIP recovery from the experiments with propane addition with the steam only experiment. Note that the model packing, preheating and injection schedule were fairly consistent among all these experiments. 


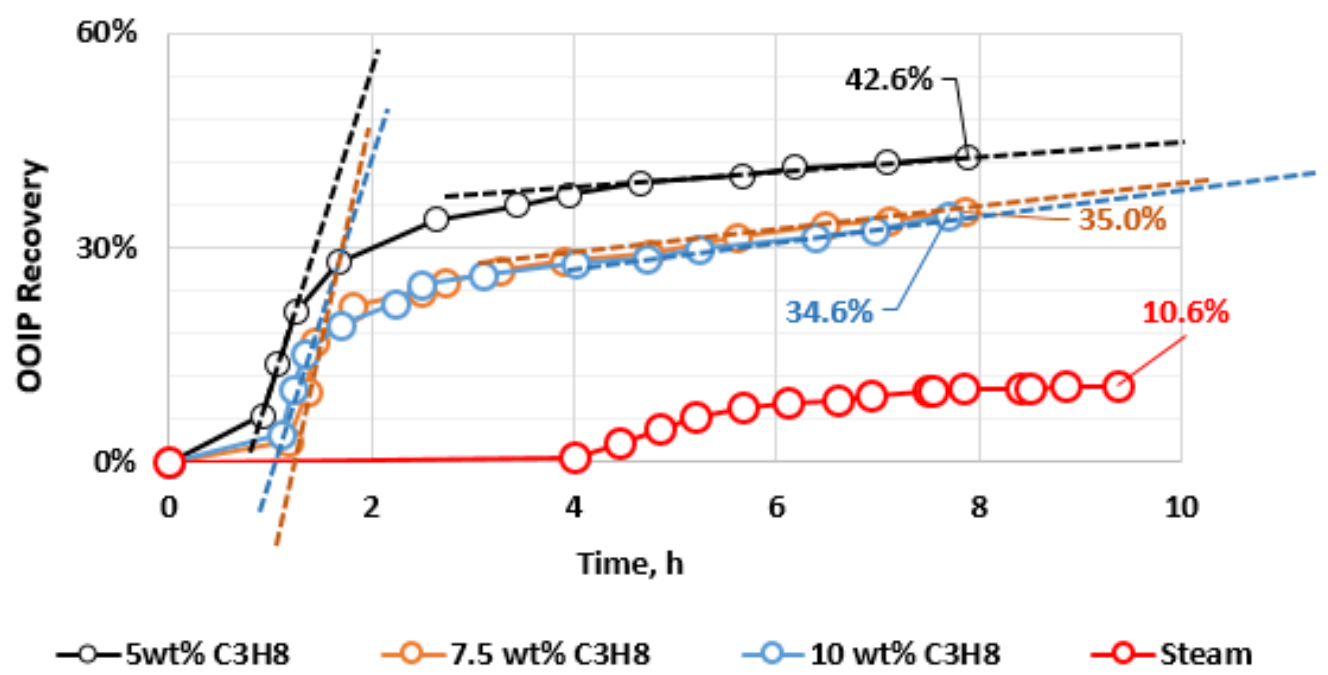

Figure 4.16 Oil recovery comparison of steam only with steam plus $\mathrm{C}_{3} \mathrm{H}_{8}$ experiments

As in the carbon dioxide addition experiments, the ultimate oil recovery decreased with an increase in propane concentration in the injected stream. However; the recoveries from these experiments were higher than those from the carbon dioxide addition experiments. This is due to the higher solubility of propane in oil compared to carbon dioxide. At the experimental conditions of $195^{\circ} \mathrm{C}$ and 1.45 MPa gauge, propane is three times more soluble than carbon dioxide. Thus, with propane, the oil viscosity with dissolved propane would be about $3 \mathrm{cP}$, compared to about $6 \mathrm{cP}$ with carbon dioxide (calculated using the viscosity correlation developed by Freitag (2008). This additional reduction in oil viscosity can result in improved oil recovery.

Note that the molecular weight of both carbon dioxide and propane is almost the same. Thus, the concentration of these two solvents in the injected stream is the same on both the mole\% and mass \% basis.

To further differentiate the effect of propane concentration in the injected stream, the slopes of production curves are indicated with dotted lines in Figure 4.16. As can be observed from the figure, during the initial production period, the slope of the production curve for the highest propane concentration is slightly steeper than for the other two concentrations. Likewise, in the later times, the slopes are slightly steeper for a higher propane concentration in the injected stream. Note that a steeper slope of production curve corresponds to an increased oil production rate. 
As explained for carbon dioxide in Section 4.2, the lower oil production from a higher propane concentration in the injected stream could be attributed to the gas relative permeability effect induced from larger gas volumes.

Compared to the baseline experiment with steam only, all the experiments with propane addition produced substantial volumes of oil. The recovery from the run with $5.0 \mathrm{wt} \%$ propane addition was approximately 32 percentage points higher than that of the baseline experiment. Similarly, from the $7.5 \mathrm{wt} \%$ and $10.0 \mathrm{wt} \%$ experiments, the recoveries were approximately 24 percentage points higher than from the baseline experiment. Note that these recoveries are compared on experiments performed over the same length of time. Similar to carbon dioxide, propane also demonstrated pressure support and helped in pressurizing the reservoir much faster than steam only.

Figure 4.17 compares the cumulative produced oil from the steam plus propane experiments with that of the steam only experiment. Quantitatively, the $5.0 \mathrm{wt} \%$ addition experiment produced 592 $\mathrm{mL}$ of oil. This was 4.05 times more oil production than in the baseline experiment. On the other hand, compared to the $5.0 \mathrm{wt} \%$ run, the $7.5 \mathrm{wt} \%$ and $10.0 \mathrm{wt} \%$ experiments produced $18.0 \%$ less oil (485 $\mathrm{mL})$ and $18.2 \%$ less oil $(484 \mathrm{~mL})$, respectively.

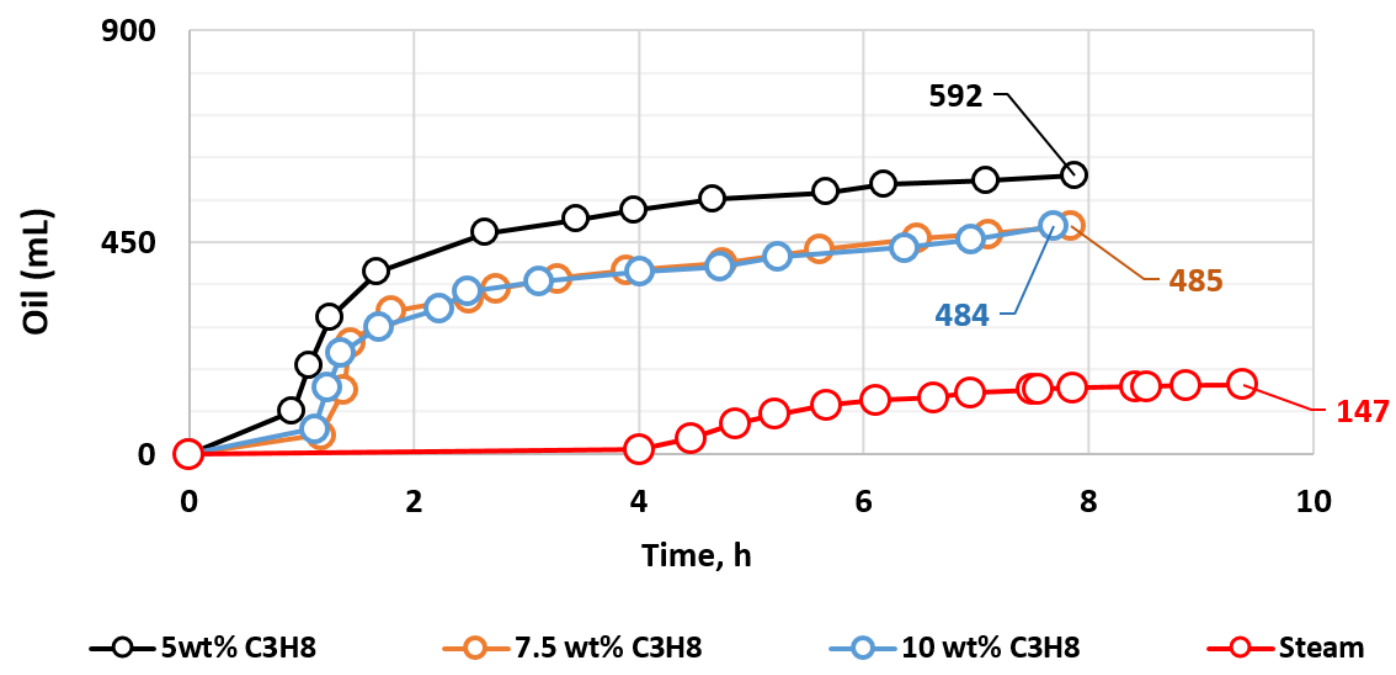

Figure 4.17 Produced oil comparison of steam only with steam plus $\mathrm{C}_{3} \mathrm{H}_{8}$ experiments 
In order to seek a functional relationship between the injected propane concentration and cumulative ultimate oil recovery, these two parameters were plotted with cumulative oil recovery on the y-axis, and were curve fitted with a second-order polynomial. As shown in Figure 4.18, a curve was obtained with a clear decreasing trend in the oil production with increased concentration of propane in the injected stream.

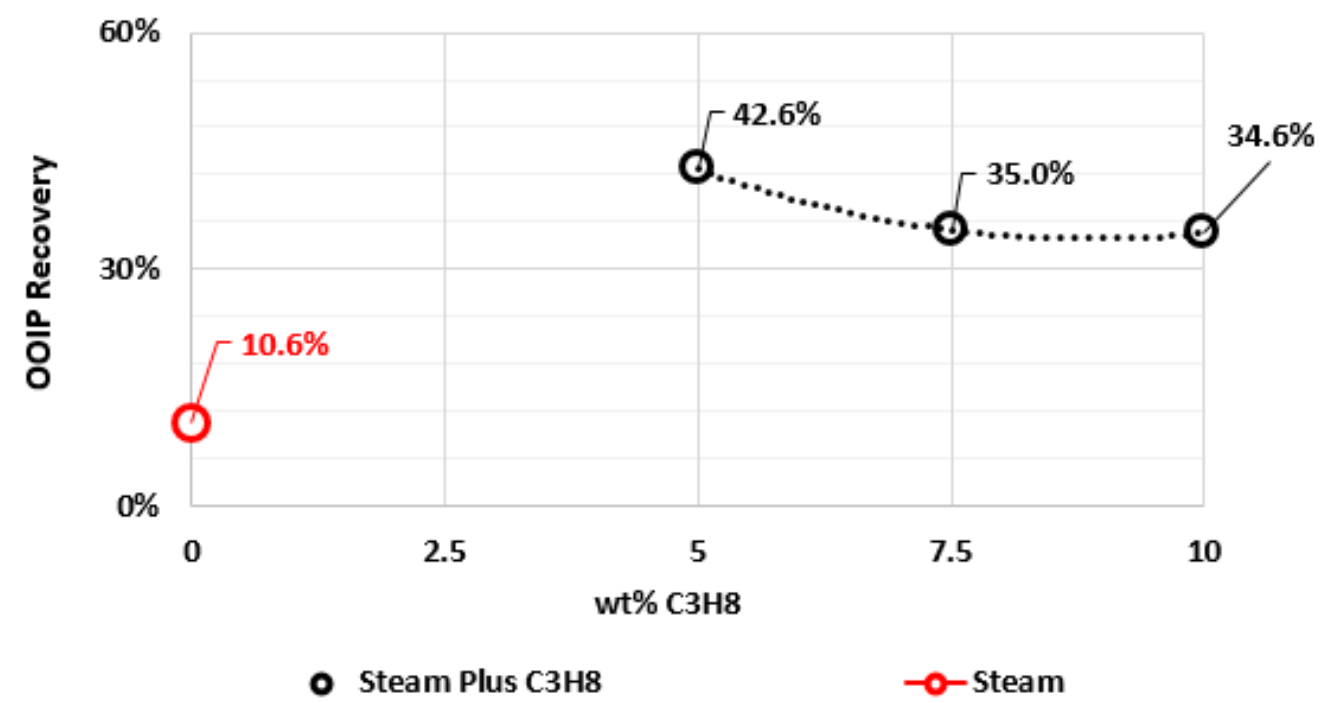

Figure 4.18\%OOIP recovery versus $\mathrm{C}_{3} \mathrm{H}_{8}$ concentration

The equation below presents the relationship between the propane concentration (in wt $\%$ ) and oil recovery from a hybrid SAGD process:

\%OOIP Recovery $=0.0058$ Conc $_{\mathrm{C}_{3} \mathrm{H}_{8}}^{2}-0.1037 \operatorname{Conc}_{\mathrm{C}_{3} \mathrm{H}_{8}}+0.7988$

Figure 4.19 presents the comparison of differential pressure $(\Delta \mathrm{P})$ among these experiments. Overall, the $\Delta \mathrm{P}$ values of the propane experiments were slightly lower than those of the carbon dioxide addition experiments. This could be explained by the higher solubility of propane than of carbon dioxide at the run conditions. Thus, the propane-diluted oil would be less viscous than the oil diluted with carbon dioxide. 


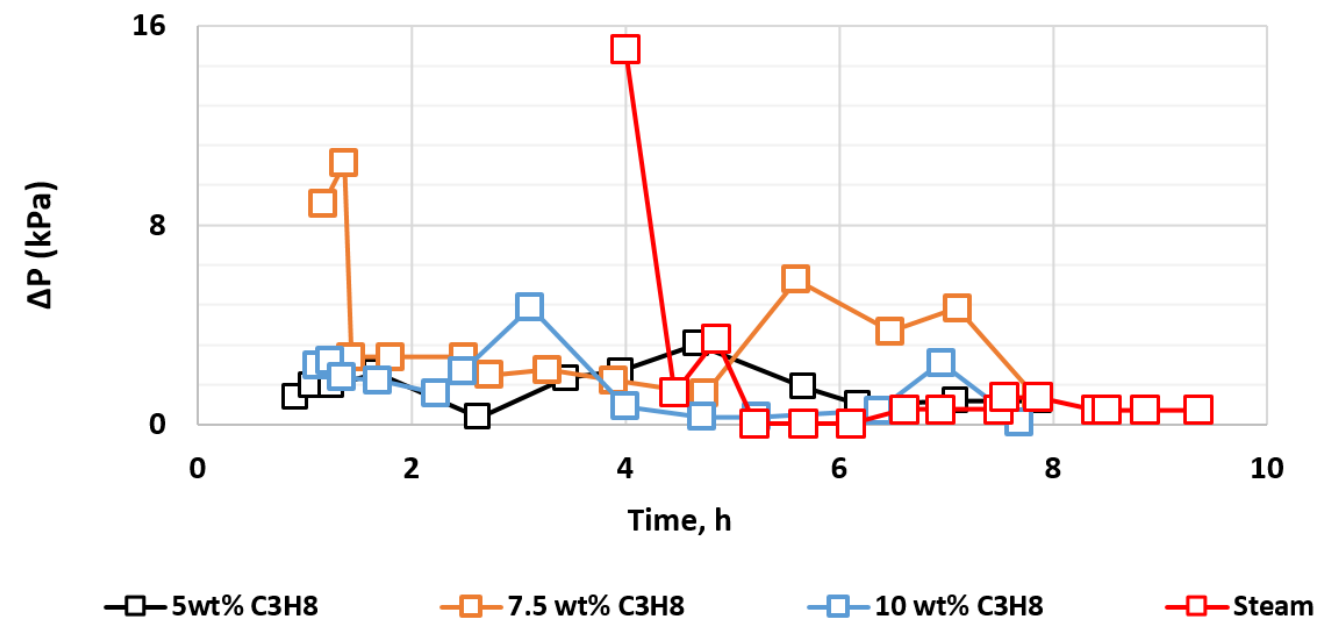

Figure 4.19 $\Delta \mathrm{P}$ comparison of steam only with steam plus $\mathrm{C}_{3} \mathrm{H}_{8}$ experiments

Figure 4.20 shows the injected and produced water during the experiment with steam plus $5.0 \mathrm{wt} \%$ propane. Steam with a cold-water equivalent of $906 \mathrm{~mL}$ was injected during this experiment. Approximately $18 \%$ of the injected water remained in the model, with $739 \mathrm{~mL}$ being produced. This quantity of water retention was similar to what was observed during the $5.0 \mathrm{wt} \%$ carbon dioxide experiment.

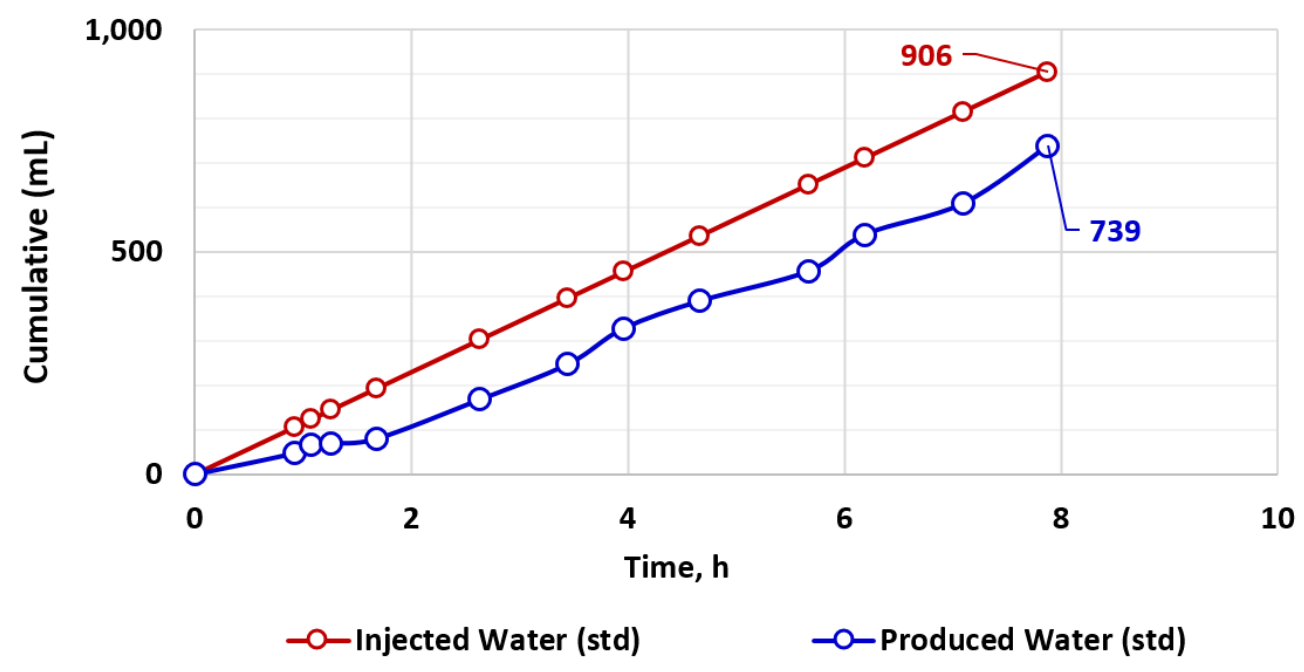

Figure 4.20 Steam plus $5.0 \mathrm{wt} \% \mathrm{C}_{3} \mathrm{H}_{8}$ - injected and produced water

Figure 4.21 shows the injected and produced water during the experiment with steam plus 7.5 wt $\%$ propane. Steam with a cold-water equivalent of $902 \mathrm{~mL}$ was injected during this experiment. 
Approximately $13 \%$ of the injected water remained in the model, with $787 \mathrm{~mL}$ being produced. Again, the volume of the water left inside the model was similar to that in the $7.5 \mathrm{wt} \%$ carbon dioxide experiment.

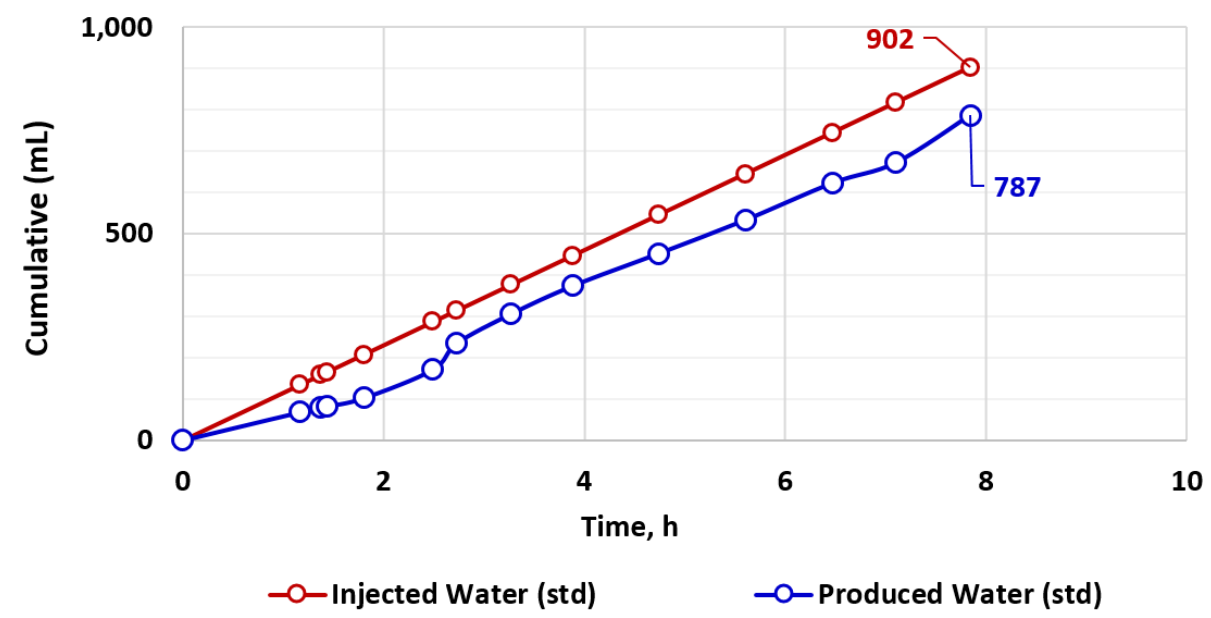

Figure 4.21 Steam plus $7.5 \mathrm{wt} \% \mathrm{C}_{3} \mathrm{H}_{8}$ - injected and produced water

Figure 4.22 shows the injected and produced water during the experiment with steam plus $10.0 \mathrm{wt} \%$ propane. Steam with a cold-water equivalent of $884 \mathrm{~mL}$ was injected during this experiment. Approximately $10 \%$ of the injected water remained in the model, with $794 \mathrm{~mL}$ being produced. A clear trend was observed in reduction in the water retention with increased concentration of propane in the injected stream.

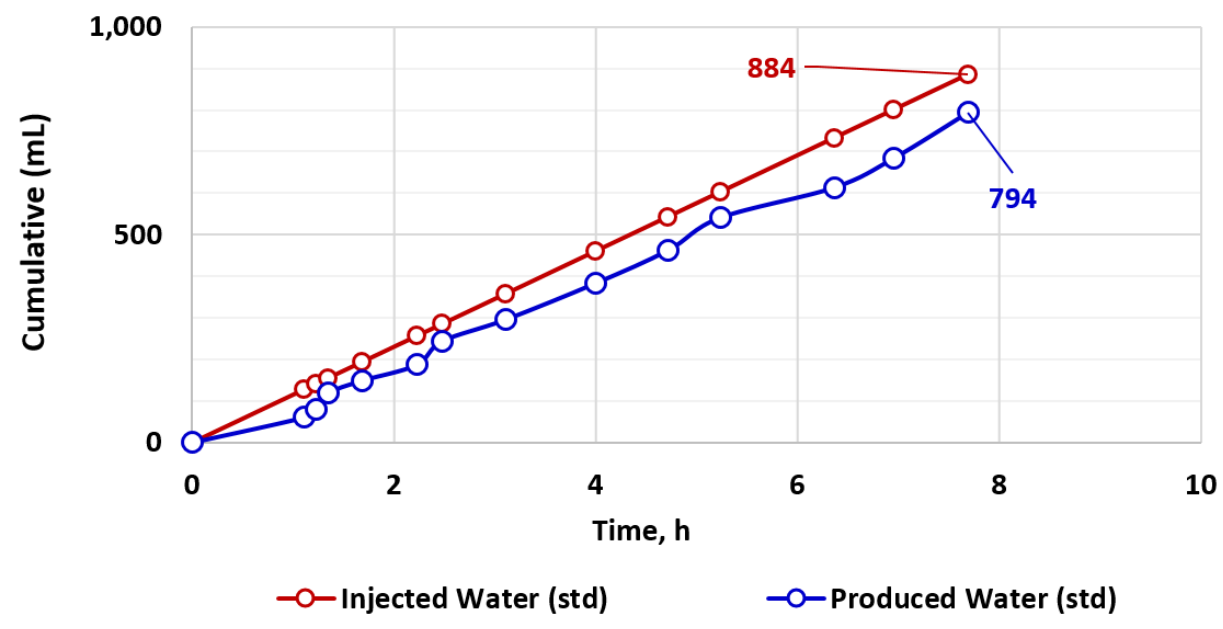

Figure 4.22 Steam plus $10.0 \mathrm{wt} \% \mathrm{C}_{3} \mathrm{H}_{8}$ - injected and produced water 
Figure 4.23 shows the injected and produced gas during the experiment with steam plus $5.0 \mathrm{wt} \%$ propane. A total of 24.1 std litres of gas was injected. Approximately $12 \%$ of the injected gas remained in the model, with 21.1 std litres being produced. The amount of gas retention was higher than in the $5.0 \mathrm{wt} \%$ carbon dioxide experiment, primarily because of the higher solubility of the propane that results in more gas being trapped in the model. Compared to the oil, it took longer for the gas to start producing. During the first 55 minutes of injection, approximately $90 \mathrm{~mL}$ of oil was produced, whereas the gas started to produce after 75 minutes of injection.

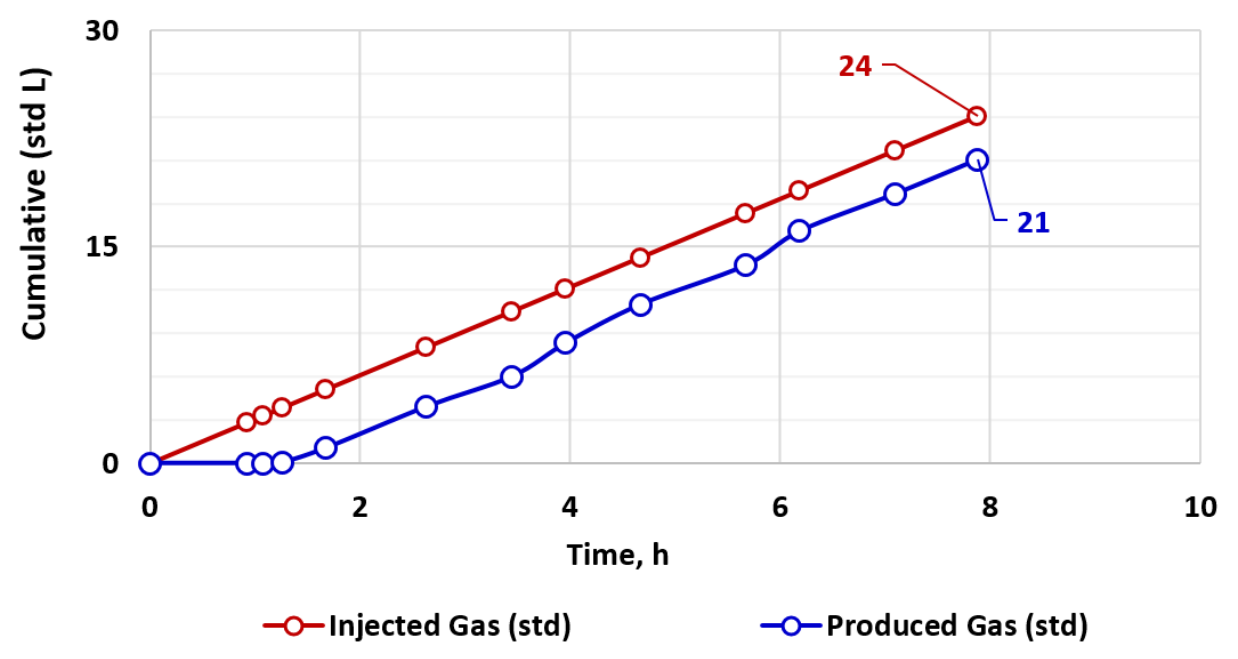

Figure 4.23 Steam plus $5.0 \mathrm{wt} \% \mathrm{C}_{3} \mathrm{H}_{8}$ - injected and produced gas

Figure 4.24 shows the injected and produced gas during the experiment with steam plus $7.5 \mathrm{wt} \%$ propane. A total of 36.6 std litres of gas was injected. Unlike in the carbon dioxide experiment, the produced gas was approximately $11 \%$ less than the injected gas. Only 32.4 litres of gas was recovered. Compared to the time at which oil started to produce, a delay was observed in the start of gas production. During the first 70 minutes of injection, approximately $41 \mathrm{~mL}$ of oil was produced, whereas gas started to produce after 82 minutes of injection.

Figure 4.25 shows the injected and produced gas during the experiment with steam plus $10.0 \mathrm{wt} \%$ propane. A total of 47.0 std litres of gas was injected, of which only 40.3 litres was recovered. This corresponds to approximately $14.2 \%$ solvent retention. These experiments showed that the higher the propane concentration in the injected stream, the higher the solvent retention will be. The gas breakthrough time was found to be even longer than for the $7.5 \mathrm{wt} \%$ propane experiment. During 
the first 66 minutes of injection, approximately $53 \mathrm{~mL}$ of oil was produced, whereas the gas started to produce after approximately 100 minutes. This observation was different than for the carbon dioxide addition experiments, in which increased concentration resulted in early breakthrough times.

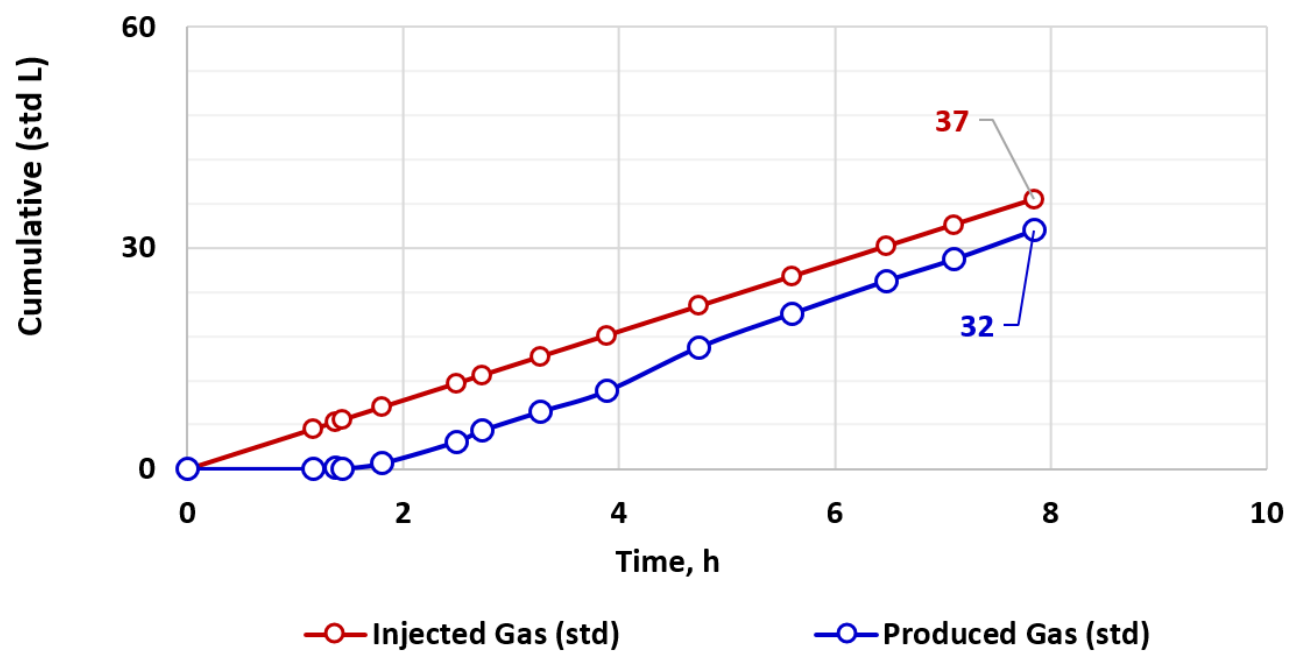

Figure 4.24 Steam plus $7.5 \mathrm{wt} \% \mathrm{C}_{3} \mathrm{H}_{8}$ - injected and produced gas

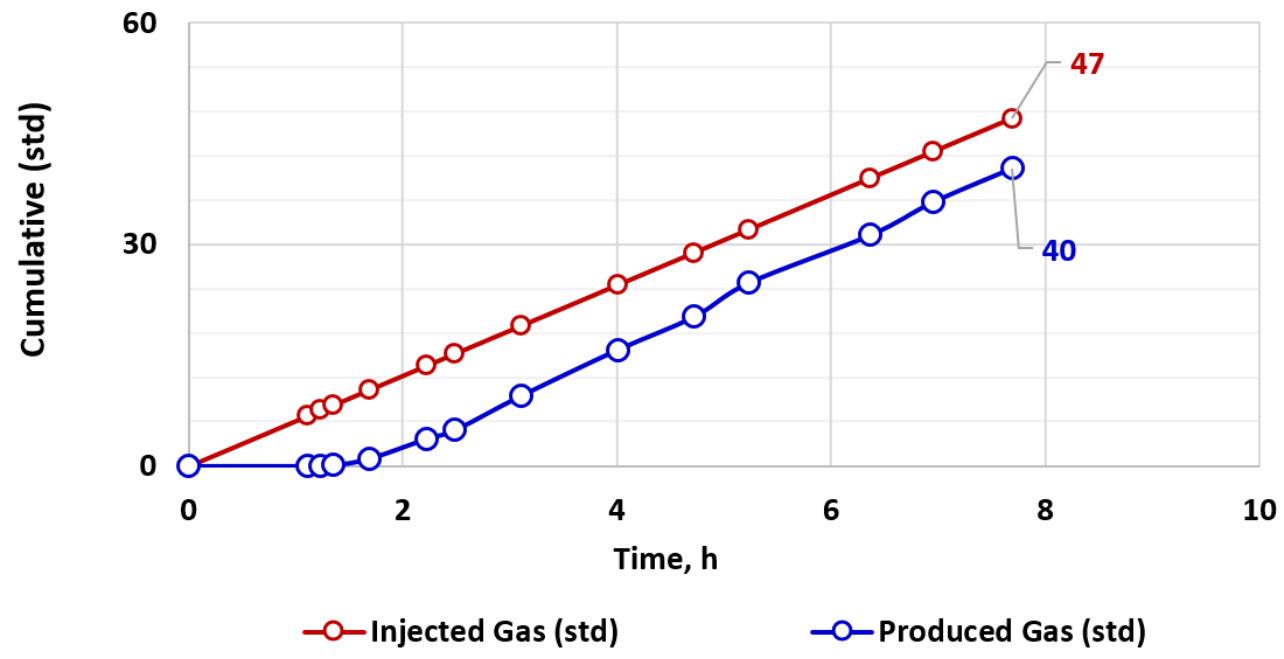

Figure 4.25 Steam plus $10.0 \mathrm{wt} \% \mathrm{C}_{3} \mathrm{H}_{8}-$ injected and produced gas

\subsection{Steam Plus Nitrogen Injection Experiments}

This section presents the comparison of produced fluids, i.e. produced oil, water and gas; and pressures during the experiments in which nitrogen was injected with steam. The objective here is 
to investigate the performance of hybrid SAGD by varying the nitrogen concentration in the injected stream and compare the results with the baseline experiment in which only steam was injected.

Figure 4.26 compares the \%OOIP recovery from the experiments with nitrogen addition with that of the steam only experiment. Note that the model packing, preheating and injection schedule was fairly consistent among all these experiments.

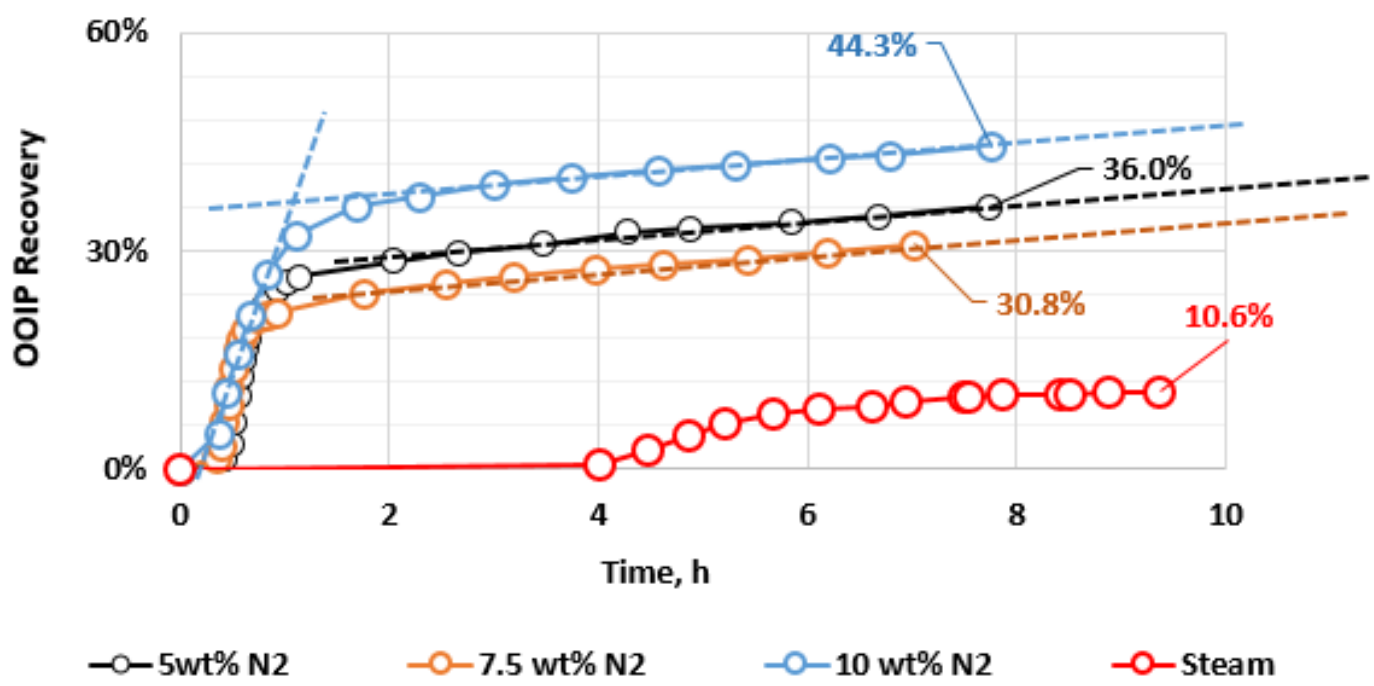

Figure 4.26 Oil recovery comparison of steam only with steam plus $\mathrm{N}_{2}$ experiments

Unlike the carbon dioxide and propane addition experiments, it was the highest concentration among the three concentrations that gave the highest oil recovery: $10.0 \mathrm{wt} \%$ of nitrogen produced $44.3 \%$ OOIP. By contrast, the concentration of $7.5 \mathrm{wt} \%$, interestingly, showed the lowest recovery of $30.8 \%$ OOIP. The recovery from the lowest concentration of $5.0 \mathrm{wt} \%$ was $36.0 \%$ OOIP.

Furthermore, the slopes of the production curves for both the initial production period where oil was being produced at higher rates, and later production periods where oil was being produced at lower rates, were identical for each concentration.

Compared to the baseline experiment with steam only, all the experiments with nitrogen addition produced substantial volumes of oil. The recovery from the $10.0 \mathrm{wt} \%$ nitrogen addition experiment 
was approximately 34 percentage points higher than from the baseline experiment. From the 5.0 $\mathrm{wt} \%$ and $7.5 \mathrm{wt} \%$ experiments, the recovery was approximately 25.4 and 20.2 percentage points, respectively, higher than that of the baseline experiment.

Figure 4.27 compares the cumulative produced oil from the experiments with nitrogen addition with the steam only experiment. Quantitatively, the $5.0 \mathrm{wt} \%$ addition experiment produced 496 $\mathrm{mL}$ of oil, 3.37 times more than in the baseline experiment. In comparison to the $5.0 \mathrm{wt} \%$ run, the $7.5 \mathrm{wt} \%$ run produced $428 \mathrm{~mL}$, or $13.7 \%$ less oil; on the other hand, the $10.0 \mathrm{wt} \%$ experiment produced $616 \mathrm{~mL}$ of oil, or $24.1 \%$ more oil.

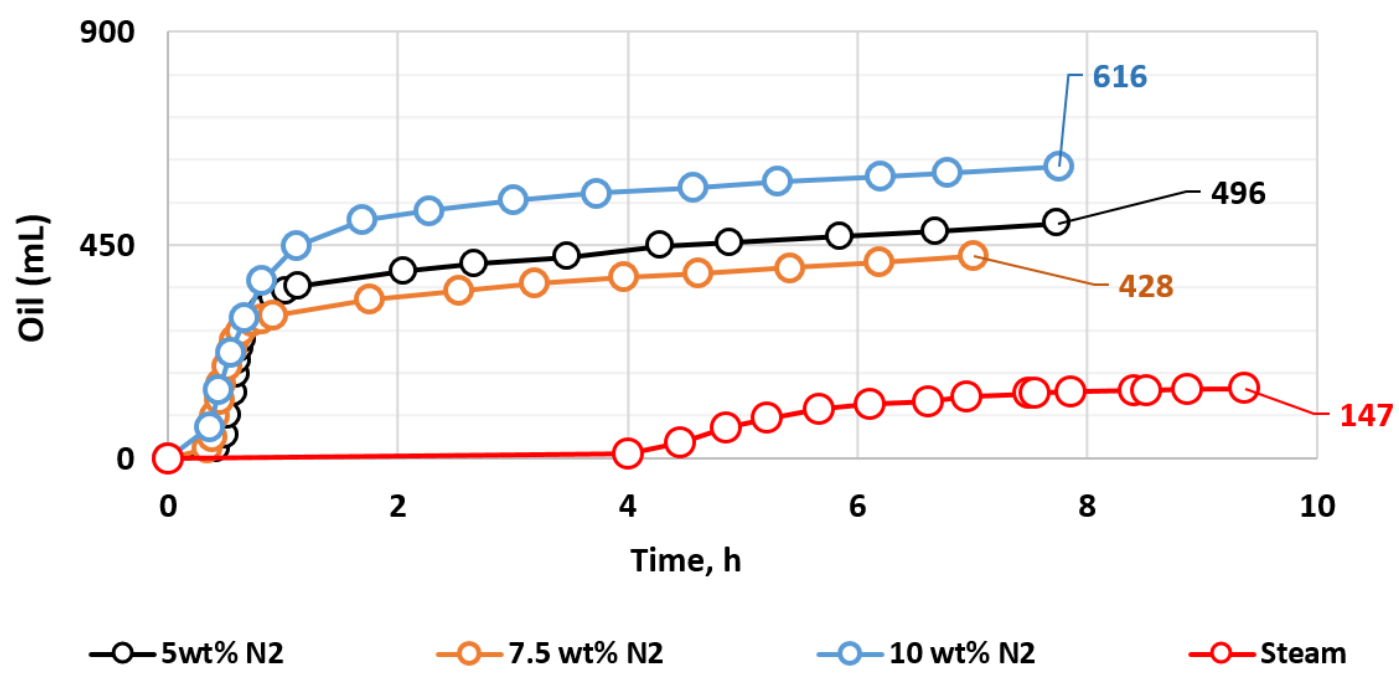

Figure 4.27 Produced oil comparison of steam only with steam plus $\mathrm{N}_{2}$ experiments

In order to seek a functional relationship between the injected nitrogen concentration and cumulative ultimate oil recovery, these two parameters were plotted with cumulative oil recovery on the y-axis, and were curve fitted with a second-order polynomial (Figure 4.28). The equation below presents the relationship between the nitrogen concentration (in wt \%) and oil recovery from a hybrid SAGD process:

\%OOIP Recovery $=0.0149 \operatorname{Conc}_{\mathrm{N}_{2}}^{2}-0.2075 \operatorname{Conc}_{\mathrm{N}_{2}}+1.0242$ 


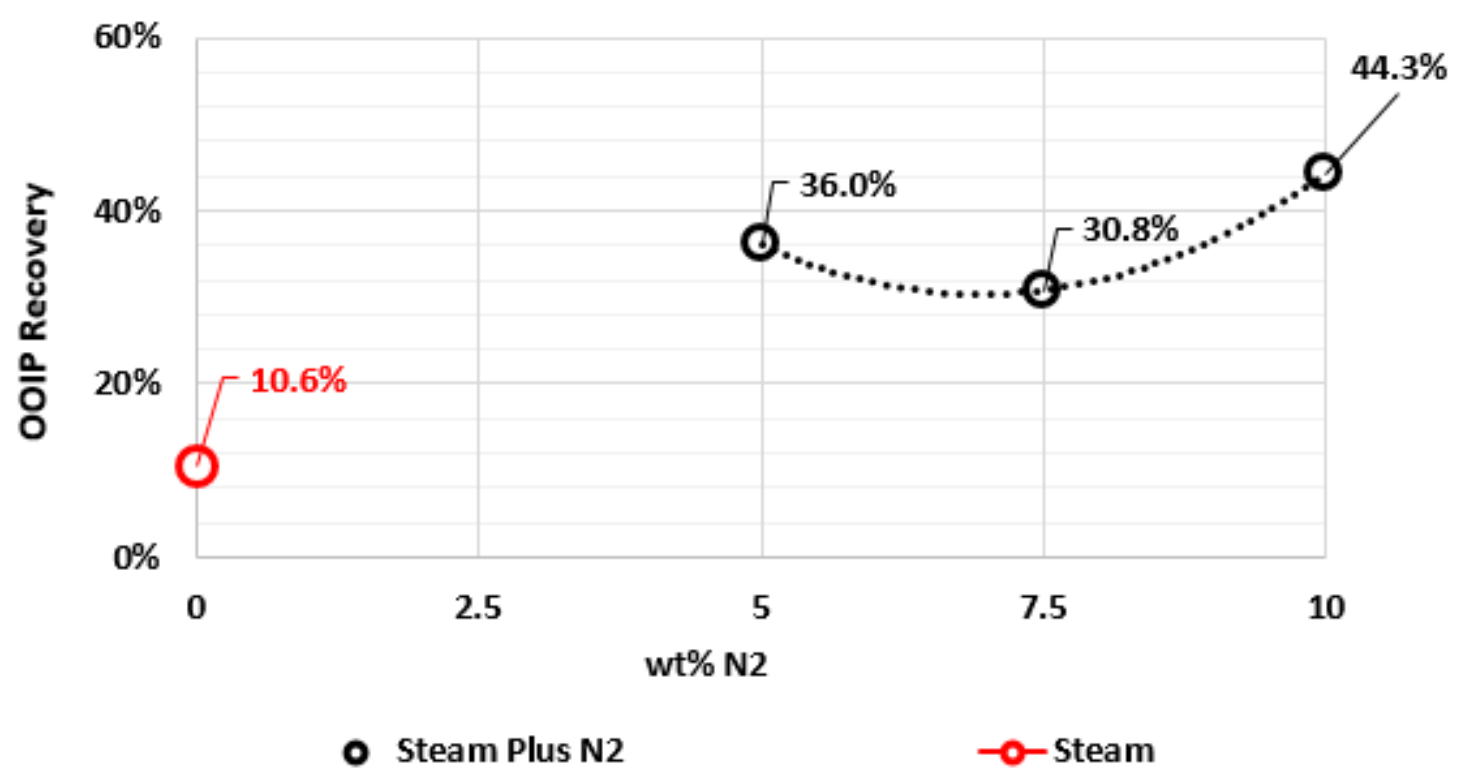

Figure $4.28 \%$ OOIP recovery versus $\mathrm{N}_{2}$ concentration

Figure 4.29 presents the comparison of differential pressure $(\Delta \mathrm{P})$ among these experiments. The $10.0 \mathrm{wt} \%$ run showed the highest $\Delta \mathrm{P}$ for most of the experiment.

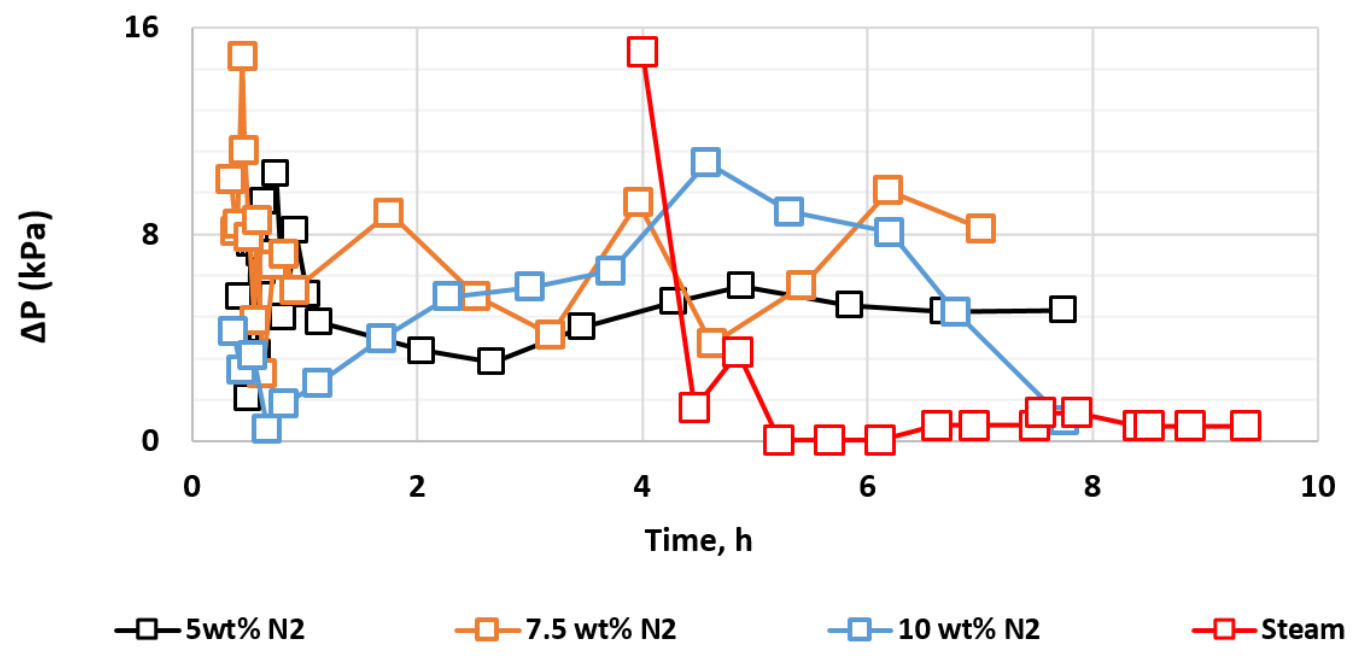

Figure 4.29 $\Delta \mathrm{P}$ comparison of steam only with steam plus $\mathrm{N}_{2}$ experiments

Figure 4.30 shows the injected and produced water during the experiment with steam plus $5.0 \mathrm{wt} \%$ nitrogen. Steam with a cold-water equivalent of $890 \mathrm{~mL}$ was injected during this experiment. 
Approximately $12 \%$ of the injected water remained in the model, with $784 \mathrm{~mL}$ being produced. This quantity of water retention was less than in the previous two experiments with carbon dioxide and propane at $5.0 \mathrm{wt} \%$ concentrations.

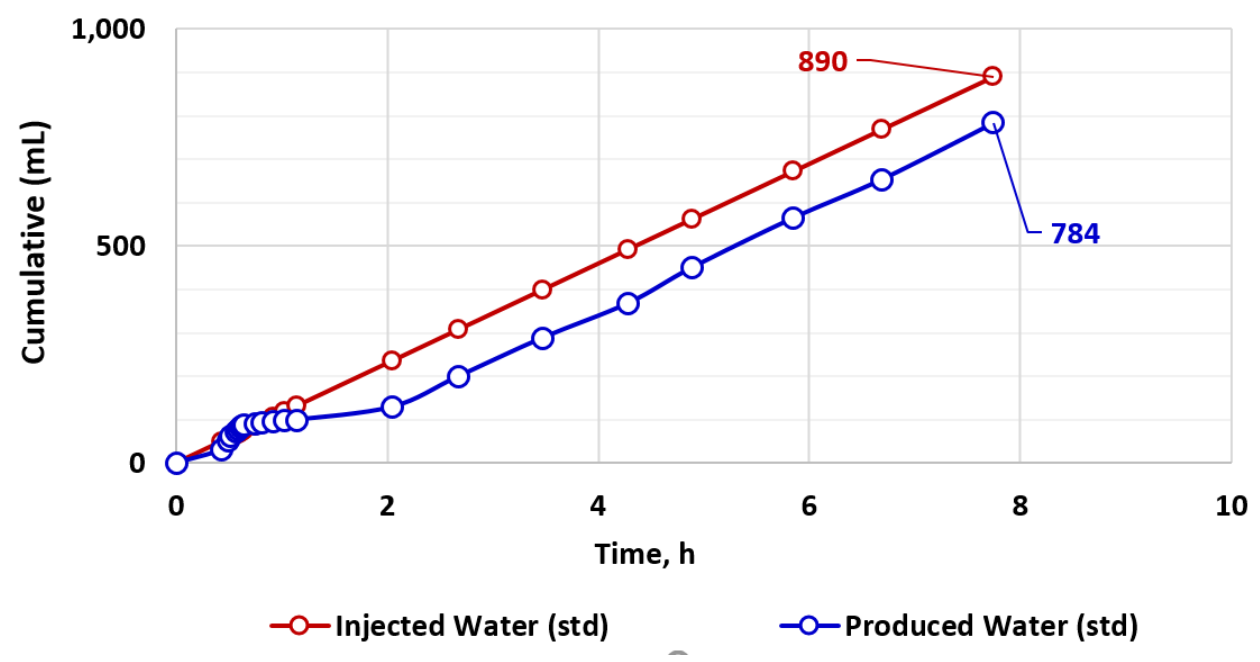

Figure 4.30 Steam plus $5.0 \mathrm{wt} \% \mathrm{~N}_{2}$ - injected and produced water

Figure 4.31 shows the injected and produced water during the experiment with steam plus $7.5 \mathrm{wt} \%$ propane. Steam with a cold-water equivalent of $807 \mathrm{~mL}$ was injected during this experiment. Approximately $5 \%$ of the injected water remained in the model, with $764 \mathrm{~mL}$ being produced. Again, the volume of the water left inside the model was less than in the comparable $7.5 \mathrm{wt} \%$ experiments with carbon dioxide and propane additions.

Figure 4.32 shows the injected and produced water during the experiment with steam plus $10.0 \mathrm{wt} \%$ propane. Steam with a cold-water equivalent of $892 \mathrm{~mL}$ was injected during this experiment. Approximately $9 \%$ of the injected water remained in the model, with $764 \mathrm{~mL}$ being produced.

Figure 4.33 shows the injected and produced gas during the experiment with steam plus $5.0 \mathrm{wt} \%$ nitrogen. A total of 38.1 std litres of gas was injected. Approximately $6 \%$ of the injected gas remained in the model, with 35.9 std litres being produced. The amount of gas retention was lower 
than in the $5.0 \mathrm{wt} \%$ propane experiment. It took longer for the gas to start producing than for the oil. During the first 25 minutes of injection, approximately $20 \mathrm{~mL}$ of oil was produced, whereas the gas started to produce after 34 minutes of injection.

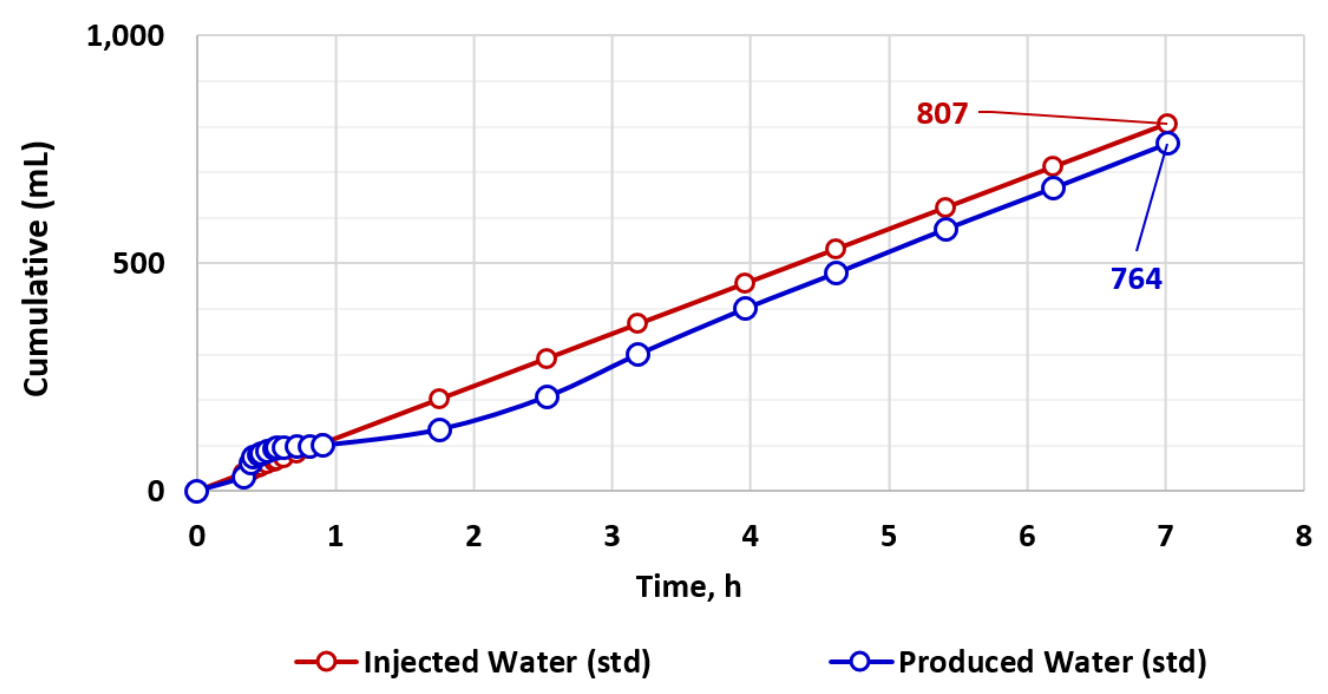

Figure 4.31 Steam plus $7.5 \mathrm{wt} \% \mathrm{~N}_{2}$ - injected and produced water

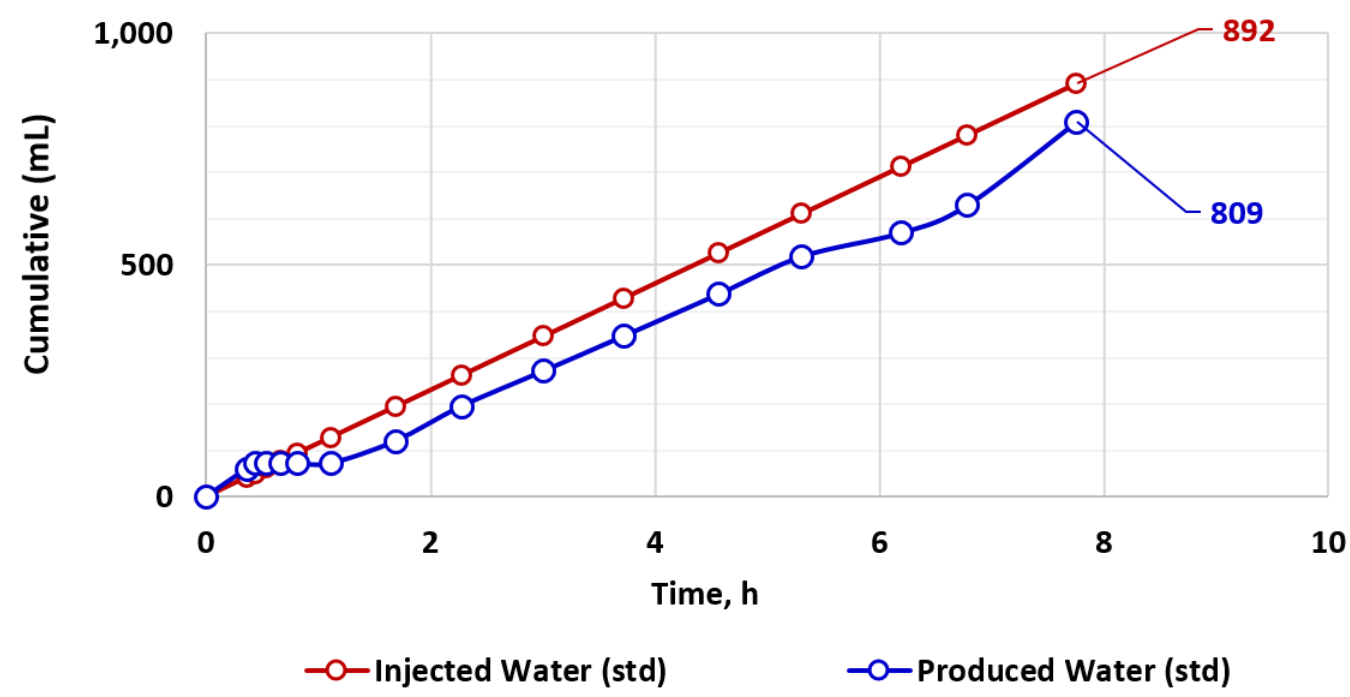

Figure 4.32 Steam plus $10.0 \mathrm{wt} \% \mathrm{~N}_{2}$ - injected and produced water 


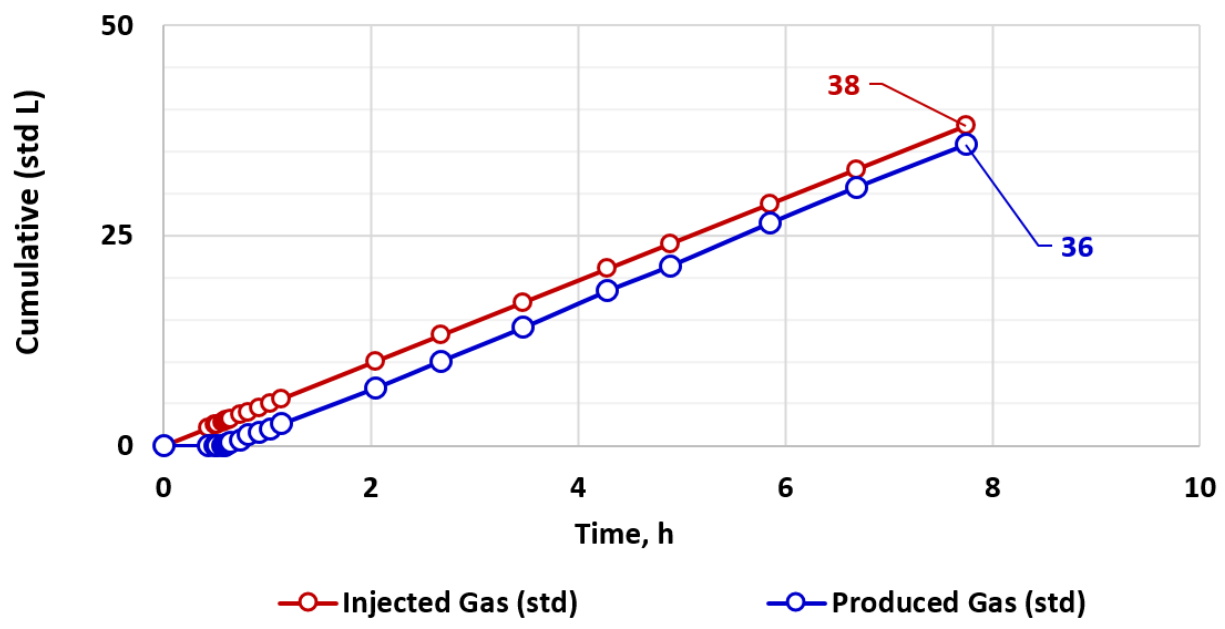

Figure 4.33 Steam plus $5.0 \mathrm{wt} \% \mathrm{~N}_{2}$ - injected and produced gas

Figure 4.34 shows the injected and produced gas during the experiment with steam plus $7.5 \mathrm{wt} \%$ nitrogen. A total of 51.3 std litres of gas was injected. The produced gas - 47.5 litres-was approximately $7 \%$ less than the injected gas. Compared to the time at which oil started to produce, a delay was observed in the start of the gas production. During the first 20 minutes, approximately $20 \mathrm{~mL}$ of oil was produced, whereas gas started to produce after 26 minutes of injection.

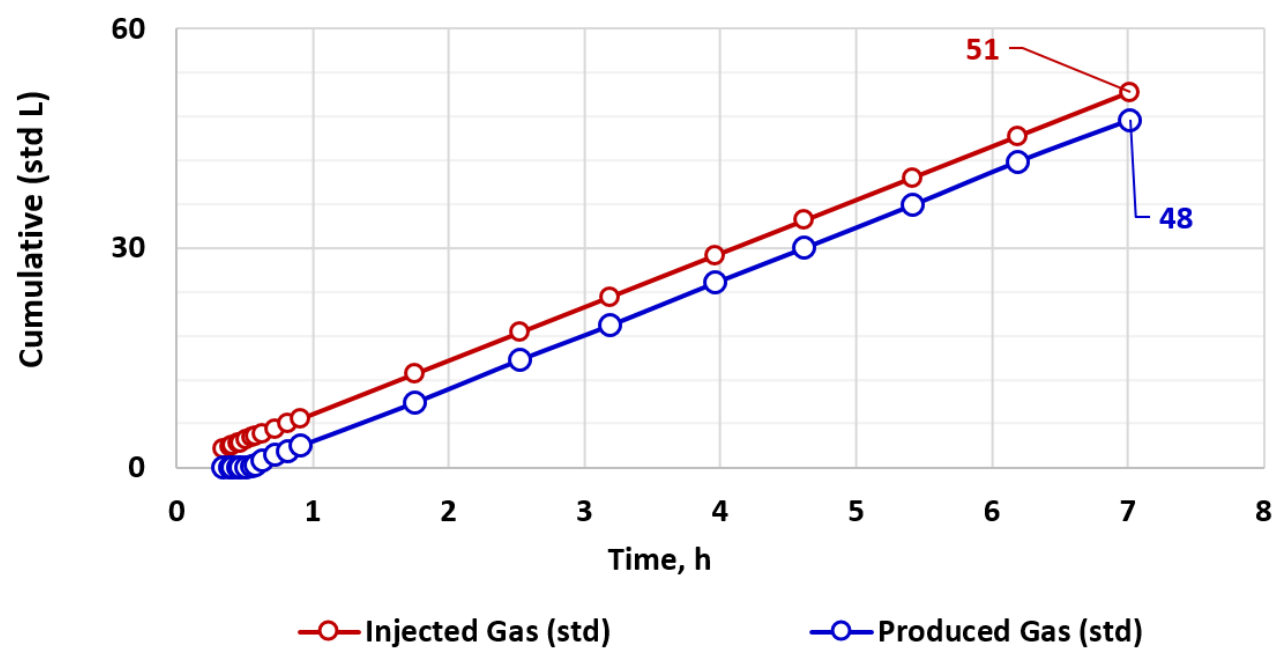

Figure 4.34 Steam plus $7.5 \mathrm{wt} \% \mathrm{~N}_{2}-$ injected and produced gas

Figure 4.35 shows the injected and produced gas during the experiment with steam plus $10.0 \mathrm{wt} \%$ nitrogen. A total of 75.9 std litres of gas was injected; of which only 71.9 litres of gas was 
recovered. This corresponds to approximately $4 \%$ solvent retention. For the highest nitrogen concentration, the gas retention was found to be the lowest. For this experiment, the gas breakthrough occurred at almost the same time as the oil started to produce.

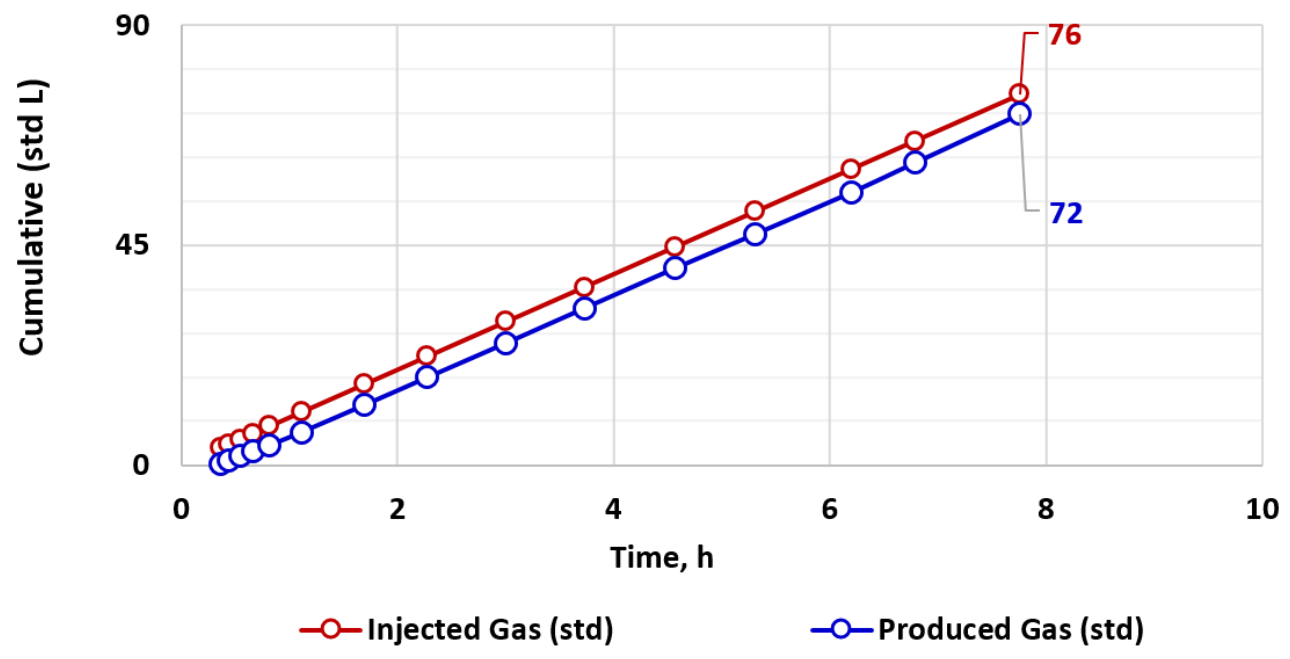

Figure 4.35 Steam plus $10.0 \mathrm{wt} \% \mathrm{~N}_{2}$ - injected and produced gas

\subsection{Steam Plus Methane Injection Experiments}

This section presents the comparison of produced fluids, i.e. produced oil, water and gas; and pressures during the experiments in which methane was injected with steam. The objective here is to investigate the performance of hybrid SAGD by varying the methane concentration in the injected stream and compare the results with the baseline experiment where only steam was injected.

Figure 4.36 compares the \%OOIP recovery from the experiments with methane addition with that of the steam only experiment. Note that the model packing, preheating and injection schedule was consistent among all these experiments.

Again, unlike the carbon dioxide and propane addition experiments, among the three concentrations, the highest concentration of $10.0 \mathrm{wt} \%$ of methane showed the highest oil recovery of 50.7\%. Unlike the nitrogen addition experiments, the lowest concentrations of $5.0 \mathrm{wt} \%$ and 7.5 
$\mathrm{wt} \%$ resulted in identical recoveries. Quantitatively, the $5.0 \mathrm{wt} \%$ and $7.5 \mathrm{wt} \%$ methane addition experiments recovered $38.2 \%$ and $38.0 \%$ OOIP, respectively.

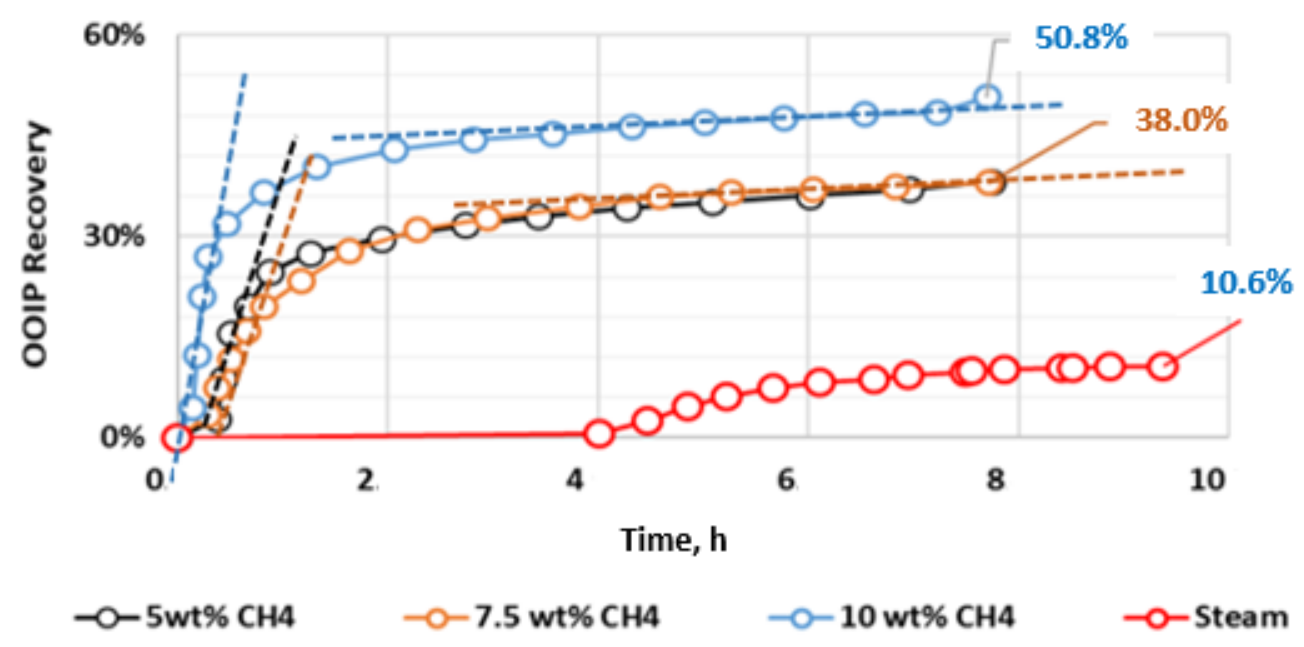

Figure 4.36 Oil recovery comparison of steam only with steam plus $\mathrm{CH}_{4}$ experiments

The slopes of the production curves, as indicated by the dotted lines in Figure 4.36, are identical during the later time of the production when the oil production rate was low. On the other hand, the highest methane concentration resulted in the steepest slope during the early production time.

Compared to the baseline experiment with steam only, all the experiments with methane addition produced substantial volumes of oil. The recovery from the $10.0 \mathrm{wt} \%$ methane addition experiment was approximately 40 percentage points higher than from the baseline experiment. From the 5.0 $\mathrm{wt} \%$ and $7.5 \mathrm{wt} \%$ experiments, the recoveries were approximately 27.6 and 27.4 percentage points, respectively, higher than from the baseline experiment.

Figure 4.37 compares the cumulative produced oil from the experiments with methane addition and from the steam only experiment. Quantitatively, the $5.0 \mathrm{wt} \%$ addition experiment produced $530 \mathrm{~mL}$ of oil — or 3.60 times more oil production than in the baseline experiment. The $7.5 \mathrm{wt} \%$ run produced a similar amount, $537 \mathrm{~mL}$. On the other hand, the $10.0 \mathrm{wt} \%$ experiment produced $705 \mathrm{~L}$ of oil, or $33.0 \%$ more than the $5.0 \mathrm{wt} \%$ methane experiment. 


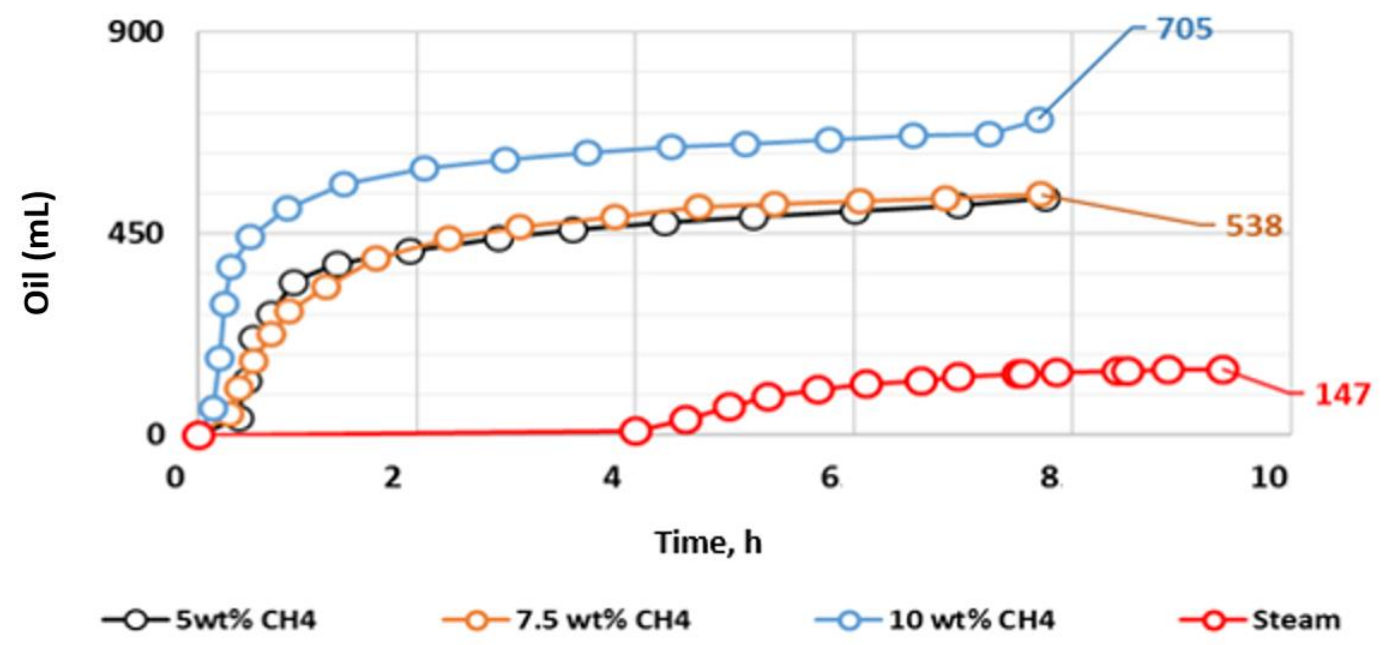

Figure 4.37 Produced oil comparison of steam only with steam plus $\mathrm{CH}_{4}$ experiments

In order to seek a functional relationship between the injected methane concentration and cumulative ultimate oil recovery, these two parameters were plotted with cumulative oil recovery on the y-axis, and were curve fitted with a second-order polynomial (Figure 4.38). The equation below presents the relationship between the methane concentration (in wt\%) with oil recovery from a hybrid SAGD process:

\%OOIP Recovery $=0.0103 \operatorname{Conc}_{\mathrm{CH}_{4}}^{2}-0.1297 \operatorname{Conc}_{\mathrm{CH}_{4}}+0.7722$

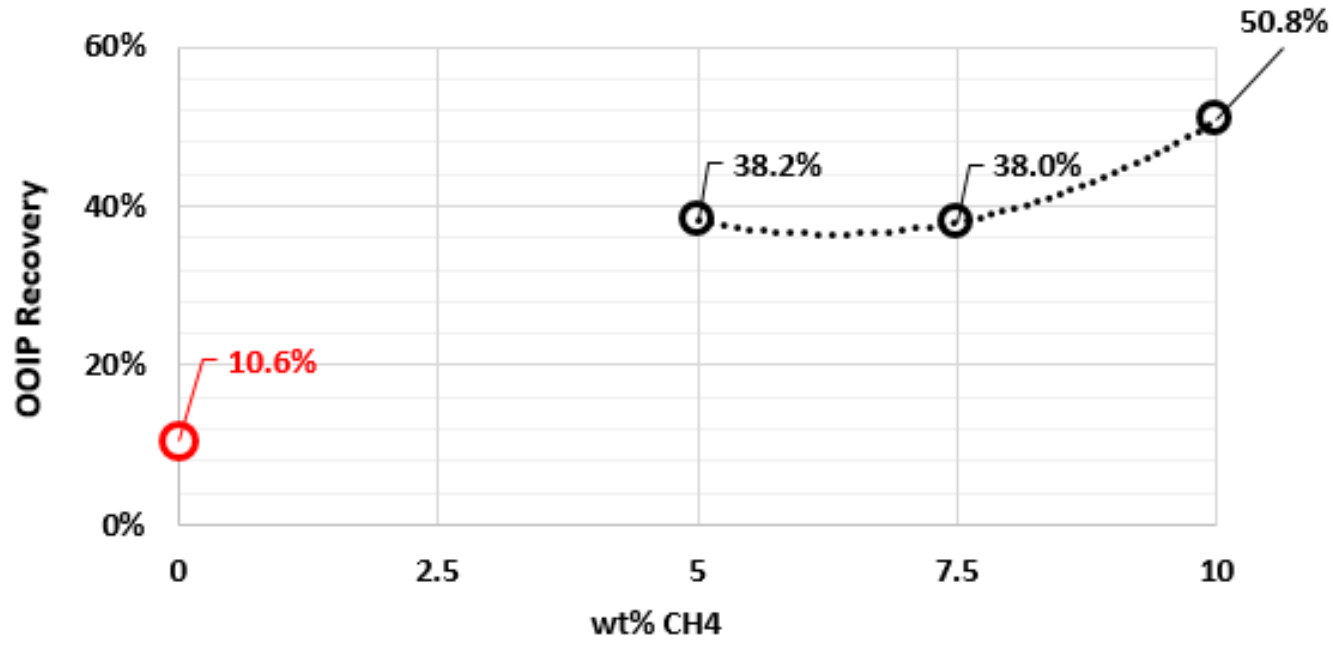

- Steam Plus N2

- - Steam

Figure 4.38\%OOIP recovery versus $\mathrm{CH}_{4}$ concentration 
Figure 4.39 presents the comparison of differential pressure $(\Delta \mathrm{P})$ among these experiments. The $10.0 \mathrm{wt} \%$ experiment showed the highest $\Delta \mathrm{P}$ for most of the run. In fact, the $\Delta \mathrm{P}$ from this experiment was highest among all the experiments performed with different solvents and different concentrations.

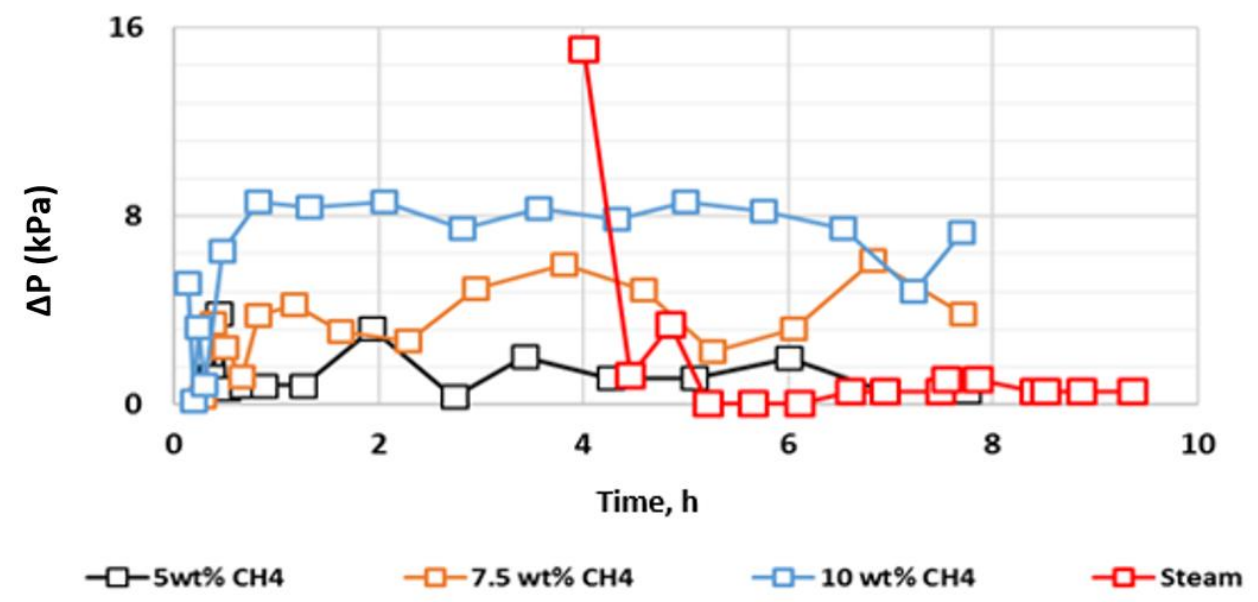

Figure 4.39 $\Delta \mathrm{P}$ comparison of steam only with steam plus $\mathrm{CH}_{4}$ experiments

Figure 4.40 shows the injected and produced water during the experiment with steam plus $5.0 \mathrm{wt} \%$ methane. Steam with a cold-water equivalent of $890 \mathrm{~mL}$ was injected during this experiment. Approximately $3 \%$ of the injected water remained in the model, with $863 \mathrm{~mL}$ being produced. This quantity of water retention was less than in all the previous experiments with carbon dioxide, propane and nitrogen at $5.0 \mathrm{wt} \%$ concentrations.

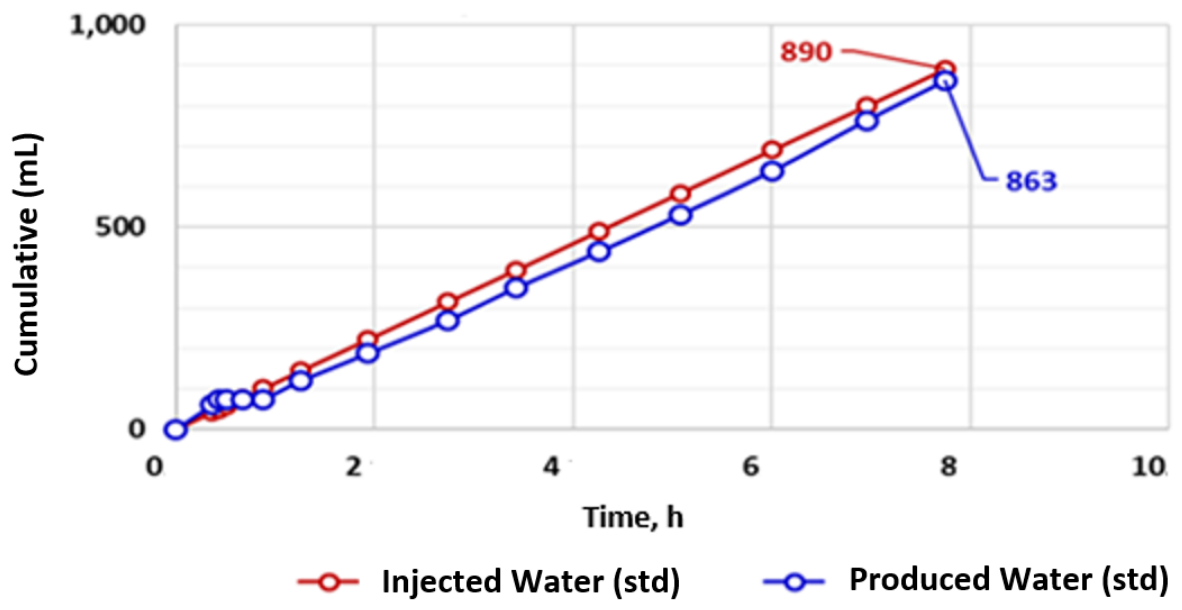

Figure 4.40 Steam plus 5.0 wt\% $\mathrm{CH}_{4}$ - injected and produced water 
Figure 4.41 shows the injected and produced water during the experiment with steam plus $7.5 \mathrm{wt} \%$ methane. Steam with a cold-water equivalent of $887 \mathrm{~mL}$ was injected during this experiment. Approximately $13 \%$ of the injected water remained in the model, with $786 \mathrm{~mL}$ being produced.

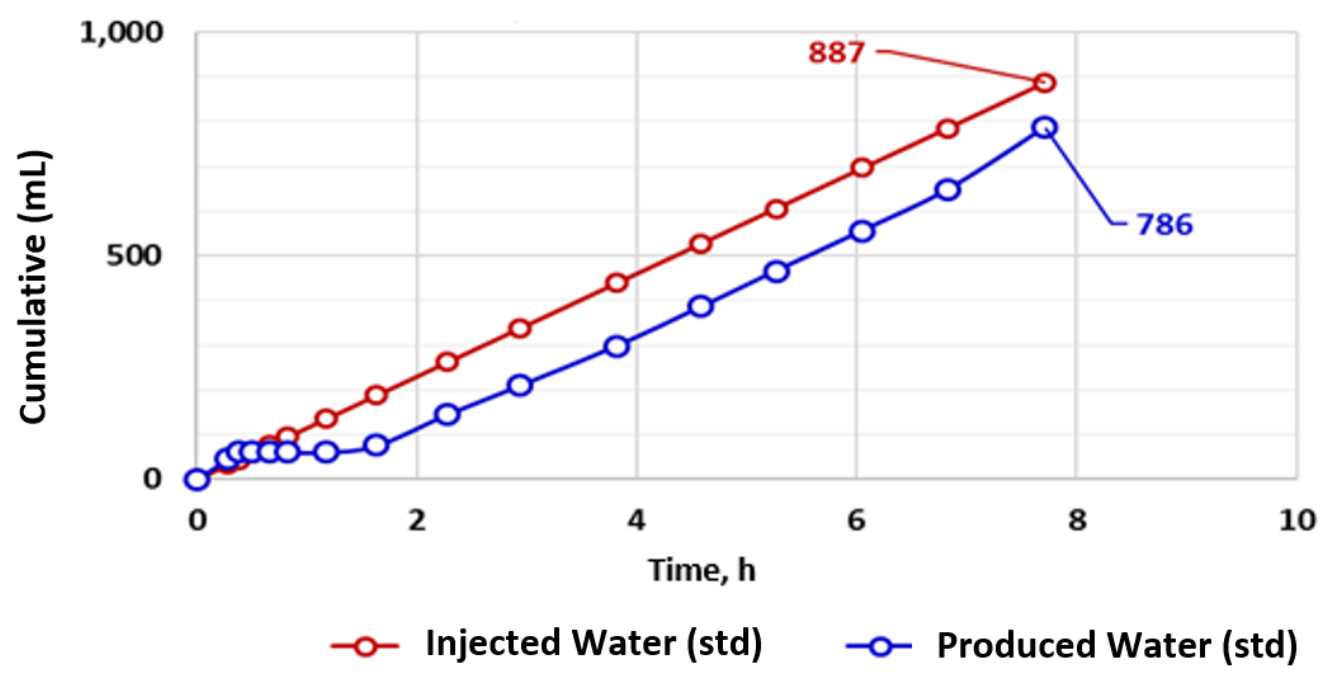

Figure 4.41 Steam plus $7.5 \mathrm{wt} \% \mathrm{CH}_{4}-$ injected and produced water

Figure 4.42 shows the injected and produced water during the experiment with steam plus $10.0 \mathrm{wt} \%$ propane. Steam with a cold-water equivalent of $894 \mathrm{~mL}$ was injected during this experiment. Approximately all the injected water was recovered.

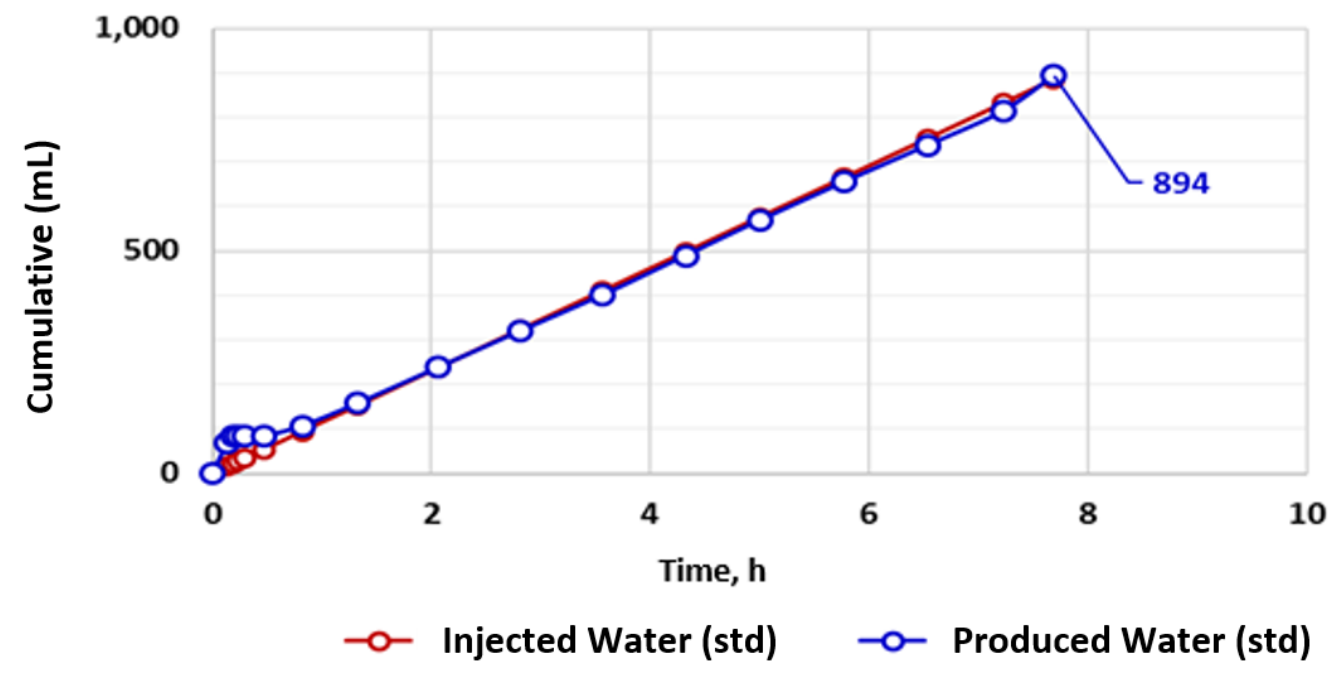

Figure 4.42 Steam plus $10.0 \mathrm{wt} \% \mathrm{CH}_{4}$ - injected and produced water 
An interesting observation from the produced water plots for methane (Figures 4.40 to 4.42 ) and nitrogen (Figures 4.30 to 4.32 ) is that during the first hour of production, the produced water was slightly higher than the injected water. On the other hand, the produced water from carbon dioxide and propane experiments was similar or even less than the injected water. A possible explanation of this difference is that the volume of the injected methane and nitrogen was higher than the injected volumes of carbon dioxide and propane. The increased injected volume enhances the possibility of early breakthrough between the injection and production wells along with the tendency of producing the water that was present in the model prior to the start of injection process.

Figure 4.43 shows the injected and produced gas during the experiment with steam plus $5.0 \mathrm{wt} \%$ methane. A total of 66.0 std litres of gas was injected. Approximately $6 \%$ of the injected gas remained in the model, with 35.9 std litres being produced. The amount of gas retention was similar to that of the $5.0 \mathrm{wt} \%$ nitrogen experiment. Unlike in the experiments with other gases, not much delay was observed in the starting times of oil and gas production.

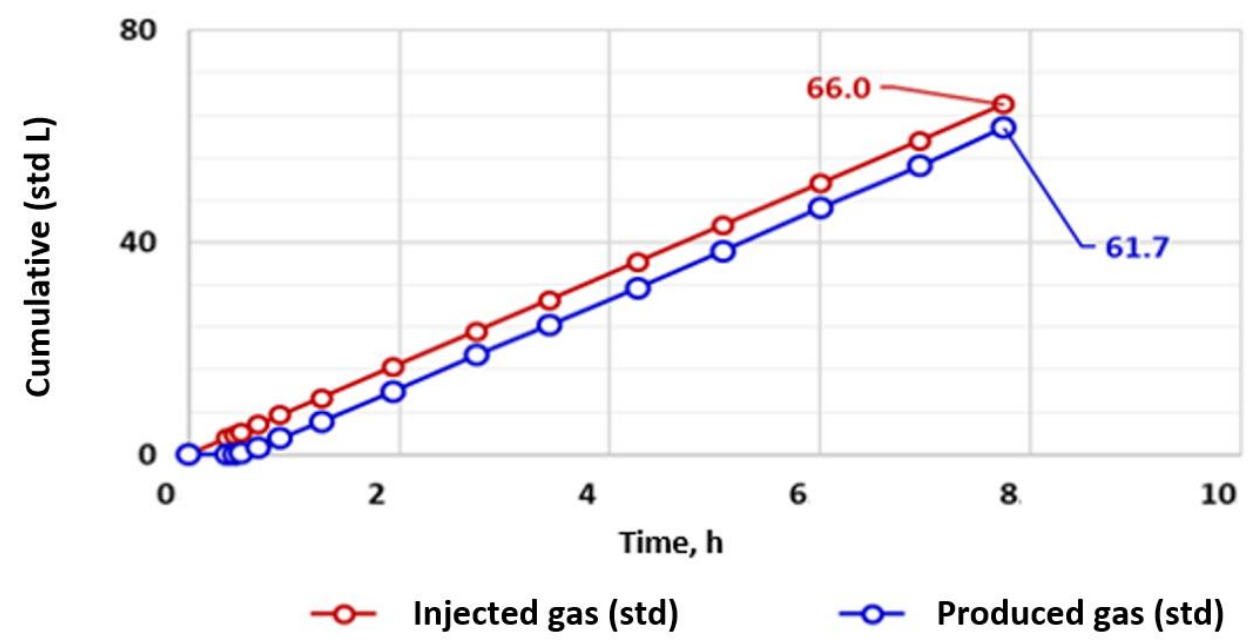

Figure 4.43 Steam plus 5.0 wt $\% \mathrm{CH}_{4}$ - injected and produced gas

Figure 4.44 shows the injected and produced gas during the experiment with steam plus $7.5 \mathrm{wt} \%$ methane. A total of 98.6 std litres of gas was injected, of which 95.8 litres was recovered, or about $3 \%$ less than injected. Similarly to the previous experiment with methane, oil and gas started to produce at the same time. 
Figure 4.45 shows the injected and produced gas during the experiment with steam plus $10.0 \mathrm{wt} \%$ nitrogen. A total of 131.5 std litres of gas was injected, of which 124.4 litres was recovered. This corresponds to approximately 5\% solvent retention. Again, during this experiment, the oil and gas breakthrough times were identical.

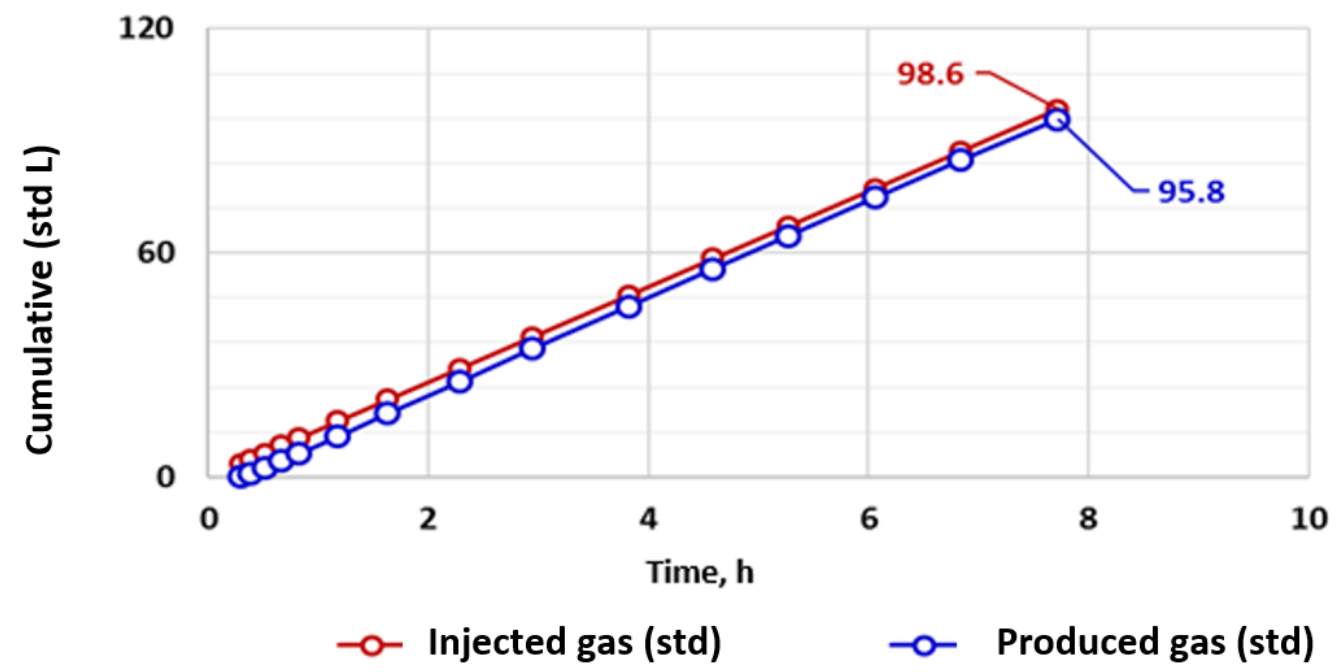

Figure 4.44 Steam plus $7.5 \mathrm{wt} \% \mathrm{CH}_{4}$ - injected and produced gas

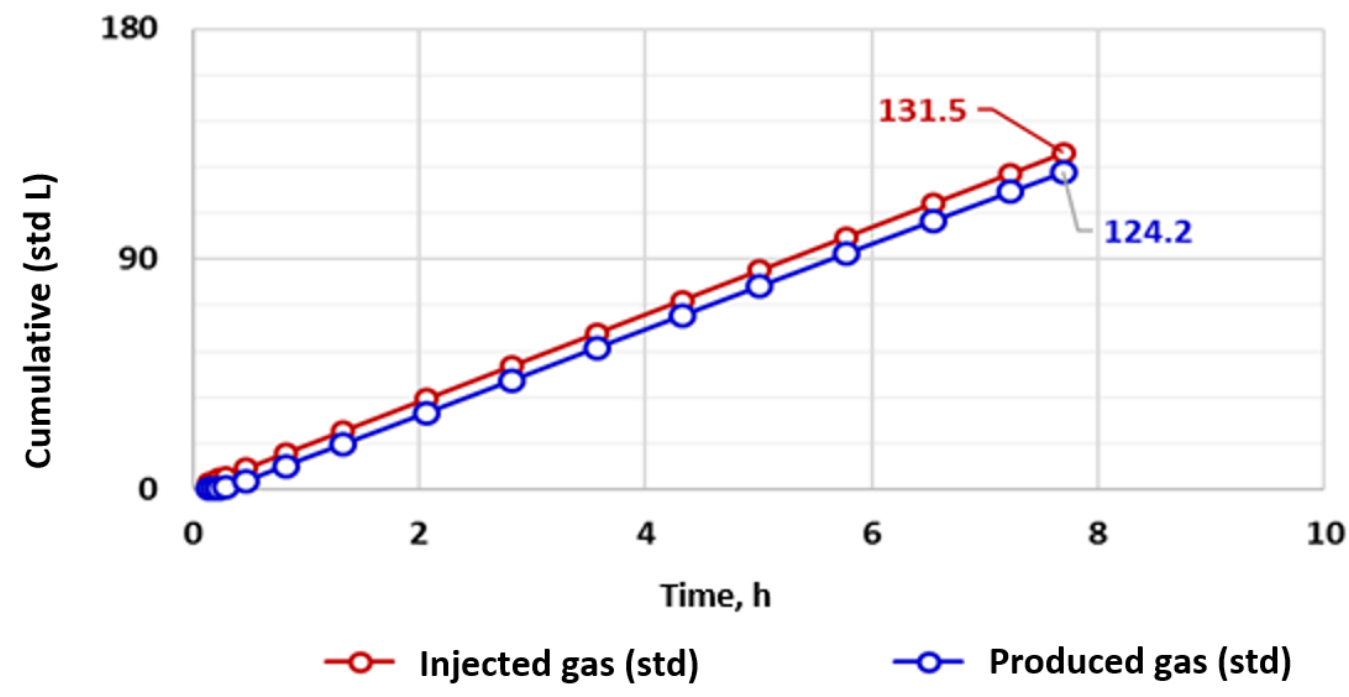

Figure 4.45 Steam plus $10.0 \mathrm{wt} \% \mathrm{CH}_{4}$ - injected and produced gas 


\subsection{Effect of Solvent Type on Hybrid SAGD Performance}

In this section, the experimental results are analyzed to evaluate the most suitable solvent for the hybrid SAGD process.

Figure 4.46 presents the comparison of percentage recovery of original oil in place (\%OOIP) for carbon dioxide, propane, nitrogen and methane, when these solvents are mixed separately with steam at a concentration of $5.0 \mathrm{wt} \%$. Propane seemed to yield the highest \%OOIP recovery among all these solvents. Methane shows the second highest recovery, while carbon dioxide and nitrogen resulted in similar recoveries. Quantitatively, the \%OOIP recoveries from propane, methane, nitrogen and carbon dioxide were 42.6, 38.2, 36.0 and 36.5 percent, respectively.

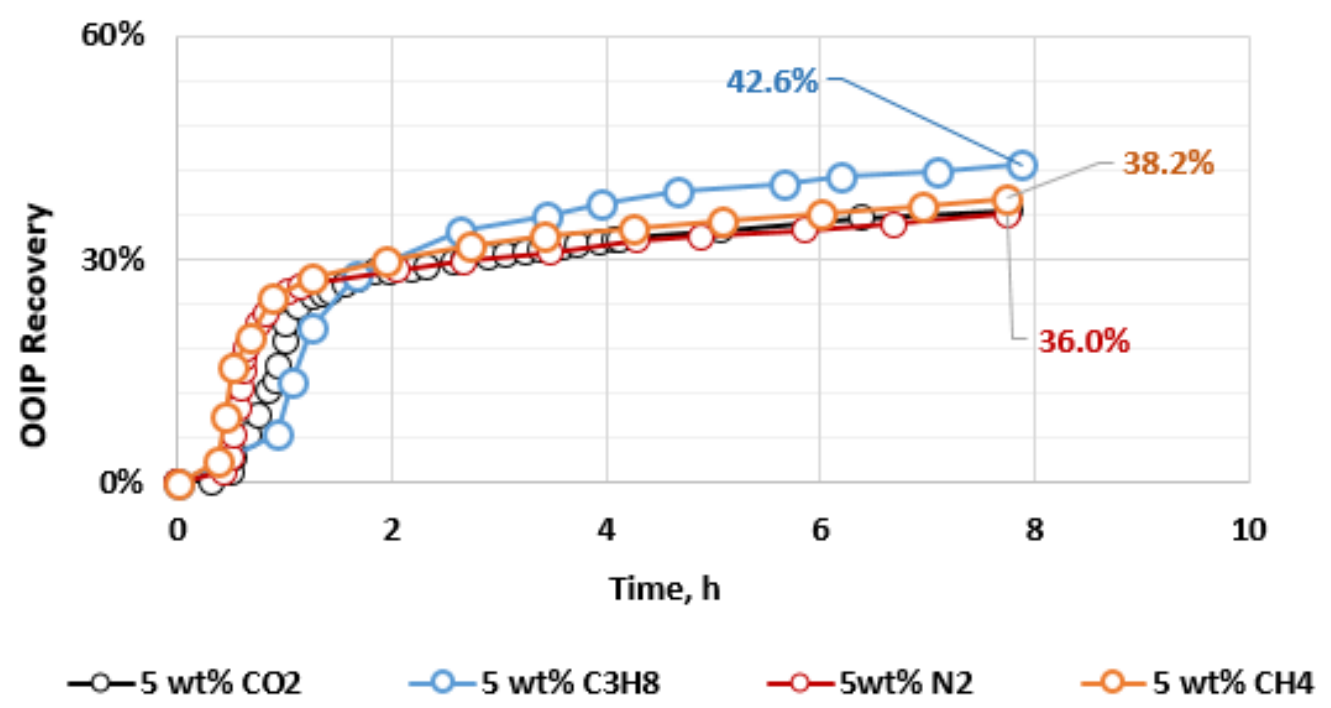

Figure 4.46 5.0 wt\% solvent injection - Solvent effect on \%OOIP recovery

The slopes of the production curves indicate that methane and nitrogen started to produce oil much quicker with steeper slopes compared to carbon dioxide and propane. This shows that nitrogen and methane, being less soluble in oil and exhibiting more expansion at the model temperature, pushed the oil towards the production well faster than did carbon dioxide and propane. These last two gases, being more soluble in oil, lost the initial expansion effect. 
Another observation from Figure 4.46 is that once the oil production rate started to stall, propane produced oil at a higher rate compared to the other non-condensable gases. This indicates that this improved oil recovery came from further reduction in the oil viscosity, since solubility in oil at model conditions is highest for propane among all the gases tested.

Figure 4.47 compares \%OOIP recovery for carbon dioxide, propane, nitrogen and methane, when these solvents are mixed separately with steam at a concentration of $7.5 \mathrm{wt} \%$. Unlike the results for $5.0 \mathrm{wt} \%$ addition, this time methane showed the highest oil recovery. Quantitatively, the \%OOIP recoveries from methane, propane, nitrogen and carbon dioxide were 37.4, 33.7, 32.0 and 31.0 percent, respectively.

The slopes of the production curves indicate that, as in the $5.0 \mathrm{wt} \%$ experiments, methane and nitrogen started to produce oil quicker with a steeper slope compared to carbon dioxide and propane. Also, propane's contribution to the oil production came at a substantial delay compared to the NCGs.

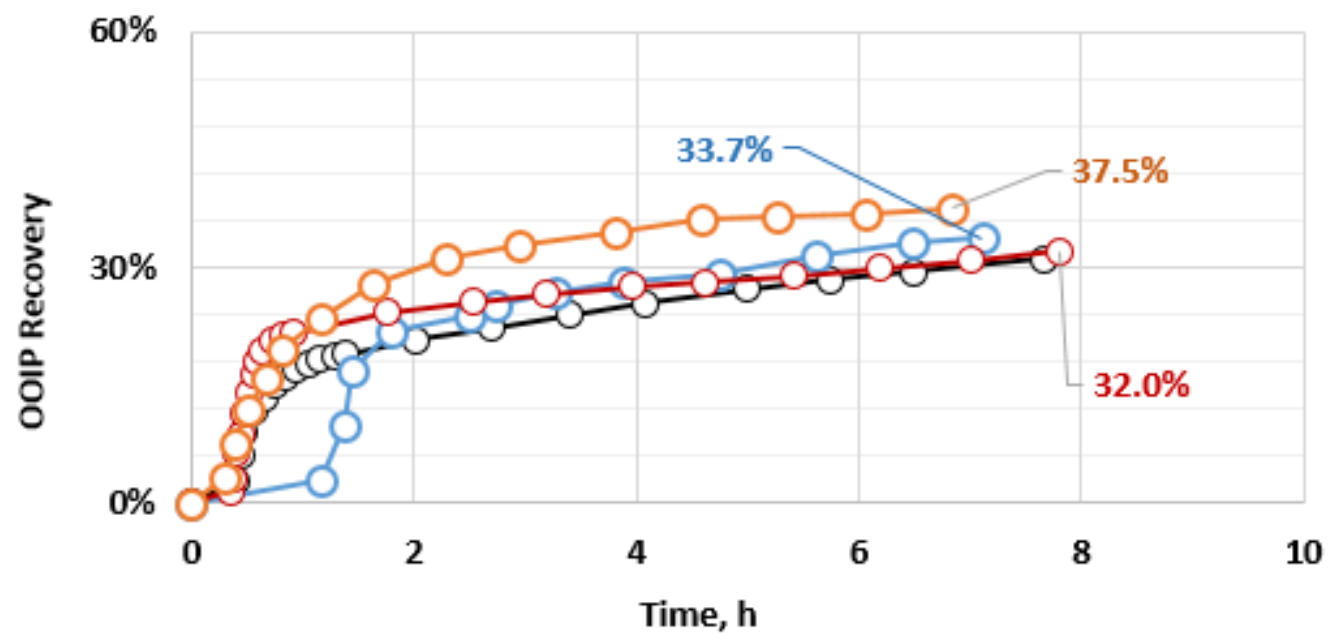

$-0-7.5$ wt\% CO2 $-0-7.5$ wt\% C3H8 $-0-7.5 w t \%$ N2 $\quad-0-7.5$ wt\% CH4

Figure 4.47 7.5 wt\% solvent injection - solvent effect on \%OOIP recovery

Figure 4.48 compares the \%OOIP recovery for carbon dioxide, propane, nitrogen and methane, when these solvents are mixed with steam at a concentration of $10.0 \mathrm{wt} \%$. This time methane 
showed the highest oil recovery, with nitrogen having the second highest. Quantitatively, the $\%$ OOIP recoveries from methane, nitrogen, propane and carbon dioxide were 50.8, 44.3, 34.6 and 29.3 percent, respectively.

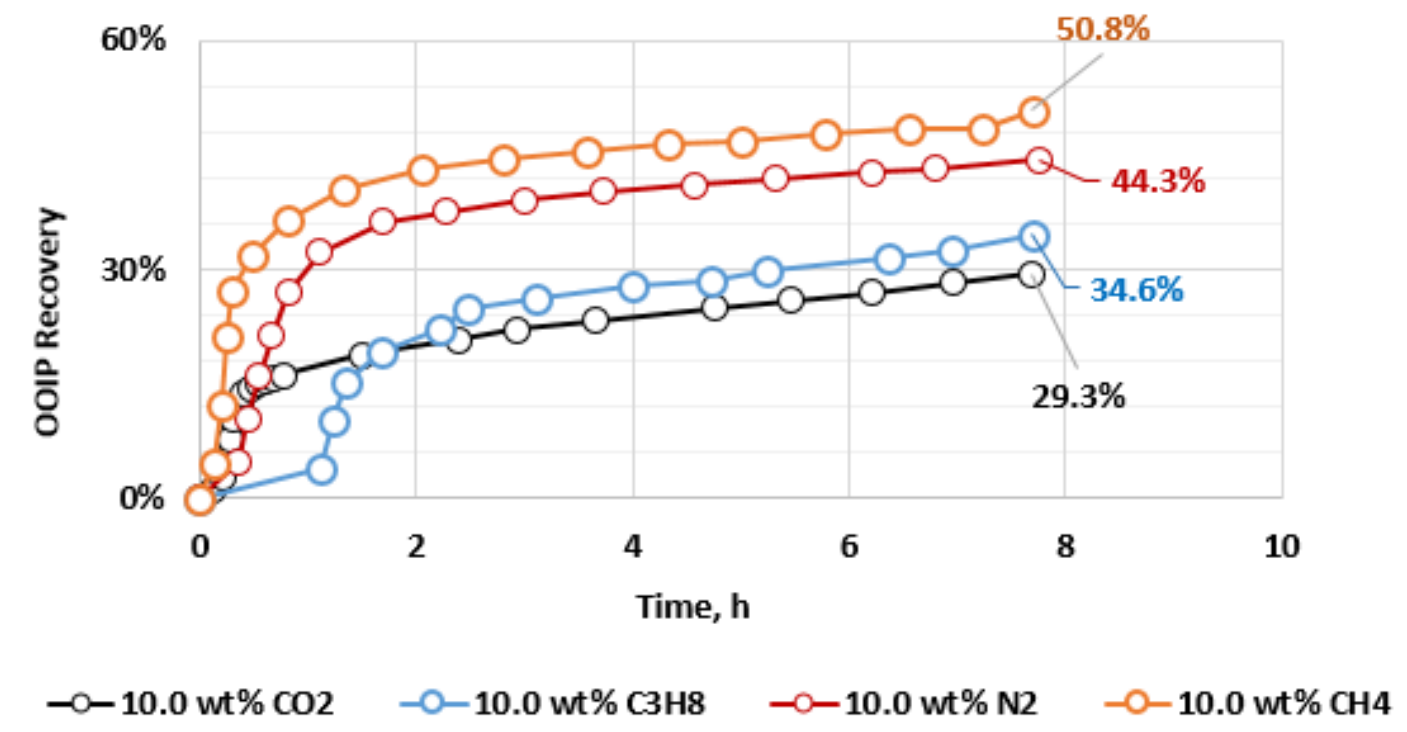

Figure 4.48 10.0 wt\% solvent injection - solvent effect on \%OOIP recovery

The slope of the production curves indicate that methane and nitrogen started to produce oil quicker with a steeper slope compared to carbon dioxide and propane. Also, propane contributed towards oil production with a substantial delay compared to NCGs. This behaviour was found consistently in all other experiments. Furthermore, carbon dioxide seemed to produce the least amount of oil among all the solvents tested.

One observation from these experiments is that-for the highest concentration in the injected stream - the gases that are less soluble in oil, like methane and nitrogen, exhibited the highest oil recovery. In contrast, the gases that are more soluble in oil, like carbon dioxide and propane, showed the lowest oil recovery for the highest concentration in the injected stream. Furthermore, less soluble gases like nitrogen and methane showed potential for higher oil recovery compared to more soluble gases like carbon dioxide and propane. 
At run conditions, nitrogen is about three times less soluble in oil than is carbon dioxide; thus, the oil viscosity with maximum dissolved nitrogen concentration will be about $8 \mathrm{cP}$, calculated using the viscosity correlation by Freitag (2015). Similarly, methane solubility is about $30 \%$ less than carbon dioxide solubility, and the oil viscosity with maximum dissolved methane concentration will be about $6 \mathrm{cP}$, calculated using the viscosity correlation by Freitag (2015). The diluted oil viscosities for both nitrogen and methane were higher than for carbon dioxide and propane, even though these first two gases produced more oil. This indicates that additional viscosity reduction probably does not play much role in enhancing the hybrid SAGD recovery performance.

One significant difference among the gases tested is that methane and nitrogen have lower molecular weights compared to carbon dioxide and propane. More prominently, both of the less soluble gases have very low critical temperatures compared to the more soluble gases, and their saturation pressure lies in below-zero regions (Figure 4.49). It is likely that gases with their saturation pressure in below-zero regions are more suitable for the hybrid SAGD process.

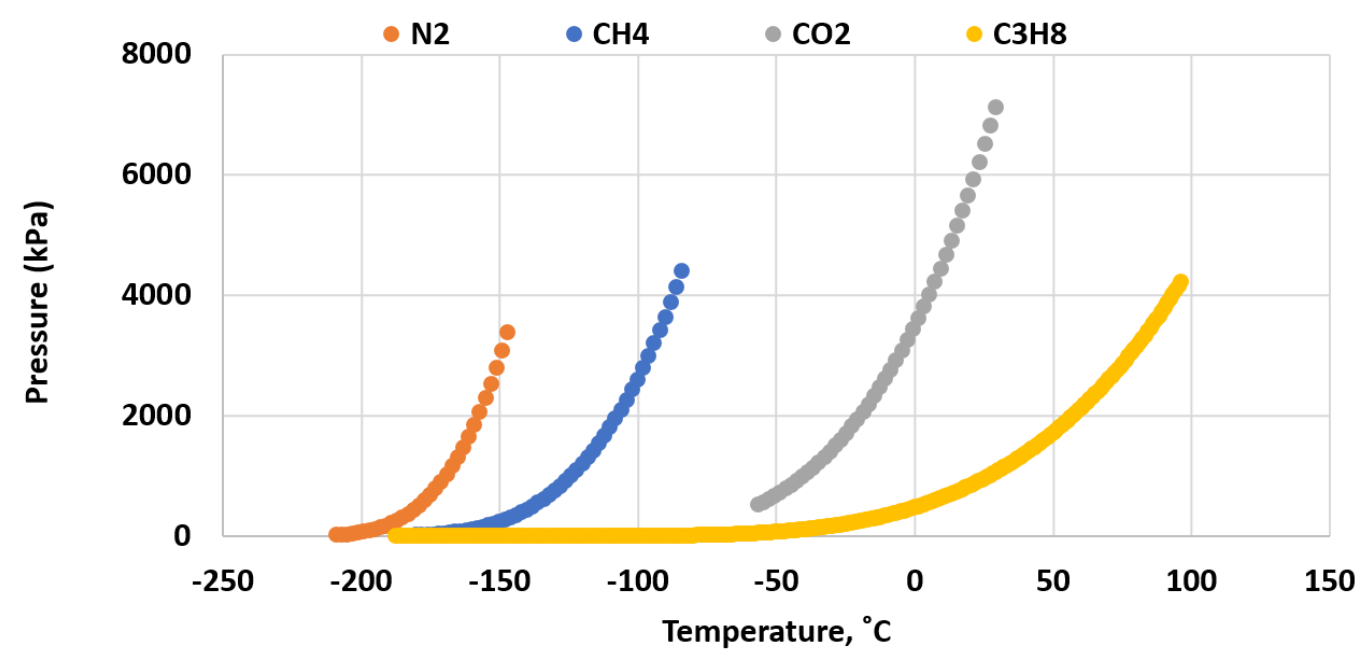

Figure 4.49 Solvent saturation properties (http://webbook.nist.gov/chemistry/fluid/)

One explanation of increased oil recovery from less soluble gases is that, on a mole percent basis, a higher number of moles were being injected for methane and nitrogen. Figure 4.50 presents the solvent mole fraction in the injected stream as a function of \%OOIP recovery. With the increase in solvent mole fraction, the \%OOIP recovery increases but, as mentioned earlier, this holds true 
only for methane and nitrogen. Overall, methane resulted in the highest oil recovery among all these solvents while it was being injected at the highest mole percentage.

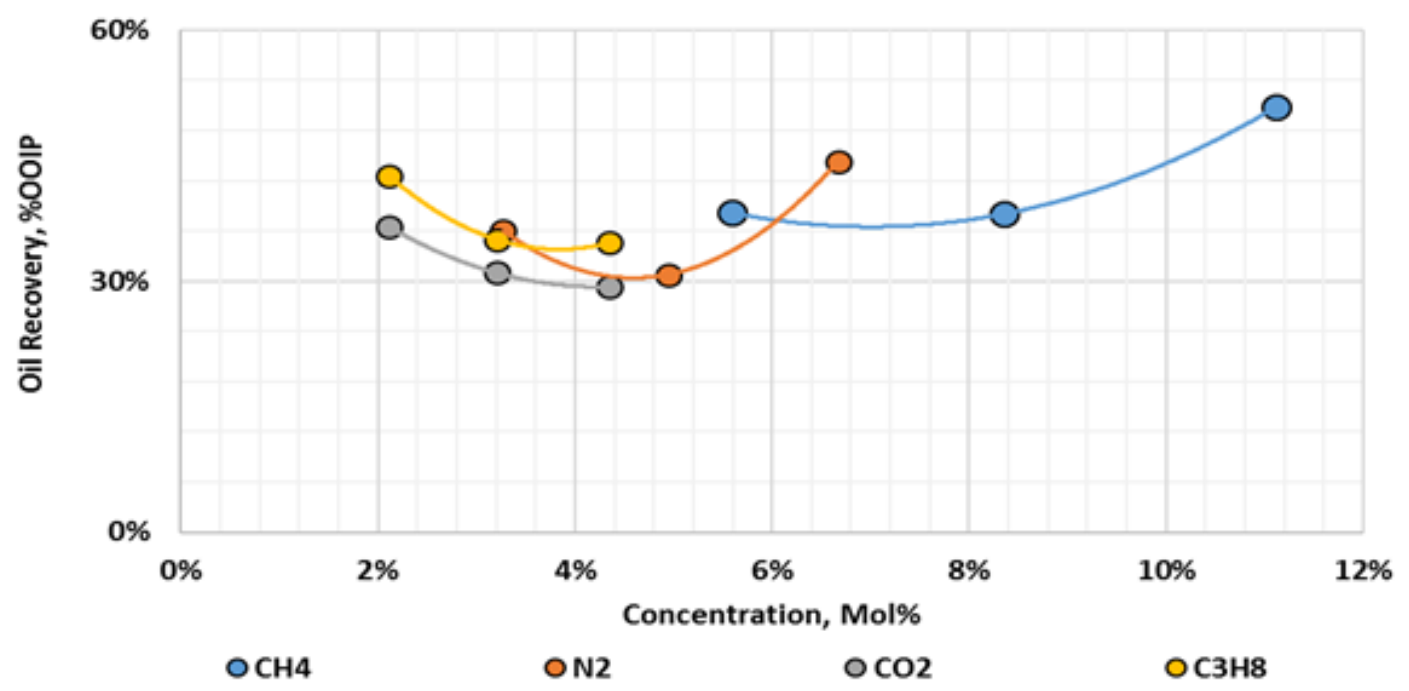

Figure 4.50 Solvent mol\% in injected stream versus oil recovery \%OOIP

The figure also suggests that, at the same mole percent, propane seems to produce more oil than does carbon dioxide; however, the oil recovery from nitrogen was not less than propane. It appears that the lower molecular weight and smaller molecule size solvents (such as methane and nitrogen) has more pronounced effect on increasing the oil recovery. when their concentration in the injected steam is increased. Furthermore, nitrogen that has less solubility compared to propane and carbon dioxide tend to produce larger amount of oil as compared to carbon dioxide and propane. This is possibly because the nitrogen that is lower in molecular weight as compared to propane and carbon dioxide, tend to rise quicker in the model or reservoir and, in addition to providing an insulation effect and reducing heat losses, it displaces the oil from the upper part of the reservoir.

Another observation from these experiments is that methane and nitrogen started to produce oil much quicker, with steeper slopes, compared to carbon dioxide and propane. This shows that nitrogen and methane, being less soluble in oil and lighter than carbon dioxide and propane, quickly rise to the top of the model, surround the matrix and push the oil towards the production well faster than do carbon dioxide and propane. 
Figure 4.51 presents the comparison of solvent retention as a percent of solvent injected for the $5.0 \mathrm{wt} \%$ solvent addition experiments. Solvent retention is the amount of solvent left in the reservoir that is not recovered during the injection/production stage. It is an important parameter for evaluating the economic feasibility of the hybrid SAGD process. Higher solvent retention corresponds to an economically unfavorable process.

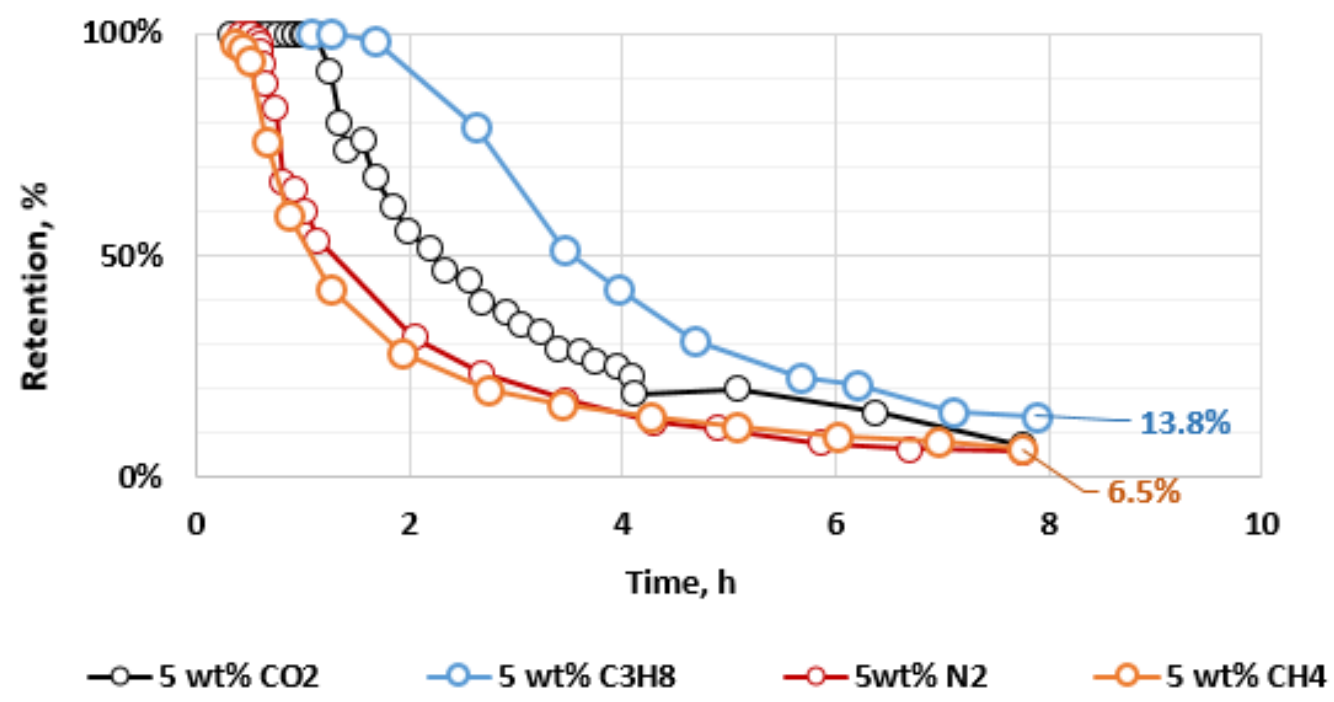

Figure 4.51 5.0 wt\% solvent injection - solvent effect on \%retention

As can be seen from Figure 4.51, during the initial part of the run, when the model was being pressurized, all the injected solvent was held in the model. This was due to effective model pressurization. When the production started, the injected solvent started to be recovered along with the oil. By the end of the production period, the solvent recovery was at its maximum. For the 5.0 $\mathrm{wt} \%$ solvent addition experiments, propane retention was highest. Quantitatively, the solvent retention results for the propane, carbon dioxide, methane, and nitrogen experiments were 13, 7.1, 6.5 , and 5.8 percent of the injected solvent.

Figure 4.52 presents the same comparison for the $7.5 \mathrm{wt} \%$ solvent addition experiments. Again, propane resulted in the highest solvent retention among all the solvent gases tested. Quantitatively, the solvent retention results for propane, nitrogen, methane, and carbon dioxide experiments were $15,6.8,2.9$, and 0.2 percent of the injected solvent. 


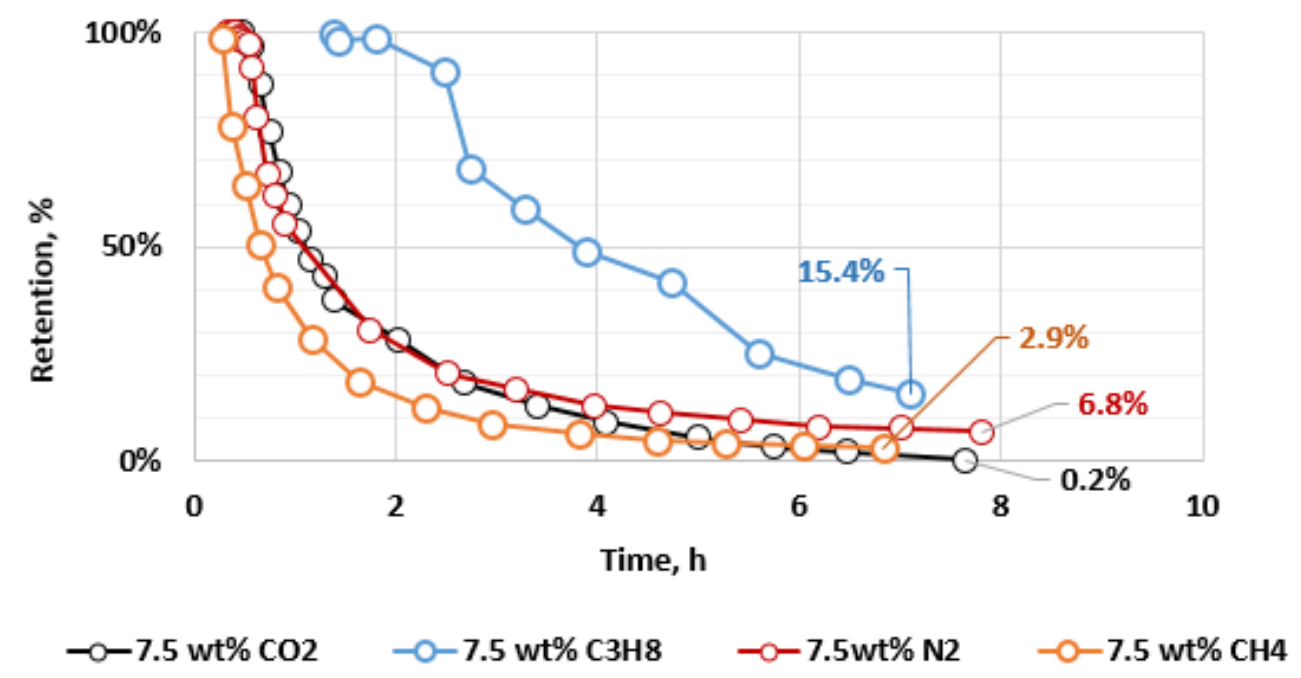

Figure 4.52 7.5 wt\% solvent injection - solvent effect on \%retention

Figure 4.53 presents the same comparison for the $10.0 \mathrm{wt} \%$ solvent addition experiments. Propane resulted in the highest solvent retention among all the solvent gases tested. Quantitatively, the solvent retention results for the propane, methane, nitrogen, and carbon dioxide experiments were $16,5.6,5.3$, and 1.0 percent of the injected solvent.

At any concentration, propane retention in the model was highest among all the solvent gases tested, due to its highest solubility in the oil phase; as well, since not all the diluted oil was produced, the solvent dissolved in it increased the amount of retention.

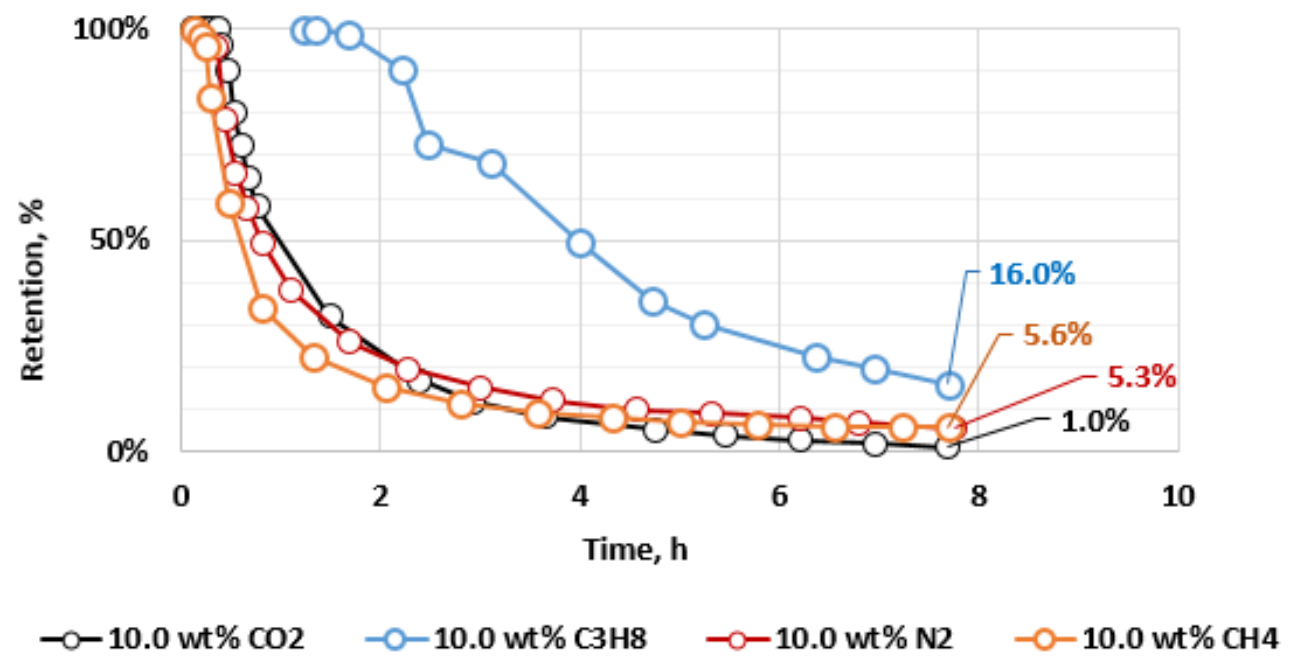

Figure 4.53 10.0 wt \% solvent injection - solvent effect on \%retention recovery 


\subsection{Summary of Important Observations and Findings}

The important observations and findings from the experimental study are summarized below:

$\checkmark$ The baseline test with steam only resulted in the lowest recovery of $10.6 \%$ of the original oil in place (OOIP). This recovery is much lower than that for a SAGD type of process, where up to $60 \%$ recovery can be achieved during the rising and spreading phase of the process. The reason for such a low recovery from the laboratory scale model was the low temperature and pressure conditions of the experiment.

$\checkmark$ Among the three concentrations of carbon dioxide tested, the lowest concentration of 5.0 wt $\%$ showed the highest oil recovery of 36.5\% OOIP, whereas the highest concentration of $10.0 \mathrm{wt} \%$ had the lowest recovery of $29.3 \%$ OOIP. The recovery from the intermediate concentration of $7.5 \mathrm{wt} \%$ was $30.9 \%$, slightly higher than from the $10.0 \mathrm{wt} \%$ run.

$\checkmark$ Among the three concentrations of propane tested, the lowest concentration of $5.0 \mathrm{wt} \%$ showed the highest oil recovery of $42.6 \%$. OOIP, whereas the highest concentration of 10.0 $\mathrm{wt} \%$ had the lowest recovery of $34.6 \%$ OOIP. The recovery from the $7.5 \mathrm{wt} \%$ run was $35.0 \%$ OOIP, slightly higher than from the $10.0 \mathrm{wt} \%$ experiment.

$\checkmark$ Among the three concentrations of nitrogen tested, the highest concentration of $10.0 \mathrm{wt} \%$ showed the highest oil recovery of $44.3 \%$ OOIP, whereas the intermediate concentration of $7.5 \mathrm{wt} \%$ showed the lowest recovery of $30.8 \%$ OOIP. The recovery from the lowest concentration of $5.0 \mathrm{wt} \%$ was $36.0 \%$ OOIP.

$\checkmark$ Among the three concentrations of methane tested, the highest concentration of $10.0 \mathrm{wt} \%$ showed the highest oil recovery of 50.8\% OOIP, whereas the $7.5 \mathrm{wt} \%$ and $5.0 \mathrm{wt} \%$ showed similar recoveries of about $38 \%$.

$\checkmark$ For low-pressure reservoirs, that are not too shallow to mine and too deep to apply conventional SAGD, hybrid SAGD performed much better than conventional SAGD. The 
oil recoveries from the addition of solvents were at least 18 percentage points higher than that from the steam only experiment.

$\checkmark$ The lower-molecular-weight, readily available, and less soluble solvents like methane and nitrogen performed better than the higher-molecular-weight, and more soluble solvents like propane and carbon dioxide.

$\checkmark$ Methane and nitrogen started to produce oil much quicker, with steeper slopes, compared to carbon dioxide and propane. Methane and nitrogen showed a tendency to rise quickly to the top of the model, surrounding the matrix and pushing the oil towards the production well faster than did carbon dioxide and propane. Further experiments are required to validate this hypothesis. Experiments can be designed using a computed tomography (CT) scanner in which gas movement inside the matrix can be monitored.

$\checkmark$ On an equal weight percentage basis, addition of methane resulted in the highest oil recovery of $50.7 \%$ OOIP.

$\checkmark$ The solvent retention for propane was highest, up to $15 \%$ of the amount injected, among all the solvents tested.

$\checkmark$ For low pressure reservoirs, hybrid SAGD with methane or nitrogen addition seems a viable process. 


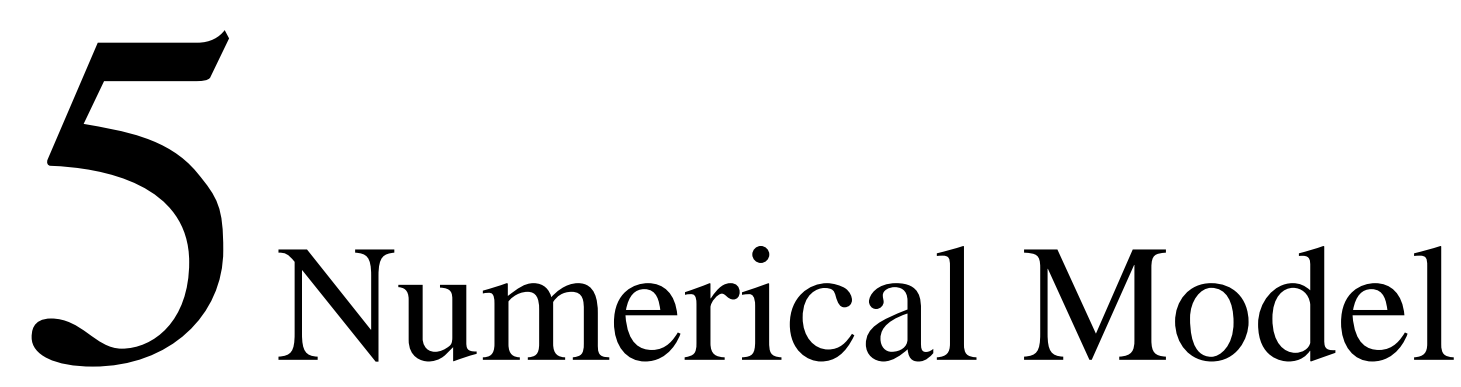

\section{Setup}

In reservoir engineering, numerical modelling/simulation is a widely applied technique and is a powerful tool used for understanding the fluid flow dynamics in the reservoir. It uses mathematical models to describe the physical and chemical phenomena and simulate an enhanced oil recovery process. The mathematical models are derived in the form of equations based on laws of conservation of mass, energy and momentum. Some of these equations, such as partial differential equations, are difficult to solve directly and require numerical techniques such as finite difference, finite element or finite volume.

This chapter presents the methodology to develop the numerical model used to history-match the experimental data for produced oil, water and gas. Commercial software packages by Computer Modelling Group (CMG) were used to perform the simulations. CMG's WinProp software was used to generate the fluid model, and CMG's STARS software was used to generate the reservoir model and perform the history-match. The fundamental equations, along with important assumptions and initial and boundary conditions, are presented in this chapter.

\subsection{Equation of State (EOS) Fluid Model}

In numerical modelling, it is very important to capture the true phase behaviour of the solvent-oil system of interest. To accurately model a hybrid thermal process, the numerical model requires 
the viscosity and density as a function of temperature and dissolved solvent concentration. The maximum solubility of the solvent at a given temperature and pressure condition is also required.

CMG's WinProp is a compositional equation of state (EOS) based phase behaviour and property calculation package. It features techniques for characterizing the heavy oil or bitumen, matching laboratory pressure/volume/temperature (PVT) data through regression, phase diagram generation and process flow simulations (CMG Winprop User Guide). WinProp allows calculation of saturation pressure and temperature, two-phase and multiple-phase flash, compositional gradient, solubility of light gases in water or brine, critical properties via correlations, etc. The fundamental equations used in WinProp are presented in Appendix B.

To solve the EOS, the critical temperature, critical pressure, acentric factor and interaction coefficient are required. This information is already provided in WinProp for some common petroleum mixtures. For fluids like volatile oils, gas condensate, heavy oil or their mixture with solvents like carbon dioxide, methane, propane, nitrogen, etc., the component parameters in the EOS are adjusted or tuned via regression to match the experimental data. Examples of parameters to be matched are dead oil density and viscosity, diluted oil density and viscosity, equilibrium kvalues (i.e., solvent solubility in the oil phase), and saturation pressures. The available parameters for regression to match density are volume shift parameters; to match viscosities are viscosity correlation coefficients; to match saturation pressures are hydrocarbon interaction coefficients; and to modify all properties are critical temperature, critical pressure, acentric factor and interaction coefficient.

The objective function of the regression involves the solution of complex nonlinear equations including flash and saturation-pressure calculations. WinProp uses a modified adaptive leastsquares algorithm of Dennis et al (1983) that involves the use of nonlinear optimization concepts on direction and step-size selection.

In this study, to generate a tuned fluid model and to simulate the experimental data, first the dead oil density and viscosity were matched by providing the molecular weight, API gravity, critical temperature and critical pressure information. The Peng-Robinson (1976) EOS was used, and 
appropriate regression parameters were selected for the history-match. The modified Pedersen and Fredenslund (1987) model was selected for regressing the viscosity data.

Figure 5.1 presents the history-match of the dead oil density at three temperatures of 15,25 and $40^{\circ} \mathrm{C}$ and at atmospheric pressure. The blue markers and line correspond to the experimental data and the orange markers and line correspond to the simulated data. The relative error with respect to experimental data is presented along the y-axis. For the highest temperature of $40^{\circ} \mathrm{C}$, the error was lowest, $0.13 \%$. For the lowest temperature of $25^{\circ} \mathrm{C}$, the error was highest, $0.74 \%$.

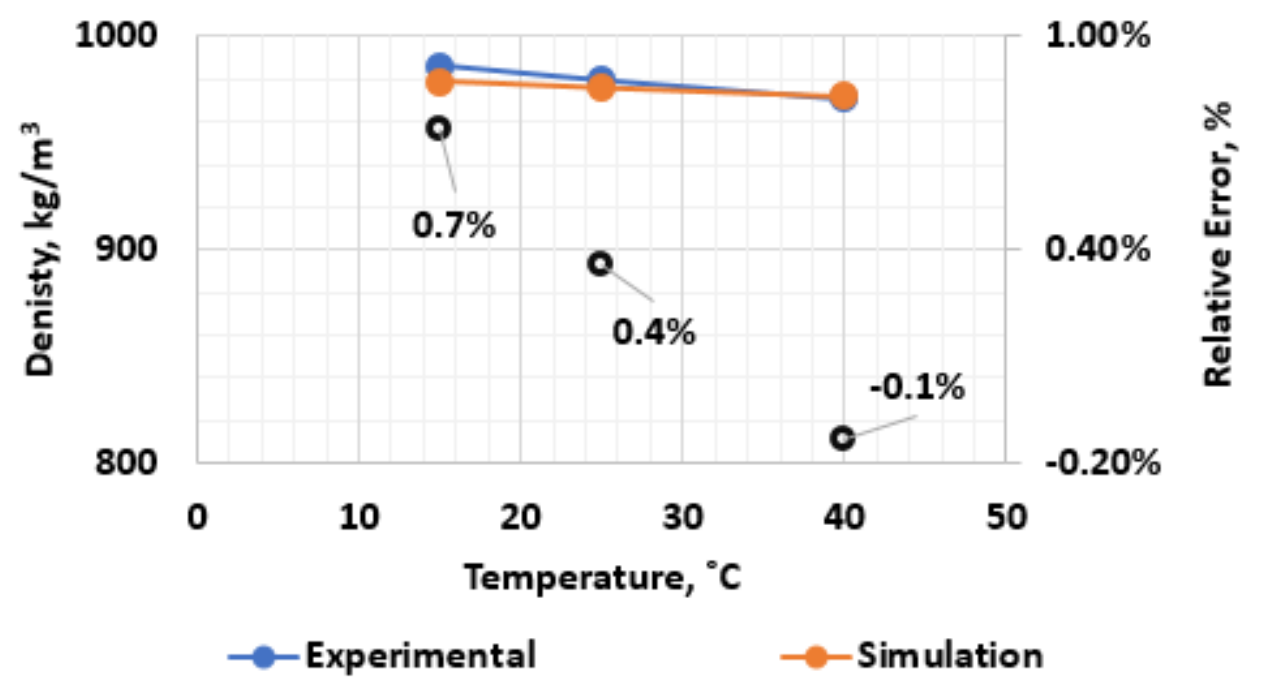

Figure 5.1 History-matched dead oil density comparison at atmospheric pressure and different temperatures

Figure 5.2 presents the comparison of experimental with the simulated data for dead oil density at different pressures of $500,1500,2500$ and $3500 \mathrm{kPa}$ and temperature of $21^{\circ} \mathrm{C}$. For the highest pressure of $3500 \mathrm{kPa}$, the error was highest, $0.58 \%$. For the lowest pressure of $500 \mathrm{kPa}$, the error was lowest, $0.48 \%$.

Figure 5.3 presents the comparison of experimental with the simulated data for dead oil density at different pressures of 500, 1500, 2500, 3500, 5000 and 10,000 $\mathrm{kPa}$ and temperature of $35^{\circ} \mathrm{C}$. For the highest pressure of $10,000 \mathrm{kPa}$, the error was lowest, $0.05 \%$. For the lowest pressure of 500 $\mathrm{kPa}$, the error was highest, $0.23 \%$. 


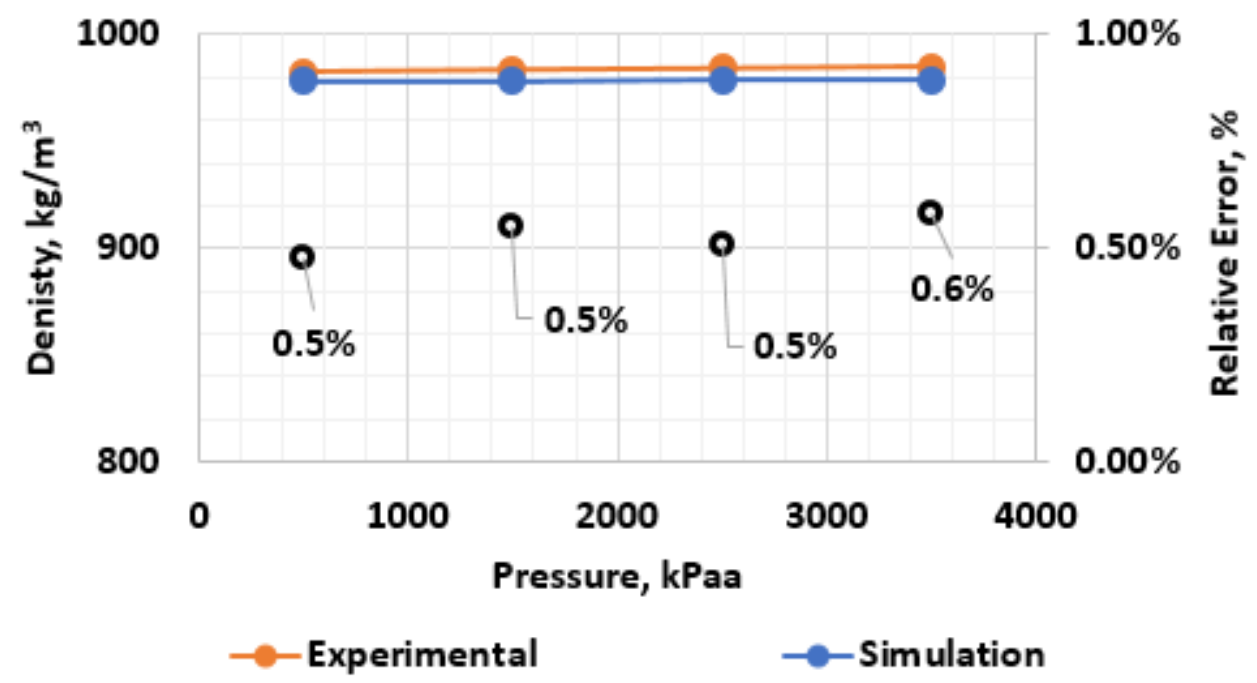

Figure 5.2 History-matched dead oil density comparison at $21^{\circ} \mathrm{C}$ and different pressures

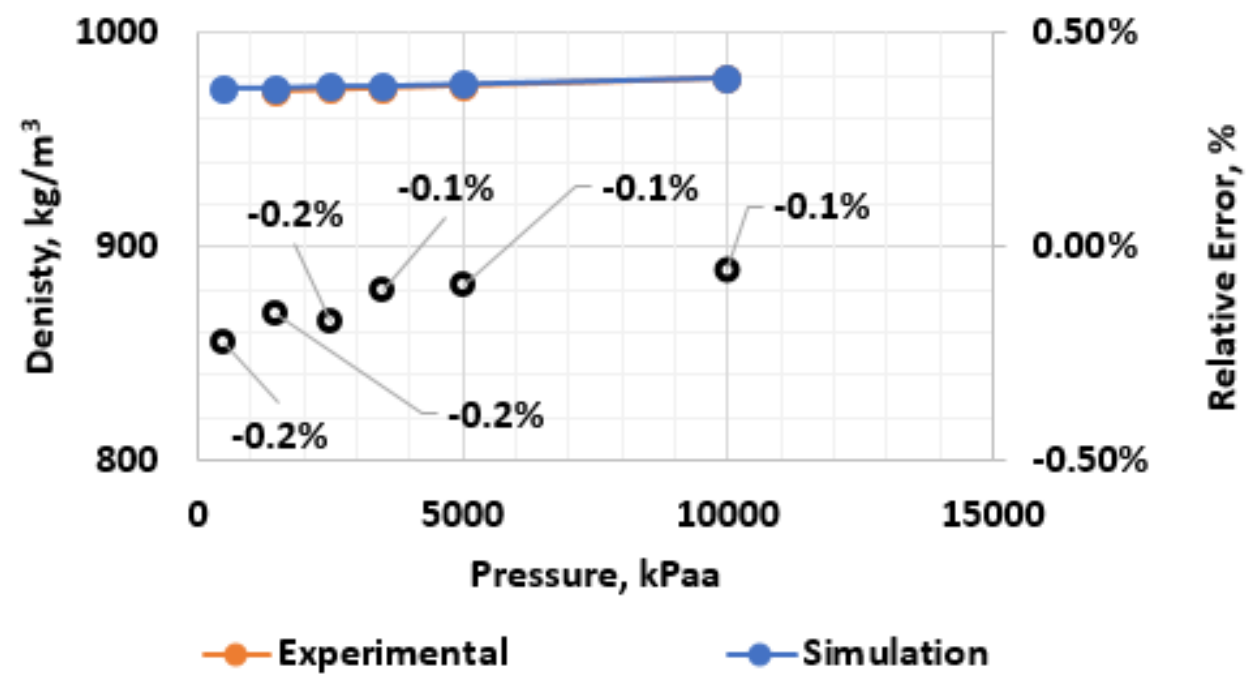

Figure 5.3 History-matched dead oil density comparison at $35^{\circ} \mathrm{C}$ and different pressures

Figure 5.4 presents the comparison of experimental with the simulated data for dead oil viscosity at different temperatures of 15,25 and $40^{\circ} \mathrm{C}$ and at atmospheric pressure. For the lowest temperature of $15^{\circ} \mathrm{C}$, the error was highest, $26.5 \%$. For the highest temperature of $40^{\circ} \mathrm{C}$, the error was lowest, $13.1 \%$. 


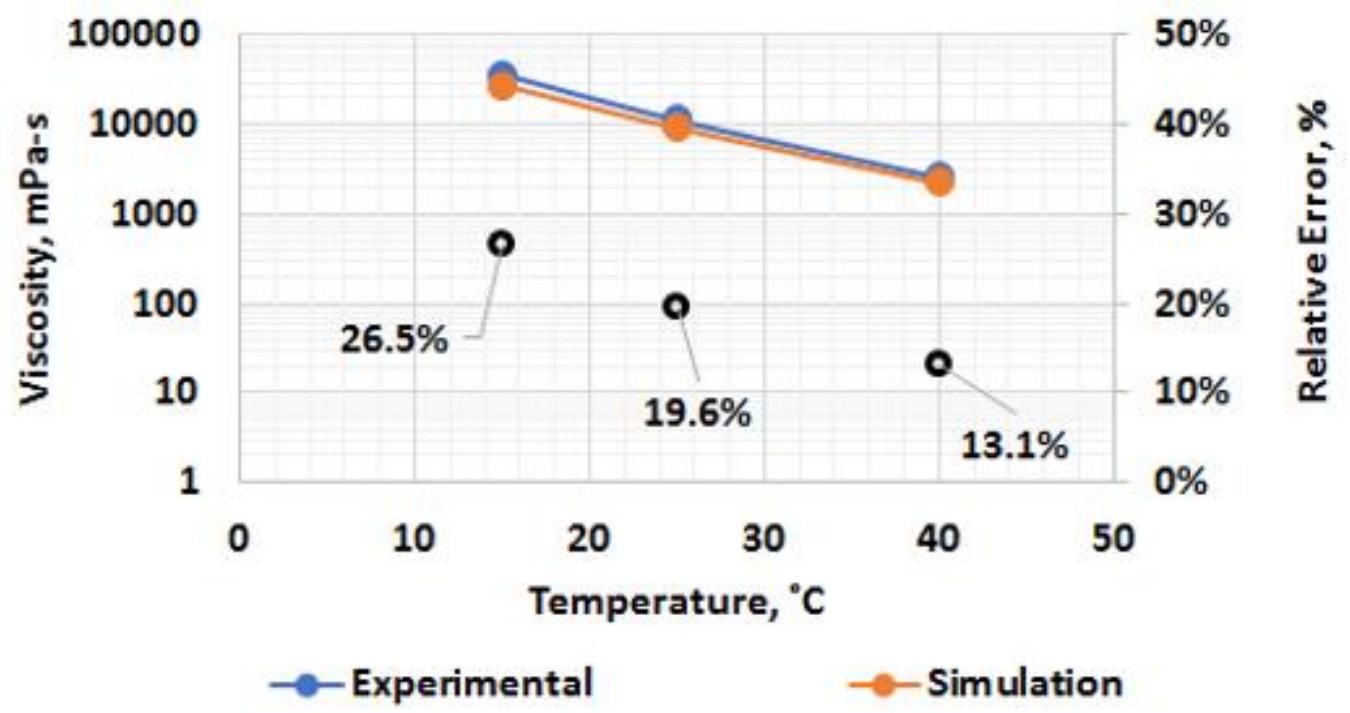

Figure 5.4 History-matched dead oil viscosity comparison at atmospheric pressure and different temperatures

Figures 5.5 presents the comparison of experimental with the simulated data for dead oil viscosity at different pressures of 500, 1500, 2500 and $3500 \mathrm{kPa}$ and temperature of $21^{\circ} \mathrm{C}$. The lowest error of $15.7 \%$ was for the $1500 \mathrm{kPa}$ measurement, and the highest error of $18.4 \%$ was for the $500 \mathrm{kPa}$ measurement.

Figures 5.6 presents the comparison of experimental with the simulated data for dead oil viscosity at different pressures of $500,1500,2500,3500,5000$ and $10,000 \mathrm{kPa}$ and temperature of $35^{\circ} \mathrm{C}$. The lowest error of $0.55 \%$ was for the measurement at the highest pressure of $10000 \mathrm{kPa}$; and the highest error of $13.5 \%$ was for that at the lowest pressure of $500 \mathrm{kPa}$.

Compared to viscosity, the density of the dead oil was matched more closely. Especially at lower temperatures, the viscosity match was rather poor; the relative error was above $10 \%$. However, with many data points being matched, overall a reasonable history-match was obtained for the dead oil density and viscosity. 


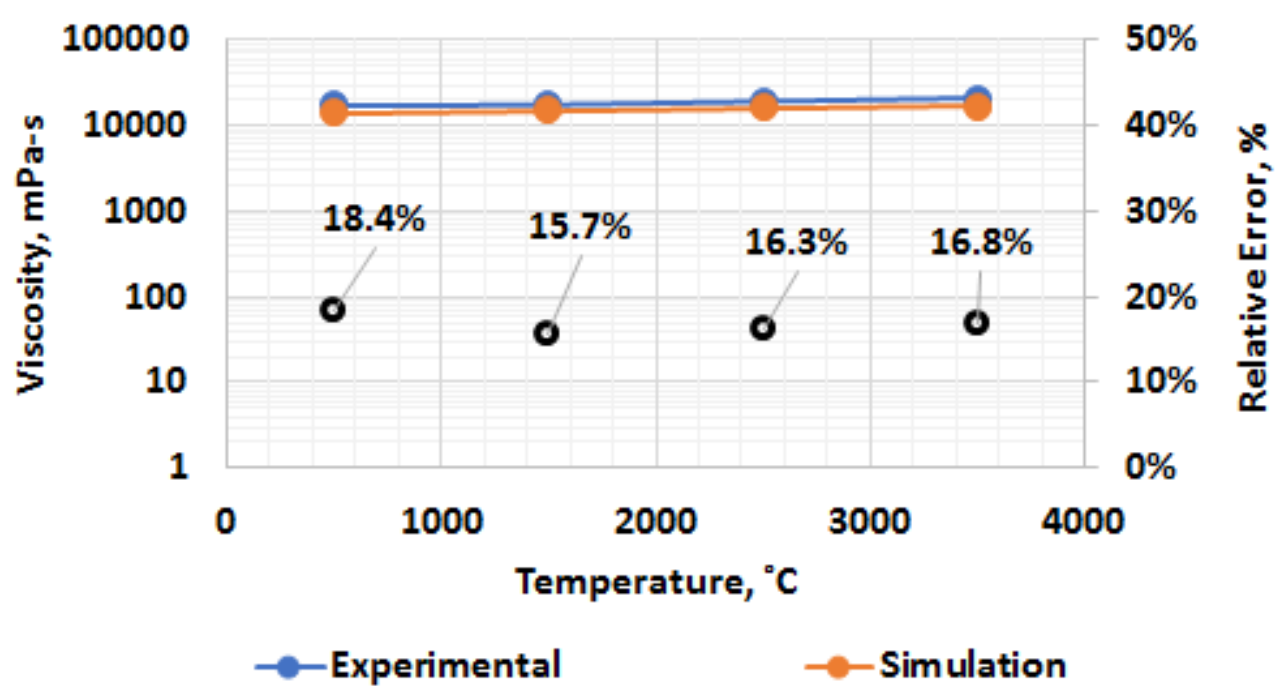

Figure 5.5 History-matched dead oil viscosity comparison at $21^{\circ} \mathrm{C}$ and different pressures

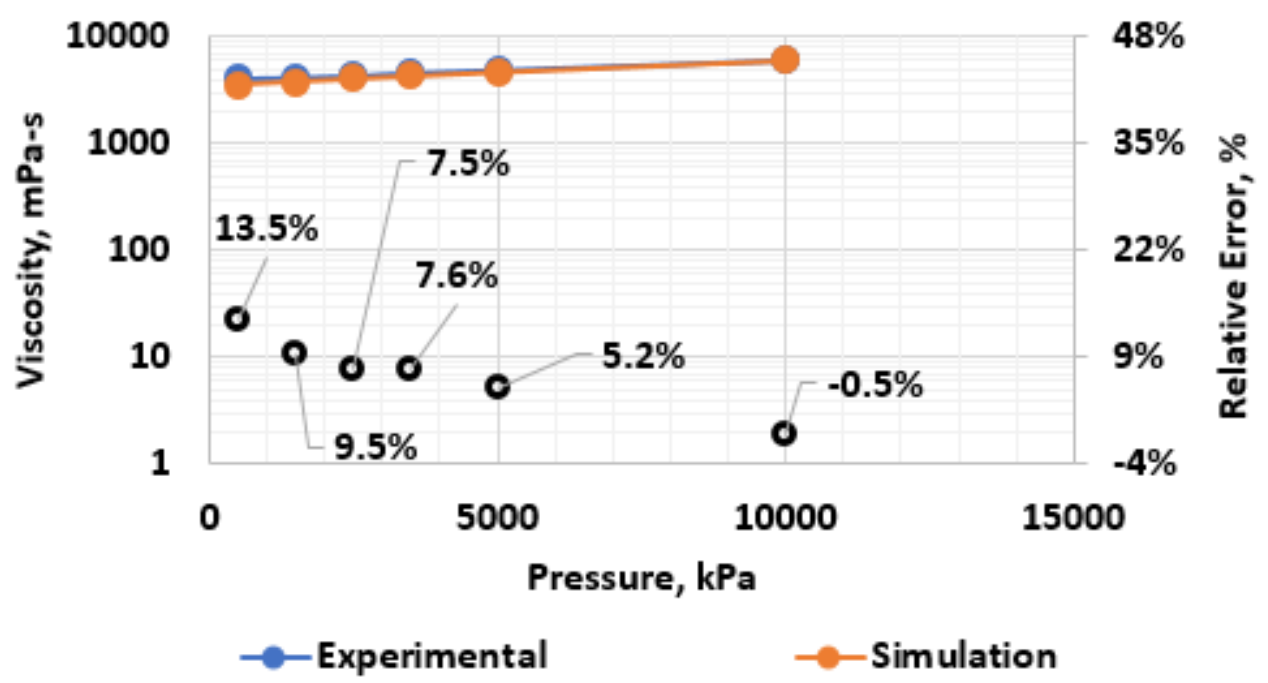

Figure 5.6 History-matched dead oil viscosity comparison at $35^{\circ} \mathrm{C}$ and different pressures

Once the fluid model was tuned for dead oil, it was further tuned for the solubility of the solvents like carbon dioxide, methane and propane in the oil phase. The experimental data (Freitag, 2008) of equilibrium k-values and corresponding density and viscosity of the diluted oil at different temperature and pressure conditions are provided in Appendix C. These data were used to further tune the fluid model for solvent-oil systems using WinProp regression techniques. 
Once a tuned model was achieved, the equilibrium k-value tables and viscosity tables were generated for the STARS simulator. These data are presented in Tables 5.1 to 5.4 for carbon dioxide, propane, methane and nitrogen, respectively. In order to cover the range of temperatures and pressures presented in these tables, the data were extrapolated. Note that the experimental PVT data was taken from another study conducted at Saskatchewan Research Council (Freitag, 2008). The data for the nitrogen-oil system was generated by using the data generated by Mehrotra and Svrcek (1982). From the published data the solubility (at a given temperature and pressure condition) of nitrogen and carbon dioxide was evaluated and a ratio of the nitrogen to carbon dioxide solubility was calculated. The calculated solubility ratio was used to generate the k-value table for nitrogen. Figure 5.7 shows the solubility (in $\mathrm{cm}^{3} / \mathrm{cm}^{3}$ ) of nitrogen and carbon dioxide measured by Mehrotra and Svrcek (1982) at different temperature and pressure conditions.

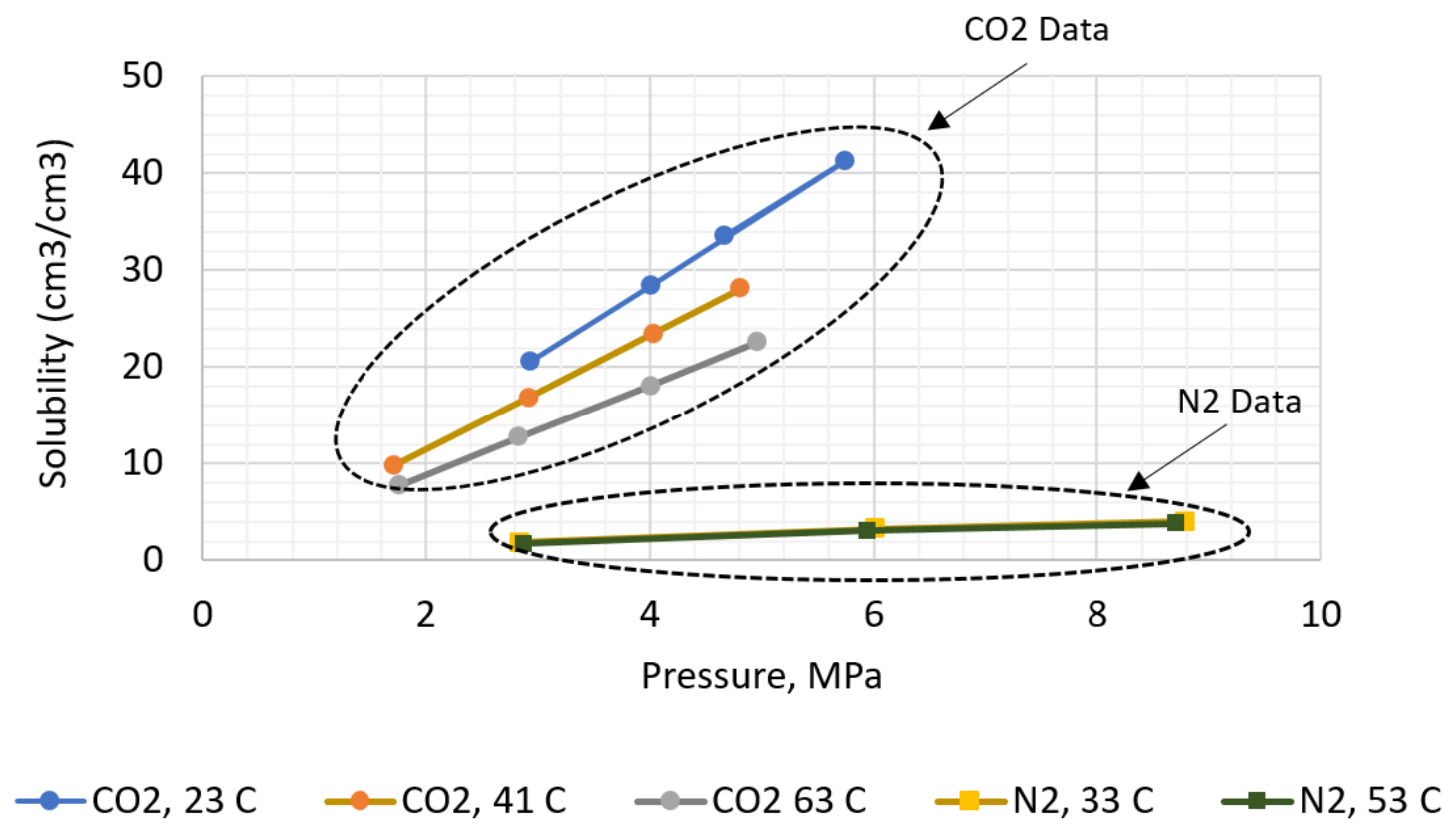

Figure 5.7 Some data points for $\mathrm{CO}_{2}$ and $\mathrm{N}_{2}$ solubility in bitumen, Mehrotra and Svrcek (1982) 
Table 5.1 K-values for carbon dioxide-oil

\begin{tabular}{cccccccccccc}
$\boldsymbol{T}\left({ }^{\circ} \boldsymbol{C}\right)$ & \multicolumn{10}{c}{ Pressure (kPa) } \\
\cline { 2 - 13 } & $\mathbf{1 0 0}$ & $\mathbf{8 0 0}$ & $\mathbf{1 5 0 0}$ & $\mathbf{2 2 0 0}$ & $\mathbf{2 9 0 0}$ & $\mathbf{3 6 0 0}$ & $\mathbf{4 3 0 0}$ & $\mathbf{5 0 0 0}$ & $\mathbf{5 7 0 0}$ & $\mathbf{6 4 0 0}$ & $\mathbf{7 1 0 0}$ \\
\hline $\boldsymbol{1 1}$ & 57 & 7.4 & 4.2 & 3.0 & 2.4 & 2.0 & 1.8 & 1.8 & 1.8 & 1.8 & 1.8 \\
\hline $\boldsymbol{6 1}$ & 103 & 13.3 & 7.4 & 5.2 & 4.1 & 3.4 & 3.0 & 2.6 & 2.4 & 2.2 & 2.1 \\
\hline $\mathbf{1 1 1}$ & 150 & 19.4 & 10.6 & 7.4 & 5.8 & 4.8 & 4.1 & 3.6 & 3.3 & 3.0 & 2.8 \\
\hline $\mathbf{1 6 1}$ & 191 & 24.4 & 13.3 & 9.3 & 7.2 & 5.9 & 5.1 & 4.5 & 4.0 & 3.6 & 3.3 \\
\hline $\mathbf{2 1 1}$ & 220 & 28.0 & 15.2 & 10.5 & 8.1 & 6.7 & 5.7 & 5.0 & 4.5 & 4.0 & 3.7 \\
\hline $\mathbf{2 6 1}$ & 237 & 30.1 & 16.3 & 11.3 & 8.7 & 7.2 & 6.1 & 5.0 & 4.5 & 4.0 & 3.7
\end{tabular}

Table 5.2 K-values for propane-oil

\begin{tabular}{cccccccccccc}
$\boldsymbol{T}\left({ }^{\circ} \boldsymbol{C}\right)$ & \multicolumn{10}{c}{ Pressure $(\mathbf{k P a})$} \\
\cline { 2 - 12 } & $\mathbf{1 0 0}$ & $\mathbf{8 0 0}$ & $\mathbf{1 5 0 0}$ & $\mathbf{2 2 0 0}$ & $\mathbf{2 9 0 0}$ & $\mathbf{3 6 0 0}$ & $\mathbf{4 3 0 0}$ & $\mathbf{5 0 0 0}$ & $\mathbf{5 7 0 0}$ & $\mathbf{6 4 0 0}$ & $\mathbf{7 1 0 0}$ \\
\hline $\boldsymbol{1 1}$ & 6 & 1.20 & 0.71 & 0.97 & 1.20 & 1.39 & 1.54 & 1.54 & 1.54 & 1.54 & 1.54 \\
\hline $\boldsymbol{6 1}$ & 17 & 2.39 & 1.40 & 1.40 & 1.40 & 1.40 & 1.40 & 1.40 & 1.40 & 1.40 & 1.40 \\
\hline $\mathbf{1 1 1}$ & 35 & 4.79 & 2.76 & 2.02 & 1.64 & 1.41 & 1.26 & 1.26 & 1.26 & 1.26 & 1.26 \\
\hline $\mathbf{1 6 1}$ & 56 & 7.56 & 4.38 & 3.20 & 2.57 & 2.18 & 1.92 & 1.73 & 1.59 & 1.48 & 1.39 \\
\hline $\mathbf{2 1 1}$ & 78 & 10.2 & 5.73 & 4.14 & 3.36 & 2.88 & 2.56 & 2.31 & 2.11 & 1.95 & 1.82 \\
\hline $\mathbf{2 6 1}$ & 97 & 12.6 & 7.02 & 4.97 & 3.91 & 3.27 & 2.83 & 2.51 & 2.33 & 2.19 & 2.09
\end{tabular}

Table 5.3 K-values for methane-oil

\begin{tabular}{cccccccccccc}
$\boldsymbol{T}\left({ }^{\circ} \boldsymbol{C}\right)$ & \multicolumn{10}{c}{ Pressure $(\mathbf{k P a})$} \\
\cline { 2 - 12 } & $\mathbf{1 0 0}$ & $\mathbf{8 0 0}$ & $\mathbf{1 5 0 0}$ & $\mathbf{2 2 0 0}$ & $\mathbf{2 9 0 0}$ & $\mathbf{3 6 0 0}$ & $\mathbf{4 3 0 0}$ & $\mathbf{5 0 0 0}$ & $\mathbf{5 7 0 0}$ & $\mathbf{6 4 0 0}$ & $\mathbf{7 1 0 0}$ \\
\hline $\mathbf{1 1}$ & 144 & 18.6 & 10.2 & 7.2 & 5.60 & 4.7 & 4.0 & 3.6 & 3.2 & 2.9 & 2.7 \\
\hline $\boldsymbol{6 1}$ & 202 & 25.8 & 14.1 & 9.8 & 7.60 & 6.3 & 5.4 & 4.7 & 4.2 & 3.8 & 3.5 \\
\hline $\mathbf{1 1 1}$ & 245 & 31.6 & 17.2 & 11.9 & 9.20 & 7.6 & 6.4 & 5.6 & 5.0 & 4.6 & 4.2 \\
\hline $\mathbf{1 6 1}$ & 273 & 35.0 & 19.2 & 13.3 & 10.3 & 8.4 & 7.2 & 6.3 & 5.6 & 5.0 & 4.6 \\
\hline $\mathbf{2 1 1}$ & 287 & 36.5 & 19.8 & 13.6 & 10.5 & 8.7 & 7.4 & 6.5 & 5.8 & 5.2 & 4.8 \\
\hline $\mathbf{2 6 1}$ & 288 & 36.5 & 19.8 & 13.7 & 10.6 & 8.6 & 7.3 & 6.0 & 5.3 & 4.8 & 4.5
\end{tabular}


Table 5.4 K-values for nitrogen-oil

\begin{tabular}{cccccccccccc}
$\boldsymbol{T}\left({ }^{\circ} \boldsymbol{C}\right)$ & \multicolumn{10}{c}{ Pressure (kPa) } \\
\cline { 2 - 12 } & $\mathbf{1 0 0}$ & $\mathbf{8 0 0}$ & $\mathbf{1 5 0 0}$ & $\mathbf{2 2 0 0}$ & $\mathbf{2 9 0 0}$ & $\mathbf{3 6 0 0}$ & $\mathbf{4 3 0 0}$ & $\mathbf{5 0 0 0}$ & $\mathbf{5 7 0 0}$ & $\mathbf{6 4 0 0}$ & $\mathbf{7 1 0 0}$ \\
\hline $\boldsymbol{1 1}$ & 413 & 52.6 & 28.5 & 19.7 & 15.2 & 12.5 & 10.6 & 9.3 & 8.2 & 7.4 & 6.8 \\
\hline $\boldsymbol{6 1}$ & 476 & 60.4 & 32.6 & 22.5 & 17.3 & 14.1 & 12.0 & 10.4 & 9.3 & 8.4 & 7.6 \\
\hline $\boldsymbol{1 1 1}$ & 502 & 64.0 & 34.6 & 23.9 & 18.3 & 14.9 & 12.6 & 11.0 & 9.7 & 8.8 & 8.0 \\
\hline $\mathbf{1 6 1}$ & 501 & 63.5 & 34.5 & 23.8 & 18.3 & 14.9 & 12.7 & 11.0 & 9.8 & 8.8 & 8.0 \\
\hline $\boldsymbol{2 1 1}$ & 482 & 61.0 & 32.9 & 22.2 & 17.1 & 14.0 & 11.9 & 10.4 & 9.2 & 8.3 & 7.6 \\
\hline $\mathbf{2 6 1}$ & 450 & 56.9 & 30.7 & 21.2 & 16.3 & 13.3 & 11.3 & 8.8 & 7.8 & 7.1 & 6.5
\end{tabular}

\subsection{Reservoir Model}

CMG's STARS is an advanced process reservoir simulator that offers many options to model enhanced oil recovery processes, including thermal processes like SAGD or hybrid SAGD. It was originally developed to simulate steam flooding, steam cycling, and steam with additives, along with many types of chemical EOR processes using a wide range of grid and porosity models at both the field and laboratory scale.

In this software, through the main interface "Builder," a conservation equation is constructed for each component of a set of components that describes all the fluids of interest. It adopts the finite volume approach, and all the equations are based on region of interest with volume " $V$ " divided into a grid. Depending on the co-ordinate system selected, the gridblocks are defined accordingly. For example, for a Cartesian coordinate system, the gridblocks are defined for all three spatial coordinates, $x, y$ and $z$.

In each gridblock there are volumes of interest, for example, bulk volume $\left(V_{\mathrm{b}}\right)$, rock volume $\left(V_{\mathrm{r}}\right)$, void volume $\left(V_{\mathrm{v}}\right)$, solid volume $\left(V_{\mathrm{s}}\right)$, volume of fluid phases added together $\left(V_{\mathrm{f}}\right)$, water phase volume $\left(V_{\mathrm{w}}\right)$, oil phase volume $\left(V_{\mathrm{o}}\right)$, gas phase volume $\left(V_{\mathrm{g}}\right)$, etc. Using the appropriate volume ratio, the saturation of each phase is defined, for example: 
$S_{\mathrm{w}}=\frac{V_{\mathrm{w}}}{V_{\mathrm{f}}}$

$S_{\mathrm{o}}=\frac{V_{0}}{V_{\mathrm{f}}}$

$S_{\mathrm{g}}=\frac{V_{\mathrm{g}}}{V_{\mathrm{f}}}$

$S_{\mathrm{w}}+S_{\mathrm{o}}+S_{\mathrm{g}}=1$

where

$S_{\mathrm{g}}=$ gas saturation

$S_{\mathrm{o}}=$ oil saturation

$S_{\mathrm{w}}=$ water saturation

For each gridblock, the conservation equations are defined both for the accumulation and flow terms (CMG STARS User Guide). The governing equations are solved simultaneously for each gridblock. Aziz and Settari (1979) presented a general review of the flow equations solution methods. Equations are solved simultaneously, using Newton's method, in a generalized form which can handle many coupled equations. The fundamental equations for STARS are presented below.

\section{STARS Fundamental Equations}

In CMG's STARS software, for each gridblock the conservation equations are defined both for the accumulation and flow terms. For example, the accumulation term for a flowing and adsorbed component " $i$ " is

$\frac{\partial}{\partial t}\left[V_{\mathrm{f}}\left(\rho_{\mathrm{w}} S_{\mathrm{w}} w_{i}+\rho_{\mathrm{o}} S_{\mathrm{o}} x_{i}+\rho_{\mathrm{g}} S_{\mathrm{g}} y_{i}\right)+V_{\mathrm{v}} A d_{i}\right]$ 
The accumulation term for energy is

$\frac{\partial}{\partial t}\left[V_{\mathrm{f}}\left(\rho_{\mathrm{w}} S_{\mathrm{w}} U_{\mathrm{w}}+\rho_{\mathrm{o}} S_{\mathrm{o}} U_{\mathrm{o}}+\rho_{\mathrm{g}} S_{\mathrm{g}} U_{\mathrm{g}}\right)+V_{\mathrm{v}} c_{\mathrm{s}} U_{\mathrm{s}}+V_{\mathrm{r}} U_{\mathrm{r}}\right]$

The flow term of flow component " $i$ " between two regions is

$\rho_{\mathrm{w}} v_{\mathrm{w}} w_{i}+\rho_{\mathrm{o}} v_{\mathrm{o}} x_{i}+\rho_{\mathrm{g}} v_{\mathrm{g}} y_{i}+\emptyset \rho_{\mathrm{w}} D_{\mathrm{w} i} \nabla w_{i}+\emptyset \rho_{\mathrm{g}} D_{\mathrm{g} i} \nabla y_{i}+\emptyset \rho_{\mathrm{o}} D_{\mathrm{o} i} \nabla x_{i}$

The flow term for energy between two regions is

$\rho_{\mathrm{w}} v_{\mathrm{w}} H_{\mathrm{w}}+\rho_{\mathrm{o}} v_{\mathrm{o}} H_{\mathrm{o}}+\rho_{\mathrm{g}} v_{\mathrm{g}} H_{\mathrm{g}}+K_{\mathrm{T}} \nabla T$

The volumetric flow rates are given by:

$v_{j}=T_{\mathrm{r}}\left[\frac{k_{\mathrm{r} j}}{\mu_{j} r_{j}}\right] \nabla \Phi_{j}$, where $j=\mathrm{w}, \mathrm{o}, \mathrm{g}$

Similarly, the wells source/sink terms for flowing component " $i$ " and energy are defined as:

$\rho_{\mathrm{w}} q_{\mathrm{w} k} w_{i}+\rho_{\mathrm{o}} q_{\mathrm{o} k} x_{i}+\rho_{\mathrm{g}} q_{\mathrm{g} k} y_{i}$

$\rho_{\mathrm{w}} q_{\mathrm{w} k} H_{\mathrm{w}}+\rho_{\mathrm{o}} q_{\mathrm{o} k} H_{\mathrm{o}}+\rho_{\mathrm{g}} q_{\mathrm{g} k} H_{\mathrm{g}}$

The well phase rates are defined as:

$q_{\mathrm{j} k}=I_{j k} \cdot\left(p_{\mathrm{wf} k}-p_{k}\right)$, where $j=\mathrm{w}, \mathrm{o}, \mathrm{g}$

where

$p_{\mathrm{wfk}}=p_{\mathrm{wf}}+\int_{h_{i}}^{h_{k}} \gamma_{\mathrm{av}} \mathrm{gdh}$ 
where $\rho_{j}$ is fluid density $\left(\mathrm{kg} / \mathrm{m}^{3}\right)$ and $j=\mathrm{o}, \mathrm{w}, \mathrm{g}$, and $\mathrm{o}=\mathrm{oil}, \mathrm{w}=$ water, $\mathrm{g}=\mathrm{gas} ; U_{j}$ is internal energy as a function of temperature and phase composition (joules) and $j=\mathrm{o}, \mathrm{w}, \mathrm{g} ; U_{\mathrm{r}}$ is energy per rock volume (joules); $U_{\mathrm{s}}$ is energy per solid volume (joules); $v_{j}$ is volumetric flow rate $\left(\mathrm{m}^{3} / \mathrm{s}\right)$ and $j=$ $\mathrm{o}, \mathrm{w}, \mathrm{g} ; A d_{i}$ is adsorbed concentration $\left(\mathrm{kg} / \mathrm{m}^{3}\right) ; D_{j i}$ is component dispersibilities $\left(\mathrm{m}^{2} / \mathrm{s}\right)$ and $j=$ $\mathrm{o}, \mathrm{w}, \mathrm{g} ; H_{j}$ is enthalpy (joules $/ \mathrm{kg}$ ) and $j=\mathrm{o}, \mathrm{w}, \mathrm{g} ; T_{\mathrm{r}}$ is transmissibility between the two regions; $\mu_{j}$ is viscosity of fluid (cP) and $j=\mathrm{o}, \mathrm{w}, \mathrm{g} ; \nabla \Phi_{j}$ is the value at the node of the adjacent region minus the value at the node of the current region of interest; a positive value for $\nabla \Phi_{j}$ represents inflow, a negative value gives outflow; $w_{i}$ is water phase composition; $x_{i}$ is oil phase composition; $y_{i}$ is gas phase composition; $H_{j}$ is enthalpy (joules/kg) and $j=\mathrm{o}, \mathrm{w}, \mathrm{g} ; h_{k}$ is the elevation of layer $k ; \gamma_{\mathrm{av}}$ is an average mass density of the fluids in the wellbore; $I_{j k}$ is phase $j$ index for well layer $k$, and may be specified in various ways; $p_{k}$ is node pressure of the region of interest which contains well layer $\mathrm{k}$; and $p_{\mathrm{wf} k}$ is flowing wellbore pressure in well layer $k$.

Subscript $k$ refers to the fact that the region of interest contains layer no. $k$ of a well which may be completed also in other blocks or regions.

The heat loss source sink terms are:

$\sum_{k=1}^{n_{f}} H L_{\mathrm{k}}+H L_{\mathrm{v}}+H L_{\mathrm{c}}$

where

$H L_{k}$ is rate of heat transfer to the region of interest through block face number $k$, from the adjacent formation. The heat transfer rate and heat accumulated in the overburden are calculated using an analytical solution for an infinite overburden. Heat flow back into the reservoir block may occur. $H L_{\mathrm{v}}$ is rate of heat transfer calculated from a convective model

$H L_{\mathrm{c}}$ is represents a constant heat transfer model.

The phase mole fractions are calculated from the equilibrium $\mathrm{K}$-value data that is provided through the fluid model, so: 
$y_{i}=K_{\mathrm{i}}^{\mathrm{go}} x_{i}$

$x_{i}=K_{i}^{\mathrm{ow}} w_{i}$

$w_{i}=K_{i}^{\mathrm{wg}} y_{i}$

The phase mole fractions are constrained by:

$\sum_{\mathrm{k}=1}^{n_{\mathrm{c}}} y_{i}=1$ when $S_{\mathrm{g}}>0$

$\sum_{\mathrm{k}=1}^{n_{\mathrm{c}}} x_{i}=1$ when $S_{\mathrm{o}}>0$

$\sum_{k=1}^{n_{\mathrm{c}}} w_{i}=1$ when $S_{\mathrm{w}}>0$

The well equations are coupled to the reservoir conditions by $I_{j k}$ in Eq. 22, that is, the mobility factor $\left(k_{\mathrm{rj}} / \mu_{\mathrm{j}}\right)$. The mole rate $\rho_{\mathrm{w}} q_{\mathrm{w} k} w_{i}+\rho_{\mathrm{o}} q_{\mathrm{o} k} x_{i}+\rho_{\mathrm{g}} q_{\mathrm{g} k} y_{i}$ is evaluated for each component, a flash is performed to obtain phase splits and compositions using surface condition K-values; surface densities are calculated; and surface mole phase rates are multiplied by surface densities to get surface volume rates.

Overall, the spatially discretized conservation equation of flowing component $I$ is:

$$
\begin{aligned}
& \frac{\partial}{\partial t}\left[V_{f}\left(\rho_{\mathrm{w}} S_{\mathrm{w}} w_{i}+\rho_{\mathrm{o}} S_{\mathrm{o}} x_{i}+\rho_{\mathrm{g}} S_{\mathrm{g}} y_{i}\right)+V_{v} A d_{i}\right]=\sum_{\mathrm{k}=1}^{n_{f}}\left[T_{\mathrm{w}} \rho_{\mathrm{w}} w_{i} \nabla \Phi_{\mathrm{w}}+T_{o} \rho_{o} x_{i} \nabla \Phi_{\mathrm{o}}+\right. \\
& \left.T_{\mathrm{g}} \rho_{\mathrm{g}} y_{i} \nabla \Phi_{\mathrm{g}}\right]+V \sum_{\mathrm{k}=1}^{n_{f}}\left[S_{k i}^{\prime}-S_{k i}\right] r_{k}+\sum_{\mathrm{k}=1}^{n_{f}}\left[\varnothing \rho_{\mathrm{w}} D_{\mathrm{wi}} \nabla w_{i}+\emptyset \rho_{\mathrm{o}} D_{\mathrm{oi}} \nabla x_{i}+\emptyset \rho_{\mathrm{g}} D_{\mathrm{gi}} \nabla y_{i}\right]+ \\
& \delta_{i \mathrm{w}} \sum_{\mathrm{k}=1}^{n_{\mathrm{f}}} \rho_{\mathrm{w}} q a q_{\mathrm{w} k}+\rho_{\mathrm{w}} q_{\mathrm{w} k} w_{i}+\rho_{\mathrm{o}} q_{\mathrm{o} k} x_{i}+\rho_{\mathrm{g}} q_{\mathrm{g} k} y_{i}
\end{aligned}
$$

Where, $n_{f}$ is the number of neighbouring regions or gridblock faces, $S_{k i}^{\prime}$ is the product stoichiometry coefficient of component $I$ in reaction $k, S_{k i}$ is the reactant stoichiometry coefficient of component $I$ in reaction $k, r_{k}$ is the volumetric rate of reaction $k$, calculated from a model for 
reaction kinetics, $q a q_{\mathrm{w} k}$ is volumetric water flow rate through a block face $k$ to/from the adjacent aquifer.

The conservation equation of solid component is:

$\frac{\partial}{\partial t}\left[V_{\mathrm{v}} c_{i}\right]=V \sum_{\mathrm{k}=1}^{n_{f}}\left[S_{k i}^{\prime}-S_{k i}\right] r_{k}$

The spatially discretized conservation equation of energy is:

$$
\begin{aligned}
& \frac{\partial}{\partial t}\left[V_{f}\left(\rho_{\mathrm{w}} S_{\mathrm{w}} U_{\mathrm{w}}+\rho_{\mathrm{o}} S_{\mathrm{o}} U_{\mathrm{o}}+\rho_{\mathrm{g}} S_{\mathrm{g}} U_{\mathrm{g}}\right)+V_{v} c_{\mathrm{s}} U_{\mathrm{s}}+V_{r} U_{r}\right]=\sum_{\mathrm{k}=1}^{n_{f}}\left[T_{\mathrm{w}} \rho_{\mathrm{w}} H_{\mathrm{w}} \nabla \Phi_{\mathrm{w}}+T_{\mathrm{o}} \rho_{\mathrm{o}} H_{\mathrm{o}} \nabla \Phi_{\mathrm{o}}+\right. \\
& \left.T_{\mathrm{g}} \rho_{\mathrm{g}} H_{\mathrm{g}} \nabla \Phi_{\mathrm{g}}\right]+\sum_{\mathrm{k}=1}^{n_{f}} k \Delta T+\rho_{\mathrm{w}} q_{\mathrm{w} k} H_{w}+\rho_{\mathrm{o}} q_{\mathrm{o} k} H_{\mathrm{o}}+\rho_{\mathrm{g}} q_{\mathrm{g} k} H_{\mathrm{g}}+V \sum_{\mathrm{k}=1}^{n_{\mathrm{f}}} H_{r k} r_{k}+H L_{\mathrm{o}}+H L_{v}+ \\
& H L_{c}+\sum_{\mathrm{k}=1}^{n_{\mathrm{f}}}\left(H A_{C V}+H A_{C D}\right)_{k}
\end{aligned}
$$

where, $H_{r k}$ is the enthalpy of reaction $k, H A_{C V}$ is rate of heat transferred by convection to/from adjacent aquifer, $H A_{C D}$ is rate of heat transferred by conduction to/from adjacent aquifer.

Note that all the equations presented above are taken from CMG STARS User Guide Advanced Processes \& Thermal Reservoir Simulator, version 2016.

The above equations are solved simultaneously for each gridblock. Equations are solved simultaneously, using Newton's method, in a generalized form which can handle many coupled equations. The equations summarized above are written in residual form as

$\mathrm{R}_{\mathrm{i}}=$ [net inflow rate] + [net source/sink rate] - [rate of change of accumulation] and the equation is solved when $R_{i}=0$.

If the total number of equations are denoted by $\mathrm{N}_{\mathrm{eq}}$, and $\mathrm{X}_{\mathrm{i}}$ represents all primary variables, with $\mathrm{i}=1$ to $\mathrm{N}_{\text {eq. }}$. Each residual $\mathrm{R}_{\mathrm{i}}$ is written as:

$R=R(X)$ 


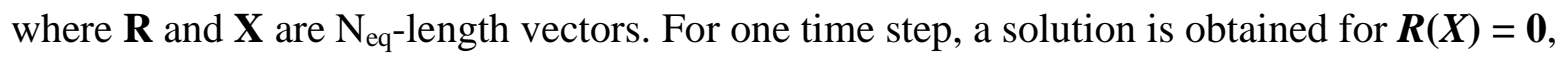
Newton's method, which is written as:

$X^{\mathrm{k}+1}=X^{\mathrm{k}}-\left[J^{\mathrm{k}}\right]^{-1} \cdot \boldsymbol{R}^{\mathrm{k}}$

where $\boldsymbol{J}=\mathrm{d} \boldsymbol{R} / \mathrm{d} \boldsymbol{X}$ is the Jacobian matrix of derivatives and $\mathrm{k}$ is the Newton iteration number.

The entries in the Jacobian are

$J_{i j}=\frac{\partial R_{i}}{\partial X_{j}} \quad i=1$ to $N_{\mathrm{eq}}, j=1$ to $N_{\mathrm{eq}}$

The non-zero Jacobian entries are estimated using numerical differentiation:

$J_{i j} \cong \frac{R_{i}\left(X+\delta X_{j}\right)-R_{i}(X)}{\delta X_{j}}$

STARS uses the Adaptive-Implicit Method (Oballa et al., 1989), and the gridblocks that are experiencing large throughputs or rapid changes in primary variables (pressure, saturation, temperature, etc.) are treated fully implicitly.

\section{Model Assumptions and Justifications}

To simulate each experiment, first a numerical model was formulated. Cartesian coordinates were selected to represent the physical model with gridblocks defined in $i, j$ and $k$ directions. Since the physical model was cylindrical, the corners of the rectangular blocks were cut to represent a cylindrical model. Although STARS offers a radial coordinate system, the flow direction for the STARS radial coordinate system does not agree with the flow direction in the physical model experiments. Furthermore, the radial coordinate system cannot capture the counter current flow. 
The gridblocks were defined such that they represent the shell of the cylindrical model as well as the bulk volume filled with porous matrix. A value of zero porosity was assigned to the gridblocks representing the shell (Figure 5.8).

The gridblock size and distribution in $i, j$ and $k$ directions are presented below:

i direction: number of gridblocks $=30$; grid block size $=1.86 \mathrm{~cm} ; \mathrm{j} \& \mathrm{k}$ direction: number of gridblocks $=13$; gridblock size $($ from left to right $)=0.26 \times 2,0.708 \times 1,1.309 \times 7,0.708 \times 1,0.264 \times 2$.

\section{Porosity 2016-11-29 I layer: 1}

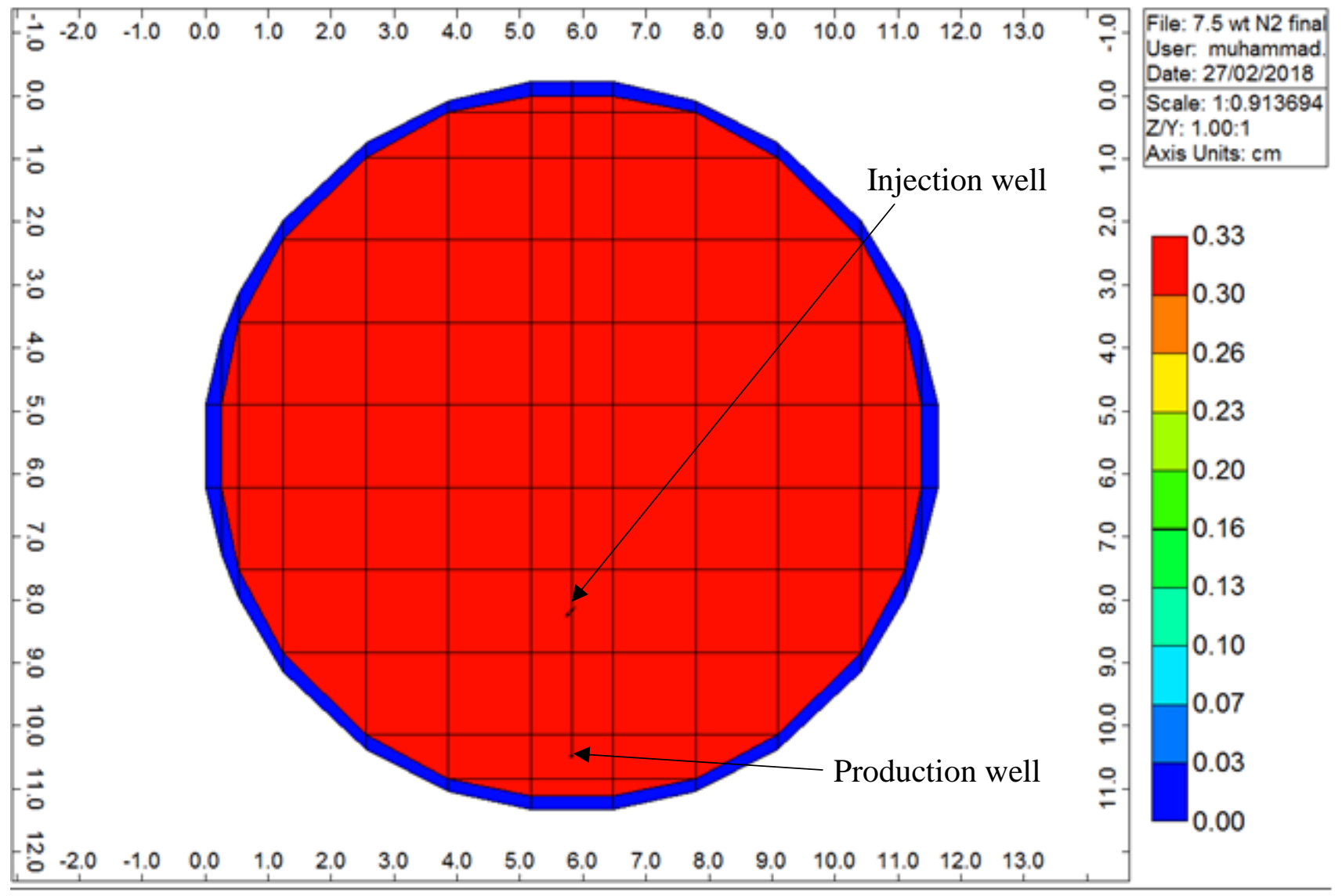

Figure 5.8 Gridblock distribution and porosity - Model side view

The gridblock size was selected such that they were not too small to result in large computational time and still large enough to maintain the resolution and result accuracy. Figure 5.9 presents the 
3D view of the numerical model gridblock distribution. The scale bar in the figure represent the vertical thickness of the model in $\mathrm{cm}$.

The injection and production wells were defined with a configuration similar to that of the physical model. The horizontal length of these wells was along the i direction. Both wells were perforated in each gridblock along the $i$ direction.

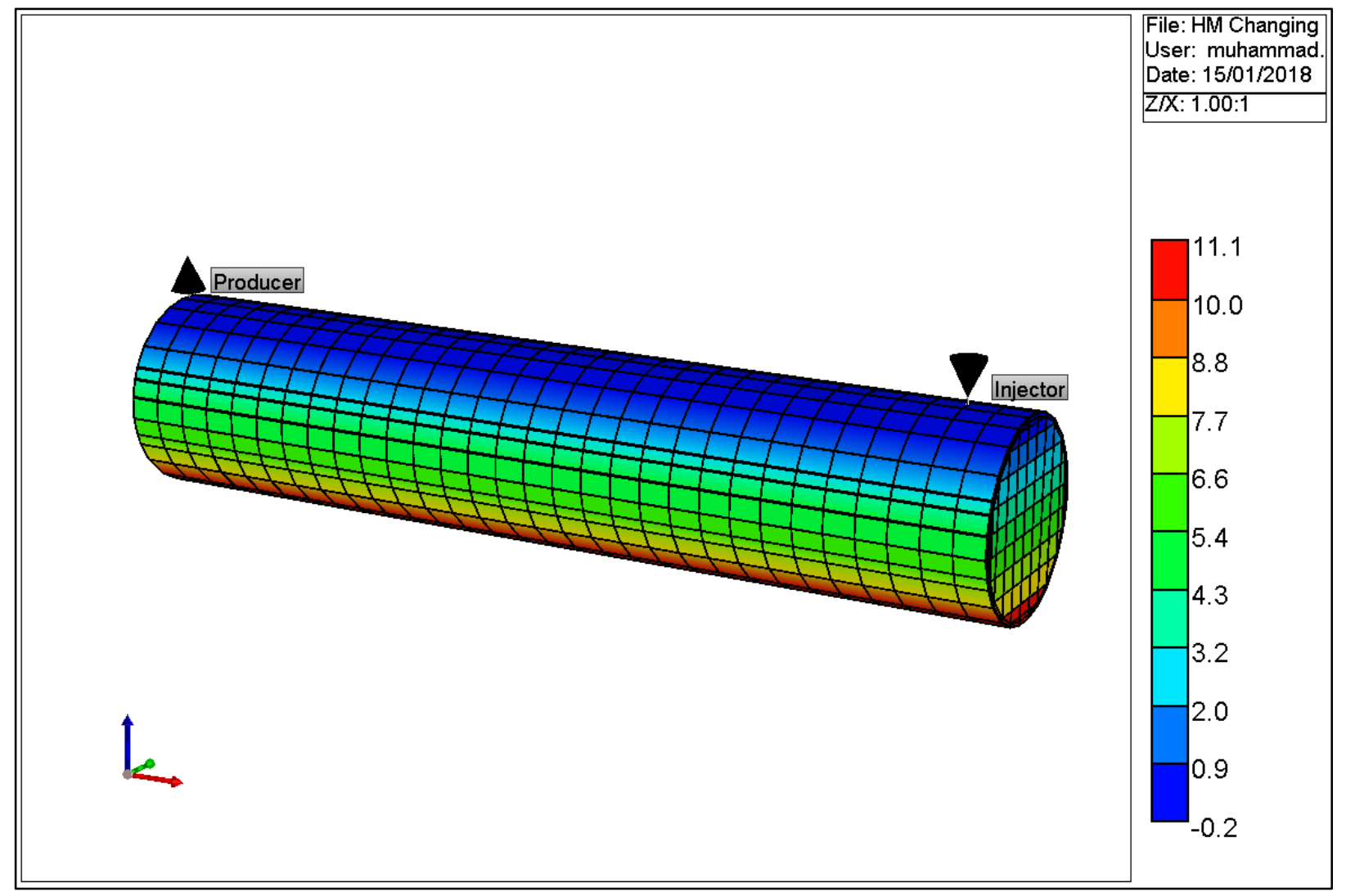

Figure 5.9 Gridblock distribution - Model 3D view

\section{Model Initial Conditions:}

The model was initialized by providing the following information:
$\checkmark$ Matrix permeability and porosity
$\checkmark$ Initial model temperature and pressure
$\checkmark$ Initial oil, water and gas saturation 


\footnotetext{
$\checkmark$ Matrix compressibility

$\checkmark$ Thermal conductivities of fluids and synthetic sand

$\checkmark$ Solvent solubility in terms of equilibrium k-values

$\checkmark$ Oil viscosity as a function of temperature

$\checkmark$ Volume shift and binary interaction coefficient for EOS

$\checkmark$ Starting point for oil-water and oil-gas relative permeability curves

$\checkmark$ Injected fluid type and mole percentage ratio

$\checkmark$ Injected fluid injection rate, pressure and temperature

$\checkmark$ Produced fluid pressure and production rate
}

\section{Model Boundary Conditions:}

Since it was assumed that there are no heat losses from the model, the heat flow from the top and bottom of the model was zero. Also, the heat and mass flow at the boundaries were assumed to be zero.

CMG STARS allows modelling of the heating process by placing a heater in the gridblock of interest. In the numerical modelling reported here, the heaters were placed in the gridblocks representing the model shell, and a constant heat transfer rate was provided to the gridblocks, such that this configuration matched the temperature profile of the external band heaters obtained from an actual experiment. Likewise, the pre-heating stage of the experiments was modeled with heaters.

The following values for matrix compressibility and thermal properties were used:

Matrix compressibility $=1 \times 10^{-4} 1 / \mathrm{kPa}$

Sand volumetric heat capacity $=2.3 \mathrm{~J} / \mathrm{cm}^{3}{ }^{\circ} \mathrm{C}$

Sand thermal conductivity $=1.875 \mathrm{~J} / \mathrm{cm} \min { }^{\circ} \mathrm{C}$

Water phase thermal conductivity $=0.375 \mathrm{~J} / \mathrm{cm} \mathrm{min}{ }^{\circ} \mathrm{C}$

Oil phase thermal conductivity $=0.083 \mathrm{~J} / \mathrm{cm} \min { }^{\circ} \mathrm{C}$

Gas phase thermal conductivity $=0.027 \mathrm{~J} / \mathrm{cm} \min { }^{\circ} \mathrm{C}$ 


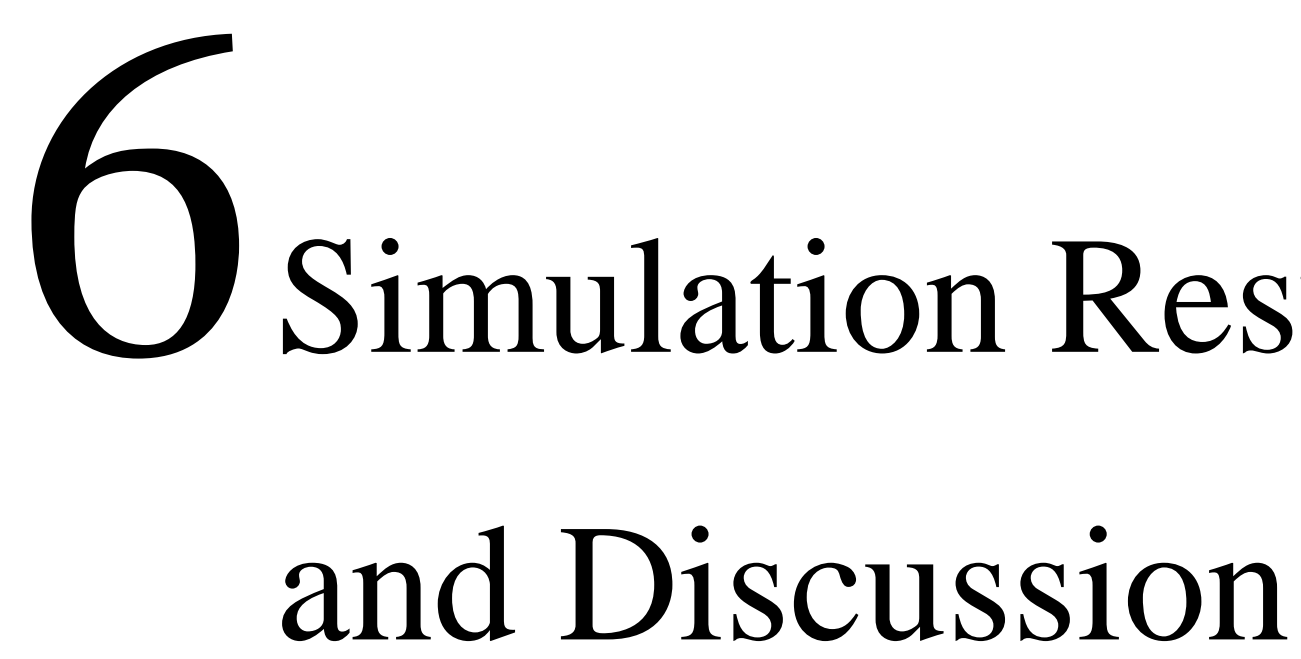

This section of the dissertation presents the history-matched results of solvents that were found to be promising. The simulated oil, water and gas recovery is history-matched for the experiments in which carbon dioxide, nitrogen and methane were added to the injected stream.

Considering both the oil recovery and solvent retention, the experimental study showed that propane is the least attractive solvent for low-pressure hybrid SAGD process. Therefore, propane experiment history matching was excluded from this study. The solvent retention of propane was up to $15 \%$, whereas for carbon dioxide, nitrogen and methane the retention was up to $6 \%$. The solvent retention is an important parameter that dictates the hybrid SAGD process economics. A higher solvent retention corresponds to higher solvent losses in the reservoir and more solvent loading to maintain the injected stream concentration.

The history-match was achieved by changing the relative permeability curves. Some simulations were also performed to investigate the process's sensitivity to solvent dispersion as a variable. 


\subsection{History-Match Results for Steam plus Carbon Dioxide Experiments}

The biggest challenge in conducting the numerical simulation study was to obtain a tuned model for a given solvent, such that it could reasonably predict the results for the same solvent at different injection conditions. The carbon dioxide addition experiments were performed at three different concentrations of 5.0, 7.5 and $10.0 \mathrm{wt} \%$. At first, an effort was made to history-match the results for all three concentrations with a single set of relative permeability curves. This effort was not successful, since the oil recovery was significantly different for the 5.0 and $10.0 \mathrm{wt} \%$ experiments; furthermore, the oil recovery declined with an increase in the carbon dioxide concentration in the injected feed.

Another attempt was made by first history-matching the 5.0 and $10.0 \mathrm{wt} \%$ experiments with unique sets of relative permeability curves, and then devising a single set of curves by averaging the two sets of curves. Using the average relative permeability data, a history-match was attempted for each experiment using solvent dispersion as a history-matching parameter. Even by varying the solvent dispersion by five orders of magnitude, a reasonable history-match of all the experiments was not achieved.

The reason why the model was found to be insensitive to solvent dispersion is that this parameter is a mass transfer property. The higher the solvent dispersion, the higher the solvent mass transfer rate will be, but it is still limited by maximum solvent solubility. The solubilisation of the solvents in the oil phase results in oil viscosity reduction. For non-thermal processes, this reduced viscosity could be many times lower than that of the original dead oil and can have significant positive effect on oil production. For thermal processes, especially at high temperatures, e.g., at $195^{\circ} \mathrm{C}$, the additional viscosity reduction would not be much. Furthermore, generally at high temperatures, the solubility of gases tends to decrease. So, in this scenario, if it is assumed that with the provided dispersion information the solvent solubility reached its maximum concentration at the experimental conditions, it was not enough to significantly affect the results with different solvent concentrations. 
Finally, to attain a tuned numerical model for each concentration of solvent, the relative permeability curves were altered and the injection and production data were history-matched. Since the tests with $7.5 \mathrm{wt} \%$ and $10.0 \mathrm{wt} \%$ carbon dioxide addition resulted in similar oil recovery, the set of relative permeability curves was the same for these two tests.

Table 6.1 shows the parameters that were adjusted to match the experimental data. Using Corey's correlation (Corey, 1954; Corey and Rathjens, 1956; Brooks and Corey, 1964), and the parameters listed in Table 6.1, the simulator generates the relative permeability curves.

Table 6.1 History matching parameters

1 End point saturation connate water - SWCON

2 End point saturation critical water - SWCRIT

3 End point saturation irreducible oil for water oil table - SOIRW

4 End point saturation residual oil for water oil table - SORW

5 End point saturation irreducible oil for gas liquid table - SOIRG

6 End point saturation residual oil for gas liquid table - SORG

7 End point saturation connate gas - SGCON

8 End point saturation critical gas - SGCRIT

$9 \mathrm{~K}_{\mathrm{ro}}$ at connate water - KROCW

$10 \mathrm{~K}_{\mathrm{rw}}$ at irreducible oil - KRWIRO

$11 \mathrm{~K}_{\mathrm{rg}}$ at connate liquid $-\mathrm{KRGCL}$

$12 \mathrm{~K}_{\mathrm{rog}}$ at connate gas - KROGCG

13 Exponent for calculating $\mathrm{k}_{\mathrm{rw}}$ from KRWIRO

14 Exponent for calculating $\mathrm{k}_{\text {row }}$ from KROCW

15 Exponent for calculating $\mathrm{k}_{\mathrm{rog}}$ from KROGCG

16 Exponent for calculating $\mathrm{k}_{\mathrm{rg}}$ from KRGCL

For each experiment, hundreds of simulations were run to match the injection and production data. The history-matched results with corresponding relative permeability curves are presented in this dissertation. Figure 6.1 shows the example of many simulation cases executed with varying the parameters showed in Table 6.1 to obtain a reasonable history match. The blue dots are the 
experimental data. The red line is the reasonable history-match and the black line is the curve obtained with the initial guess provided for the parameters listed in Table 6.1.

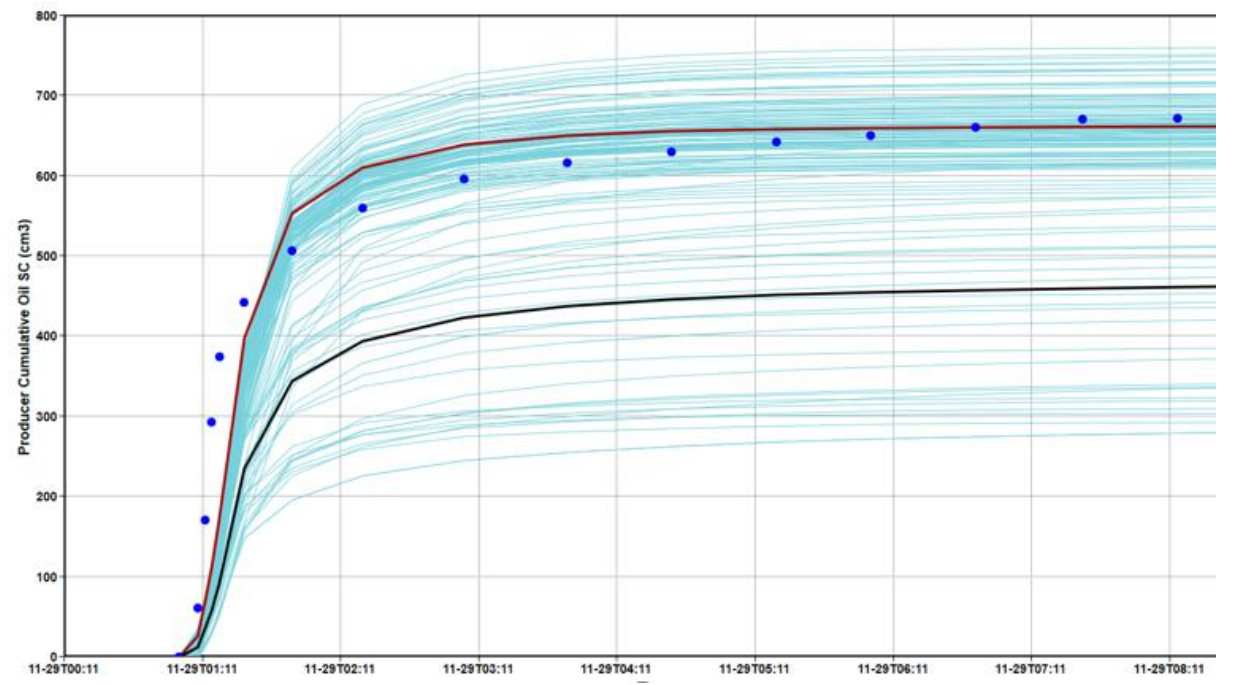

Figure 6.1 History-matching example

Figure 6.2 presents the experimental and history-matched oil production results for the $5.0 \mathrm{wt} \%$ carbon dioxide experiment. The produced oil is along the y-axis; time in hours is along the x-axis. The cumulative root mean square, relative error and standard deviation are shown on the chart.

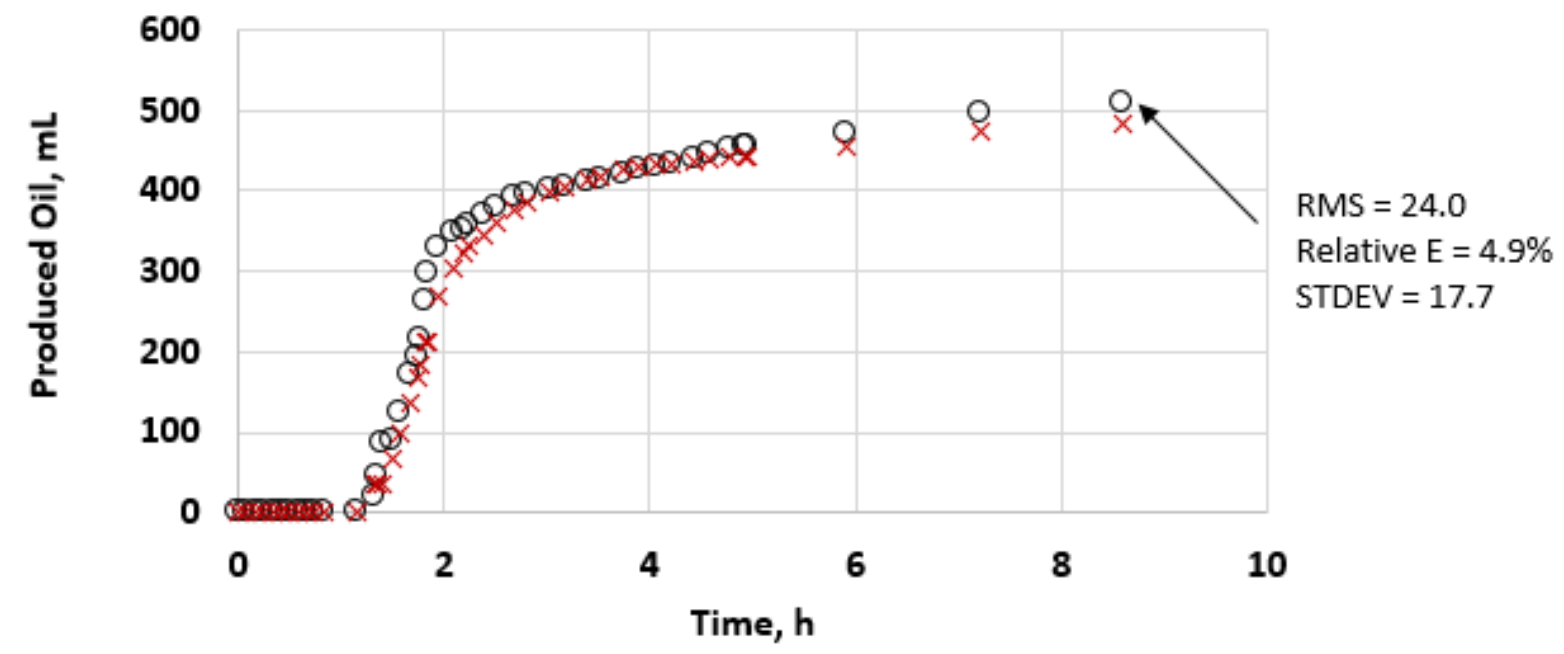

O Experimental

$\times$ Simulated

Figure 6.2 Steam plus $5.0 \mathrm{wt} \% \mathrm{CO}_{2}-$ Oil production history-match 
A positive value of relative error refers to underprediction, and a negative value refers to overprediction. The relative error at the end of production was $4.9 \%$. The simulation slightly underpredicted the produced oil volume. Overall the numerical model reasonably matched the experimental results.

Figure 6.3 presents the experimental and simulated oil production results for the $7.5 \mathrm{wt} \%$ carbon dioxide experiment. Similarly to the $5.0 \mathrm{wt} \%$ simulation, the numerical model underpredicted the oil recovery during the initial injection times. The relative error by the end of production was $4.2 \%$. The simulation slightly underpredicted the produced oil volume.

Figure 6.4 presents the experimental and predicted oil production results for the $10.0 \mathrm{wt} \%$ carbon dioxide experiment. Note that relative permeability curves obtained for the $7.5 \mathrm{wt} \%$ test were used for the $10.0 \mathrm{wt} \%$ test. The numerical model underpredicted the oil recovery during the first two hours. The relative error at the end of production was $-0.4 \%$. The simulation slightly overpredicted the produced oil.

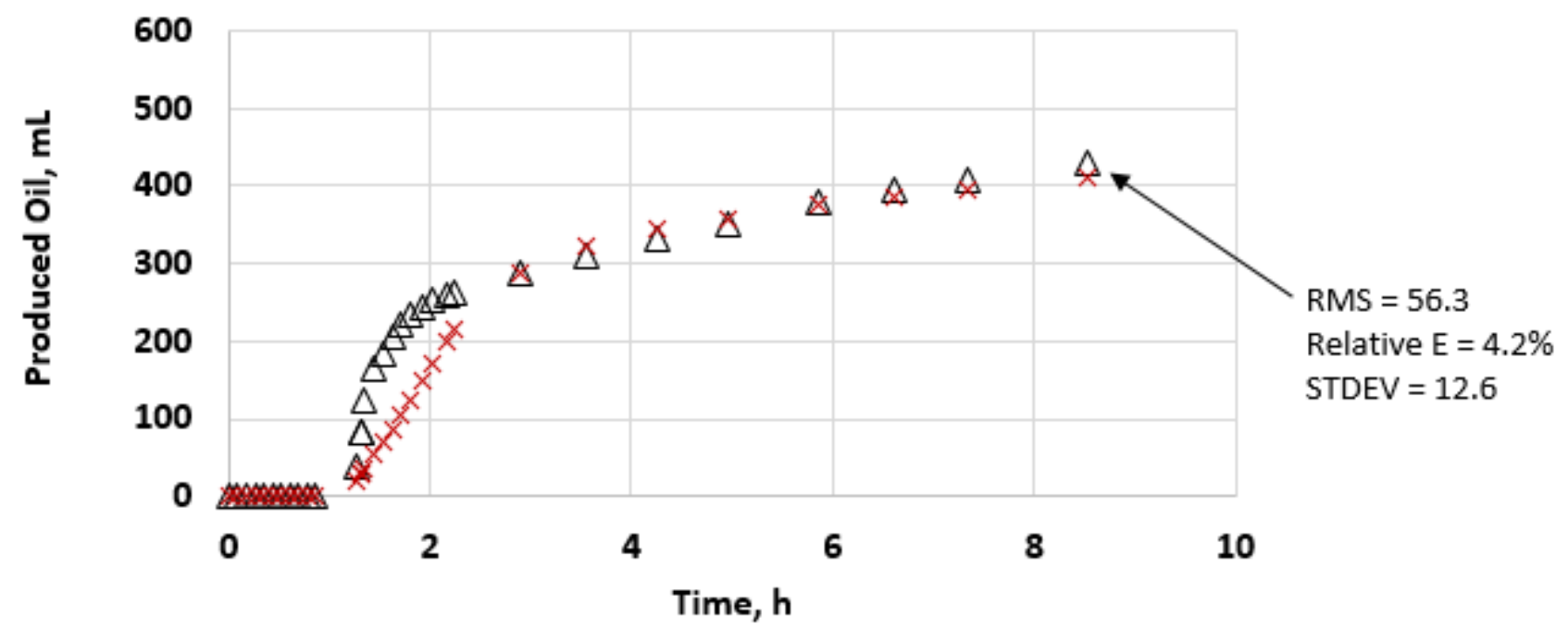

$\triangle$ Experimental

$\times$ Simulated

Figure 6.3 Steam plus $7.5 \mathrm{wt} \% \mathrm{CO}_{2}$ - Oil production history-match 


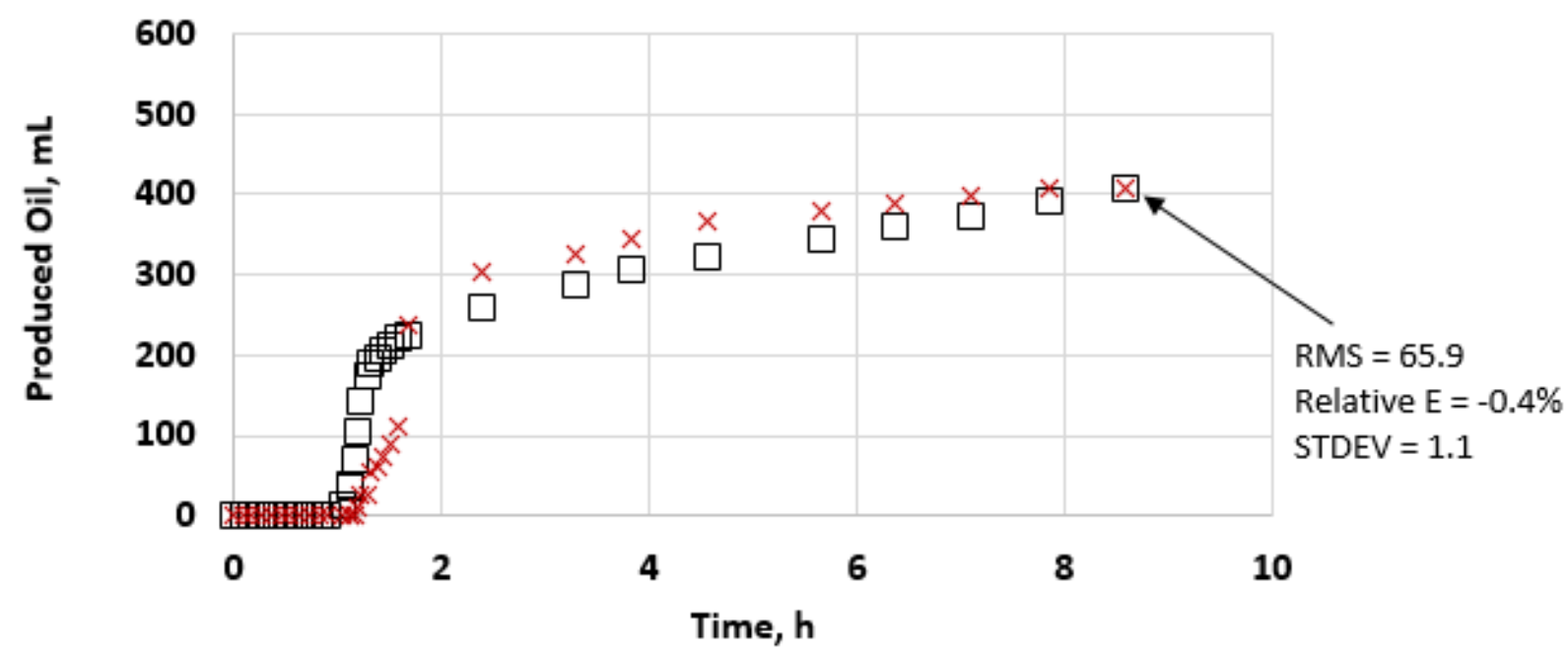

Experimental

$\times$ Simulation

Figure 6.4 Steam plus $10.0 \mathrm{wt} \% \mathrm{CO}_{2}-$ Oil production history-match

Figure 6.5 presents the experimental and simulation results for produced gas for the $5.0 \mathrm{wt} \%$ carbon dioxide experiment. The simulation model showed an early breakthrough of the gas and overpredicted the gas production for most of the experiment. The relative error at the end of production was $-0.4 \%$.

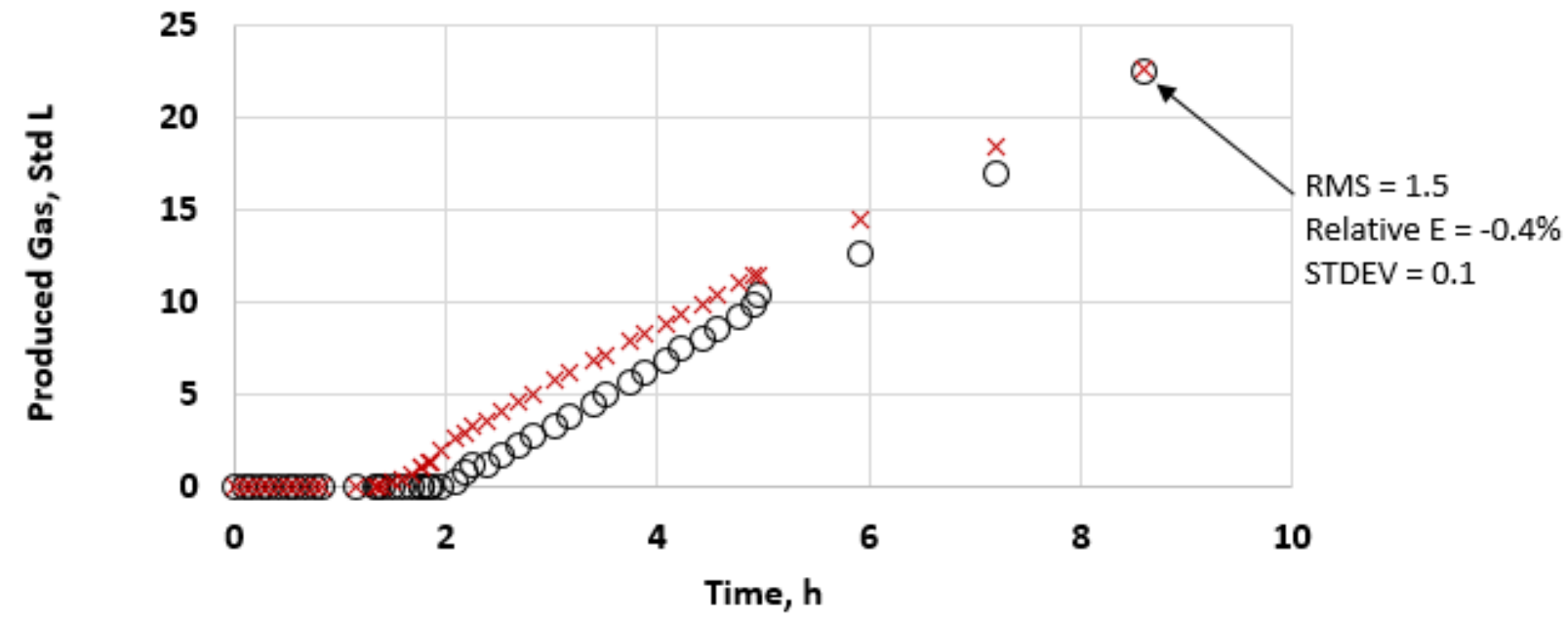

O Experimental

$\times$ Simulated

Figure 6.5 Steam plus $5.0 \mathrm{wt} \% \mathrm{CO}_{2}-$ Gas production history-match 
Figure 6.6 presents the experimental and simulated results for produced gas for the $7.5 \mathrm{wt} \%$ carbon dioxide experiment. Similarly to the previously shown results, the simulated produced gas breakthrough time was overpredicted. Also, the simulation overpredicted the gas production during the initial injection period. Although the error by the end of the production period was $4.7 \%$, overall, the predicted gas production for this experiment was matched better than for the 5.0 wt\% experiment.

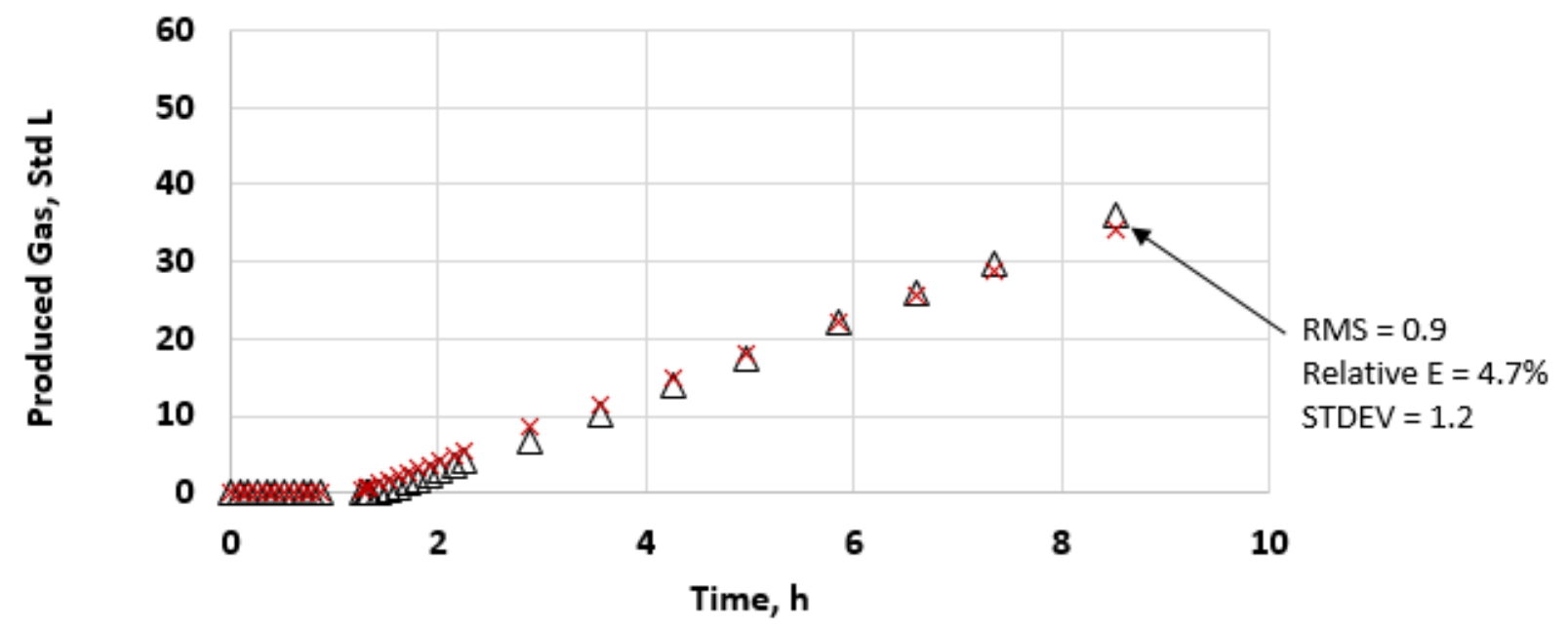

$\Delta$ Experimental

$\times$ Simulated

Figure 6.6 Steam plus $7.5 \mathrm{wt} \% \mathrm{CO}_{2}-$ Gas production history-match

Figure 6.7 presents the experimental and simulated results for produced gas for the $10.0 \mathrm{wt} \%$ carbon dioxide experiment. As in the previously simulated cases, the gas breakthrough time in the simulation model was earlier than the experimental breakthrough time, and the gas production during earlier times was overpredicted. Overall, among all three carbon dioxide experiments, the history-match of produced gas was the best for the $10.0 \mathrm{wt} \%$ run. The error by the end of the production period was $2.1 \%$.

Figure 6.8 presents the experimental and simulated results for produced water for the $5.0 \mathrm{wt} \%$ carbon dioxide experiment. The initial production period, right at the start, was not matched well. After 2 hours into production, the produced water was matched reasonably well. The cumulative 
produced water was slightly overpredicted, with a relative error of $-6.6 \%$ at the end of the production period.

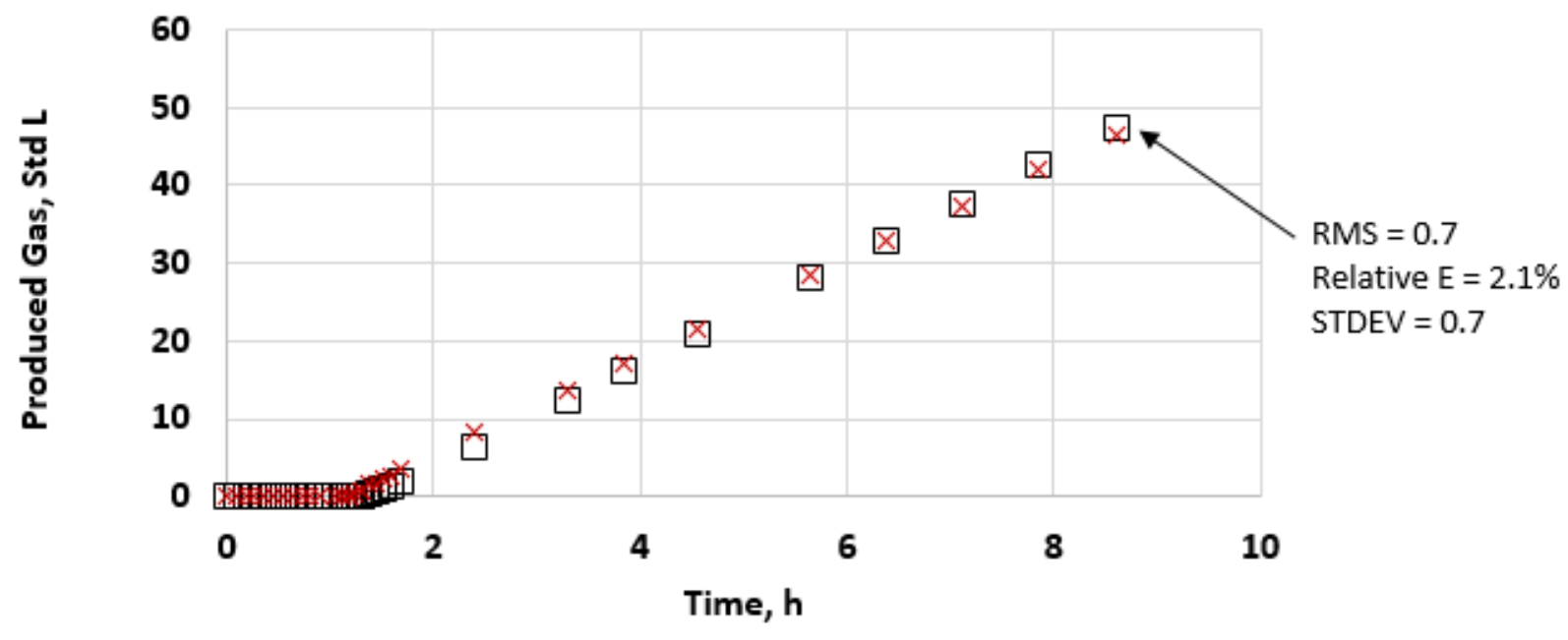

$\square$ Experimental

$\times$ Simulation

Figure 6.7 Steam plus $10.0 \mathrm{wt} \% \mathrm{CO}_{2}-$ Gas production history-match

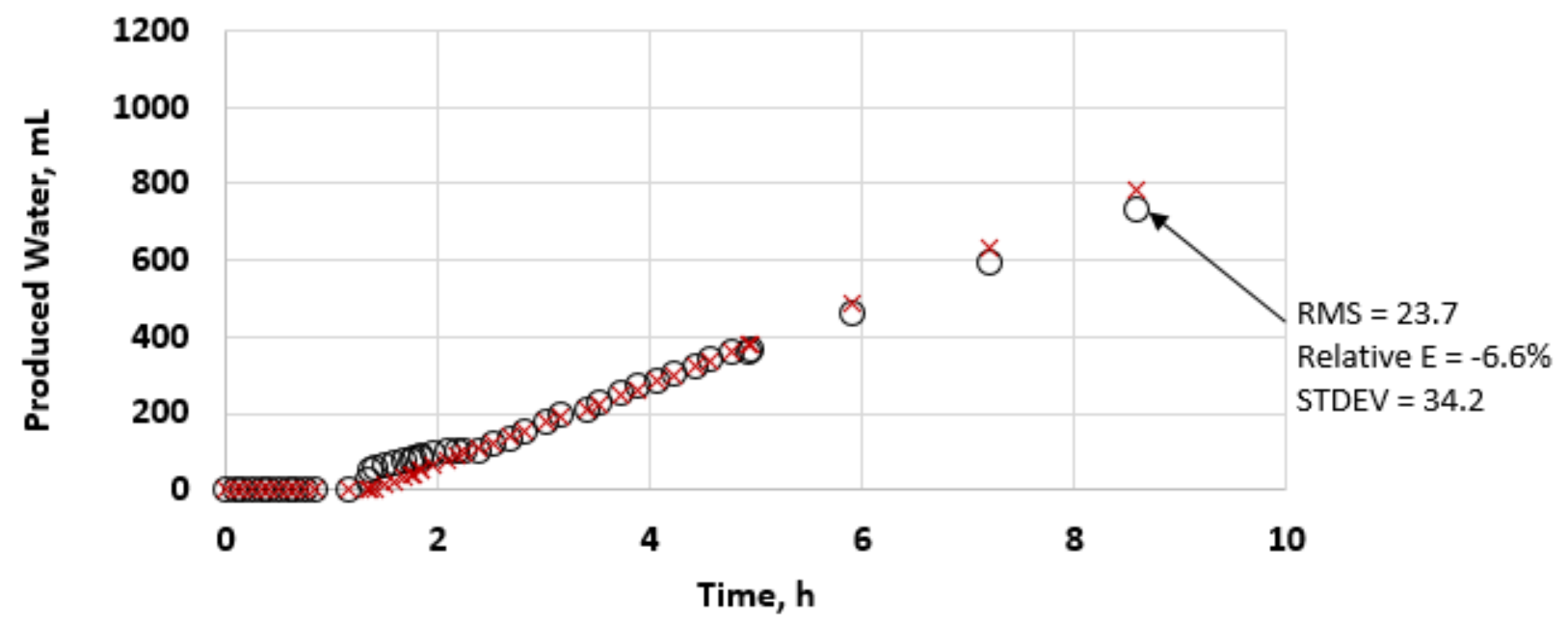

O Experimental

$\times$ Simulated

Figure 6.8 Steam plus 5.0 wt $\% \mathrm{CO}_{2}-$ Water production history-match 
Figure 6.9 presents the experimental and simulated results for produced water for the $7.5 \mathrm{wt} \%$ carbon dioxide experiment. Similarly to the $5.0 \mathrm{wt} \%$ experiment, a good prediction was not achieved for the initial production period. Overall the predictions were close to the experimental results, with an error of $0.9 \%$ at the end of the production period.

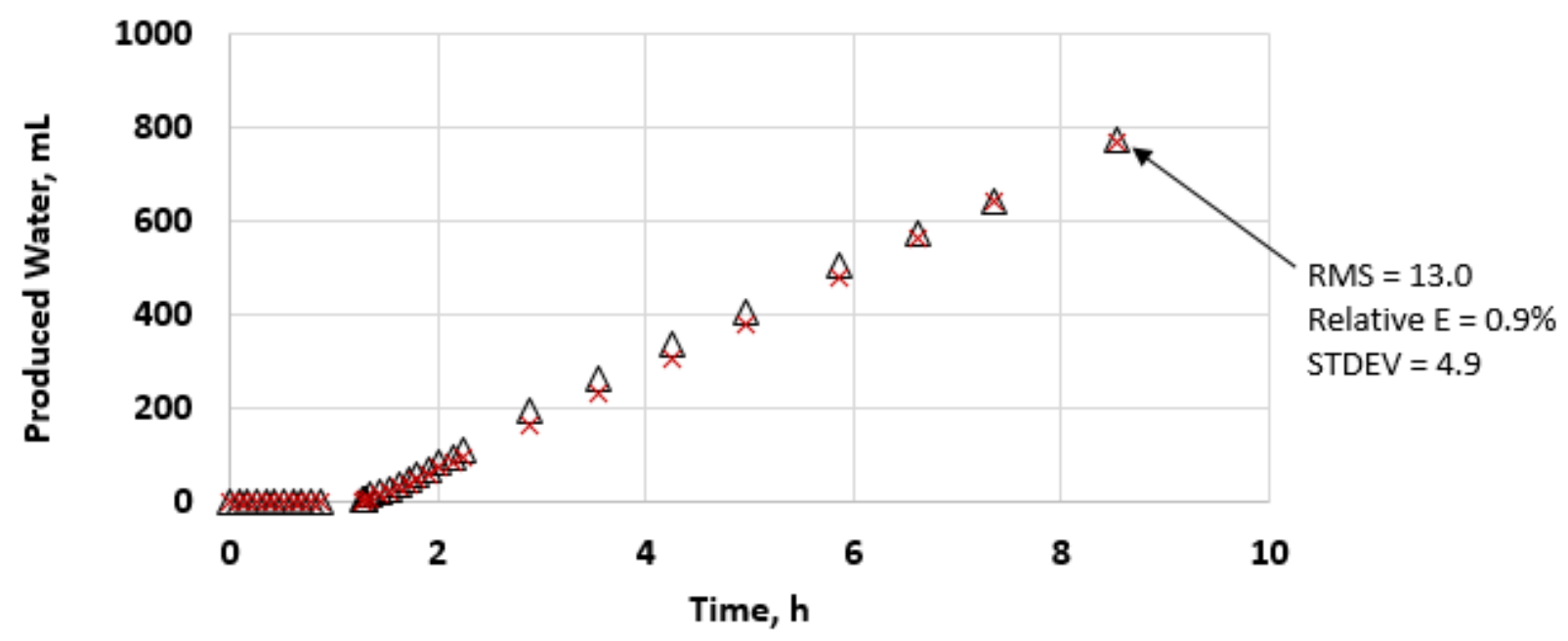

$\Delta$ Experimental

$\times$ Simulation

Figure 6.9 Steam plus $7.5 \mathrm{wt} \% \mathrm{CO}_{2}-$ Water production history-match

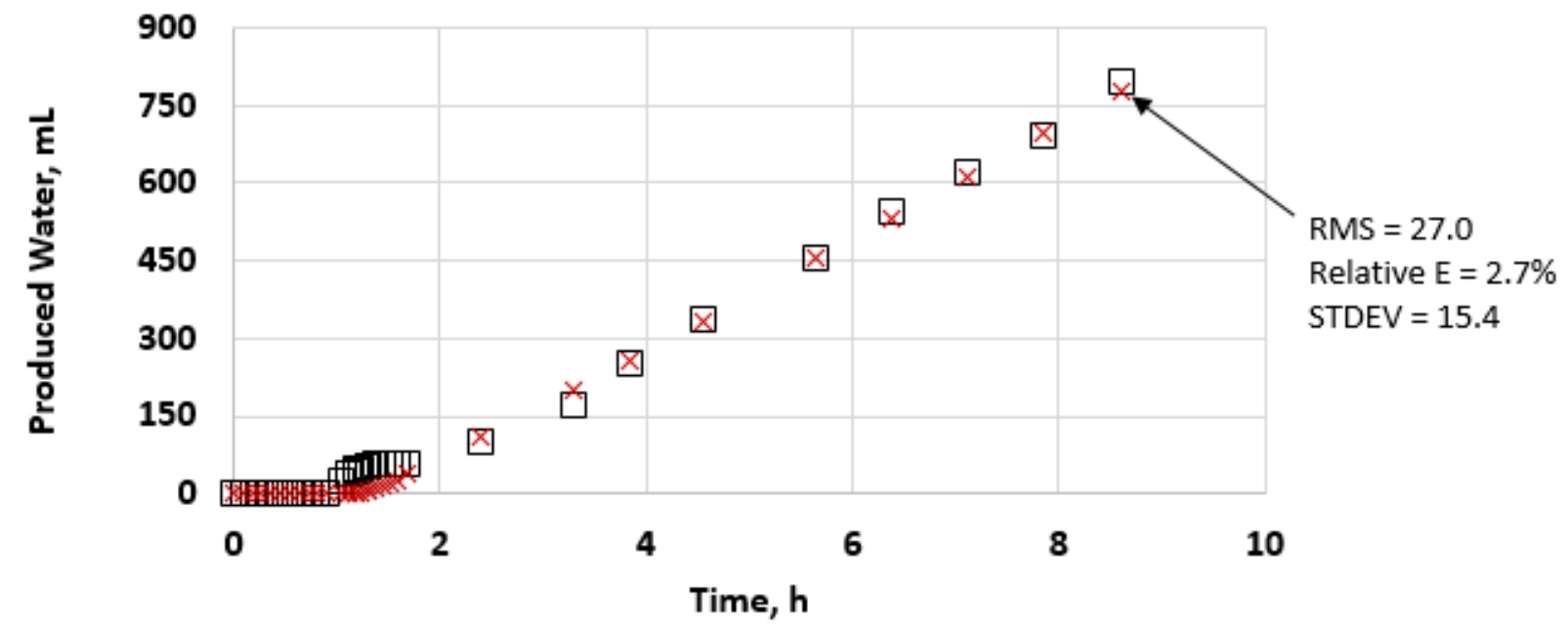

$\square$ Experimental

$\times$ Simulation

Figure 6.10 Steam plus $10.0 \mathrm{wt} \% \mathrm{CO}_{2}-$ Water production history-match 
Figure 6.10 presents the experimental and history-matched results for produced water for the $10.0 \mathrm{wt} \%$ carbon dioxide experiment. The experimental data after 3 hours of production were matched very well. The error at the end of the production was $2.7 \%$.

Figure 6.11 presents the tuned relative permeability curves for oil and water. Figure 6.12 presents the same for oil and gas. The dotted line corresponds to the $5.0 \mathrm{wt} \%$ experiment and the solid lines correspond to the $10.0 \mathrm{wt} \%$ experiment. The oil-water curves are plotted against water saturation, and the oil-gas curves are plotted against liquid saturation or one minus gas saturation. To match the $10.0 \mathrm{wt} \%$ experiment, the $\mathrm{k}_{\text {row }}$ and $\mathrm{k}_{\text {rog }}$ had to be depressed. The relative permeability to water was more effective in adjusting the produced oil. Note that the relative permeability curves for the 10.0 and $7.5 \mathrm{wt} \%$ tests were the same.

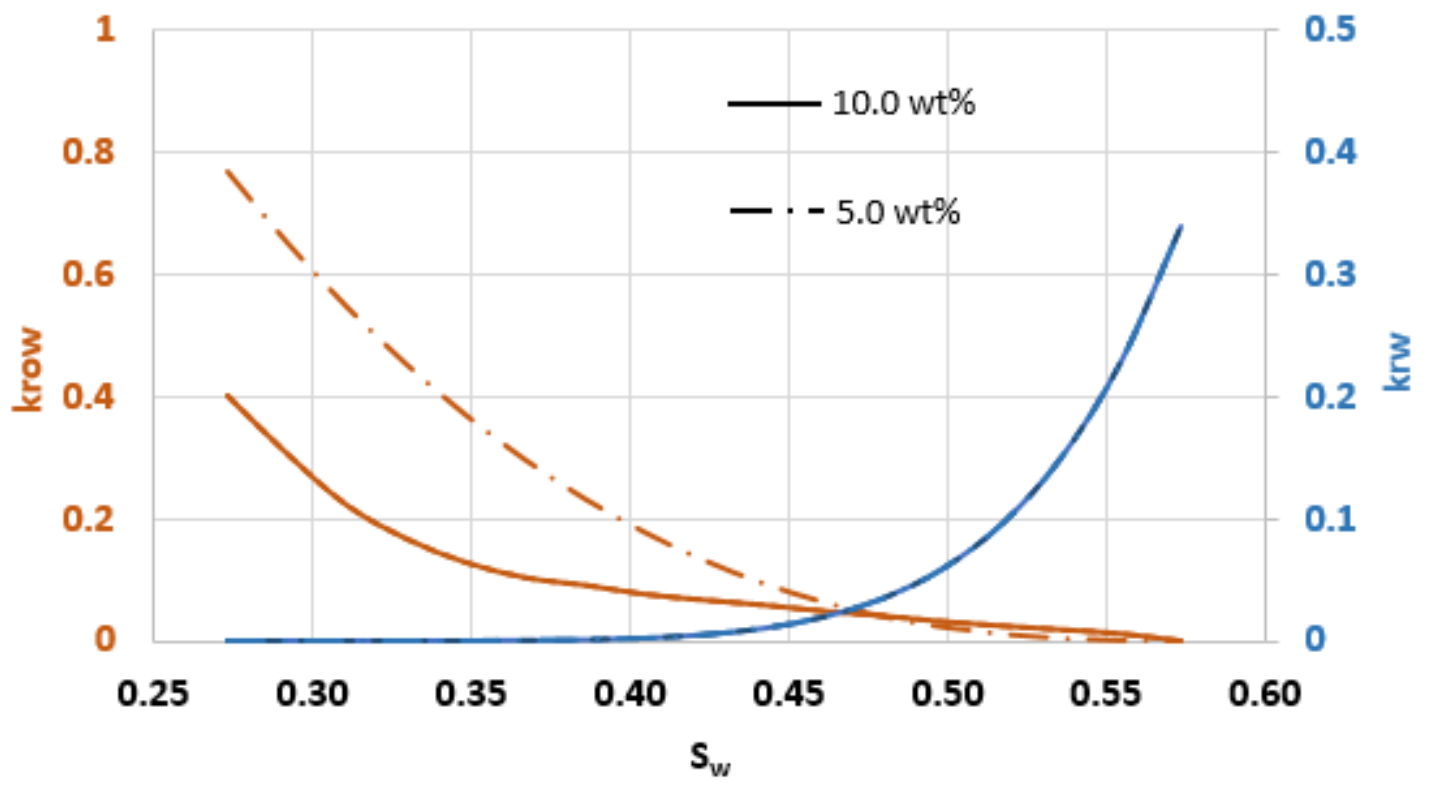

Figure 6.11 Steam plus $\mathrm{CO}_{2}-$ Oil-water relative permeability curves 


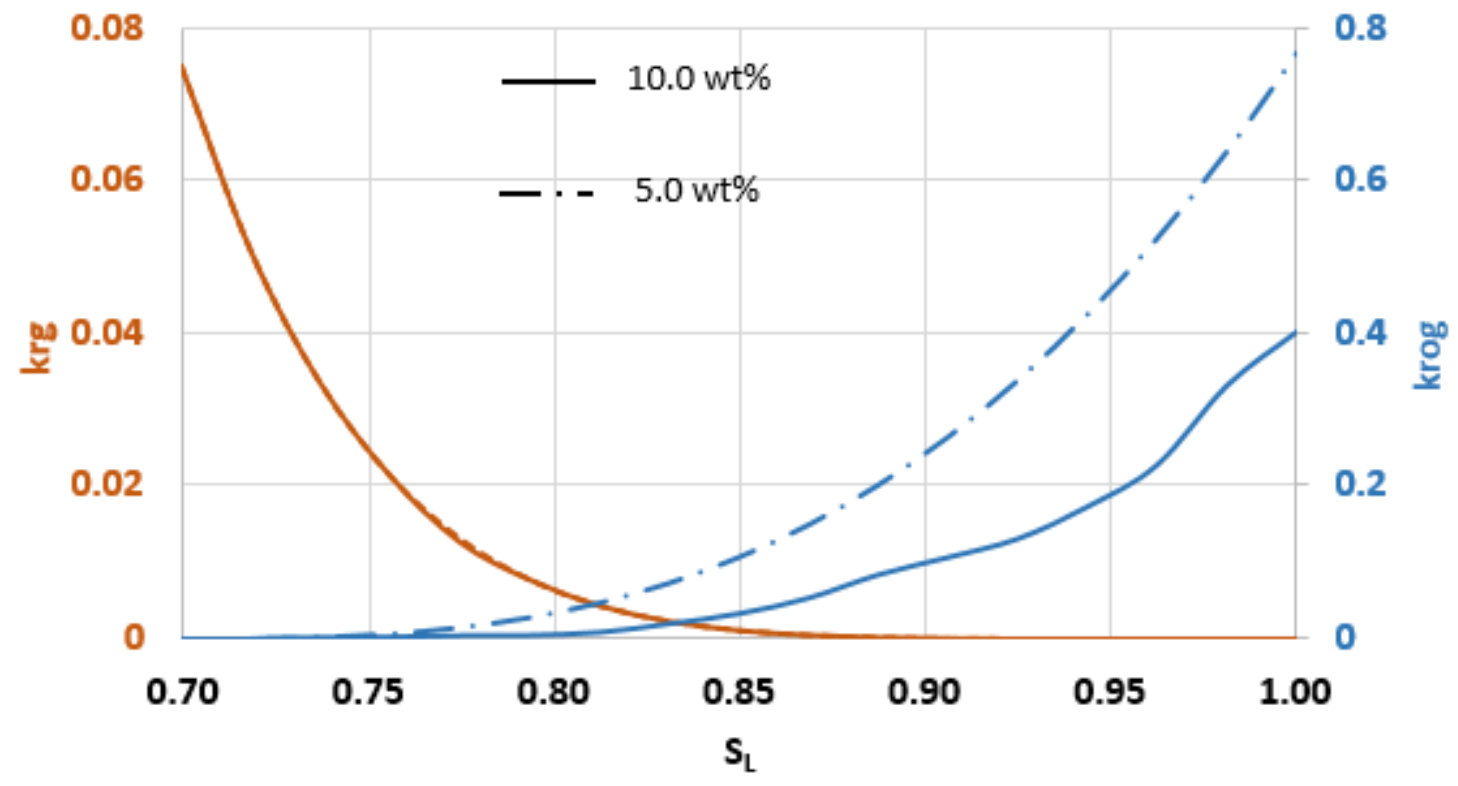

Figure 6.12 Steam plus $\mathrm{CO}_{2}-$ Oil-gas relative permeability curves

\subsection{History-Match Results for Steam plus Nitrogen Experiments}

As a starting point, to match the experimental results of the steam plus nitrogen experiments, the relative permeability curves for the $5.0 \mathrm{wt} \%$ carbon dioxide experiments were used. As well, all the required inputs, for example, the injection rate, injection composition, PVT model for nitrogen, injection and production pressure, and rate constraints were provided to the numerical model. The relative permeability curves were used as the history-matching parameter.

For each experiment, hundreds of simulations were run to match the injection and production data. The history-matched results with corresponding relative permeability curves are presented in this dissertation.

Figure 6.13 presents the history-matched results for produced oil for the $5.0 \mathrm{wt} \%$ nitrogen experiment. The initial production period was matched better than in the carbon dioxide test. However, this period was still slightly underpredicted by the simulation. After 3 hours, the production was closely matched. The relative error at the end of production was $0.7 \%$. 


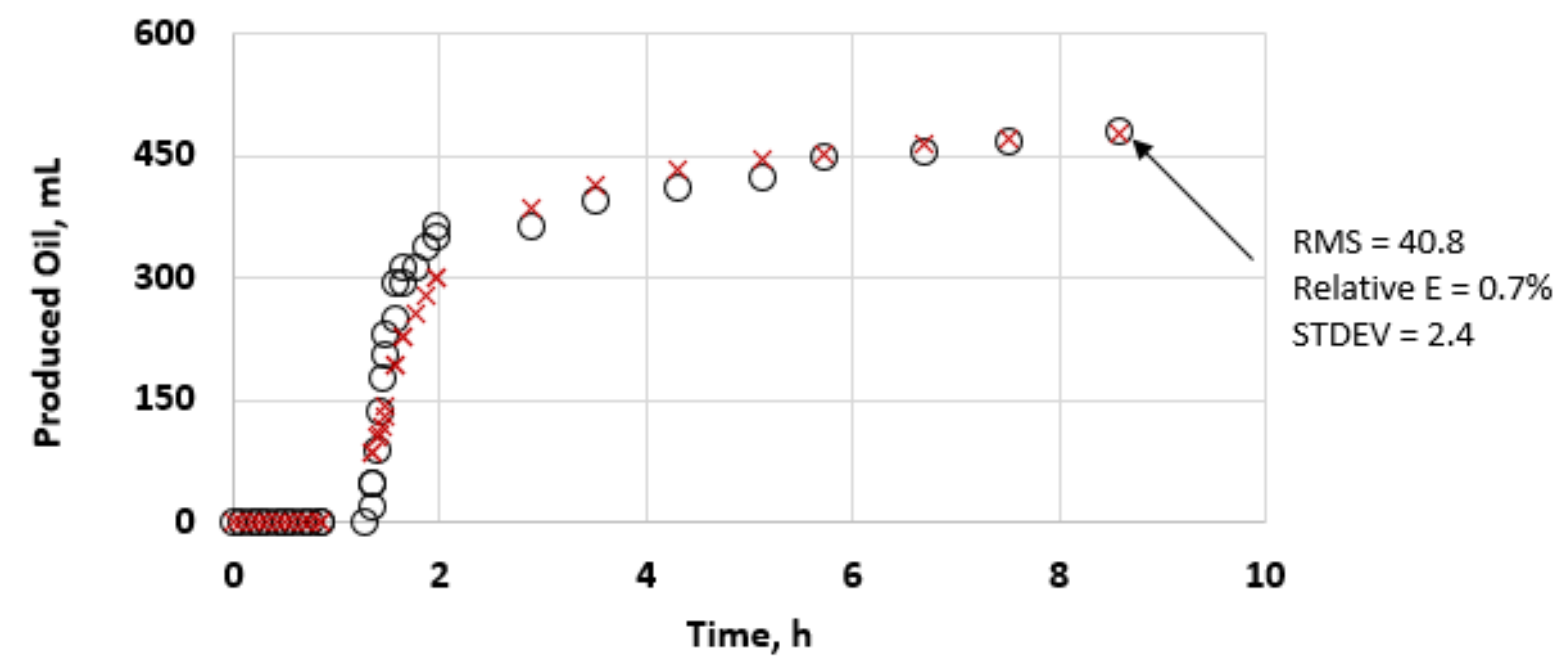

O Experimental

$\times$ Simulated

Figure 6.13 Steam plus $5.0 \mathrm{wt} \% \mathrm{~N}_{2}-$ Oil production history-match

Interestingly, the oil-water relative permeability curves for the $5.0 \mathrm{wt} \%$ carbon dioxide experiment did not need to be altered to provide this match. Although the solubility of nitrogen at experimental conditions was much less than that of carbon dioxide, even then the additional viscosity reduction from carbon dioxide did not seem sufficient to overtake the effect of enhanced oil recovery from additional moles of nitrogen injected. Note that at the same weight percentage, the mole percent of nitrogen is one and a half times that of carbon dioxide (i.e., about 3.0 mole percent of nitrogen compared to about 2.0 mole percent of carbon dioxide).

Figure 6.14 presents the experimental and simulated results for produced oil for the $7.5 \mathrm{wt} \%$ nitrogen experiment. The relative permeability curves were tuned to match the experimental data. Similarly to the $5.0 \mathrm{wt} \%$ simulation, the numerical model underpredicted the oil recovery during the initial injection times. After around 3 hours of injection, the predictions were slightly higher than the actual data; however, by the end of the production, the error between the experimental value and prediction was $2.1 \%$. 


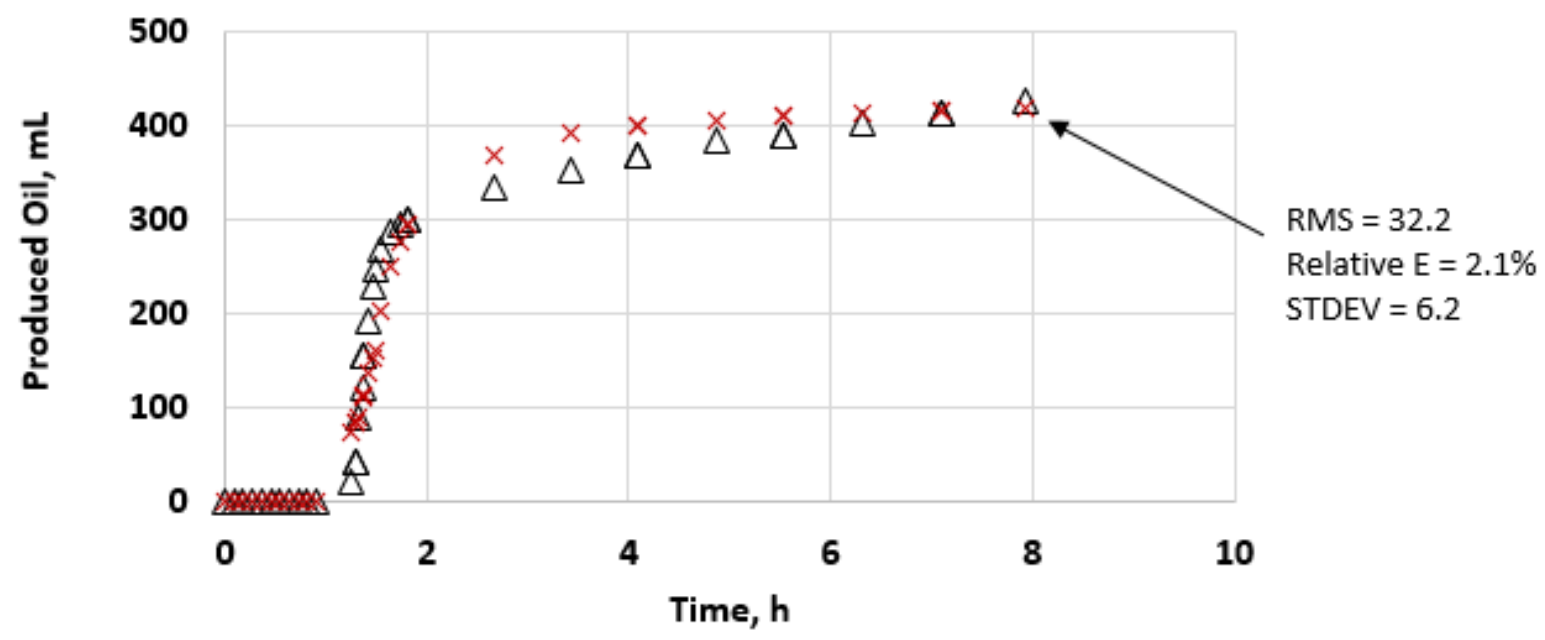

$\Delta$ Experimental

$\times$ Simulation

Figure 6.14 Steam plus $7.5 \mathrm{wt} \% \mathrm{~N}_{2}$ - Oil production history-match

Figure 6.15 presents the experimental and simulated results for produced oil for the $10.0 \mathrm{wt} \%$ nitrogen experiment. The relative permeability curves were tuned to match the experimental results. The initial production period up to 3 hours was slightly underpredicted; however, the match was much better compared to the previously presented history-matches. The experimental results were matched very closely, and the relative error by the end of the production period was $0.8 \%$.

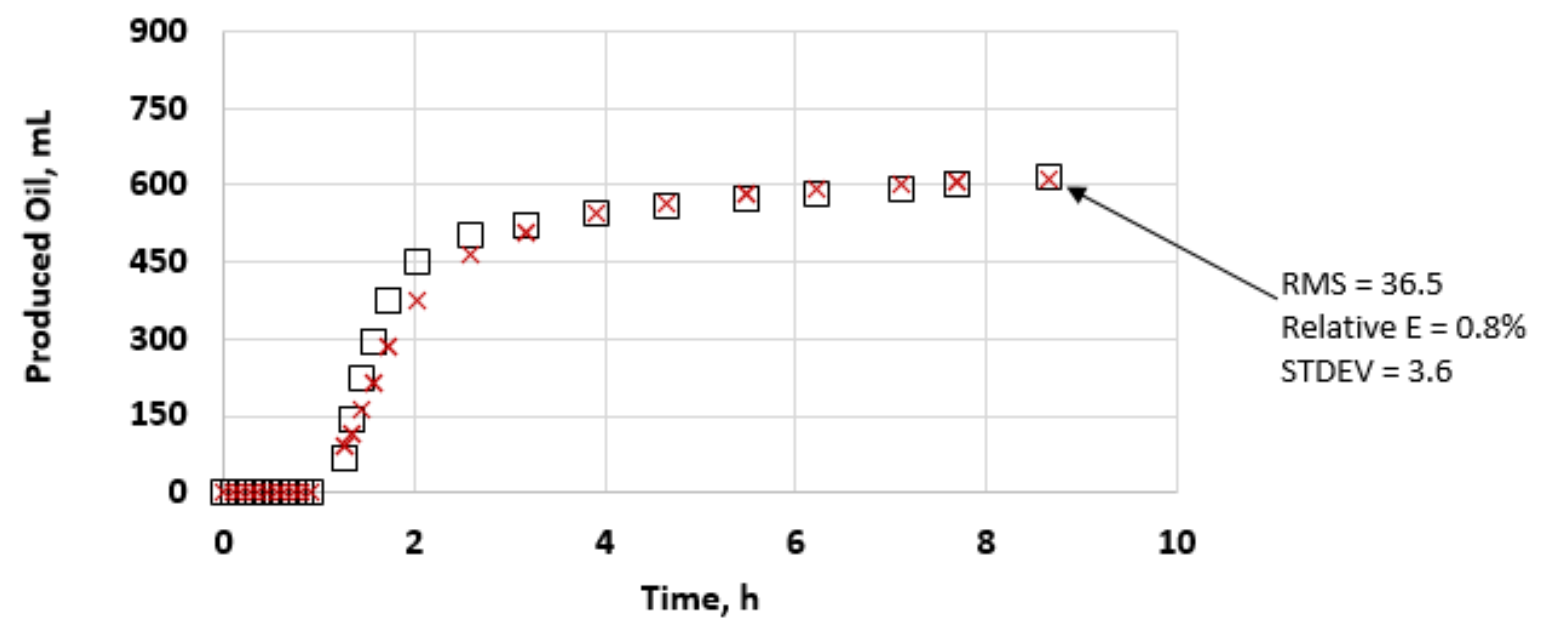

$\square$ Experimental

$\times$ Simulation

Figure 6.15 Steam plus $10.0 \mathrm{wt} \% \mathrm{~N}_{2}$ - Oil production history-match 
Figure 6.16 presents the experimental and simulated results for produced gas for the $5.0 \mathrm{wt} \%$ nitrogen experiment. The simulation model showed earlier production of gas. However, compared to the $5.0 \mathrm{wt} \%$ carbon dioxide experiment, the delay between the simulated start time of gas production and the actual start time was much improved during this simulation. The relative error by the end of the production period was $-3.4 \%$. Overall this history-match was even better than the match for the $5.0 \mathrm{wt} \%$ carbon dioxide experiment.

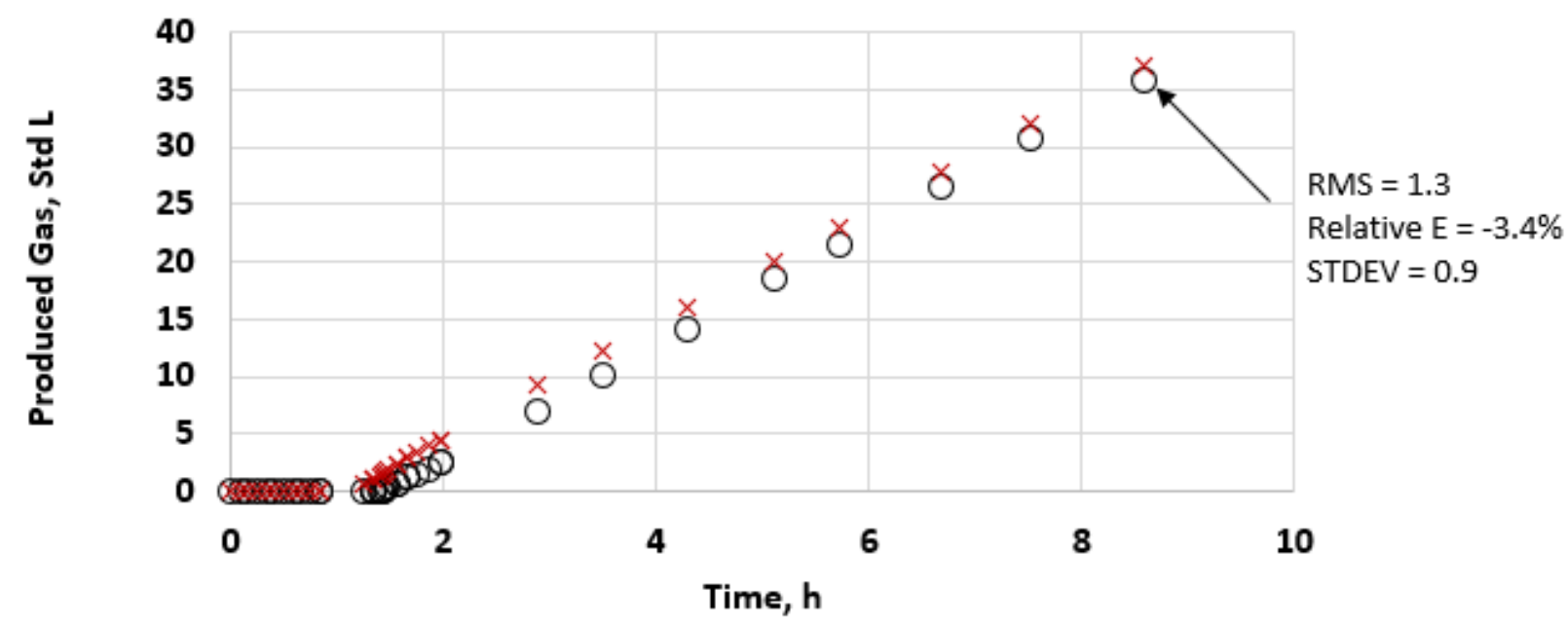

O Experimental

$\times$ Simulated

Figure 6.16 Steam plus $5.0 \mathrm{wt} \% \mathrm{~N}_{2}-$ Gas production history-match

Figure 6.17 presents the experimental and simulated results for produced gas for the $7.5 \mathrm{wt} \%$ nitrogen experiment. After two hours into production, the simulated results were closely predicted. By the end of the experiment, the relative error was $-5.8 \%$.

Figure 6.18 presents the experimental and simulated results of produced gas for $10.0 \mathrm{wt} \%$ nitrogen experiment. Overall a reasonable history-match was achieved. Similar to the other simulations, during the initial hours the simulated results were a bit off, due to earlier gas production, and later it started to improve. The simulation model slightly over predicted the produced gas. The relative error at the end of production was $-3.5 \%$. 

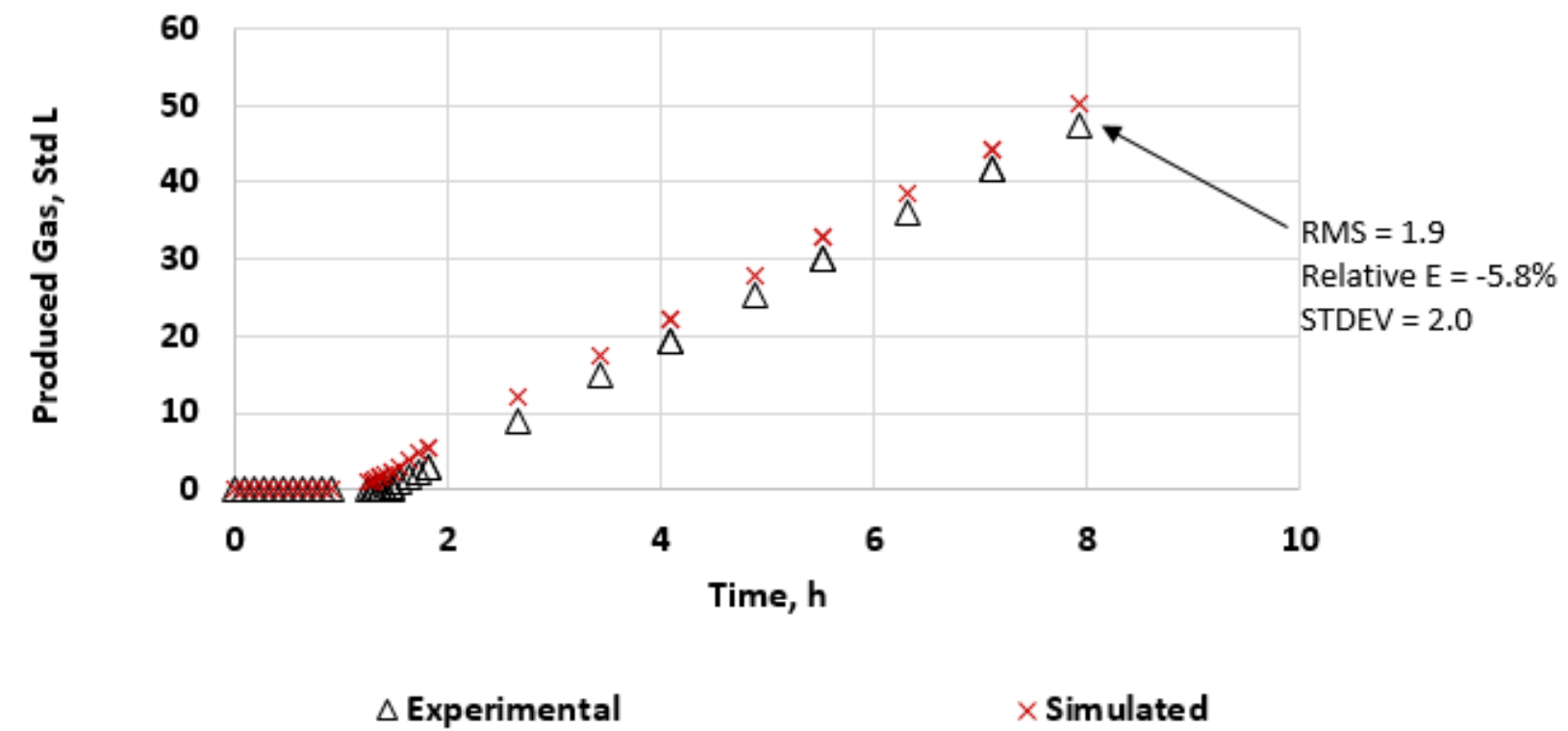

Figure 6.17 Steam plus $7.5 \mathrm{wt} \% \mathrm{~N}_{2}-$ Gas production history-match

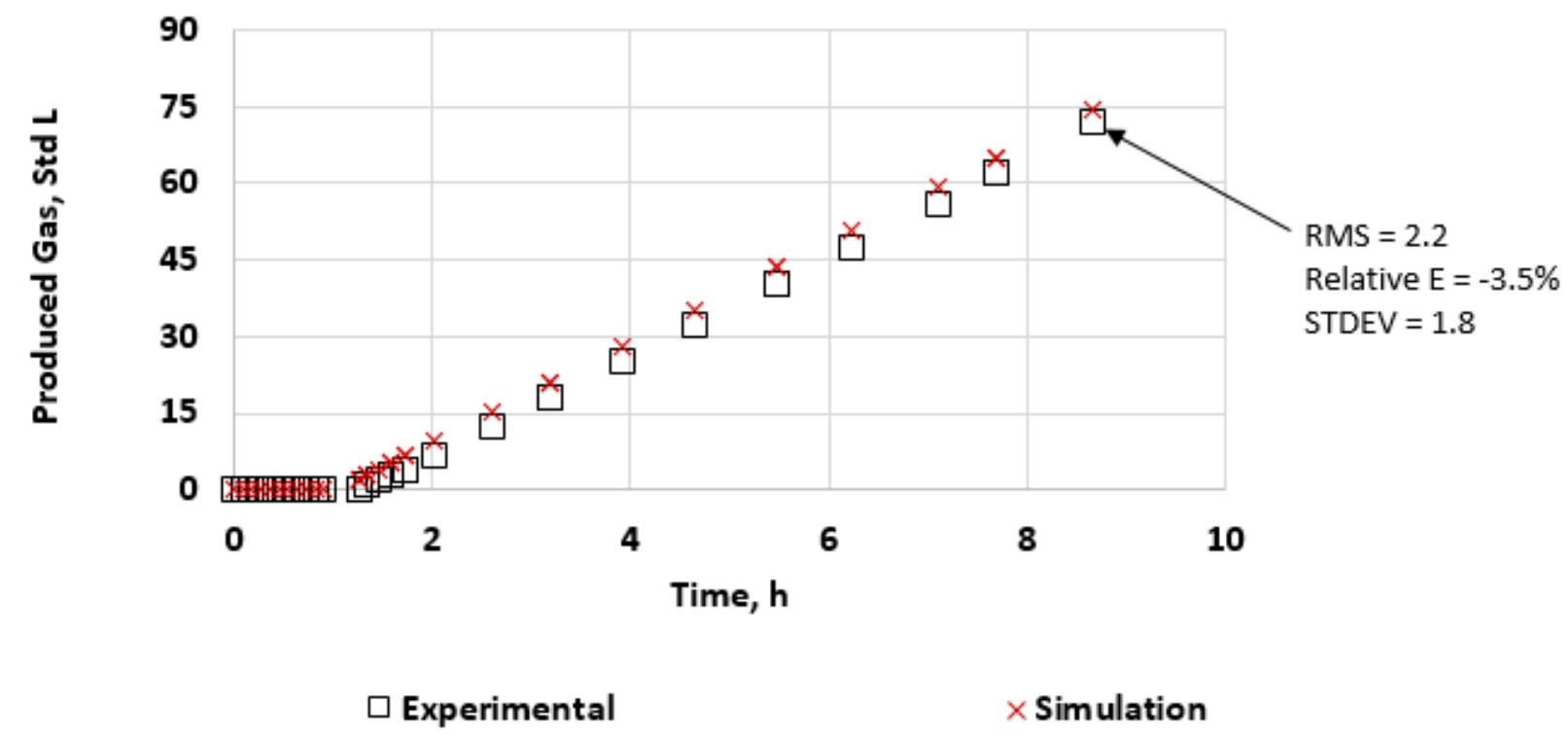

Figure 6.18 Steam plus $10.0 \mathrm{wt} \% \mathrm{~N}_{2}-$ Gas production history-match 
Figure 6.19 presents the experimental and history-matched results for produced water for the 5.0 wt $\%$ nitrogen experiment. As indicated in the previous produced water history-matches, the initial water production in the experiments was not that uniform. Probably this was an experimental artifact arising from the use of pumps as a production system. Overall, a good history-match was obtained. The relative error at the end of production was $-1.2 \%$.

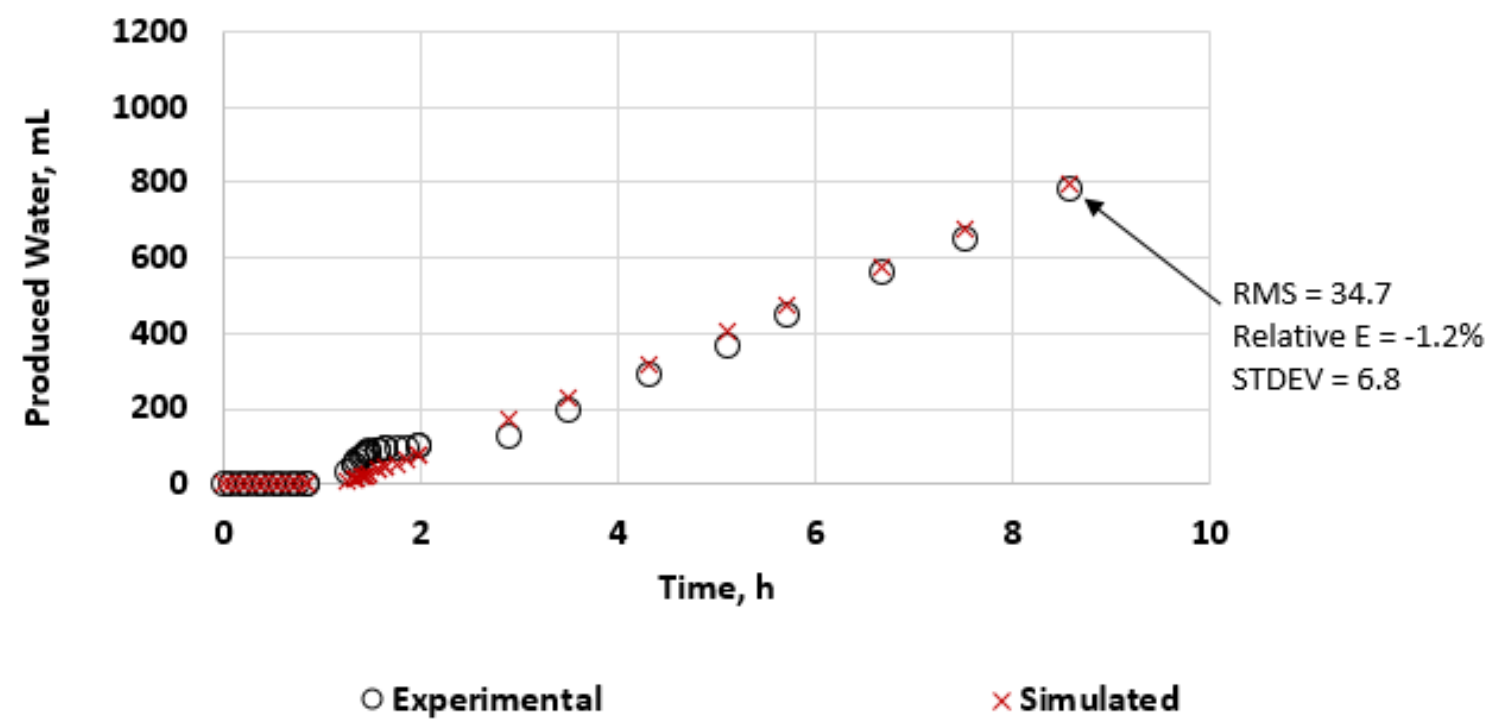

Figure 6.19 Steam plus $5.0 \mathrm{wt} \% \mathrm{~N}_{2}$ - Water production history-match

Figure 6.20 presents the experimental and predicted results for produced water for the $7.5 \mathrm{wt} \%$ nitrogen experiment. Apart from the initial production period (before two hours), the simulation model predicted the produced water well. The error at the end of production was $2.7 \%$.

Figure 6.21 presents the experimental and history-matched results for produced water for the 10.0 wt $\%$ nitrogen experiment. The history-match was slightly off in two instances. The first instance is at the early times, and the second is around seven hours into production. The experimental results were non-uniform at both times. Again, this can be attributed to a slight error in the measurements. Other than that, the simulation model history-matched the experimental data very well. The relative error at the end of production was $-2.0 \%$. 


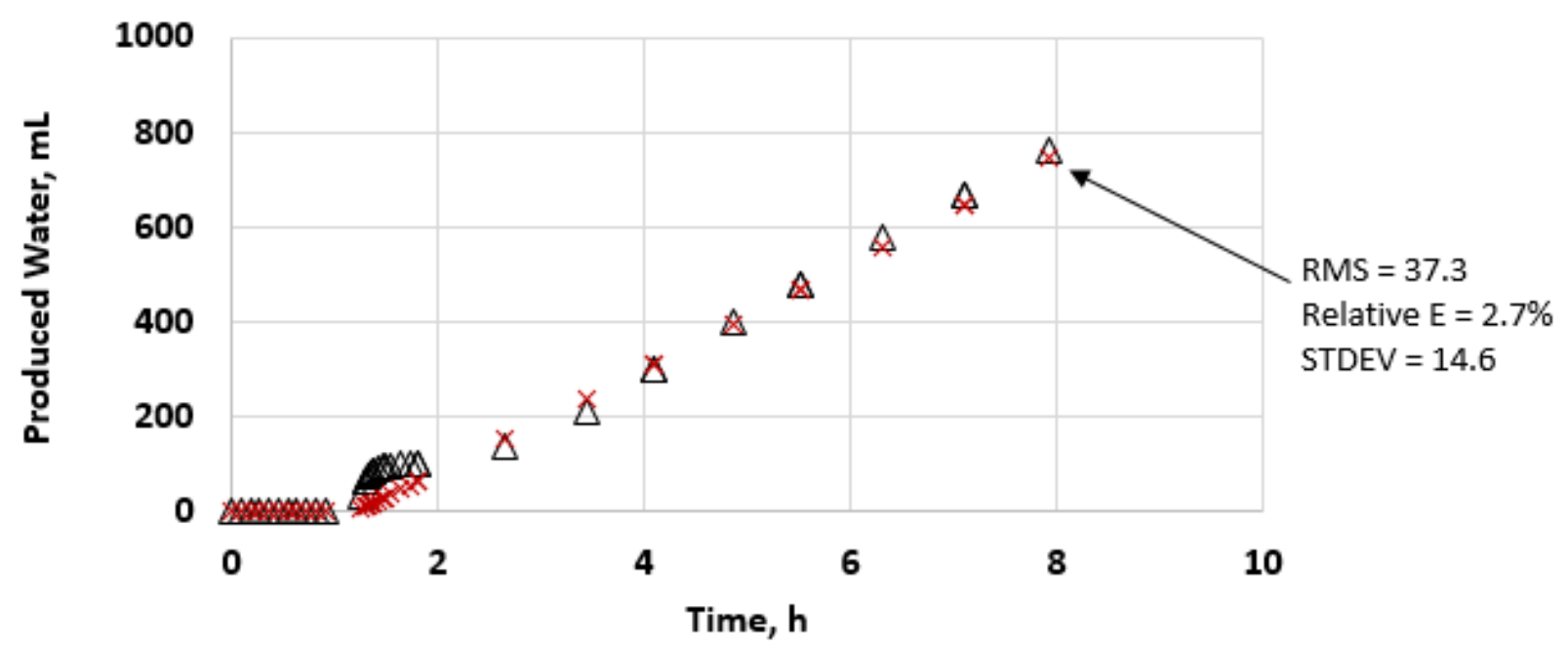

$\Delta$ Experimental

$\times$ Simulated

Figure 6.20 Steam plus $7.5 \mathrm{wt} \% \mathrm{~N}_{2}$ - Water production history-match

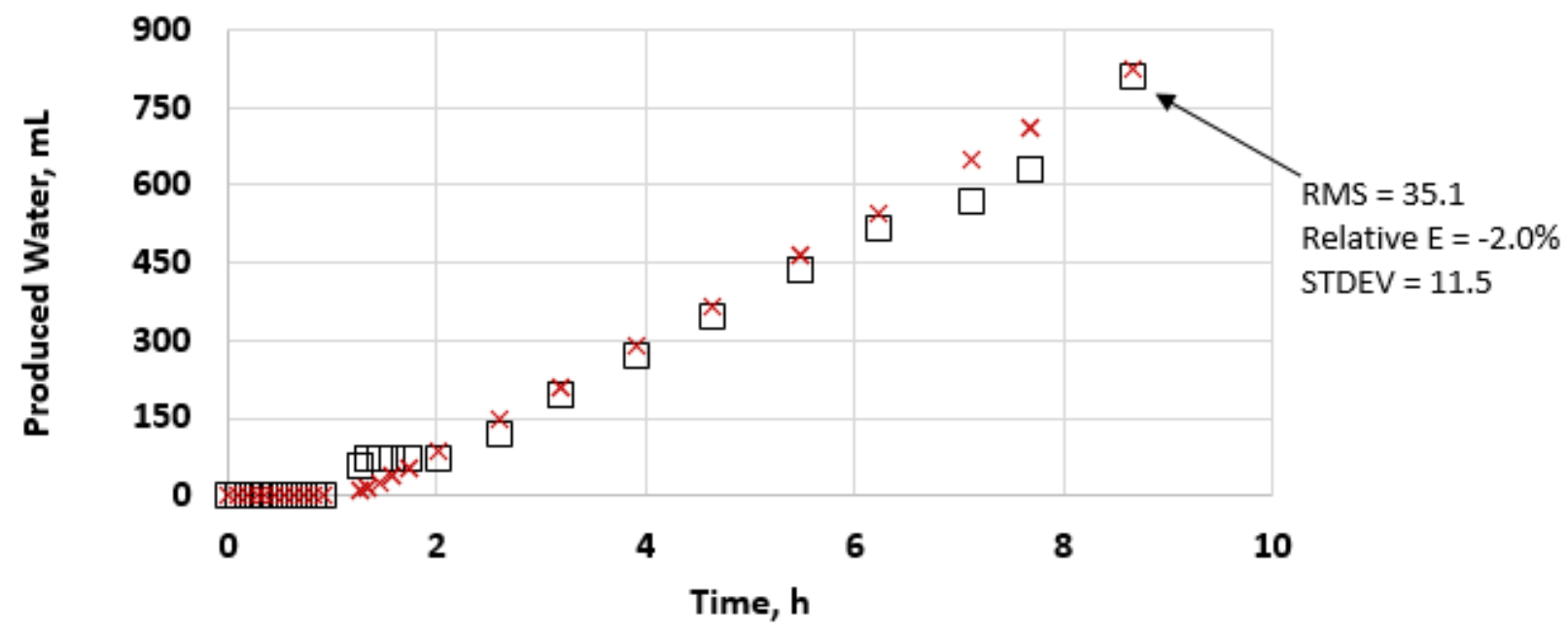

$\square$ Experimental

$\times$ Simulation

Figure 6.21 Steam plus $10.0 \mathrm{wt} \% \mathrm{~N}_{2}-$ Water production history-match

Figure 6.22 presents the tuned relative permeability curves for oil and water. Figure 6.23 presents the same for oil and gas. Note that oil-water relative permeability curves for the $5.0 \mathrm{wt} \%$ nitrogen experiment are the same as for the $5.0 \mathrm{wt} \%$ carbon dioxide experiment. The circles correspond to 
the $7.5 \mathrm{wt} \%$ experiment, the dotted line to the $5.0 \mathrm{wt} \%$ experiment, and the solid lines to the 10.0 wt\% experiment.

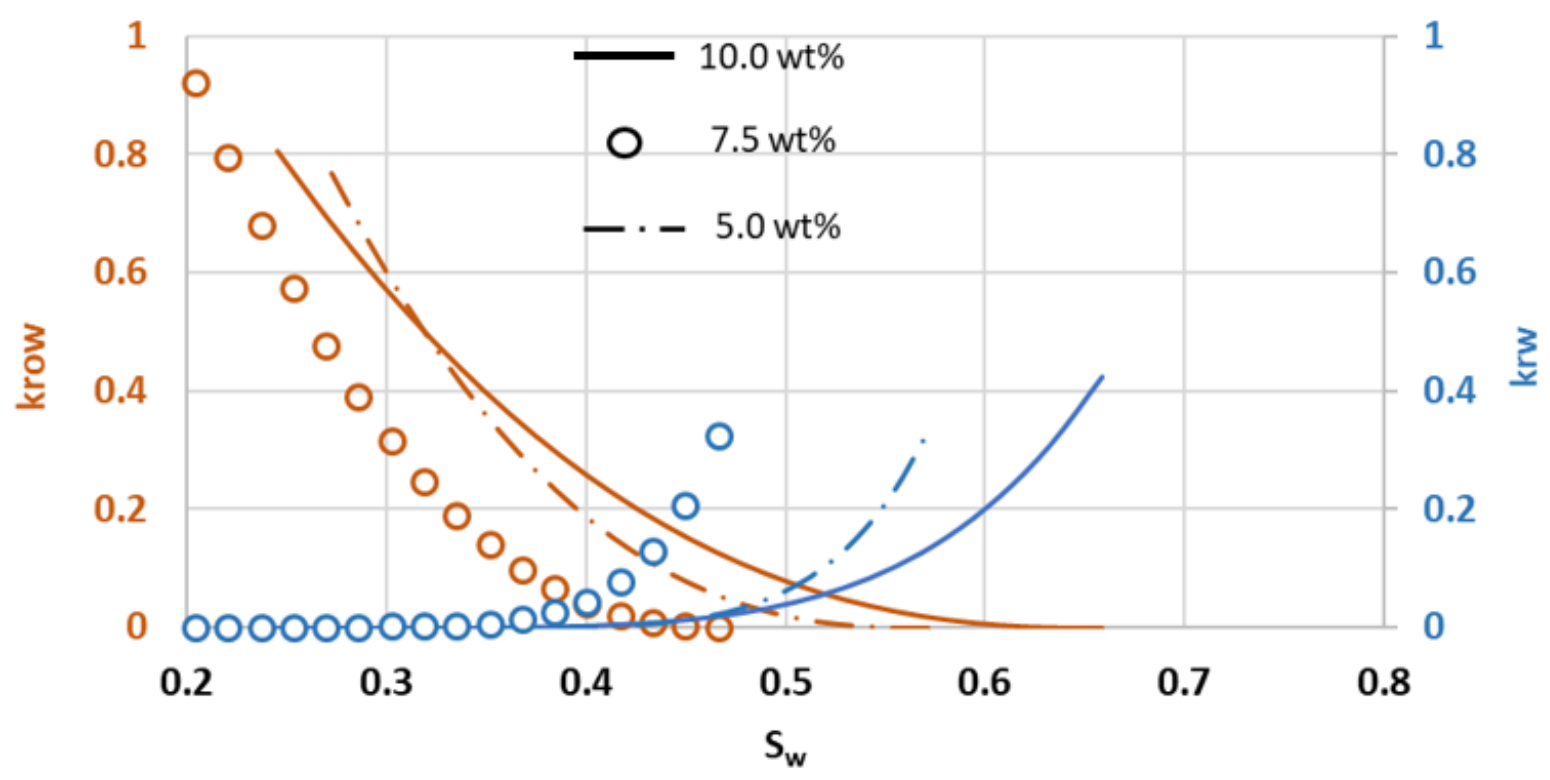

Figure 6.22 Steam plus $\mathrm{N}_{2}-$ Oil-water relative permeability curves

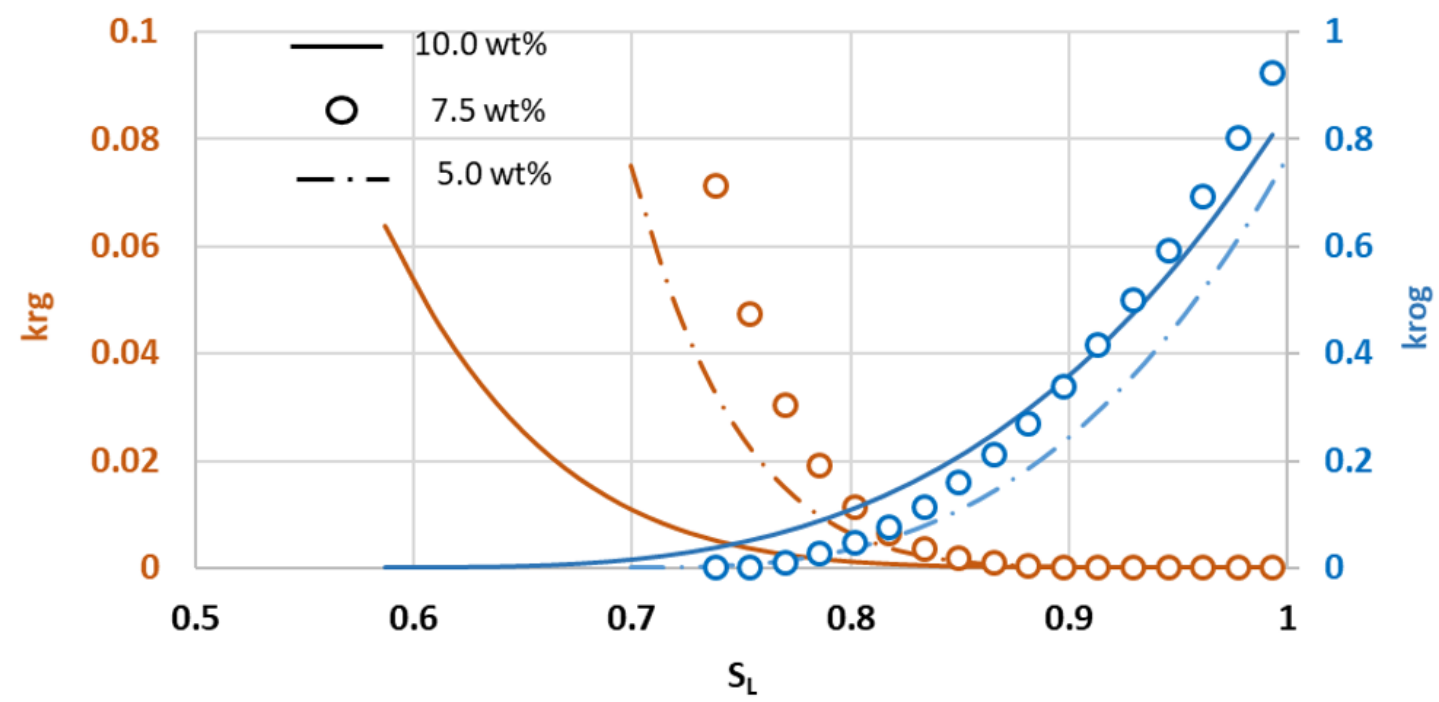

Figure 6.23 Steam plus $\mathrm{N}_{2}-$ Oil-gas relative permeability curves 


\subsection{History-Match Results for Steam plus Methane Experiments}

As a starting point to match the experimental results for steam plus methane experiments, the relative permeability curves for the $5.0 \mathrm{wt} \%$ nitrogen experiments were used; as well, all the required inputs, for example, the injection rate, injection composition, PVT model for methane, injection and production pressure, and rate constraints were provided to the numerical models. The relative permeability curves for both the oil and water and oil and gas were altered to obtain a better history-match.

For each experiment, hundreds of simulations were run to match the injection and production data. The history-matched results with corresponding relative permeability curves are presented in this dissertation.

Figure 6.24 presents the experimental and simulated results for produced oil for the $5.0 \mathrm{wt} \%$ methane experiment. Compared to the carbon dioxide and nitrogen experiments, the initial production period was matched better. The initial production period was slightly over predicted by simulations. After 2 hours, the production was closely matched. The relative error at the end of production was $9.4 \%$.

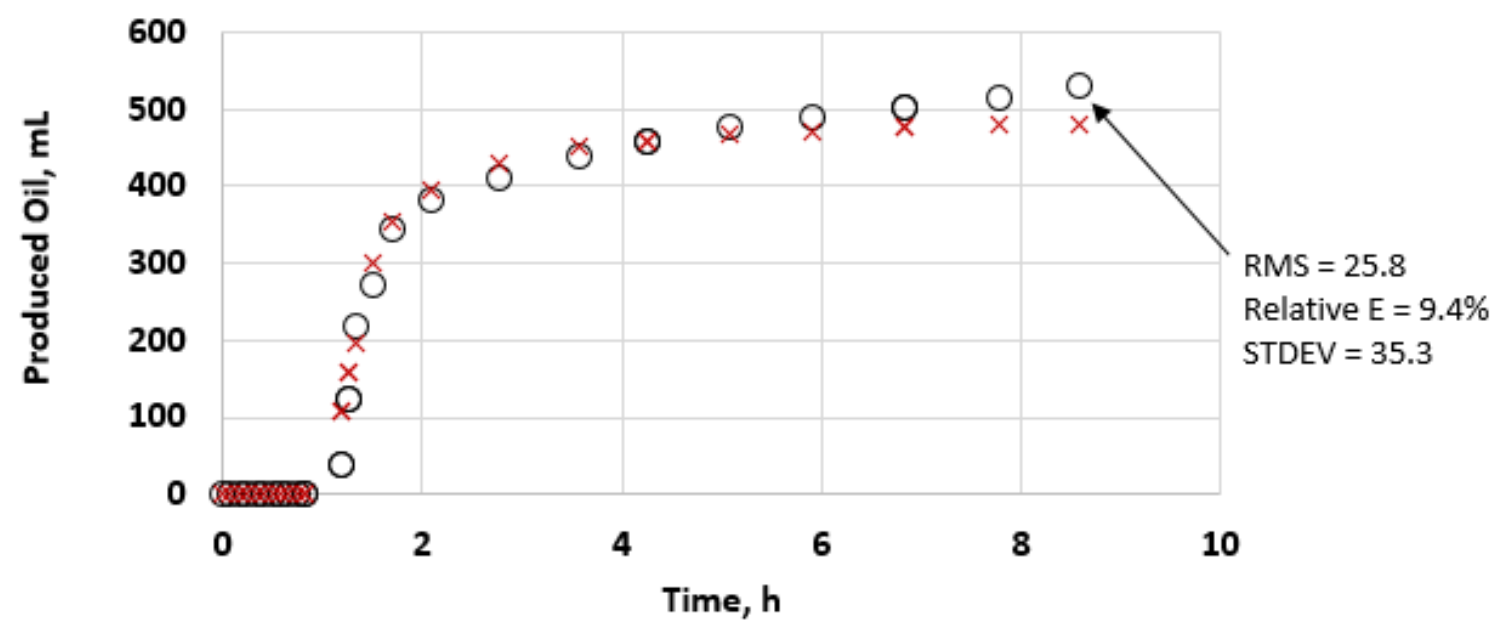

O Experimental

$\times$ Simulated

Figure 6.24 Steam plus $5.0 \mathrm{wt} \% \mathrm{CH}_{4}$ - Oil production history-match 
Figure 6.25 presents the experimental and simulated results for produced oil for the $7.5 \mathrm{wt} \%$ methane experiment. The relative permeability curves were tuned to match the experimental data. The numerical model predicted the experimental results very closely. At only one data point during the initial production period was the match a bit off. Later, for all the data points, the numerical model predictions were very close to the actual data. The relative error at the end of production was $-2.1 \%$.

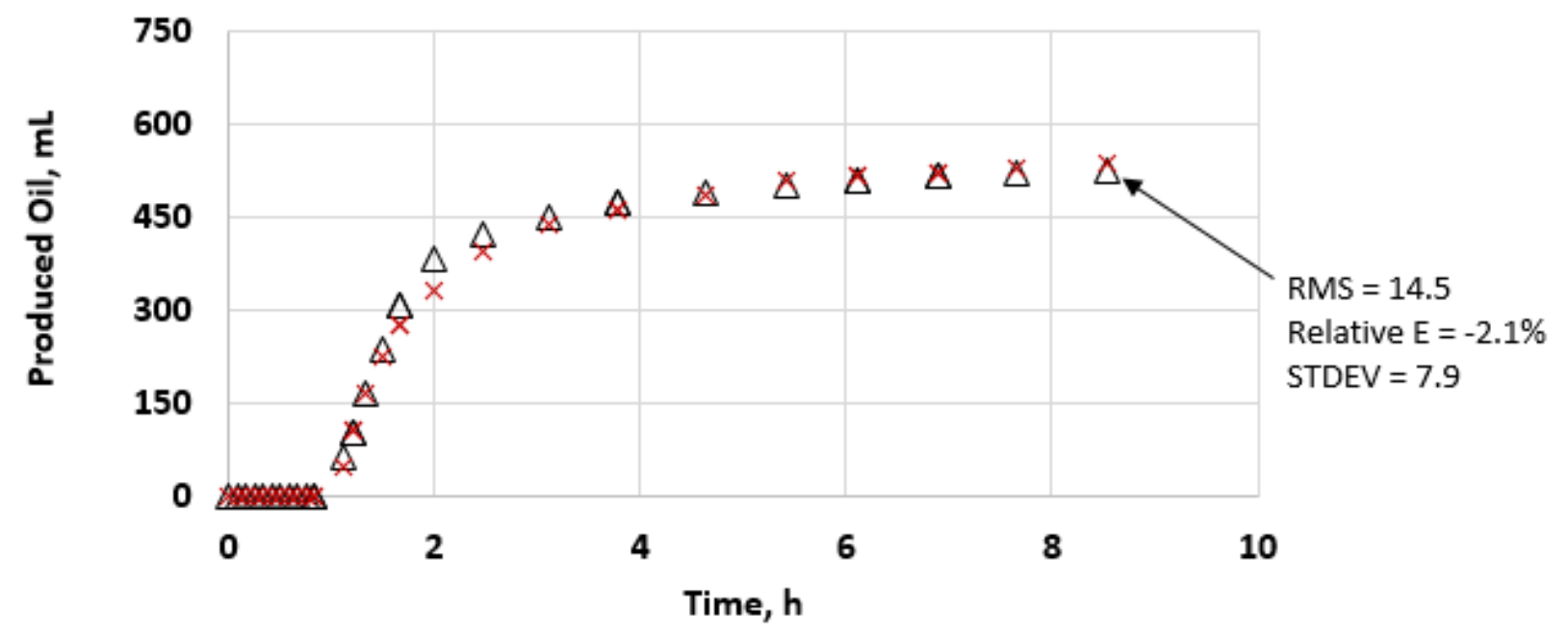

$\Delta$ Experimental

$\times$ Simulation

Figure 6.25 Steam plus $7.5 \mathrm{wt} \% \mathrm{CH}_{4}$ - Oil production history-match

Figure 6.26 presents the experimental and simulated results for produced oil for the $10.0 \mathrm{wt} \%$ methane experiment. The relative permeability curves were tuned to match the experimental results. The initial production period up to 3 hours was slightly overpredicted; however, a reasonably good history-match was obtained. The relative error at the end of production was $1.6 \%$.

Figure 6.27 presents the experimental and simulated results for produced gas for the $5.0 \mathrm{wt} \%$ methane experiment. Overall a reasonable history-match was achieved. The relative error at the end of production was $-5.4 \%$. The simulation slightly overpredicted the produced gas. 


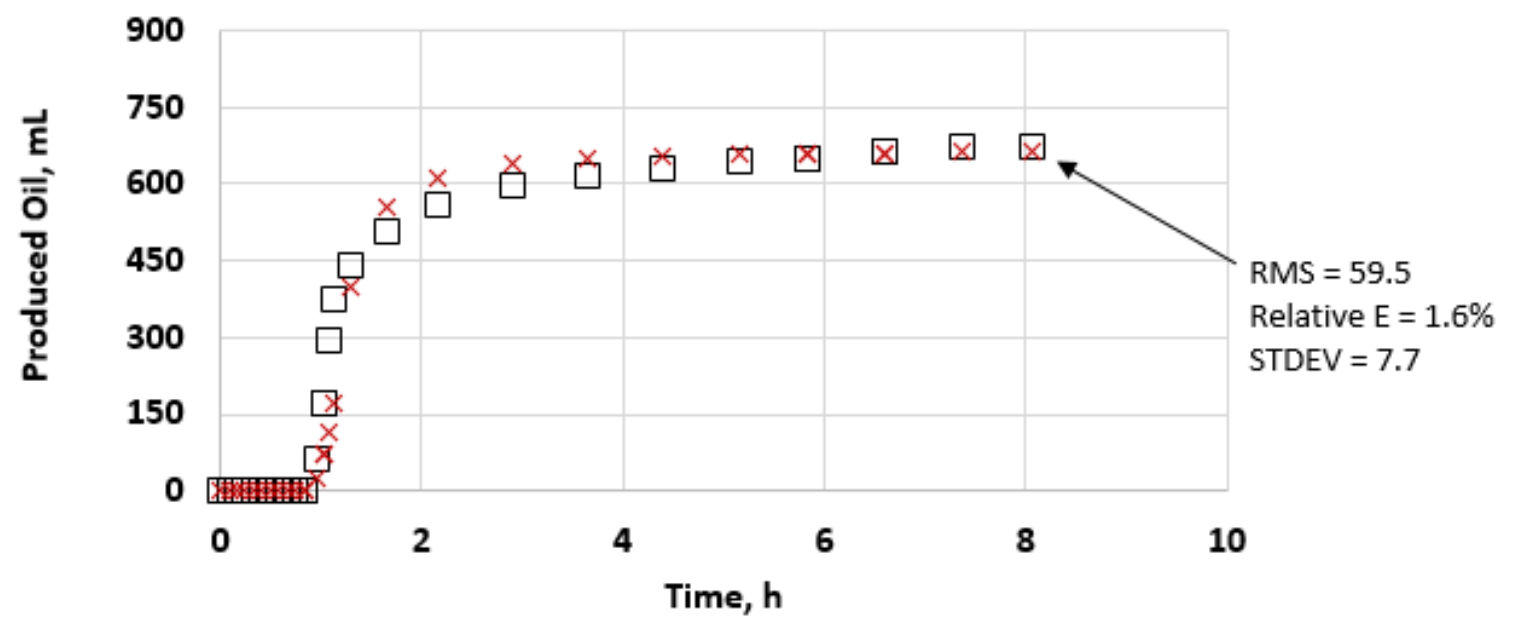

Experimental

$\times$ Simulation

Figure 6.26 Steam plus $10.0 \mathrm{wt} \% \mathrm{CH}_{4}$ - Oil production history-match

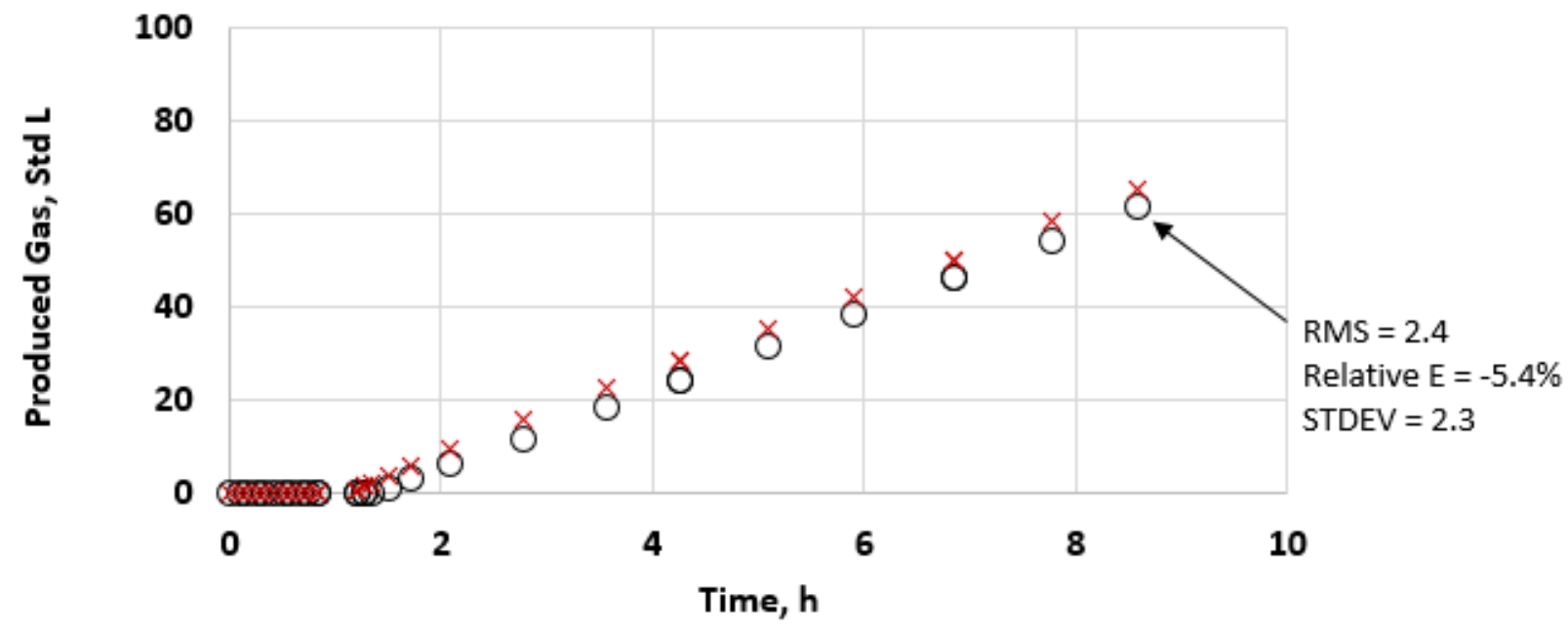

O Experimental

$\times$ Simulated

Figure 6.27 Steam plus $5.0 \mathrm{wt} \% \mathrm{CH}_{4}-$ Gas production history-match

Figure 6.28 presents the experimental and simulated results for produced gas for the $7.5 \mathrm{wt} \%$ methane experiment. By the end of production, the simulation predicted the cumulative produced gas with a relative error of $-1.7 \%$. 


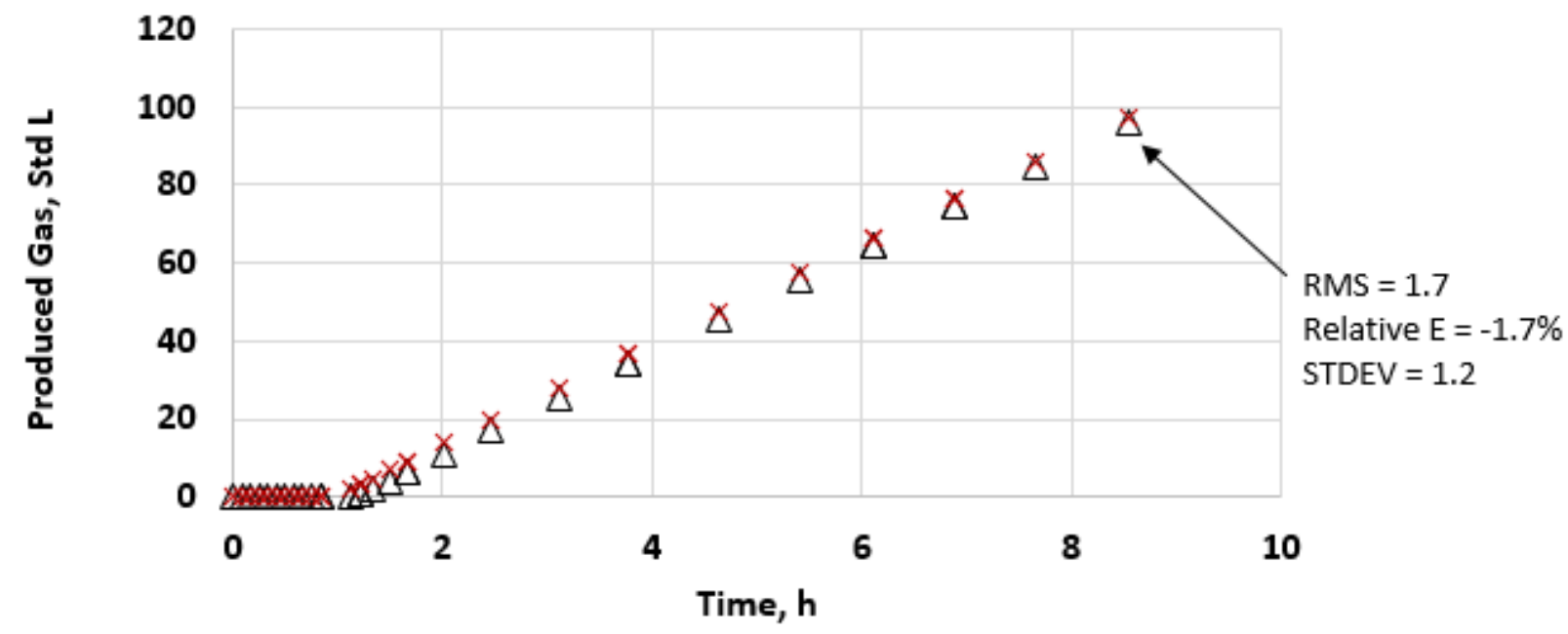

$\Delta$ Experimental $\quad \times$ Simulated

Figure 6.28 Steam plus $7.5 \mathrm{wt} \% \mathrm{CH}_{4}-$ Gas production history-match

Figure 6.29 presents the experimental and simulated results for produced gas for the $10.0 \mathrm{wt} \%$ methane experiment. Overall a reasonable history-match was achieved. The relative error at the end of production was $-4.7 \%$. The simulation slightly overpredicted the produced gas.

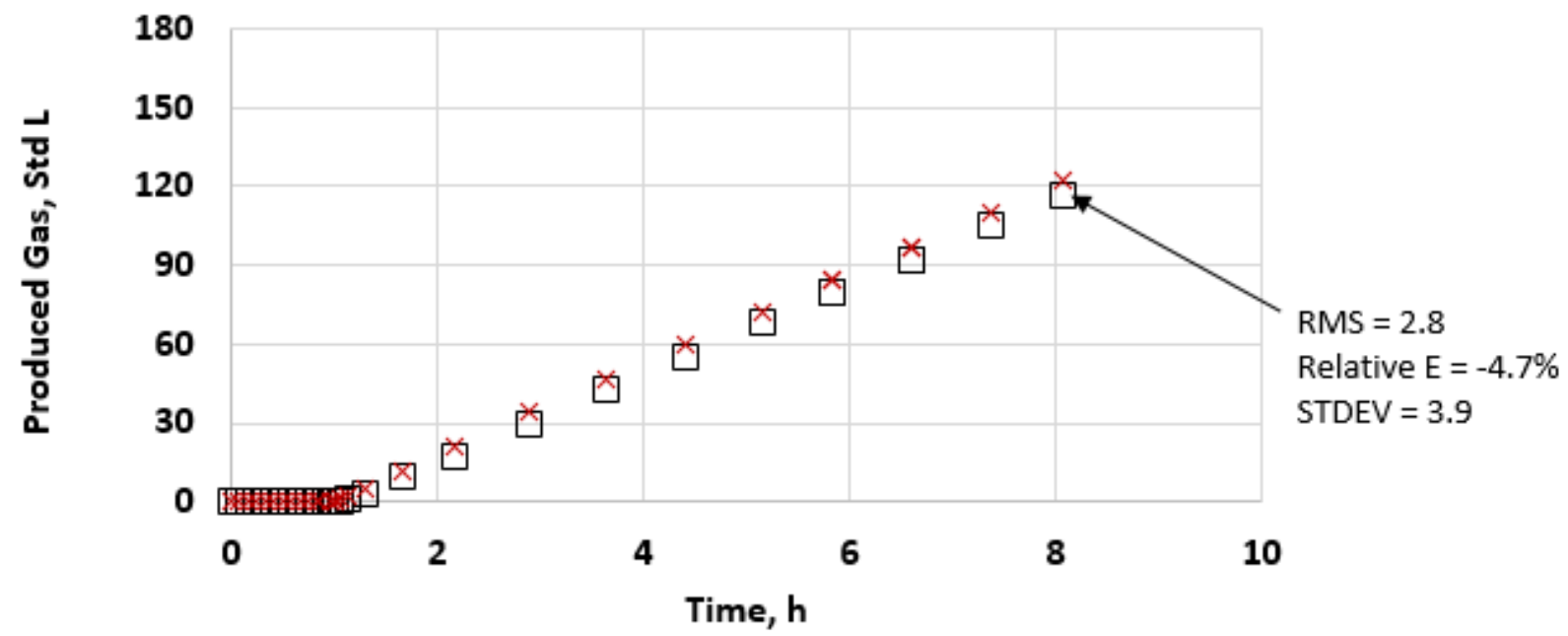

Experimental

$\times$ Simulated

Figure 6.29 Steam plus $10.0 \mathrm{wt} \% \mathrm{CH}_{4}-$ Gas production history-match 
Figure 6.30 presents the experimental and simulated results for produced water for the $5.0 \mathrm{wt} \%$ methane experiment. As indicated in the previous produced water history-matches, the initial water production in the experiments was not that uniform. Overall, a good history-match was obtained. The relative error at the end of production was $6.4 \%$.

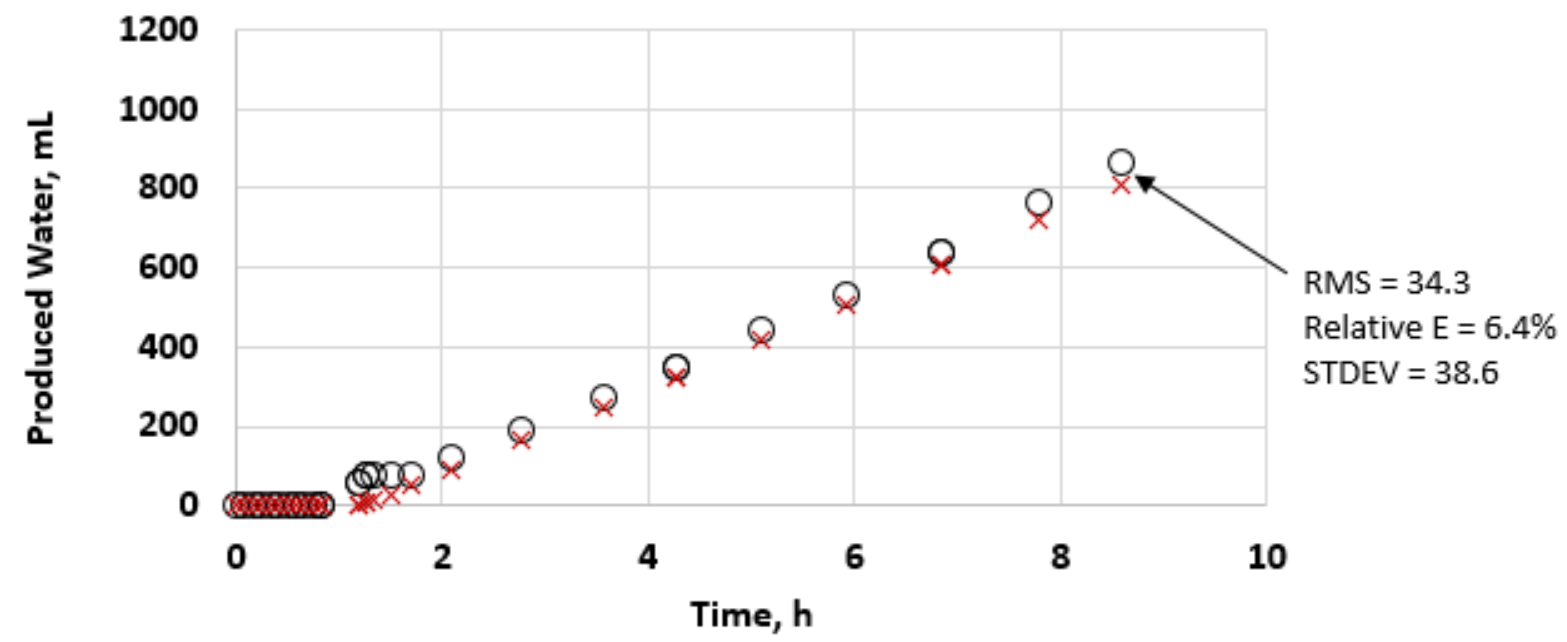

O Experimental

$\times$ Simulated

Figure 6.30 Steam plus $5.0 \mathrm{wt} \% \mathrm{CH}_{4}-$ Water production history-match

Figure 6.31 presents the experimental and simulated results for produced water for the $7.5 \mathrm{wt} \%$ methane experiment. Apart from the initial production period (before two hours), the simulation predicted the produced water very well. The error at the end of production was $1.4 \%$.

Figure 6.32 presents the experimental and simulated results for produced water for the $10.0 \mathrm{wt} \%$ methane experiment. The simulation history-matched the experimental data reasonably well. The relative error at the end of production was $2.5 \%$.. 


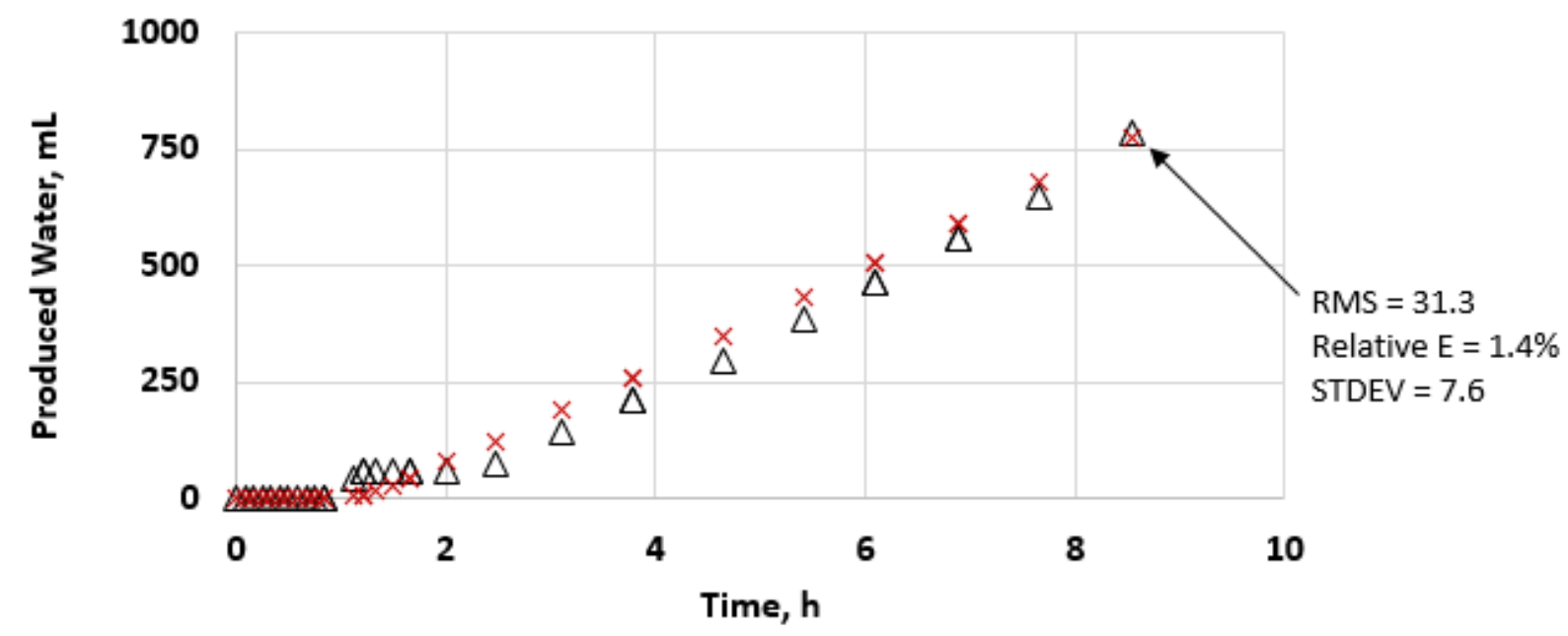

$\Delta$ Experimental

$\times$ Simulated

Figure 6.31 Steam plus $7.5 \mathrm{wt} \% \mathrm{CH}_{4}-$ Water production history-match

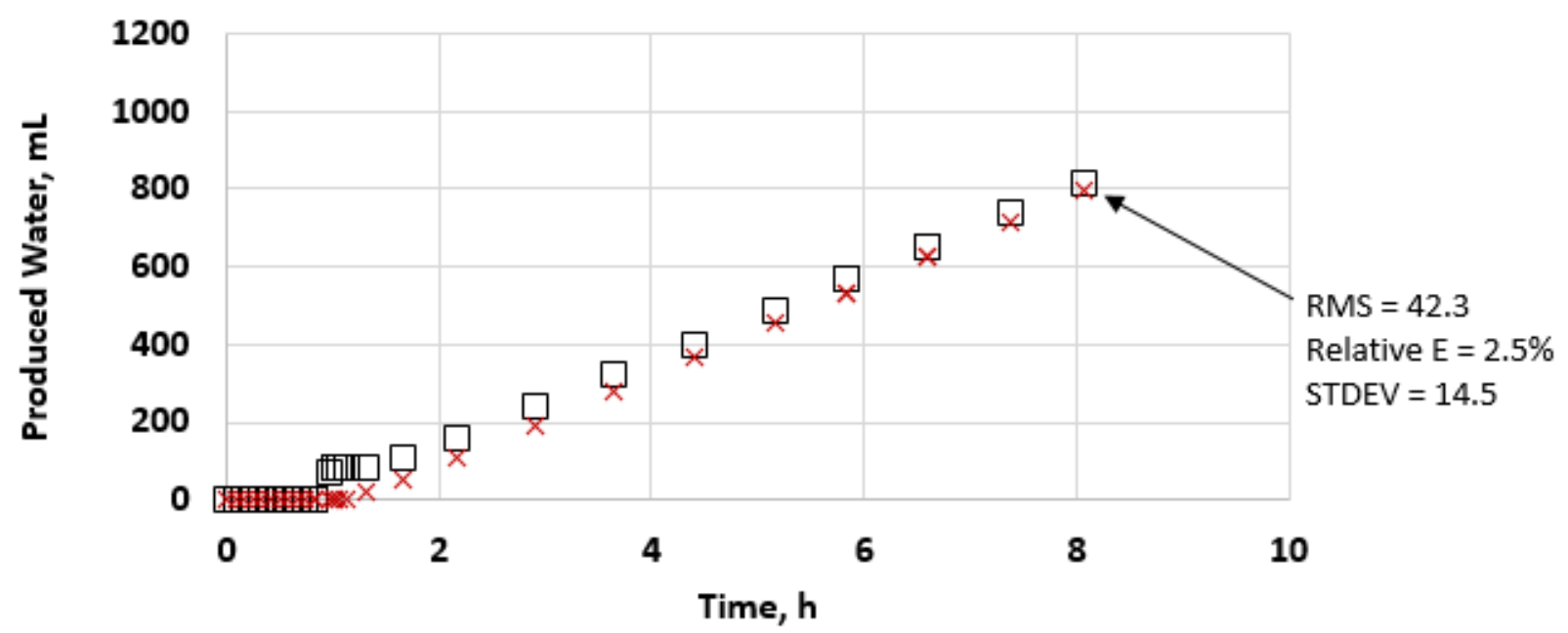

Experimental

$\times$ Simulated

Figure 6.32 Steam plus $10.0 \mathrm{wt} \% \mathrm{CH}_{4}-$ Water production history-match

For the experiments where methane was used as solvent, Figure 6.33 presents the tuned relative permeability curves for oil and water. Figure 6.34 presents the same for oil and gas. The circles correspond to the $7.5 \mathrm{wt} \%$ experiment, the dotted line to the $5.0 \mathrm{wt} \%$ experiment, and the solid lines to the $10.0 \mathrm{wt} \%$ experiment. 


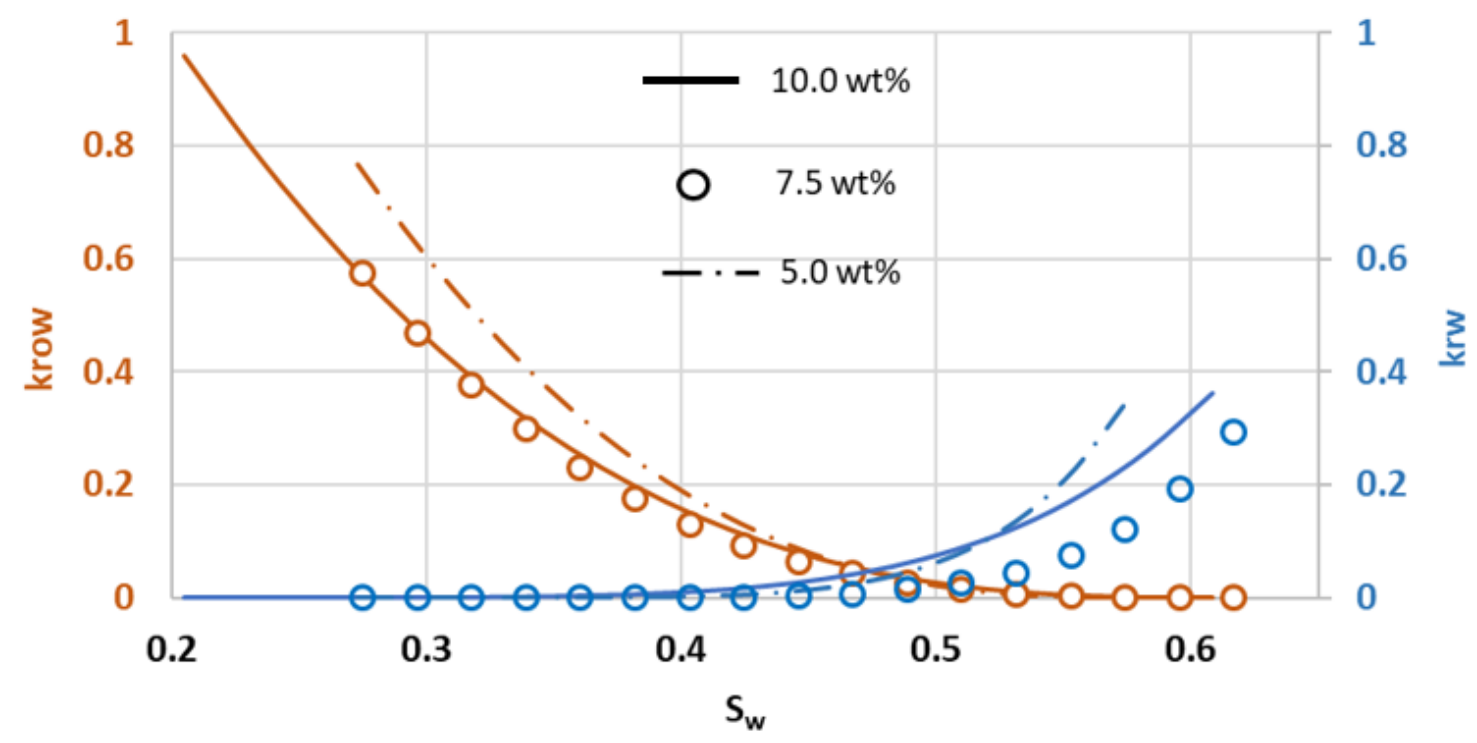

Figure 6.33 Steam plus $\mathrm{CH}_{4}-$ Oil-water relative permeability curves

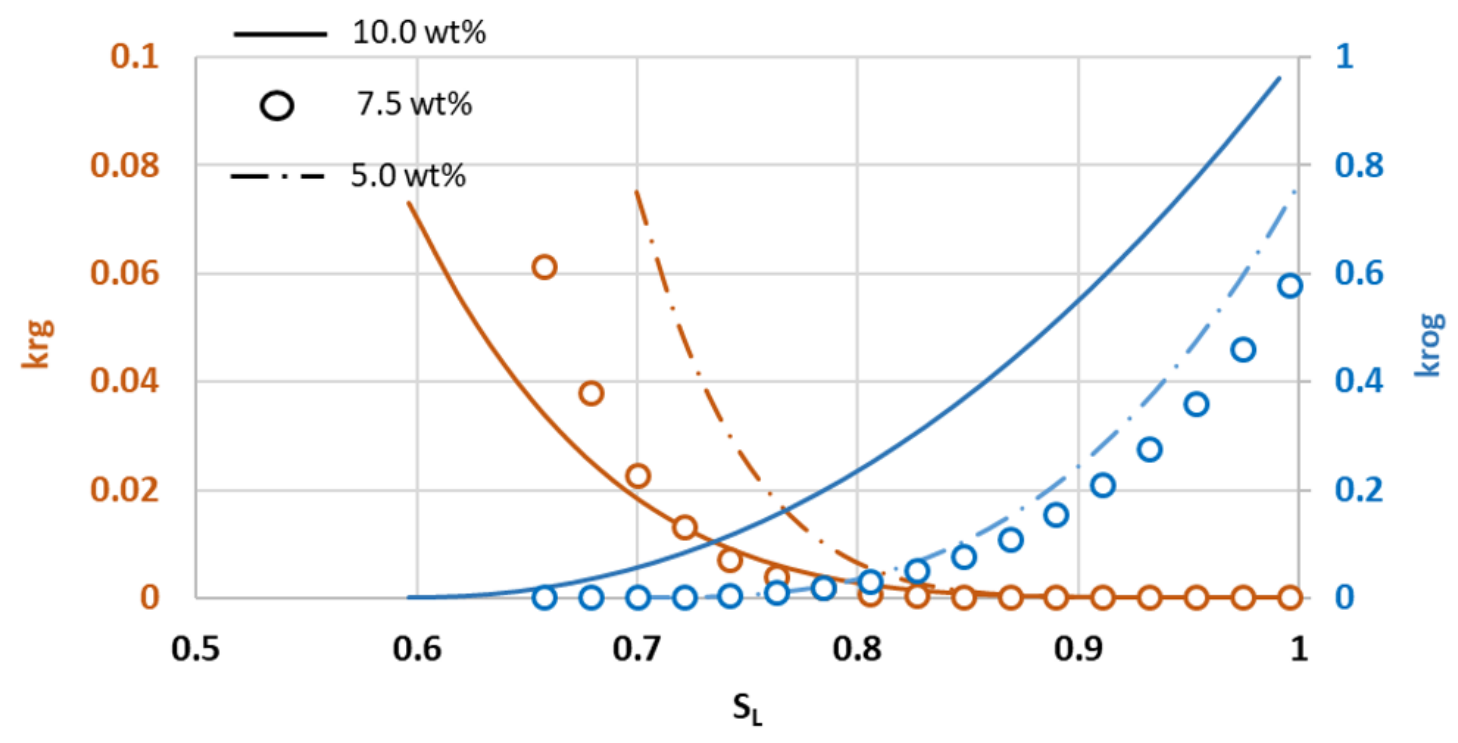

Figure 6.34 Steam plus $\mathrm{CH}_{4}$ - Oil-gas relative permeability curves 


\subsection{Summary of Important Observations and Findings}

The following are important observations and findings from the simulation study:

$\checkmark$ A clear trend was observed with respect to improvement in the predictions during the early periods for the gases with lower molecular weight. For example, for methane, which is the lightest gas among all those used during this study, the initial startup production match was better than for nitrogen and carbon dioxide.

$\checkmark$ The history-matches were within a reasonable relative error of up to $5.0 \%$.

$\checkmark$ The calibrated model can be said to describe the reservoir in an acceptable manner, and can be used for performance predictions.

$\checkmark$ The numerical model did not capture the early production period for any of the produced fluids. During the first three hours of production, the simulated data slightly overpredicted the produced fluids. 


\section{Conclusions and \\ Recommendations}

\subsection{Conclusions}

In this work, the performance of hybrid steam-assisted gravity drainage (SAGD) was evaluated for low pressure reservoirs utilizing non-condensable gases like methane, nitrogen and carbon dioxide and condensable hydrocarbons like propane. A numerical process model was developed, simulated, and calibrated with the help of experimental data.

Lab scale experiments were performed in a cylindrical replica (i.e., physical model) of an oil reservoir with a set of parallel horizontal injection and production wells. The physical model equipped with a grid of thermocouples was packed with synthetic sand saturated with dead oil from Waseca formation. The permeability and porosity of the sand matrix were 10.7 Darcy and $32 \%$, respectively. All the experiments were performed under isothermal conditions at $195^{\circ} \mathrm{C}$ and 1.45 MPa, gauge. Four different gaseous solvents - carbon dioxide, propane, nitrogen and 
methane-were tested at different concentrations of 5.0, 7.5 and $10.0 \mathrm{wt} \%$. A baseline experiment with steam only was also performed.

From this study, it was found that for low pressure reservoirs, hybrid SAGD performed much better than conventional SAGD. The oil recoveries from the addition of solvents were at least 18 percentage points higher than from the steam only experiments. The lower-molecular-weight solvents, i.e., methane and nitrogen, performed better than the higher-molecular-weight solvents, i.e., propane and carbon dioxide.

On an equal weight percentage basis, co-injection of methane and steam resulted in the highest oil recovery of $50.7 \%$ of the original oil in place (OOIP). The solvent retention for propane was the highest, up to $15 \%$, among all the tested solvents. A higher solvent retention can negatively impact the process economics.

For the experiments with co-injection of carbon dioxide and propane with steam, the lowest concentration of $5.0 \mathrm{wt} \%$ of the injected mixture resulted in the highest oil recoveries of 36.5 and $42.6 \%$ OOIP, respectively. For the experiments with co-injection of nitrogen and methane with steam, the highest concentration of $10.0 \mathrm{wt} \%$ of the injected mixture resulted in the highest oil recoveries of 44.2 and $50.7 \%$ OOIP, respectively.

The following are the important conclusions from this study:

1. For low pressure reservoirs, hybrid SAGD performed much better than conventional SAGD. 
2. The oil recoveries using the solvents were at least 18 percentage points higher than that obtained with steam alone.

3. The lower molecular weight solvents, i.e., methane and nitrogen, performed better than the higher molecular weight solvents, i.e., propane and carbon dioxide.

4. On an equal weight percentage basis, addition of methane resulted in the highest oil recovery of $50.7 \%$ of the original oil in place (OOIP).

5. The lowest solvent concentration of $5.0 \mathrm{wt} \%$ resulted in the highest oil recoveries of 36.5 and $42.6 \%$ OOIP, respectively, with carbon dioxide and propane.

6. The highest concentration of $10.0 \mathrm{wt} \%$ resulted in the highest oil recoveries of 44.2 and $50.7 \%$ OOIP, respectively, with nitrogen and methane.

7. Of the four co-injected solvents, propane was found to have highest retention in the physical model, up to $15 \%$. Thus, among the four solvents, the use of propane negatively affects the process economics the most.

8. A numerical model was developed of the hybrid SAGD process, and its recovery predictions were history-matched to experimental recoveries obtained with carbon dioxide, nitrogen and methane.

9. Numerical simulations were performed for the solvents that were found to be promising. The predictions of the calibrated model had relative errors less than $5.0 \%$.

The findings of this study suggest that hybrid SAGD with methane or nitrogen seems to be a viable oil process for low-pressure reservoirs. The calibrated model predicts oil recovery with a reasonably high accuracy, and is a promising tool to help with process optimization and field scale predictions. 


\subsection{Recommendations}

The experimental work can be extended to assess the hybrid SAGD performance as follows:

1. Many combinations of solvent mixtures (of methane, propane, butane, pentane, carbon dioxide, etc.) can be tested close to the dew point at operating conditions.

2. Experiments can be designed to inject a mixture of hot flue gases and steam generated from the direct contact steam generators.

The numerical model calibrated in this work may be used to:

1. Investigate applicability of the low-pressure SAGD field performance.

2. Conduct a sensitivity and optimization study to improve the performance of hybrid SAGD processes. 


\section{Appendix A: Model Temperature Plots}

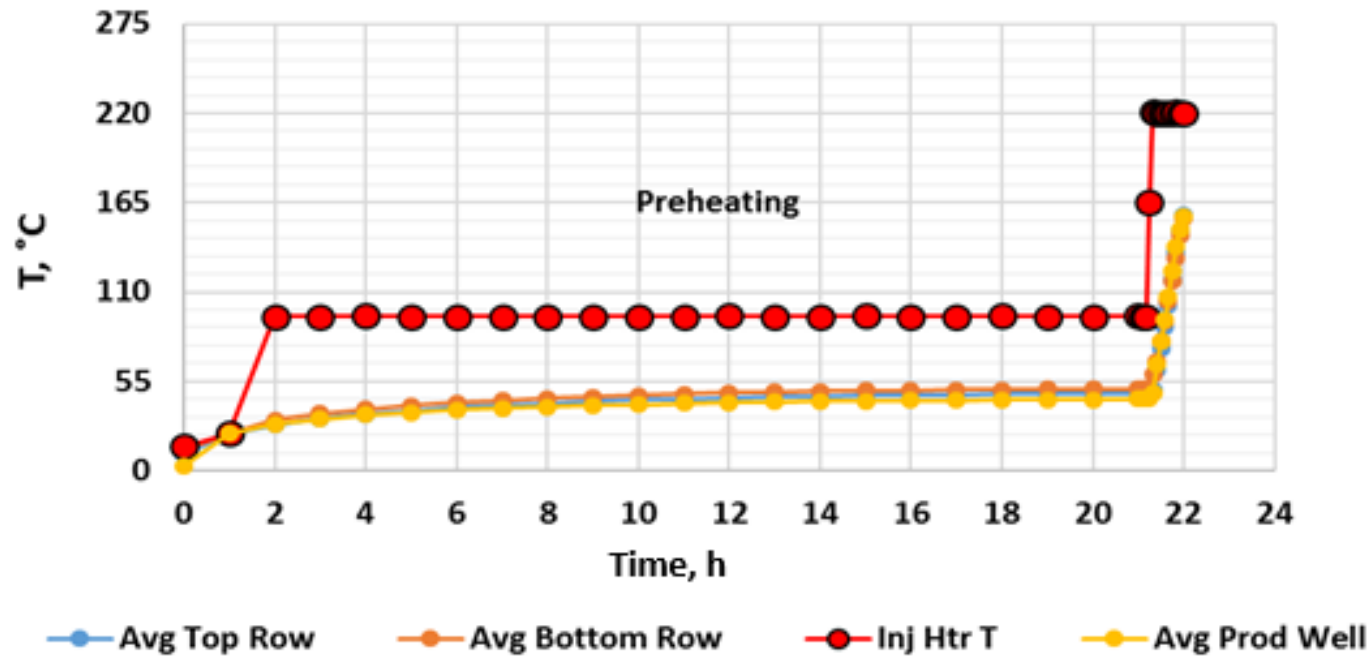

Figure A.1 Baseline experiment preheating model temperatures

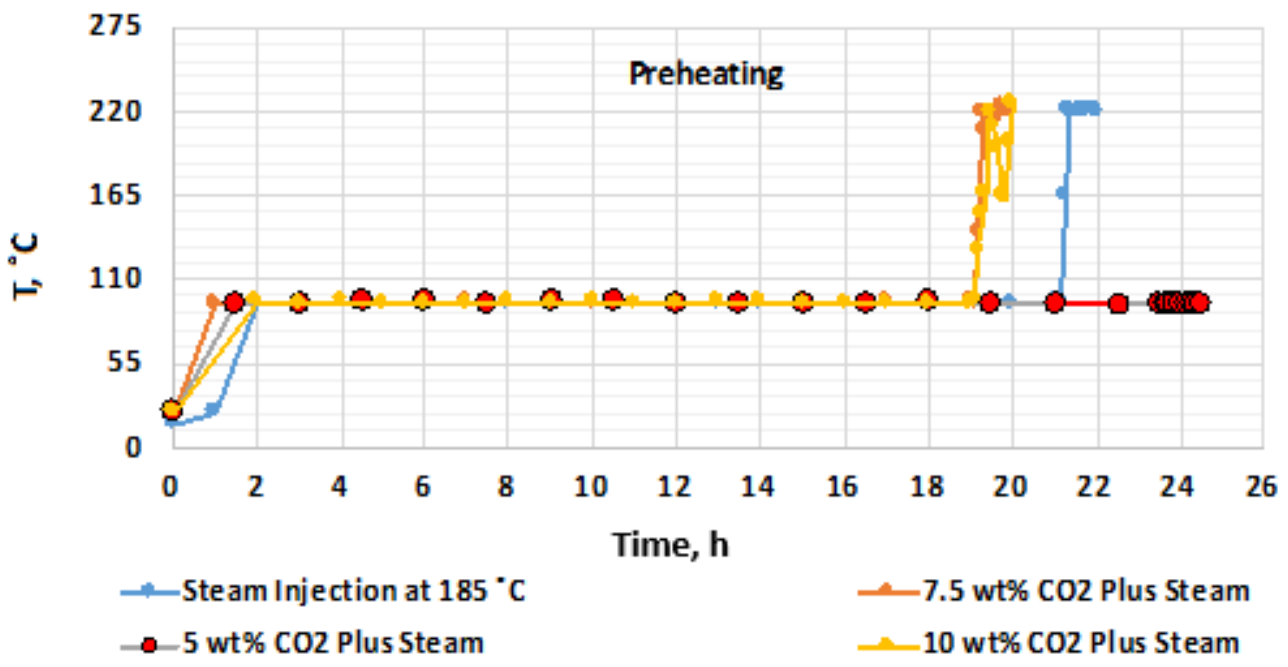

Figure A.2 Steam plus $5.0 \mathrm{wt} \% \mathrm{CO}_{2}$ Preheating model temperatures 


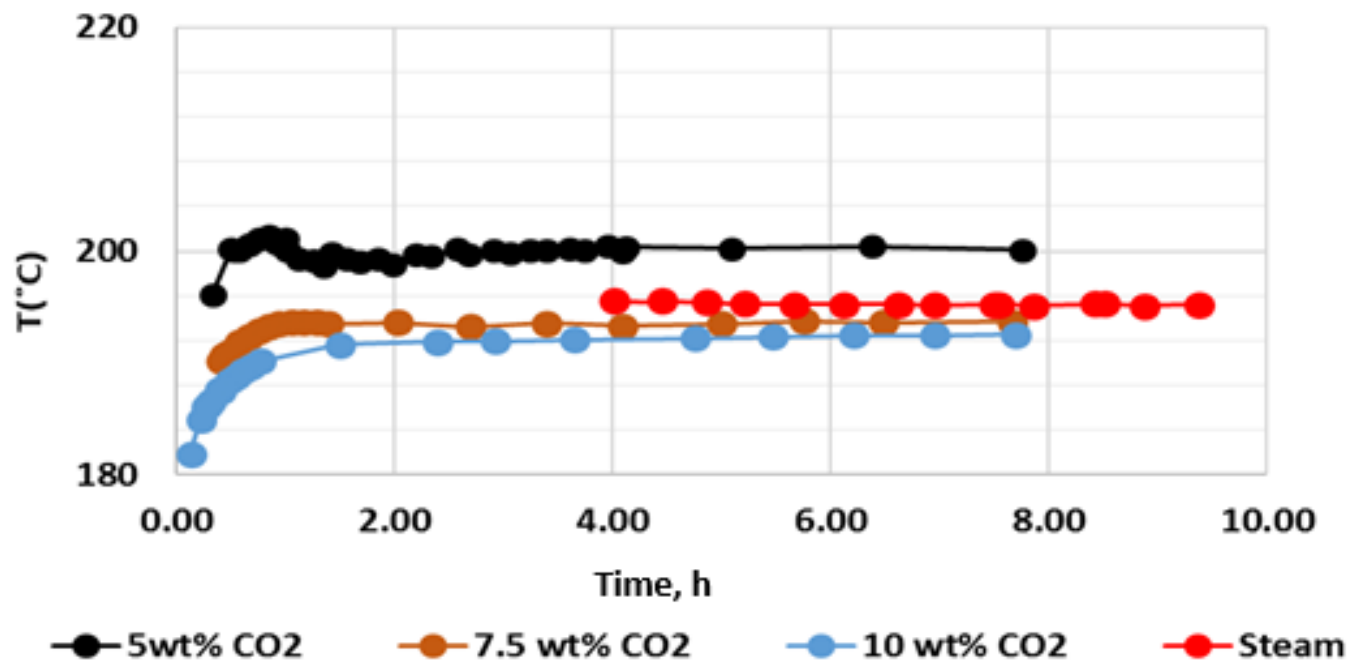

Figure A.3 Steam plus $\mathrm{CO}_{2}$ top row temperatures

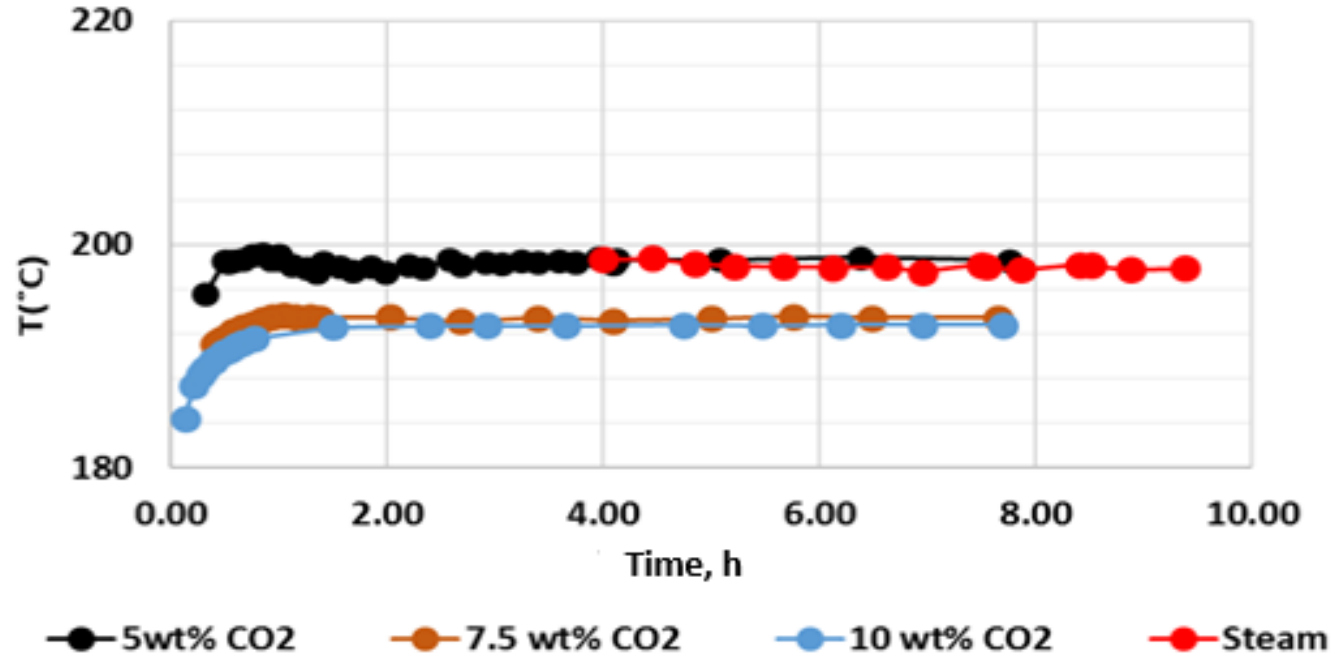

Figure A.4 Steam plus $\mathrm{CO}_{2}$ bottom row temperatures 


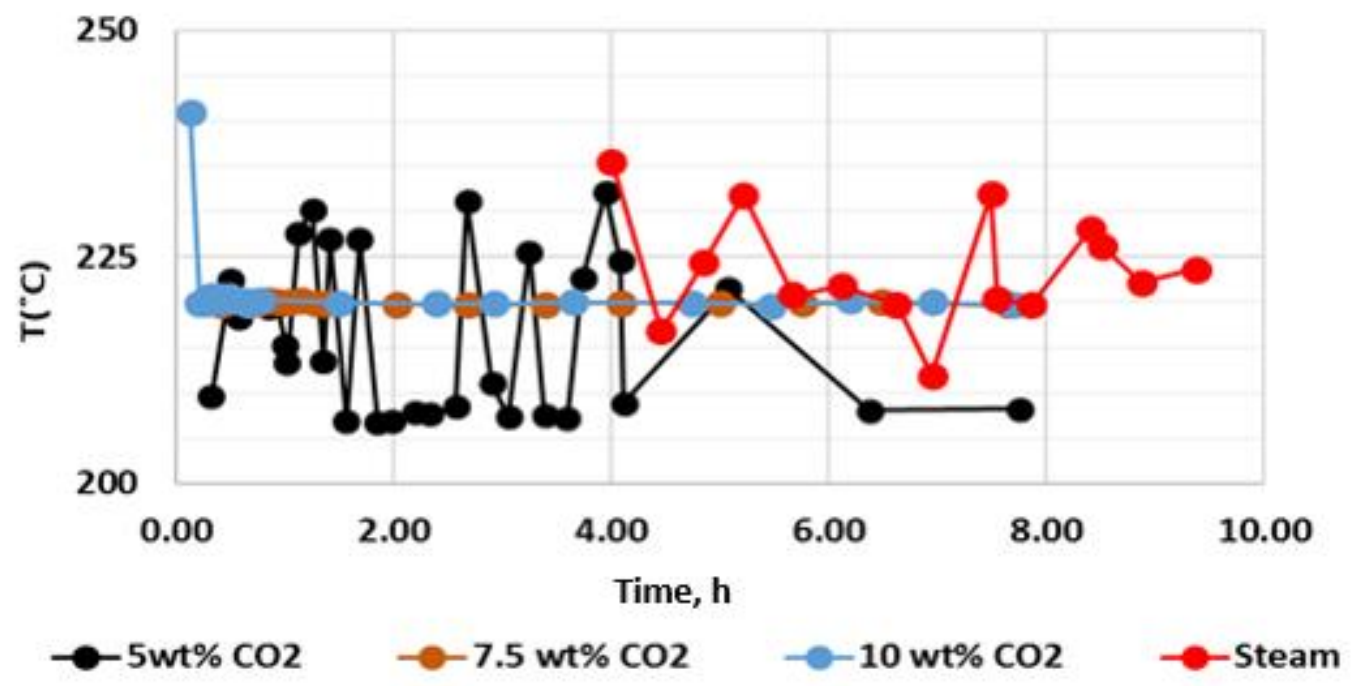

Figure A.5 Steam plus $\mathrm{CO}_{2}$ injection well temperatures

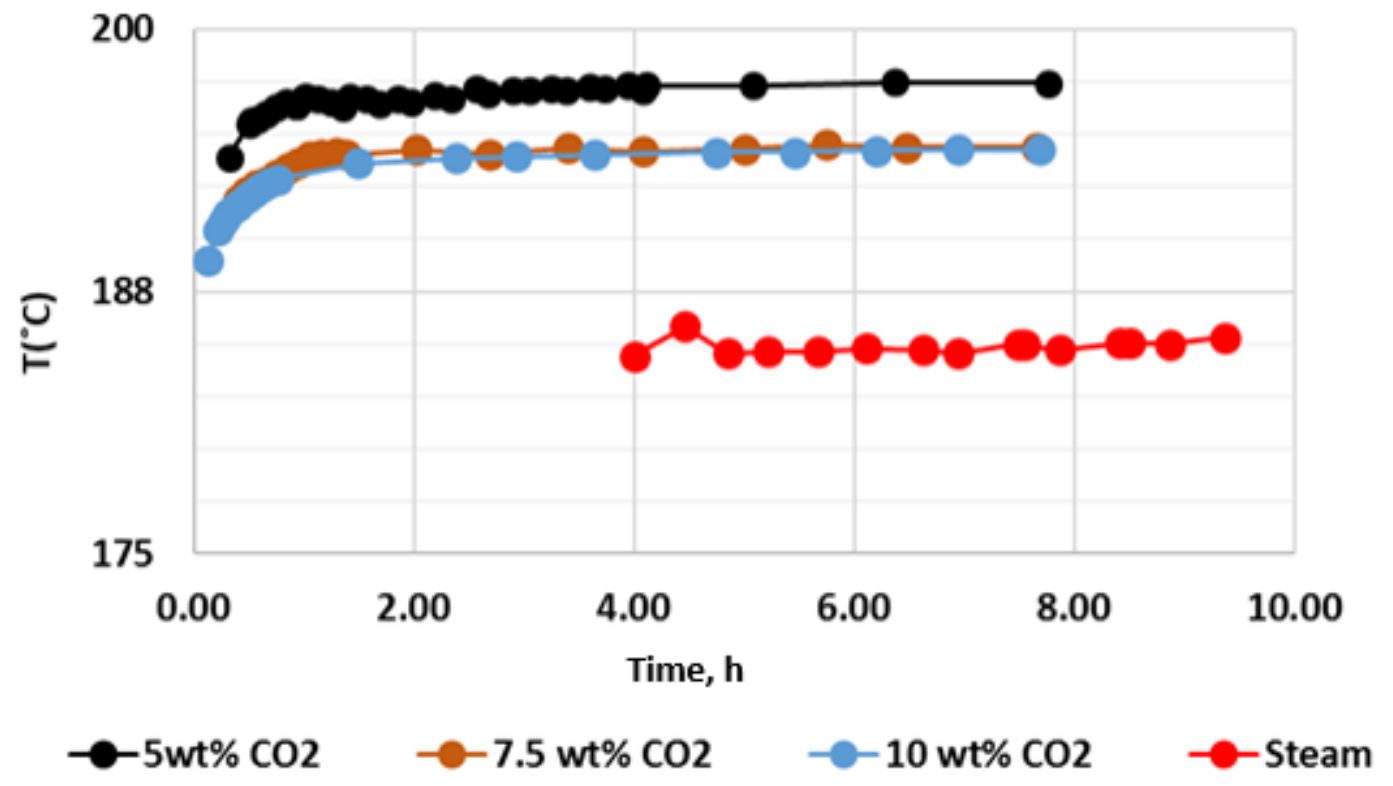

Figure A.6 Steam plus $\mathrm{CO}_{2}$ production well temperatures 


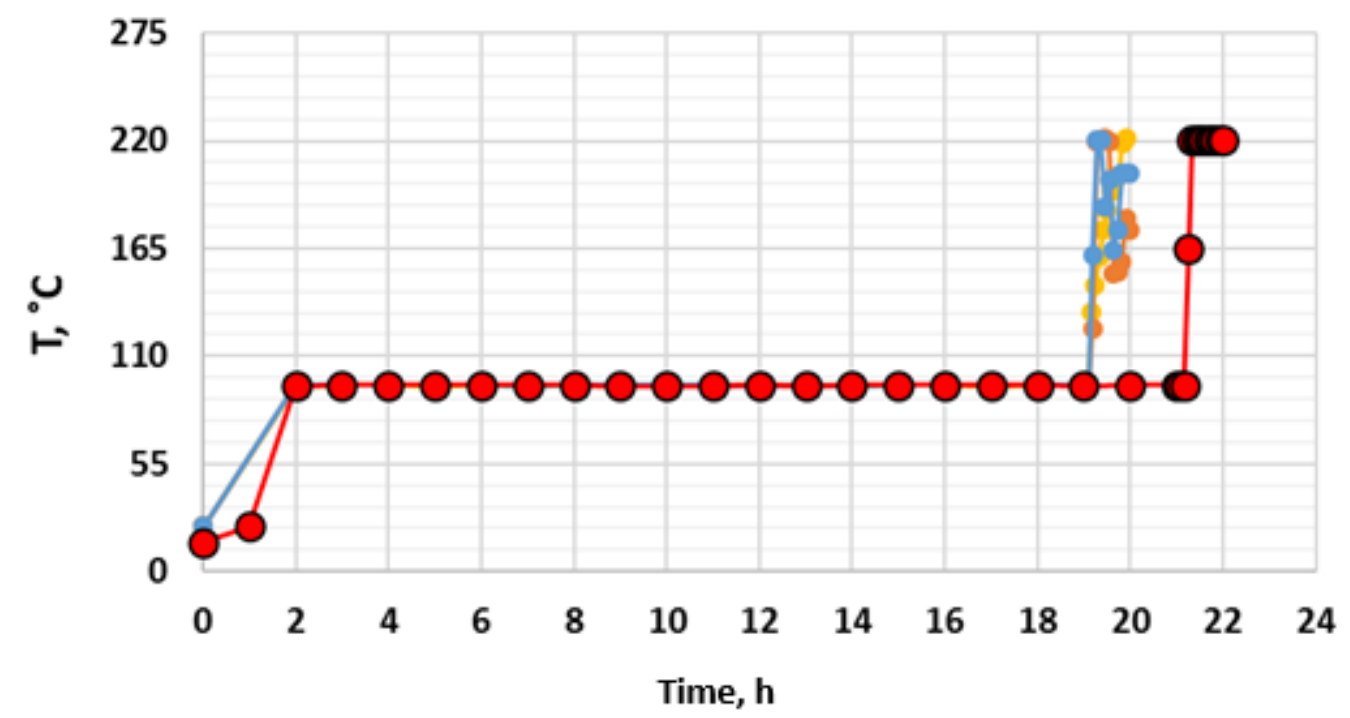

-5 wt\% N2 Plus Steam --7.5 wt\% N2 Plus Steam $-\bullet 10$ wt\% N2 Plus Steam $-0-$ Steam

Figure A.7 Steam plus $\mathrm{N}_{2}$ preheating model temperatures

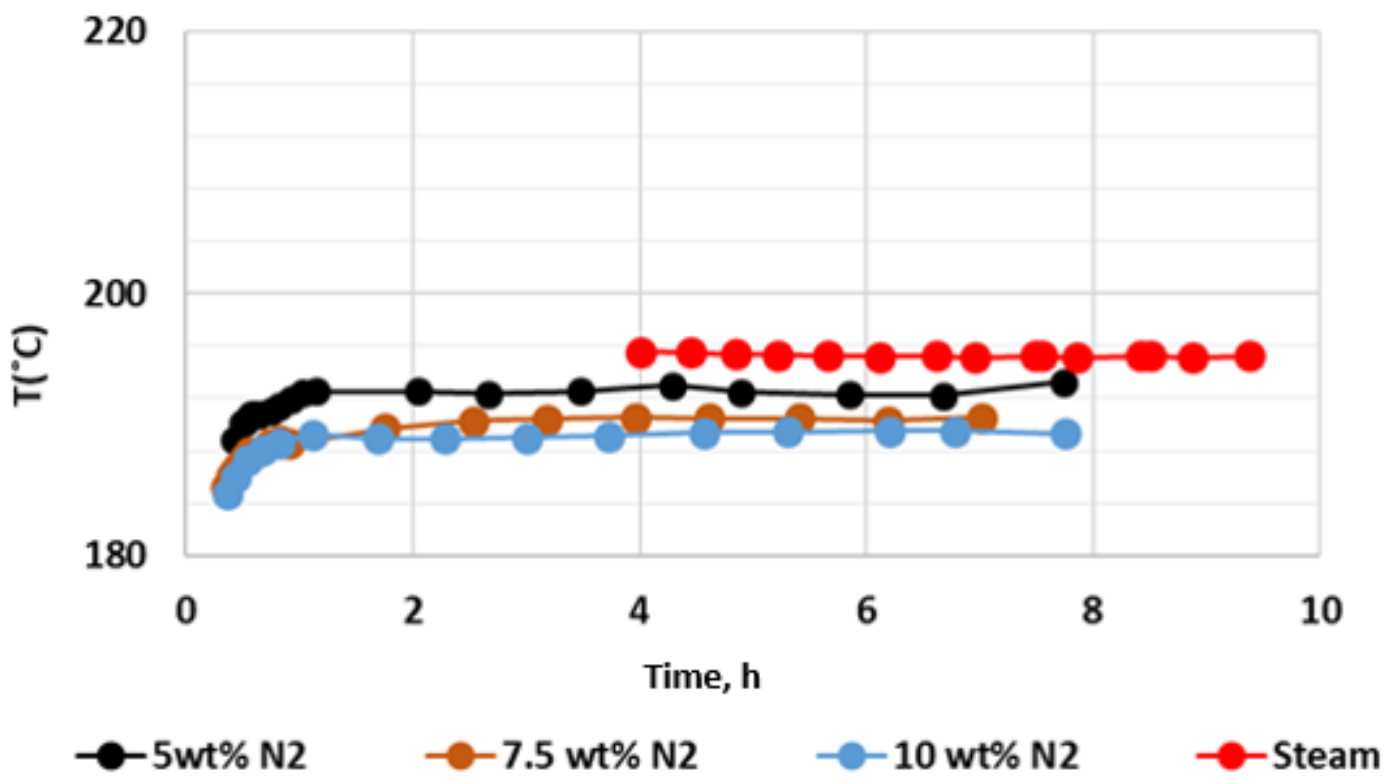

Figure A.8 Steam plus $\mathrm{N}_{2}$ top row temperatures 


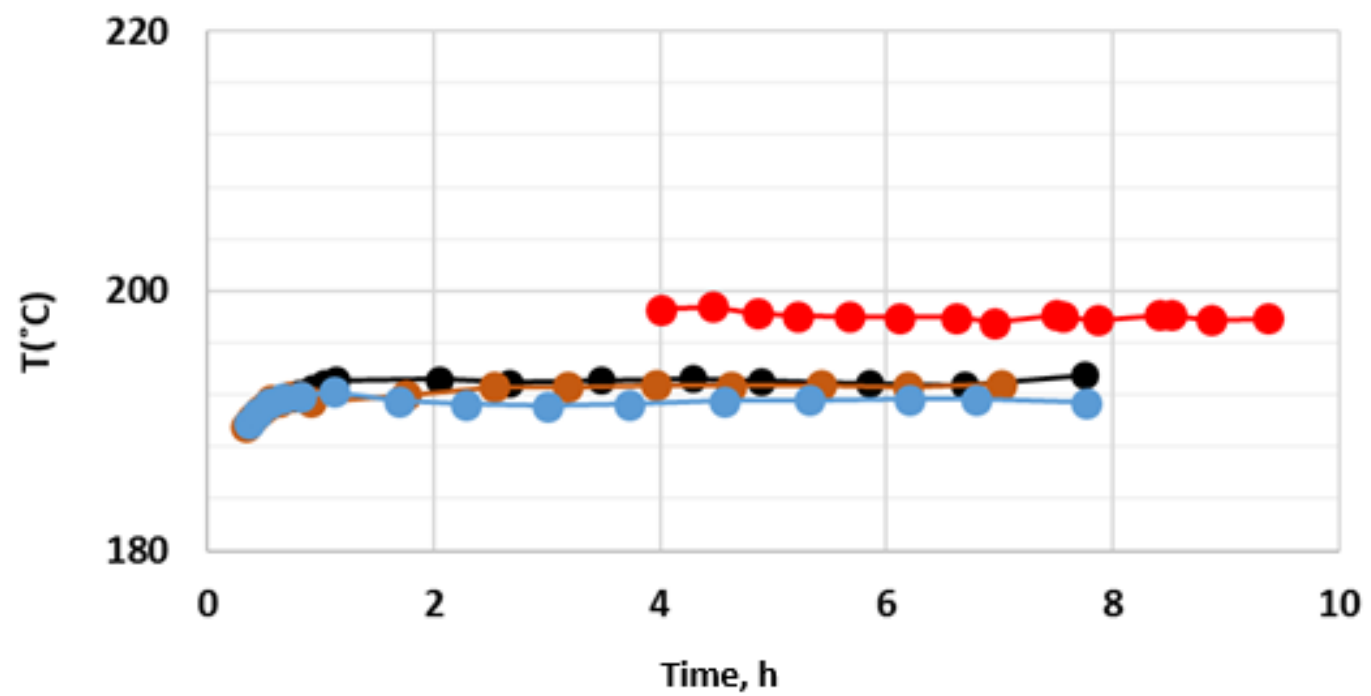

$\multimap-5 w t \%$ N2 -7.5 wt\% N2 $-0-10$ wt\% N2 - -Steam

Figure A.9 Steam plus $\mathrm{N}_{2}$ bottom row temperatures

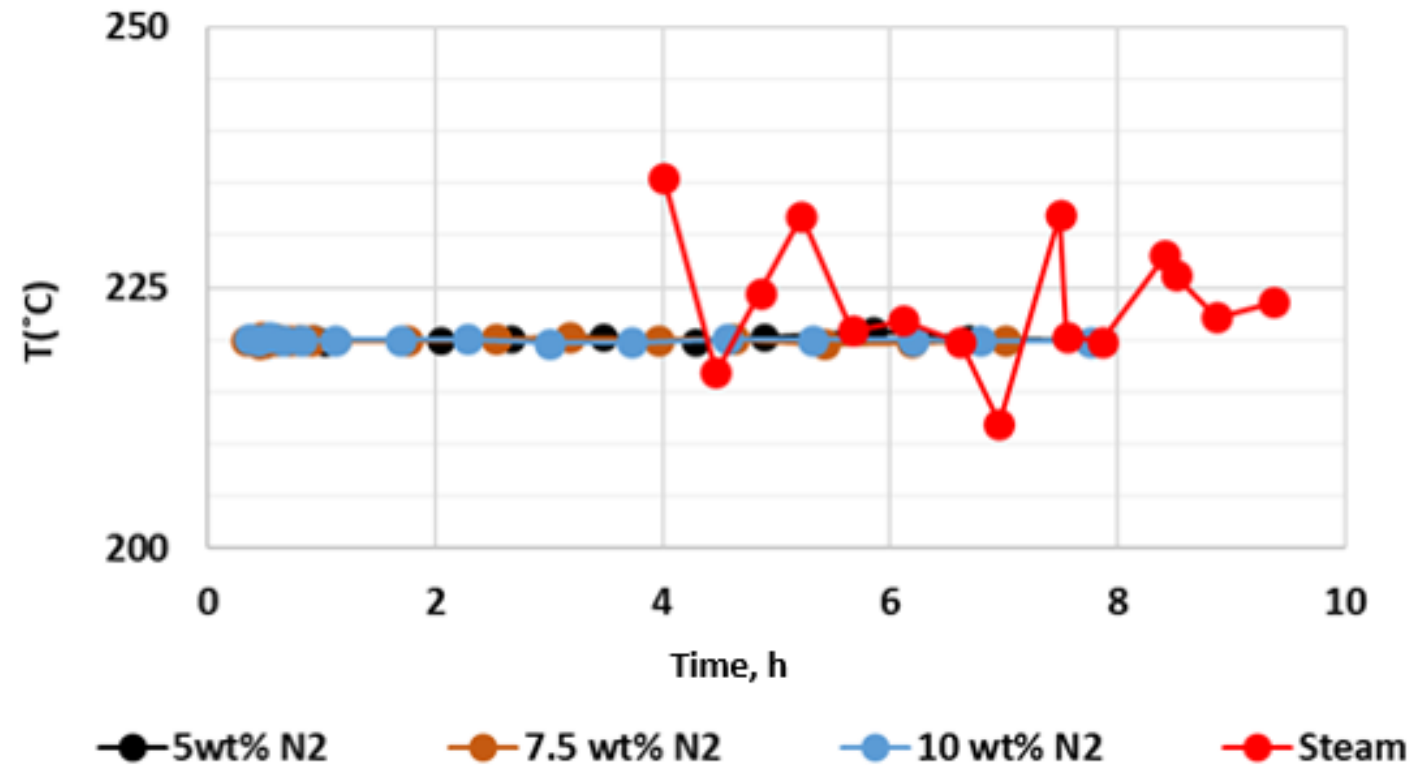

Figure A.10 Steam plus $\mathrm{N}_{2}$ injection well temperatures 


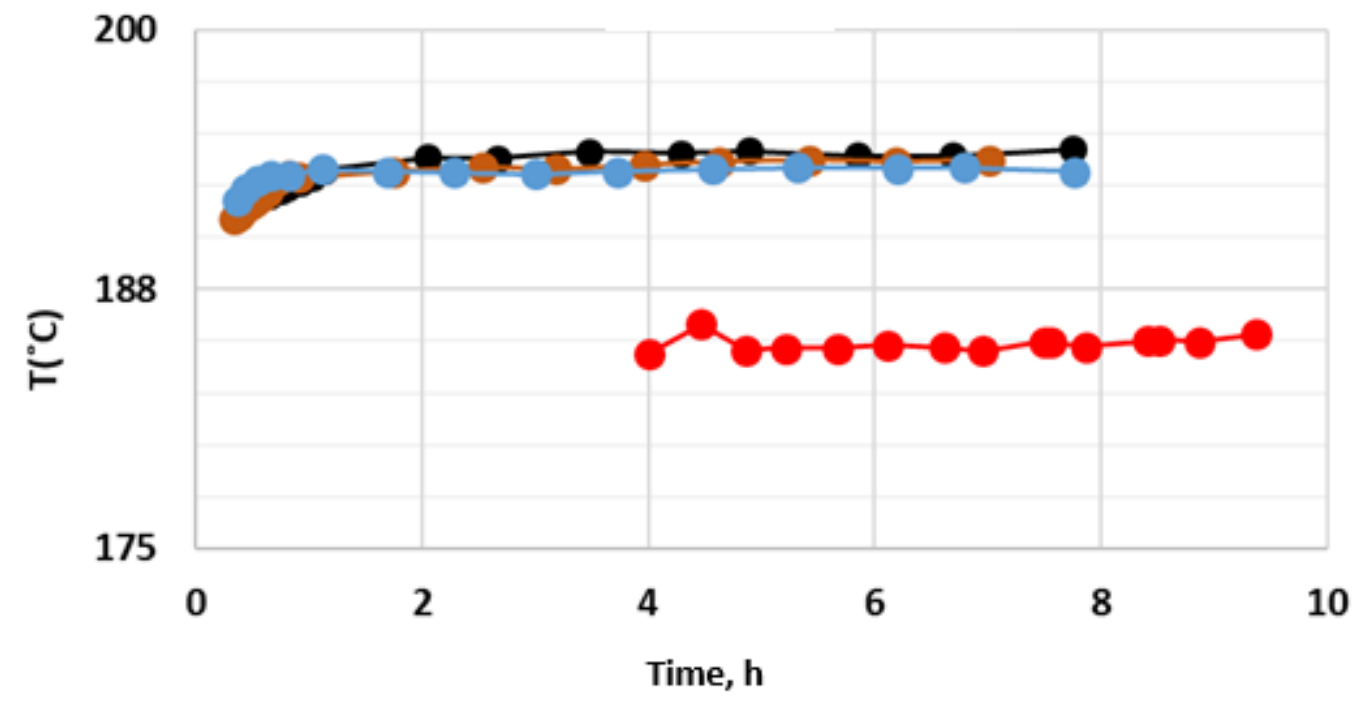

$\multimap-5 w t \%$ N2 -7.5 wt\% N2 -10 wt\% N2 - -Steam

Figure A.11 Steam plus $\mathrm{N}_{2}$ production well temperatures

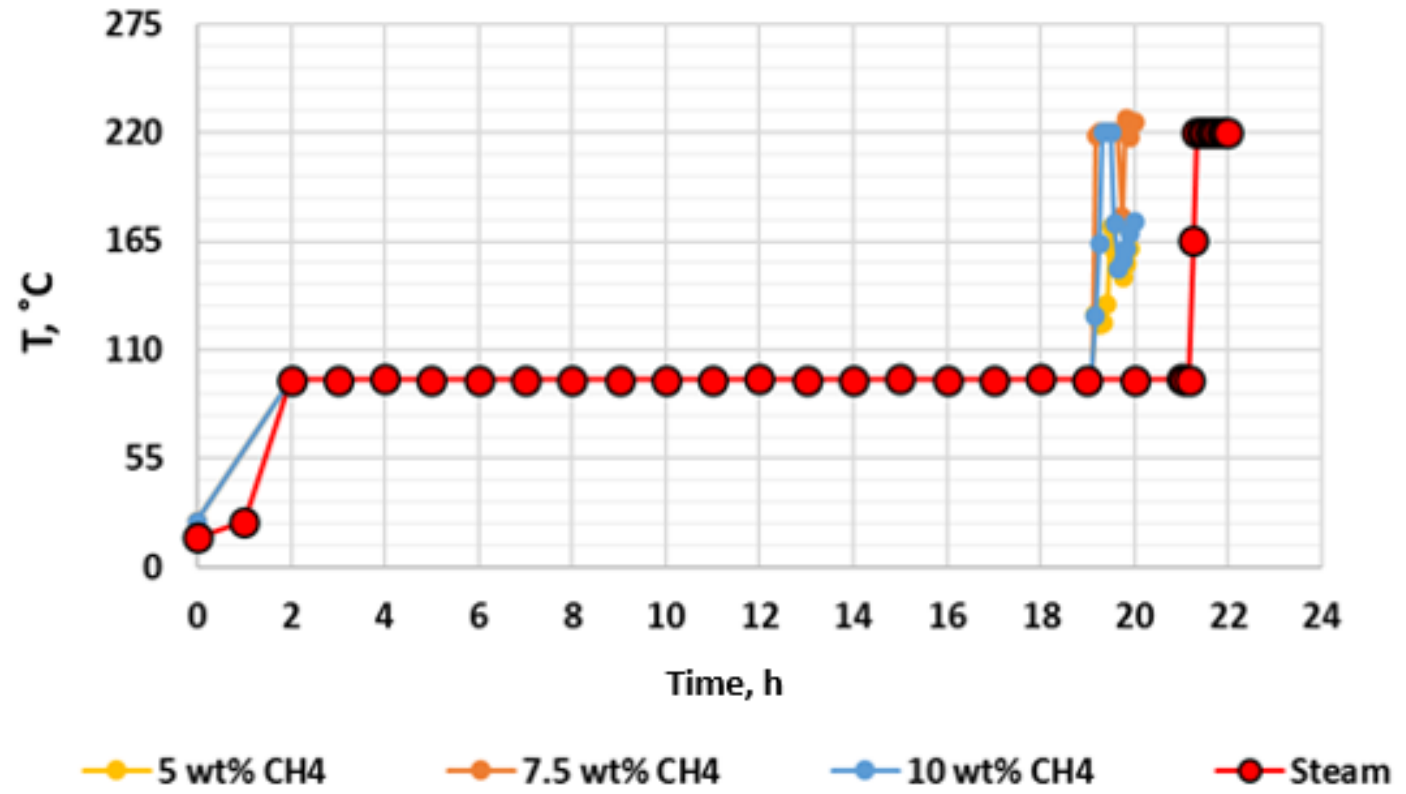

Figure A.12 Steam plus $\mathrm{CH}_{4}$ model preheating temperatures 


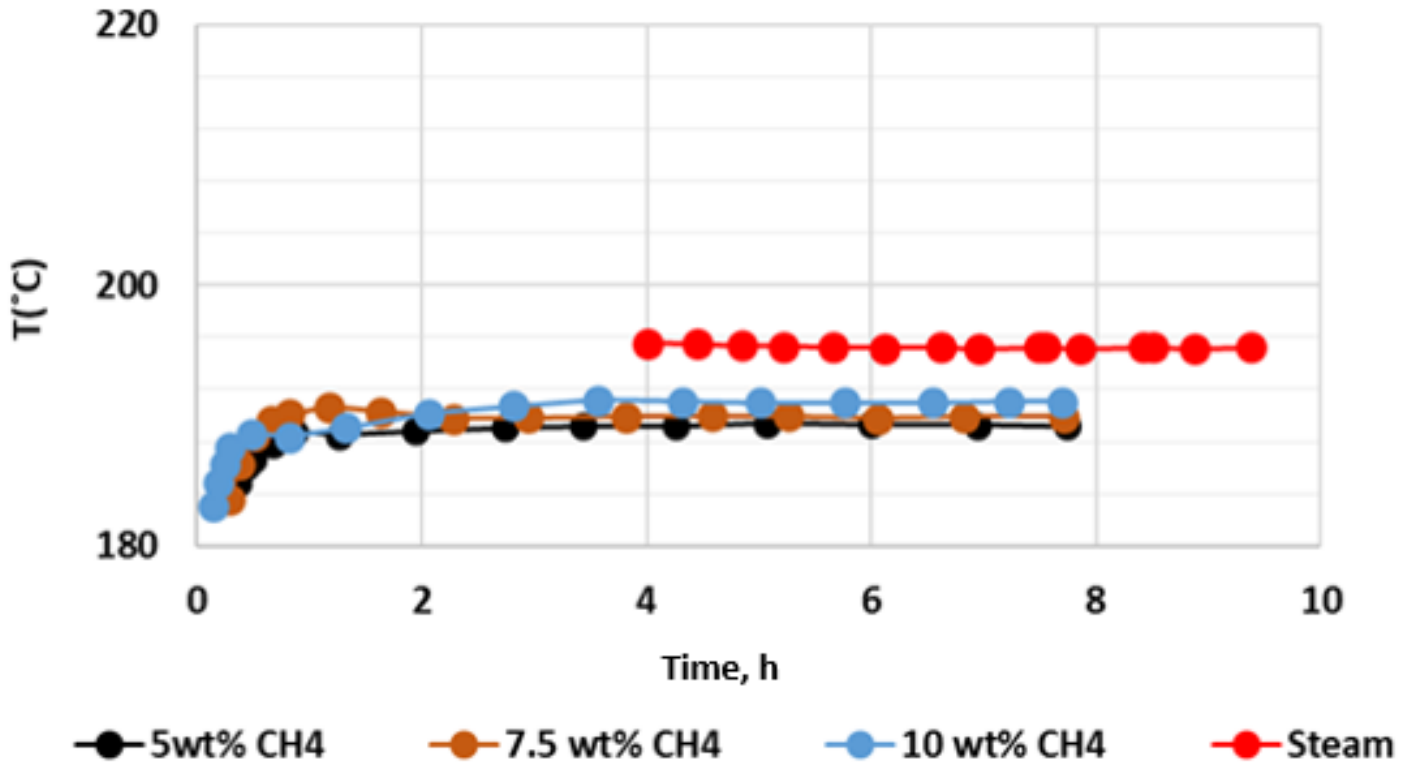

Figure A.13 Steam plus $\mathrm{CH}_{4}$ top row temperatures

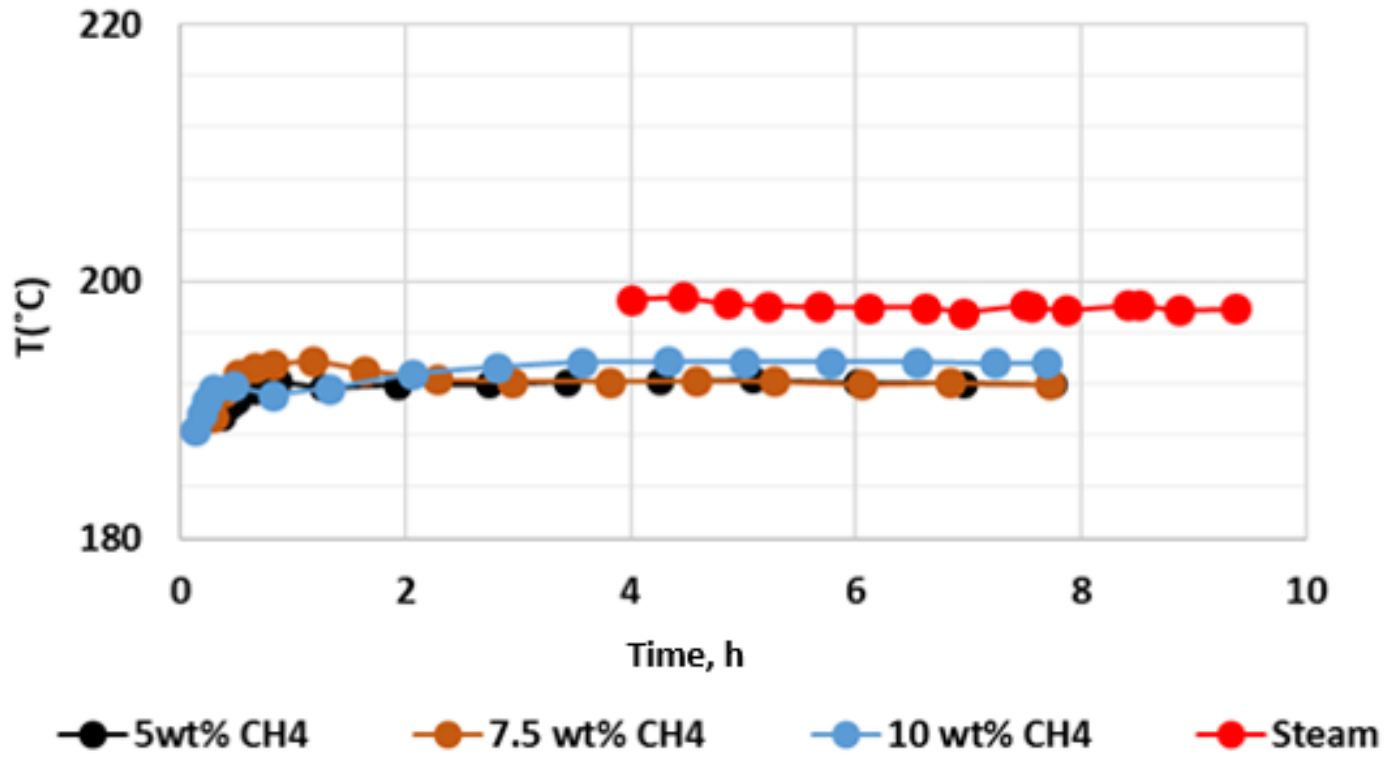

Figure A.13 Steam plus $\mathrm{CH}_{4}$ bottom row temperatures 


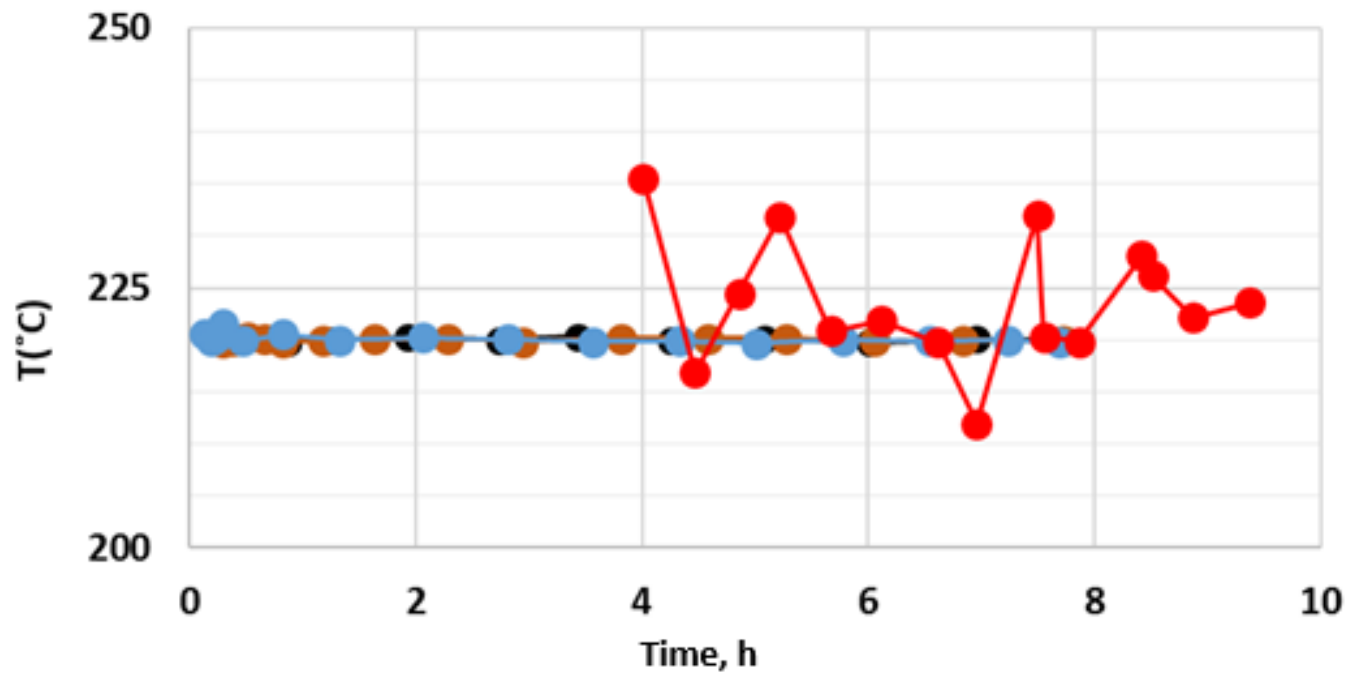

$5 w t \%$ CH4

-7.5 wt\% $\mathrm{CH} 4$

10 wt $\%$ CH4

Steam

Figure A.14 Steam plus $\mathrm{CH}_{4}$ injection well temperatures

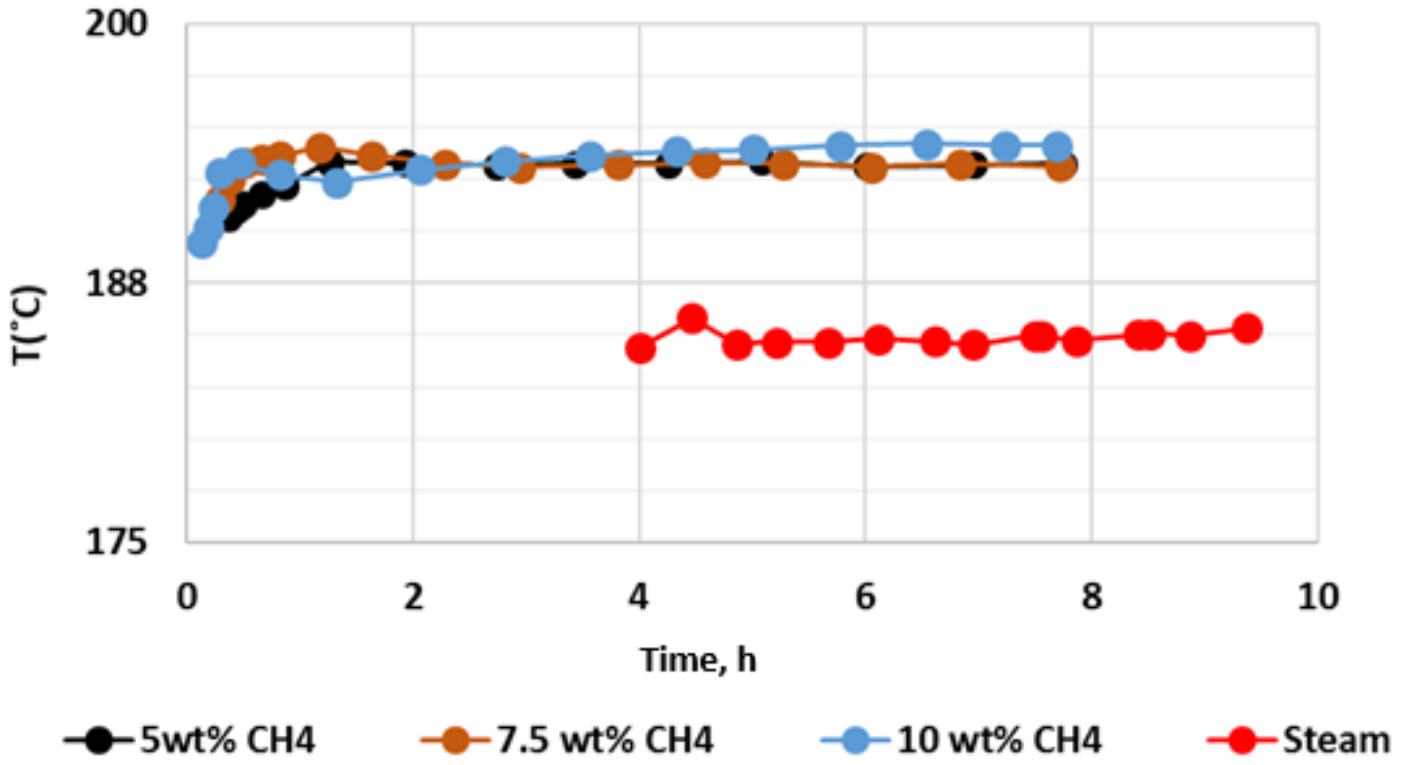

Figure A.15 Steam plus $\mathrm{CH}_{4}$ production well temperatures 


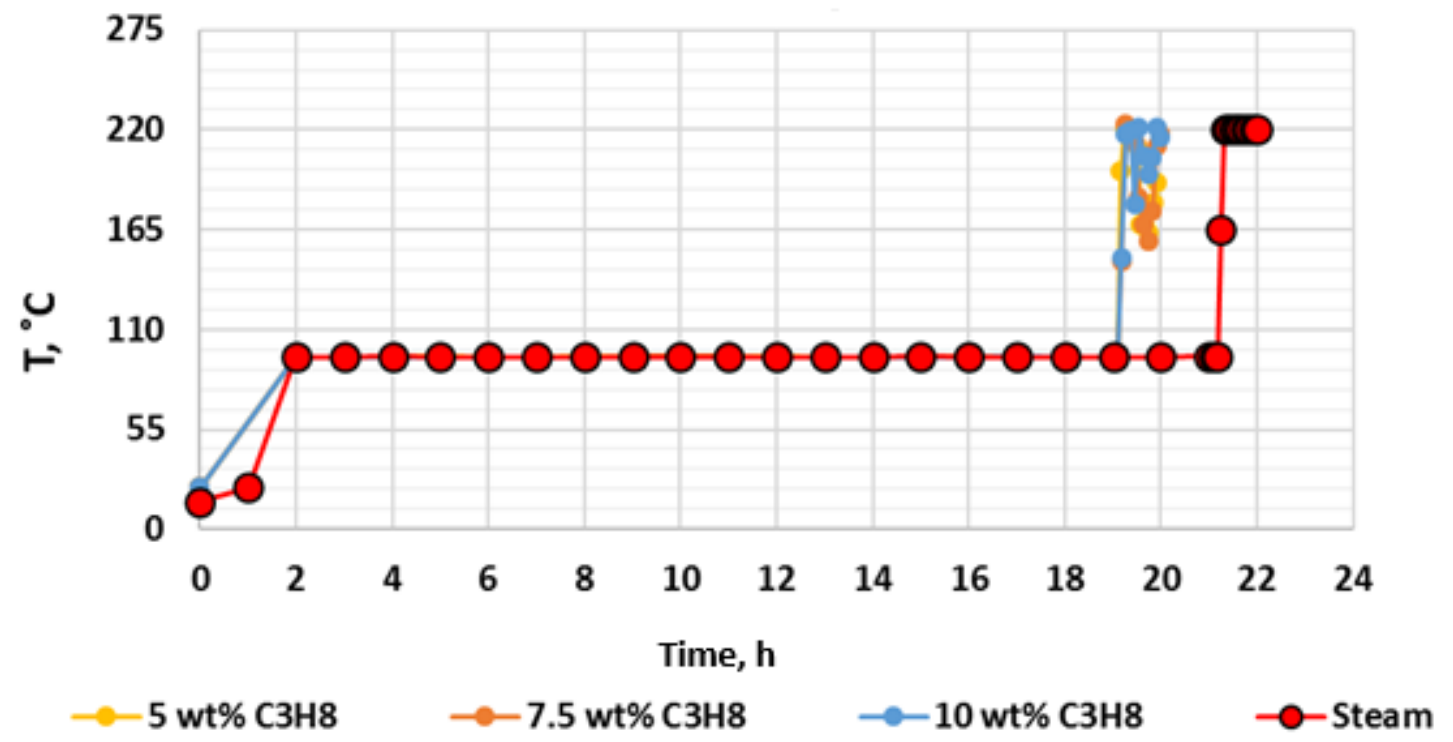

Figure A.16 Steam plus $\mathrm{C}_{3} \mathrm{H}_{8}$ preheating model temperatures

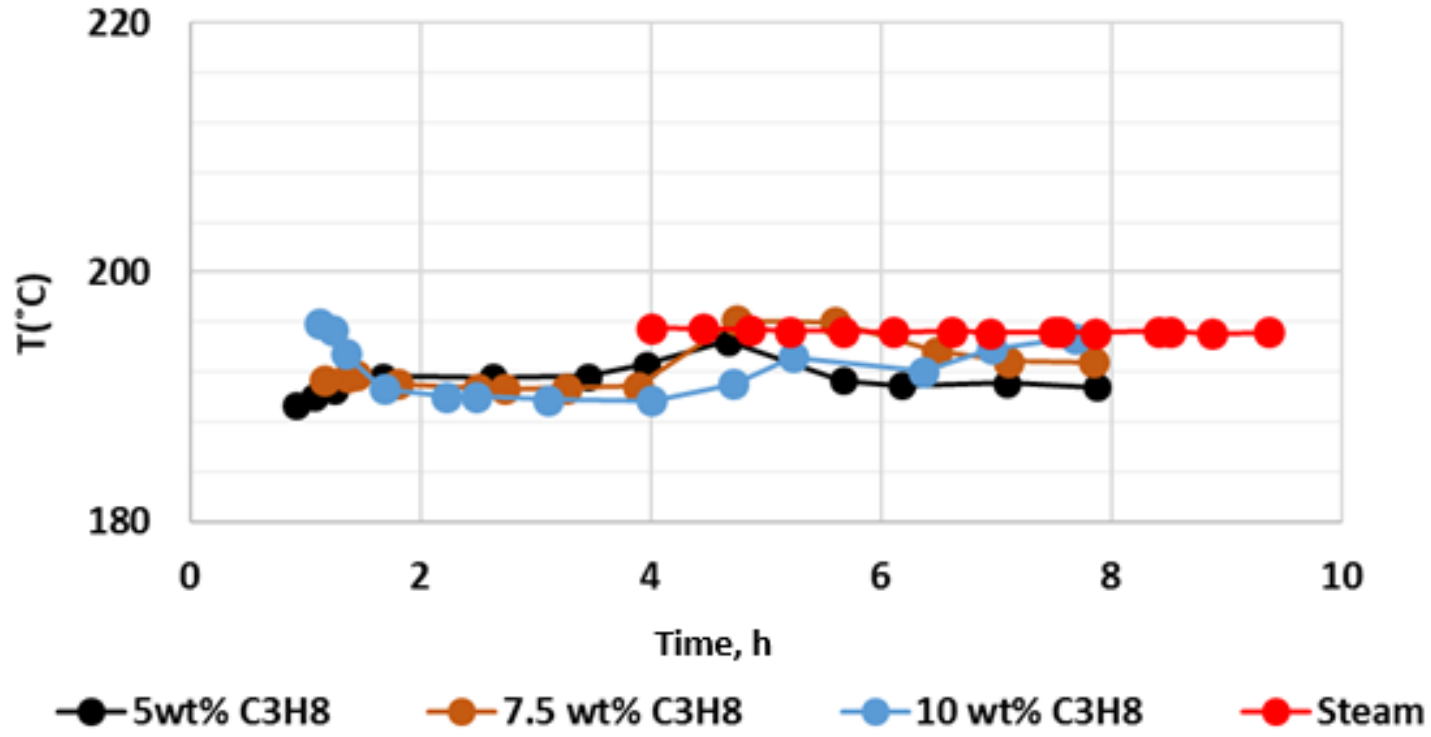

Figure A.17 Steam plus $\mathrm{C}_{3} \mathrm{H}_{8}$ top row temperatures 


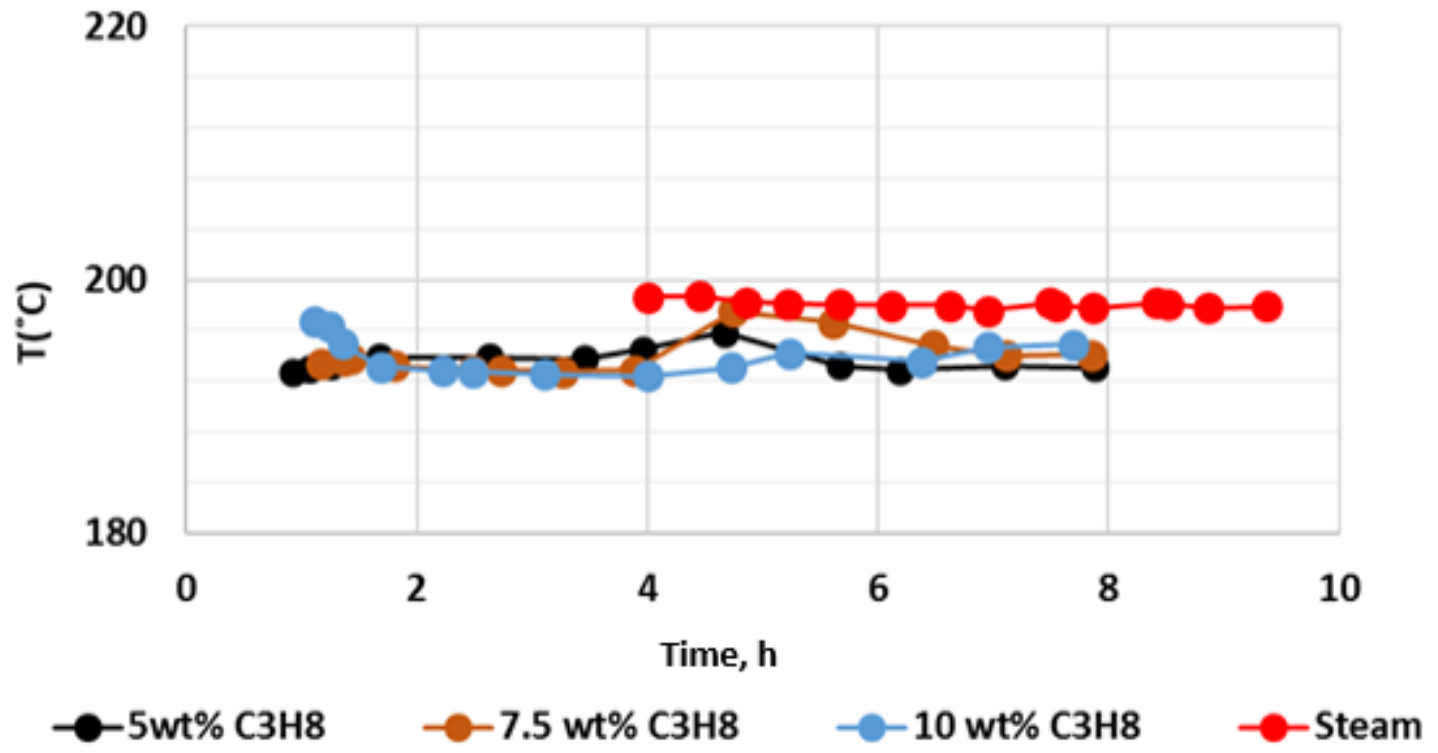

Figure A.18 Steam plus $\mathrm{C}_{3} \mathrm{H}_{8}$ bottom row temperatures

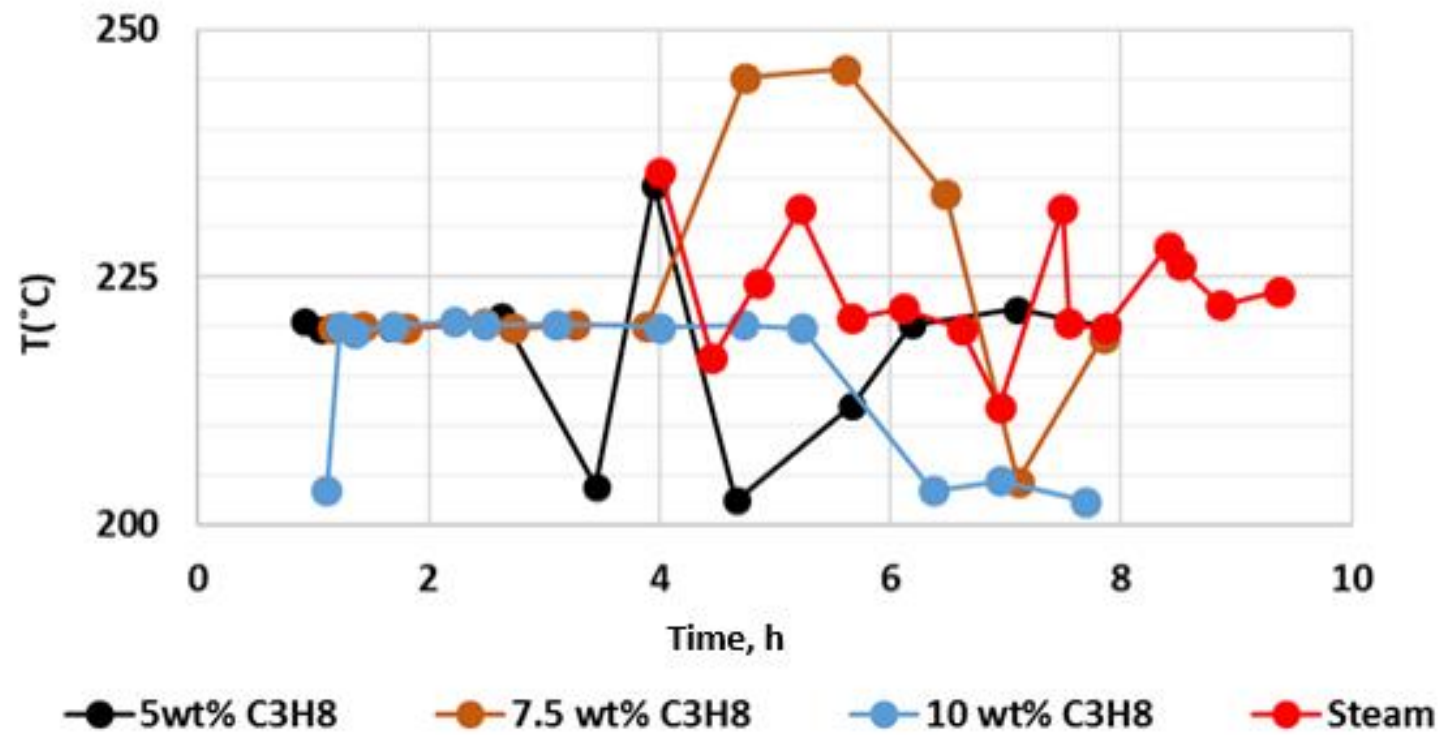

Figure A.19 Steam plus $\mathrm{C}_{3} \mathrm{H}_{8}$ injection well temperatures 


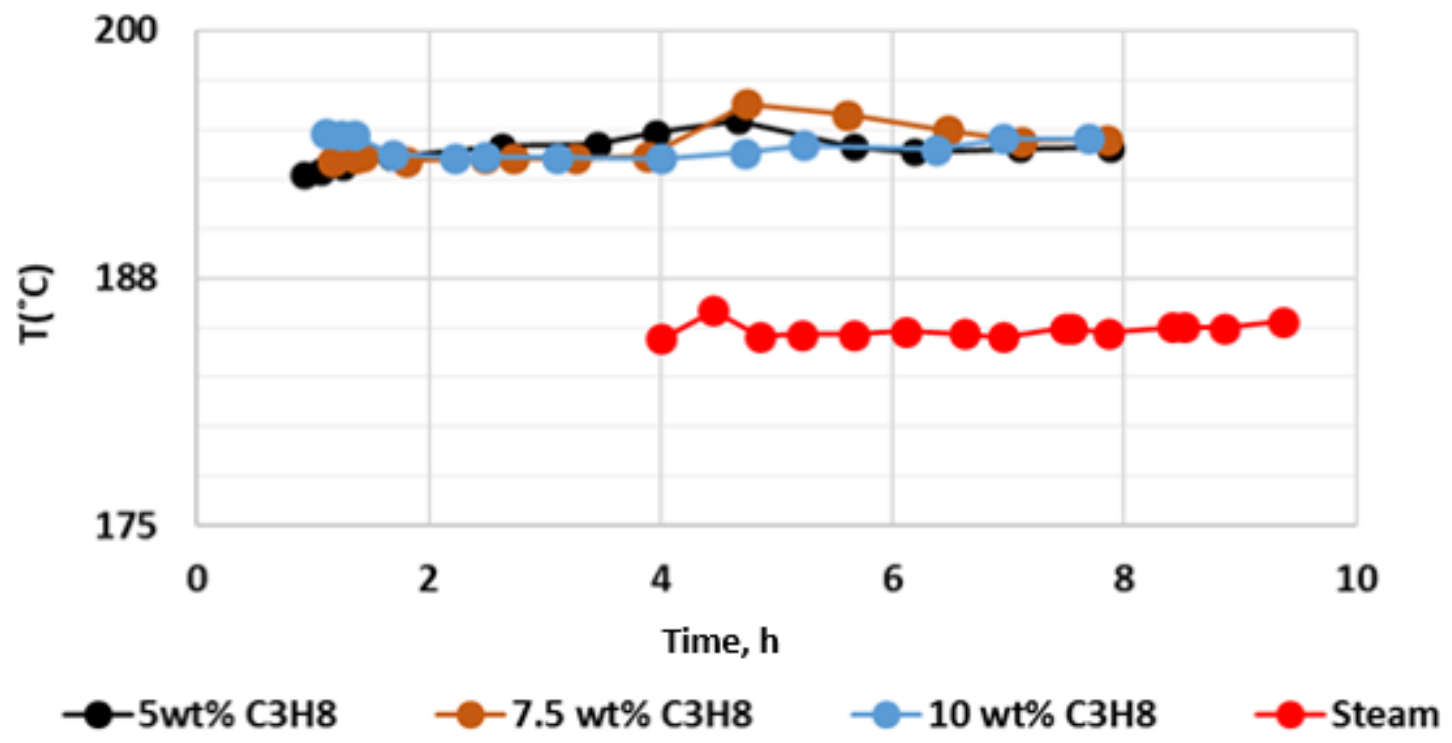

Figure A.20 Steam plus $\mathrm{C}_{3} \mathrm{H}_{8}$ production well temperatures 


\section{Appendix B: WinProp Fundamental \\ Equations}

The following are the fundamental equations used in CMG's WinProp software:

$P=\frac{\mathrm{R} T}{v-b}-\frac{a}{v^{2}+v b(1+c)-c b^{2}}$

Or

$P=\frac{\mathrm{R} T}{v-b}-\frac{a}{\left(v+\delta_{1} b\right)\left(v+\delta_{2} b\right)}$

where

$P=$ pressure, $\mathrm{kPa}$

$\mathrm{R}=$ gas constant, $\mathrm{j} /($ mol Kelvin $)$

$T=$ temperature, ${ }^{\circ} \mathrm{C}$

$2 \delta_{1}=(1+c)-\sqrt{(1+c)^{2}+4 c}$

$\delta_{1} \delta_{2}=-c$

When $c=1$, the equation is the Peng-Robisnon EOS, and when $c=0$, it becomes the Soave-RedlickKwong EOS.

For the pure components the factors $\mathrm{a}$ and $\mathrm{b}$ are expressed in terms of the critical properties and the acentric factors.

$\sqrt{a}=\sqrt{a_{c} \alpha}$ 
$\sqrt{a_{c}}=\sqrt{\Omega_{a}}\left[\frac{R \mathrm{~T}_{c}}{\mathrm{P}_{c}}\right]$

$\sqrt{\alpha}=1+k\left[1-\sqrt{\frac{T}{\mathrm{~T}_{c}}}\right]$

$b=\Omega_{b} R\left[\frac{R \mathrm{~T}_{c}}{\mathrm{P}_{c}}\right]$

The EOS is defined as:

$z^{3}-z^{2}(1-c B)+z\left[A-B(1+c)-B^{2}(1+2 c)\right]-\left[A B-c\left(B^{3}+B^{2}\right)\right]=0$

where

$\mathrm{Z}=$ the compressibility factor

$\mathrm{P}_{\mathrm{c}}=$ critical pressure, $\mathrm{KPa}$

$\mathrm{T}_{\mathrm{c}}=$ critical temperature, ${ }^{\circ} \mathrm{C}$

$\Omega_{a}=$ interaction coefficient

$\Omega_{b}=$ interaction coefficient

$A=\frac{a p}{R T^{2}}$

$B=\frac{b p}{R T}$

and for the Peng-Robinson EOS

$k=0.37464+1.54226 \omega-0.26992 \omega^{2}$

For heavier than n-decane

$k=0.379642+1.48503 \omega-0.164423 \omega^{2}+0.016666 \omega^{3}$ 


\section{Appendix C: K-Value, Density and Viscosity Data for Fluid Model Tuning}

Table C.1 Carbon dioxide-oil experimental data

\begin{tabular}{ccccc}
$\boldsymbol{T}\left({ }^{\circ} \boldsymbol{C}\right)$ & $\mathbf{P}$ & K-Value & Density & Viscosity \\
\cline { 2 - 5 } & $\mathbf{k P a}$ & Ratio & $\mathbf{K g} / \mathbf{m}^{\mathbf{3}}$ & $\mathbf{~} \mathbf{m a} \cdot \mathbf{s}$ \\
\hline $\mathbf{2 1}$ & 500 & 12.41 & 983.3 & 9591 \\
\hline $\mathbf{2 1}$ & 1500 & 5.711 & 983.8 & 4564 \\
\hline $\mathbf{2 1}$ & 3500 & 2.635 & 985.2 & 1023 \\
\hline $\mathbf{3 5}$ & 500 & 5.420 & 967.8 & 2517 \\
\hline $\mathbf{3 5}$ & 1550 & 1.315 & 967.4 & 1291 \\
\hline
\end{tabular}

Table C.2 Propane-oil experimental data

\begin{tabular}{ccccc}
$\boldsymbol{T}\left({ }^{\circ} \boldsymbol{C}\right)$ & $\mathbf{P}$ & K-Value & Density & Viscosity \\
\cline { 2 - 5 } & $\mathbf{k P a}$ & Ratio & $\mathbf{K g} / \mathbf{m}^{\mathbf{3}}$ & $\mathbf{m P a} \cdot \mathbf{s}$ \\
\hline $\mathbf{2 1}$ & 500 & 1.825 & 915.4 & 204.3 \\
\hline $\mathbf{2 1}$ & 700 & 1.408 & 851.5 & 22.60 \\
\hline $\mathbf{3 5}$ & 350 & 1.403 & 833.9 & 12.80 \\
\hline $\mathbf{3 5}$ & 600 & 2.127 & 912.1 & 116.0 \\
\hline $\mathbf{3 5}$ & 1000 & 6.613 & 951.8 & 1168
\end{tabular}

Table C.3 Methane-oil experimental data

\begin{tabular}{ccccc}
$\boldsymbol{T}\left({ }^{\circ} \boldsymbol{C}\right)$ & $\mathbf{P}$ & K-Value & Density & Viscosity \\
\cline { 2 - 5 } & $\mathbf{k P a}$ & Ratio & $\mathbf{K g} / \mathbf{m}^{\mathbf{3}}$ & $\mathbf{m P a} \cdot \mathbf{s}$ \\
\hline $\mathbf{2 1}$ & 650 & 27.45 & 983.1 & 14685 \\
\hline $\mathbf{2 1}$ & 2500 & 8.014 & 978.1 & 8971 \\
\hline $\mathbf{2 1}$ & 10000 & 2.985 & 960.9 & 2473 \\
\hline $\mathbf{3 5}$ & 650 & 30.39 & 970.7 & 3317 \\
\hline $\mathbf{3 5}$ & 2500 & 8.762 & 966.4 & 2313 \\
\hline $\mathbf{3 5}$ & 10000 & 3.051 & 949.8 & 853.4
\end{tabular}




\section{References}

Abukhalifeh, H., Lohi, A., and Upreti, S.R. 2009. A Novel Technique to Determine concentration Dependent Solvent Dispersion in Vapex. Energies 2 (4): 851-872.

AER presentation, Scheme 10587, 2015.

AER presentation, Scheme 11596, 2015.

Agrawal, P., Schoeggl, F.F., Satyro, M.A., Taylor, S.D. and Yarranton, H.W. 2012. Measurement and Modeling of the Phase Behavior of Solvent Diluted Bitumens. Fluid Phase Equilibria 334: $51-64$.

Aherne, A.L. and Birrell, G.E. 2002. Observations Relating to Non-Condensable Gases in a Vapour Chamber: Phase B of the Dover Project. Paper presented at the SPE International Thermal Operations and Heavy Oil Symposium and International Horizontal Well Technology Conference. Calgary, Alberta, Canada. SPE-79023-MS.

Akinboyewa, J., Das, S.K., Wu, Y.S., and Kazemi, H. 2010. Simulation of Expanding Solvent Steam Assisted Gravity Drainage in a Field Case Study of a Bitumen Oil Reservoir. Paper presented at the 2010 SPE Improved Oil Recovery Symposium, Tulsa, Oklahoma, 24-28 April. SPE-129963-MS.

Allen, J.C. 1974. Gaseous Solvent Heavy Oil Recovery. US Patent 1027851.

Al-Murayri, M.T., Harding, T.G., and Maini, B.B. 2011. Impact of Non-Condensable Gas on Performance of Steam Assisted Gravity Drainage. Journal of Canadian Petroleum Technology 50 (7-8): 46-54. 
Ardali, M., Barrufet, M., and Mamora D.D. 2012. Effect of Non-Condensable Gas on Solvent Aided SAGD Processes. Paper presented at the SPE Heavy Oil Conference, Alberta, 12-14 June. SPE-146996-MS.

Aziz, K. and Settari, A. 1979. Petroleum Reservoir Simulation, Applied Science Publishers. Bancroft, W.D. 1913. The Theory of Emulsification V. Journal of Physical Chemistry 17: 501519.

Brice, B. W., Ning, S., Wood, A., and Renouf, G. 2014. Optimum Voidage Replacement Ratio and Operational Practice for Heavy Oil Waterfloods. Paper presented at the SPE Heavy Oil Conference, Calgary, Alberta, 10-12 June. SPE-170099-MS.

Brooks, R.H. and Corey, A.T. 1964. Hydraulic Properties of Porous Media. Hydrology Papers, No. 3, Colorado State U., Fort Collins, Colorado.

Brice, B.W. and Renouf, G. 2008. Increasing Oil Recovery from Heavy Oil Waterfloods. Paper presented at the International Thermal Operations and Heavy Oil Symposium, Calgary, Alberta, 20-23 October. SPE-117327-MS.

Buckley, S.E. and Leverett, M.C. 1942. Mechanism of Fluid Displacements in Sands. AIME 146, 1007-116.

Butler, R. 1997. The Steam and Gas Push (SAGP). Annual Technical Meeting, Petroleum Society of Canada, Calgary, Alberta.

Butler, R.M. 1985. A New Approach to the Modelling of Steam Assisted Gravity Drainage. Journal of Canadian Petroleum Technology 24 (3): 42-51.

Butler, R.M. and Mokrys, I.J. 1989. Solvent Analog Model of Steam-Assisted Gravity Drainage. AOSTRA Journal of Research 5 (1): 17-32. 
Butler, R.M. and Mokrys, I.J. 1990. A New Process (VAPEX) for Recovering Heavy Oils Using Hot Water and Hydrocarbon Vapour. Journal of Canadian Petroleum Technology 29 (6).

Butler, R.M., Jiang, Q. and Yee, C.T. 2000. Steam and Gas Push (SAGP)-3; Recent Theoretical Developments and Laboratory Results. (08).

Canadian Heavy Oil Association. No date. CHOPS Heavy Oil 101. www.choa.ab.ca. Date accessed: 15 November 2017.

Castellanos-Diaz, O., Verlaan, M.L. and Hedden, R. 2016, March. Solvent Enhanced Steam Drive: Results from the First Field Pilot in Canada. In SPE EOR Conference at Oil and Gas West Asia. Society of Petroleum Engineers.

Chen, S., Seib, B., Ben-Zvi, A., and Robinson, T. 2018. Christina Lake Early Rise Rate Solvent Aided Process Pilot. Paper presented at SPE Canada Heavy Oil Technical Conference, Calgary, 13-14 March, 2018, SPE-189756-MS.

Chu, C., 1985. State-Of-The-Art Review of Steamflood Field Projects. Journal of Petroleum Technology 37 (1): 1887-1902.

CMG STARS User Guide Advanced Processes \& Thermal Reservoir Simulator, version 2016.

CMG WinProp User Guide Phase Behaviour and Fluid Property Program, Version 2016.

ConocoPhillips, 2009. Challenged Resources.

Corey, A.T. 1954. The Interrelation Between Gas and Oil Relative Permeabilities. Producers Monthly 19 (November): 38-41.

Corey, A.T. and Rathjens, C.H. 1956. Effect of Stratification on Relative Permeability. Journal of Petroleum Technology 8 (12): 69-71. 
Dembicki, E. 2007. An Overview of Alberta Oil Sands and Recovery Technologies. Presentation at the XVIII Latin American Petroleum Show, Maracaibo, Venezuala, 11-14 June.

Dennis, J.E. Jr. and Schnabel, R.B. 1983. Numerical Methods for Unconstrained Optimization and Nonlinear Equations. Prentice-Hall Series in Computation Math, Cleve Moler, Advisor, 1983.

Diedro, F., Bryan, J., Kryuchkov, S., and Kantzas, A. 2015. Evaluation of Diffusion of Light Hydrocarbon Solvents in Bitumen. Paper presented at SPE Canada Heavy Oil Conference, Calgary, Alberta, 9-11 June. SPE-174424-MS.

Downey, J. No date. Understanding SAGD. http://jeromedowney.ca/blog/understanding-sagd. Date accessed: 25 November 2017.

Energy Resources Conservation Board [ERCB], 2009a.

Energy Solutions Overview by Travis Berrier.

Ezeuko, C.C., Wang, J., and Gates, I.D. 2012. Investigation of Emulsion Flow in SAGD and ESSAGD. Paper presented at the SPE Heavy Oil Conference, Calgary, Alberta, 12-14 June. SPE157830-MS.

Ferguson, M.A., Mamora, D.D., and Goite, J.G. 2001. Steam-Propane Injection for Production Enhancement of Heavy Morichal Oil. Paper SPE 69689 presented at the SPE International Thermal Operations and Heavy Oil Symposium, 12-14 March.

Freitag, N.P. 2008. Edam PVT Study, JIVE Project, PTRC Report, March 2008.

Freitag, N.P. 2015. Heavy Oil Viscosity Dependence on Volatile Solvent Concentration: Discovery of a Universal Behaviour. Paper WHOC15-368 presented at the World Heavy Oil Congress, Edmonton, Alberta, Canada, 24-26 March. 
Gates, I.D. and Chakrabarty, N. 2008. Design of the Steam and Solvent Injection Strategy in Expanding Solvent Steam-Assisted Gravity Drainage. Journal of Canadian Petroleum Technology 47 (9): 12-20.

Gokhan, C., Khosrow, N., and Tayfun, B. 2013. An Enhanced Oil Recovery Technology as a Follow Up to Cold Heavy Oil Production with Sand. SPE-165385-MS. Paper presented at SPE Heavy Oil Conference-Calgary, Alberta, 11-13 June.

Gupta, S. and Gittins, S. 2006. Christina Lake Solvent Aided Process. Journal Canadian Petroleum Technology 45 (9): 49-53.

Gupta, S., Gittins, S., and Picherack, P. 2003. Insights into Some Key Issues with Solvent Aided Process. Journal of Canadian Petroleum Technology 43 (2): 54-61.

Gupta, S., Gittins, S., and Picherack, P. 2005. Field Implementation of Solvent Aided Processes. Journal of Canadian Petroleum Technology 44 (11): 8-13.

Gupta, S., Gittins, S., and Picherack, P., 2005. Field Implementation of Solvent Aided Process. Journal of Canadian Petroleum Technology 44 (11).

Gupta, S., Gittins, S., Sood, A., and Zeidani, K. 2010. Optimal Amount of Solvent in Solvent Aided Process. Paper CSUG/SPE 137543 presented at the Canadian Unconventional Resources \& International Petroleum Conference, Calgary, Alberta, 19-21 October.

Gupta, S.C. and Gittins, S.D. 2006. Christina Lake Solvent Aided Process Pilot. Journal of Canadian Petroleum Technology 45 (09).

Harding, T., Zanon, S., Imran, M. and Kerr, R. 2016. In-Situ Reflux: An Improved In-Situ Recovery Method for Oil Sands. Paper presented at the SPE Heavy Oil Technical Conference, Calgary, 7-9 June. SPE-180752-MS. 
Hossein., E. R., and Anjani. K. 2015. Important Mechanisms and Their Impact on Field Scale Simulation Model. Paper presented at SPE Canada Technical Conference held in Calgary, Alberta, 9-11 June. SPE-174494-MS.

http://webbook.nist.gov/chemistry/fluid/. Date accessed: 15 November 2017.

http://www.chemicalflooding.com. Date accessed: 20 November 2017.

http://www.energy.alberta.ca/OilSands/791.asp. Date accessed: 15 November 2017.

http://www.steamflooding.com, Date accessed 10 December 2017.

https://www.capp.ca/canadian-oil-and-natural-gas/oil/oil-development. Date accessed: 01 November 2017.

Image (C) MKD, based on "Pharmaceutics: The Science of Dosage Form Design" 2nd edition, Michael E. Aulton. Churchill Livingstone, 2002. p. 96.

Imperial Oil. No date. Operations: Oil Sands. :http://www.imperialoil.ca/enca/company/operations/oil-sands/cold-lake. Date accessed 10 December 2017.

Ito, Y., Ichikawa, M., and Hirata, T. 2001. The Effect of Gas Injection on Oil Recovery during SAGD Projects. Journal of Canadian Petroleum Technology 40 (01).

Ivory, J., Frauenfeld, T., and Jossy, C. 2007. Thermal Solvent Reflux and Thermal Solvent Hybrid Experiments. Paper 2007-067 presented at the Petroleum Society's $8^{\text {th }}$ Canadian International Petroleum Conference (58 ${ }^{\text {th }}$ Annual Technical Meeting), Calgary, Alberta, Canada, 12 - 14 June.

Jiang. H., Deng, X., Huang, H., Beaulieu, G., and Heck, G. 2012. Study of Solvent Injection Strategy in ES-SAGD Process. Paper presented at the SPE Heavy Oil Conference, Calgary, Alberta. 12-14 June 2012. SPE-157838-MS. 
Khaledi, R., Bechman, M., Pustanky. K., Mohan A., Watterbarger. C., and Dickson. J. 2012. Physical Modelling of SA-SAGD. Paper presented at the SPE Heavy Oil Conference, Calgary, Alberta, 12-14 June. SPE-150676-MS.

Khaledi, R., Boone, T. J., Motahhari, H. R., and Subramanian, G. 2015, 9 June. Optimized Solvent for Solvent Assisted-Steam Assisted Gravity Drainage (SA-SAGD) Recovery Process. Society of Petroleum Engineers. doi:10.2118/174429-MS.

King, R. W., Zhao, L., Huang, H., McFarlane, R.A., and Gao, J. 2005. Steam and Solvent Coinjection Experiment: Fluid Property Characterization. Paper SPE/PS-CIM/CHOA 97842 presented at the 2005 SPE International Thermal Operations and Heavy Oil Symposium, Calgary, Alberta, Canada, 1-3 November.

Knorr, K.D. 2014. Cold Heavy Oil Production with Sand. Presented at the World Heavy Oil Congress, Calgary.

Knorr, K.D. and Imran, M. 2011. Extension of Das and Butler Semianalytical Flow Model. Journal of Canadian Petroleum Technology 50 (6): 1-9.

Knorr, K.D. and Imran, M. 2012. Solvent Chamber Development in 3D Physical Model Experiments of SVX Process with Various Permeabilities and Solvent Vapour Qualities. Journal of Canadian Petroleum Technology 51 (6). SPE-149190-PA.

Leaute, R.P. 2002. Liquid Addition to Steam for Enhancing Recovery (LASER) of Bitumen with CSS: Evolution of Technology from Research Concept to a Field Pilot at Cold Lake. In SPE International Thermal Operations and Heavy Oil Symposium and International Horizontal Well Technology Conference. Society of Petroleum Engineers.

Li, Y., Liu, S., and Liu, C. 2012. Impact of Non Condensable Gas on SAGD Performance. SPE 150539 presented at SPE Heavy oil Conference, Alberta, 12-14 June. 
Mehrotra, A. K. and Svrcek, W. Y. 1982. Correlations for properties of bitumen saturated with $\mathrm{CO} 2, \mathrm{CH} 4$ and $\mathrm{N} 2$, and experiments with combustion gas mixtures. Journal of Canadian Petroleum Technology November-December 1982, Montreal.

Mohiddin, J.G. and Koci, P.F. 2007. Peace River Carmon Creek Project - Optimization of Cyclic Steam Stimulation through Experimental Design. Paper presented at the Annual Technical Conference and Exhibition, Anaheim, California, 11-14 November. SPE-109826-MS.

Nasr, T.N. and Ayodele, O.R. 2005. Thermal Techniques for the Recovery of Heavy Oil and Bitumen. Paper SPE97488-MS-P presented at SPE International Improved Oil Recovery Conference in Asis Pacific, Kuala Lumpur, Malaysia, 5-6 December.

Nasr, T.N. and Ayodele, O.R. 2006. New Hybrid Steam-Solvent Processes for the Recovery of Heavy Oil and Bitumen.. Paper 101717 presented at SPE International Petroleum Exhibition and Conference, Abu-Dhabi, U.A.E, 5-8 November.

Nasr, T.N., Beaulieu, G., Golbeck, H., and Heck , G. 2003. Novel Expanding Solvent-SAGD Process “ES-SAGD”. Journal of Canadian Petroleum Technology 42 (1): 13-16.

NETL. 2010. Carbon Dioxide Enhanced Oil Recovery: Untapped Domestic Energy Supply and Long Term Carbon Storage Solution. U.S. Department of Energy. Available at www.netl.doe.gov/technologies/oil-gas/publications/EP/carbon dioxide_EOR_Primer.pdf. Date accessed: 20 Nov 2017.

Noik, C., Dalmazzone, C., Goulay, C., and Glenat, P. 2005. Characterization and Emulsion Behaviour of Athabasca Extra Heavy Oil produced by SAGD. Paper presented at SPE/PSCIM/CHOA International Thermal Operations and Heavy Oil Symposium, Calgary, Alberta, 1-3 November. 
Oballa, V., Coombe, D.A., and Buchanan, W.L. 1989. Adaptive Implicit Method in Thermal Simulation. Paper SPE 18767 presented at the 1989 SPE California Regional Meeting, Bakersfield, California, 5-7 April.

Pedersen, K.S. and Fredenslund, A. 1987. An Improved Corresponding States Model for the Prediction of Oil and Gas Viscosities and Thermal Conductivities. Chemical Engineering Science 42 (1): 182-186.

Peng, D. Y. and Robinson, D. B. (1976). A New Two-Constant Equation of State. Industrial and Engineering Chemistry: Fundamentals 15: 59-64.

Pujol, L. and Boberg, T.C. 1972. Scaling Accuracy of Laboratory Steam Flooding Models. Paper SPE 4191 presented at the California Regional Meeting, Bakersfield, California, 8-10 November.

Simangunsong, R., Jaiswal, N.J., and Mamora, D.D. 2006. Improved Analytical Model and Experimentally Calibrated Studies of Steam Injection With Hydrocarbon Additives to Enhance Heavy-Oil Recovery. Paper SPE 100703 presented at the 2006 SPE Annual Technical Conference and Exhibition, San Antonio, Texas, 24-27 September.

Stark, S.D. 2013, March. Cold Lake Commercialization of the Liquid Addition to Steam for Enhancing Recovery (LASER) Process. In IPTC 2013: International Petroleum Technology Conference.

Taupy, J-A. 2007. DME a Clean Fuel for Future. Paper presented at the SPE International Technology Conference, Dubai, UAE, 4-6 December. SPE IPTC 11754

Tremblay, B. 2005. Modelling of Sand Transport Through Wormholes. Journal of Canadian Petroleum Technology 44 (4).

US Energy Information Administration. 2007. World Proved Reserves of Oil and Natural Gas. Retrieved 2008-08-19.

Winter Pilot Expansion SPRI Final Report, SPRI Project Agreement Number 7405-023. 
Zeidani, K. and Gupta, S.C. 2013. Surfactant-Steam Process: An Innovative Enhanced Heavy Oil Recovery Method for Thermal Applications. Paper presented at the SPE Heavy Oil ConferenceCanada, Calgary, 11-13 June. SPE 165545-MS. https://doi.org/10.2118/165545-MS.

Zhao, B., Becerra, M., and Shaw, J.M. 2009. On Asphaltene and Resin Association in Athabasca Bitumen and Maya Crude Oil. Energy Fuels 23: 4431-4437. 